\title{
Location and Description of Transects for Ecological Studies in Floodplain Forests of the Lower Suwannee River, Florida
}

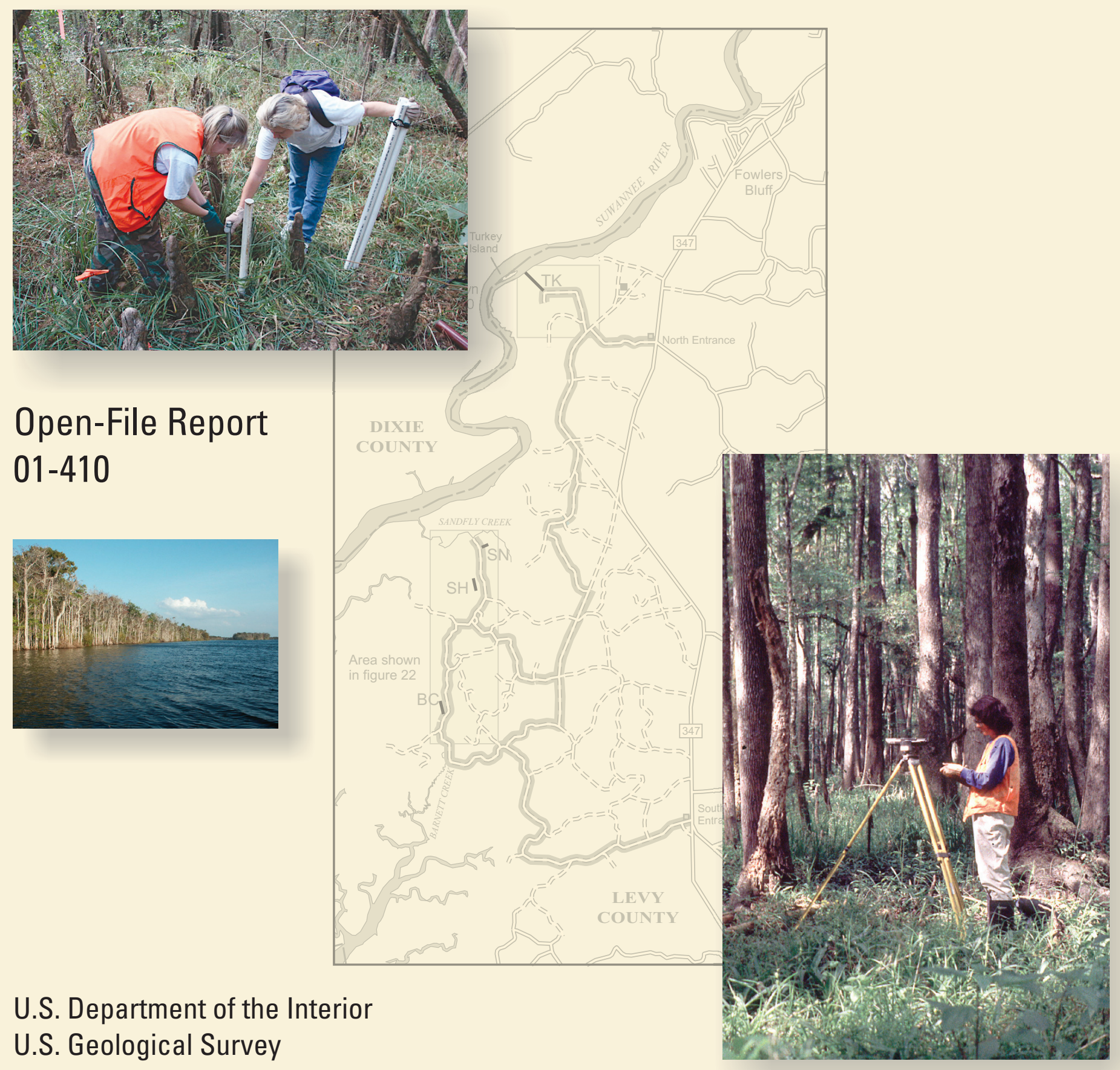

Prepared in cooperation with the

Suwannee River Water Management District 


\section{Location and Description of Transects for Ecological Studies in Floodplain Forests of the Lower Suwannee River, Florida}

By Lori J. Lewis, Helen M. Light, and Melanie R. Darst

U.S. GEOLOGICAL SURVEY

Open File Report 01-410

Prepared in cooperation with the

Suwannee River Water Management District

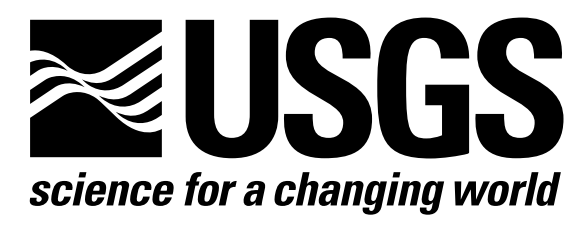

Tallahassee, Florida 


\title{
U.S. DEPARTMENT OF THE INTERIOR \\ GALE A. NORTON, Secretary
}

\author{
U.S. GEOLOGICAL SURVEY
}

CHARLES G. GROAT, Director

The use of firm, trade, and brand names in this report is for identification purposes only and does not constitute endorsement by the U.S. Geological Survey.

For additional information write to:

\section{District Chief}

U.S. Geological Survey

Suite 3015

227 N. Bronough Street

Tallahassee, FL 32301
Copies of this report can be purchased from:

U.S. Geological Survey Branch of Information Services Box 25286

Denver, CO 80225-0286 888-ASK-USGS

Additional information about water resources in Florida is available on the Internet at http://fl.water.usgs.gov 


\section{CONTENTS}

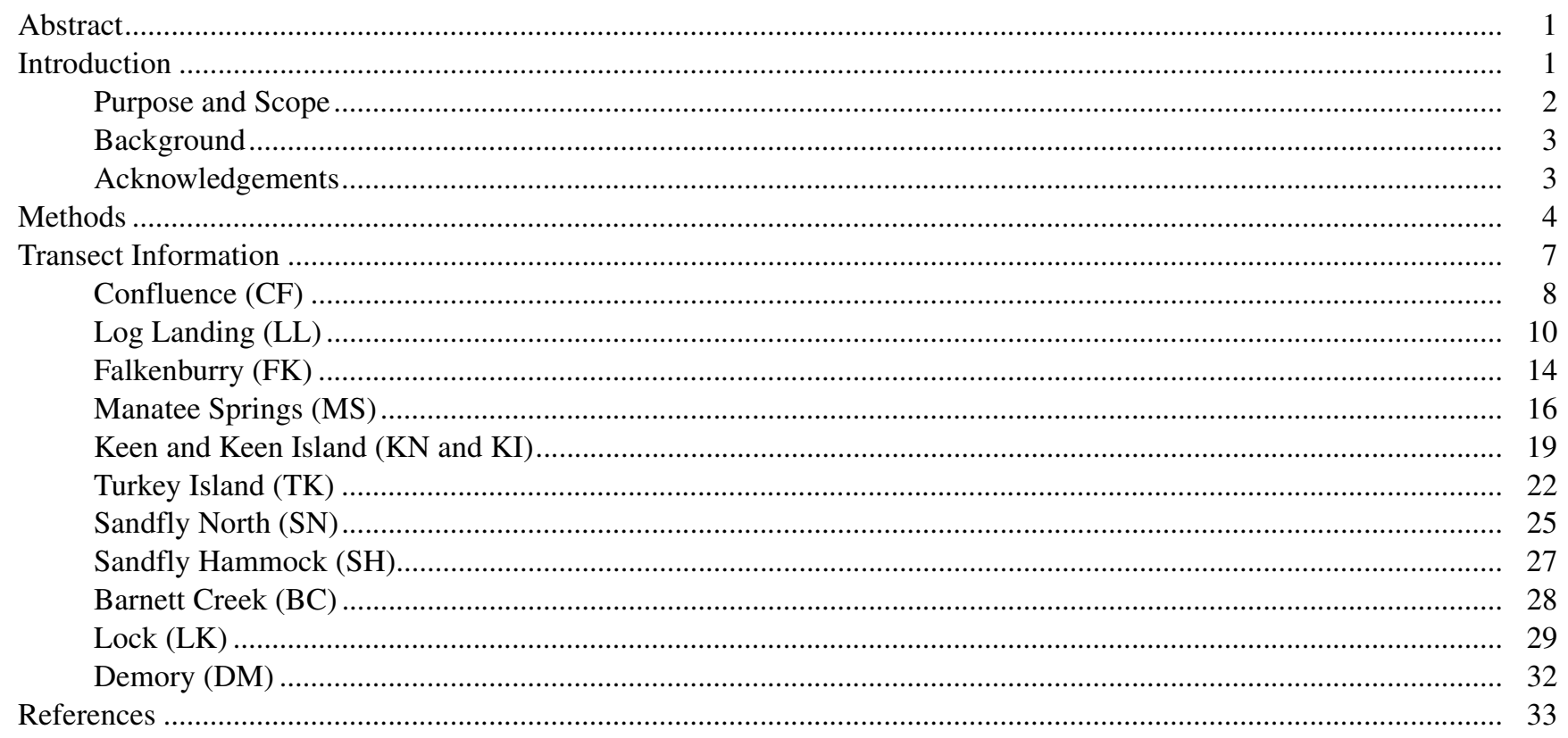

\section{APPENDIXES:}

Appendix 1. Canopy tree species and measurements and marker locations on transects in the lower Suwannee River floodplain, Florida

Appendix 2. Conversion tables for determining the permanent marker numbers of all previous stake numbers recorded in original U.S. Geological Survey field notes....

\section{FIGURES}

\section{1-2. Maps showing:}

1. Drainage basin of the Suwannee River in Florida and Georgia .................................................... 2

2. Study area with locations of transects in the floodplain of the lower Suwannee River, Florida................... 5

3-4. Photographs showing:

3. Permanent marker with PVC sleeve used to mark transects in the lower Suwannee River floodplain, Florida.

4. PVC pipe installed adjacent to marker used to mark transects in the lower Suwannee River floodplain, Florida.

5-6. Maps showing:

5. CF transect and surrounding area in the lower Suwannee River floodplain, Florida. ................................... 9

6. CF transect with marker locations and access roads in the lower Suwannee River floodplain, Florida......... 9

7. Graph showing land-surface elevations and marker locations at CF transect in the lower Suwannee River floodplain, Florida.

8-9. Maps showing:

8. LL and FK transect locations with surrounding area in the lower Suwannee River floodplain, Florida........ 11

9. LL transect with marker locations and immediate vicinity in the lower Suwannee River floodplain, Florida. 12

10. Graph showing land-surface elevations and marker locations at LL transect in the lower Suwannee River floodplain, Florida. 
11. Map showing FK transect with marker locations and access roads in the lower Suwannee River floodplain, Florida.

12. Graph showing land-surface elevations and marker locations at FK transect in the lower Suwannee River floodplain, Florida.

13-14. Maps showing

13. MS, KN and KI transect locations and surrounding area in the lower Suwannee River floodplain, Florida. 17

14. MS transect with marker locations and access roads in the lower Suwannee River floodplain, Florida. ...... 18

15. Graph showing land-surface elevations and marker locations at MS transect in the lower Suwannee River floodplain, Florida.

16. Map showing KN and KI transects with marker locations and access roads in the lower Suwannee River floodplain, Florida.

17-18. Graphs showing:

17. Land-surface elevations and marker locations at KN transect in the lower Suwannee River Floodplain, Florida.

18. Land-surface elevations and marker locations at KI transect in the lower Suwannee River Floodplain, Florida.

19-20. Maps showing:

19. TK, SN, SH, and BC transect locations with surrounding area in the lower Suwannee River floodplain, Florida

20. TK transect with marker locations and access roads in the lower Suwannee River floodplain, Florida........ 23

21. Graph showing land-surface elevations and marker locations at TK transect in the lower Suwannee River floodplain, Florida.

22. Map showing SN, SH, and BC transects with marker locations and access roads in the lower Suwannee River floodplain, Florida.

23-25. Graphs showing:

23. Land-surface elevations and marker locations at SN transect in the lower Suwannee River floodplain, Florida......

24. Land-surface elevations and marker locations at SH transect in the lower Suwannee River floodplain, Florida

25. Land-surface elevations and marker locations at BC transect in the lower Suwannee River floodplain, Florida

26-27. Maps showing:

26. LK and DM transect locations and surrounding area in the lower Suwannee River floodplain, Florida.......

27. LK and DM transects with marker locations and access roads in the lower Suwannee River floodplain, Florida.

28-29. Graphs showing:

28. Land-surface elevations and marker locations at LK transect in the lower Suwannee River floodplain, Florida.

29. Land-surface elevations and marker locations at DM transect in the lower Suwannee River floodplain, Florida 
TABLES

1. Location and marker information of transects in the lower Suwannee River floodplain, Florida....................... 7

2. Marker locations on CF transect in the lower Suwannee River floodplain, Florida......................................... 8

3. Marker locations on LL transect in the lower Suwannee River floodplain, Florida..........................................13

4. Marker locations on FK transect in the lower Suwannee River floodplain, Florida ....................................... 15

5. Marker locations on MS transect in the lower Suwannee River floodplain, Florida.......................................... 16

6. Marker locations on KN transect in the lower Suwannee River floodplain, Florida..................................... 21

7. Marker locations on KI transect in the lower Suwannee River floodplain, Florida ....................................... 21

8. Marker locations on TK transect in the lower Suwannee River floodplain, Florida ...................................... 24

9. Marker locations on SN transect in the lower Suwannee River floodplain, Florida ...................................... 26

10. Marker locations on SH transect in the lower Suwannee River floodplain, Florida ........................................27

11. Marker locations on BC transect in lower Suwannee River Floodplain, Florida ........................................... 28

12. Marker locations on LK transect in the lower Suwannee River floodplain, Florida ......................................... 31

13. Marker locations on DM transect in the lower Suwannee River floodplain, Florida ....................................... 32

CONVERSION FACTORS, SEA LEVEL DATA, AND ACRONYMS

\begin{tabular}{rll}
\hline Multiply & By & To obtain \\
\hline centimeter $(\mathrm{cm})$ & 0.3937 & inch \\
meter $(\mathrm{m})$ & 3.28 & foot \\
kilometer $(\mathrm{km})$ & 0.62 & mile \\
\hline
\end{tabular}

Sea level: In this report, "sea level" refers to the National Geodetic Vertical Datum of 1929 (NGVD of 1929) — a geodetic datum derived from a general adjustment of the first- order level nets of the United States and Canada, formerly called Sea Level Datum of 1929.

Horizontal datum: In this report, horizontal coordinate information is referenced to the North American Datum of 1927 (NAD27).

\section{ACRONYMS}

$\begin{aligned} \text { GIS } & =\text { Geographic Information Systems } \\ \text { LSNWR } & =\text { Lower Suwannee National Wildlife Refuge } \\ \text { GPS } & =\text { Global Positioning System } \\ \text { rkm } & =\text { river kilometer } \\ \text { SRWMD } & =\text { Suwannee River Water Management District } \\ \text { USGS } & =\text { U.S. Geological Survey }\end{aligned}$

\section{CONTACT INFORMATION}

\begin{tabular}{ll}
\hline Florida Department of Environmental Protection, & \\
$\quad$ Division of Recreation and Parks & (352) $955-2135$ \\
Lower Suwannee National Wildlife Refuge & (352) $493-0238$ \\
Manatee Springs State Park & (352) $493-6738$ \\
Suwannee River Water Management District & (800) $226-1066$ \\
\hline
\end{tabular}


VI Contents 


\title{
Location and Description of Transects for Ecological Studies in Floodplain Forests of the Lower Suwannee River, Florida
}

\author{
By Lori J. Lewis, Helen M. Light and Melanie R. Darst
}

\begin{abstract}
Twelve transects were established in floodplain forests along the lower Suwannee River, Florida, as the principal data collection sites for a comprehensive study conducted by the U.S. Geological Survey and the Suwannee River Water Management District from 1996 to 2001. Data collected along the 12 transects included hydrologic conditions, land-surface elevations, soils, and vegetation of floodplain forests in relation to river flow. Transect locations are marked in the field with permanent markers at approximately 30 meter intervals.

Detailed descriptions of the 12 transects and their locations are provided so that they can be used for future ecological studies. Descriptions of the transects include contact information necessary for access to the property on which the transects are located, maps showing transect locations and routes from the nearest city or major road, small scale maps of each transect showing marker locations, latitude and longitude of each marker, compass bearings of each transect line and graphs showing land-surface elevations of the transect with marker locations.
\end{abstract}

\section{INTRODUCTION}

This report is part of a comprehensive study of floodplain forests along the lower Suwannee River, Florida, conducted by the U.S. Geological Survey (USGS) in cooperation with the Suwannee River Water Management District (SRWMD) from 1996 to 2001. The purpose of the study was to help determine water needs for maintaining healthy wetland ecosystems in the lower Suwannee River basin to assist the SRWMD in the establishment of minimum flows and levels for the river.

Data collected along twelve study transects included hydrologic conditions, land-surface elevations, soils, and vegetation of floodplain forests in relation to river flow (Light and others, in press; Darst and others, 2002). Locations and descriptions of the transects are presented to enable others to use these transects for future wetland studies such as longterm monitoring, land management studies, or interdisciplinary studies that could build on the information that has already been collected. 
Purpose and Scope

The objective of this report is to document the location and description of twelve permanently marked transects in the forested floodplain of the lower Suwannee River to allow recovery of these transects for future studies. The study area for this project was the forested floodplain of the Suwannee River from the confluence at the Santa Fe River to the downstream limit of forests near the Gulf of Mexico (fig. 1).

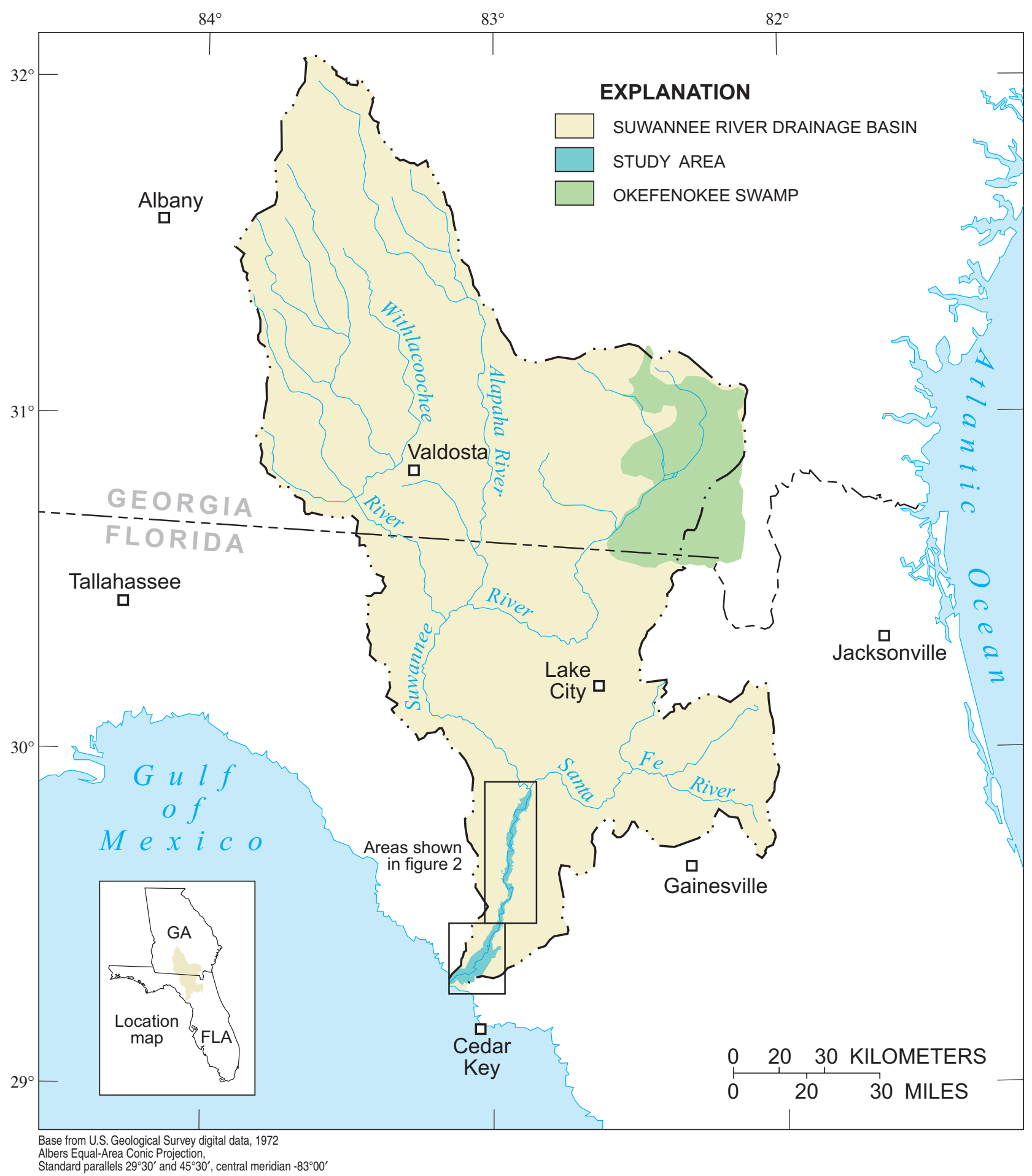

Figure 1. Drainage basin of the Suwannee River in Florida and Georgia. 


\section{Background}

The principal sites of data collection for a comprehensive wetlands study were 12 belt transects established in the lower Suwannee River floodplain (fig. 2). A belt transect is a long, narrow rectangular sampling area oriented along a centerline with a width of a few meters on one or both sides of the line.

In the comprehensive wetland study, 14 forest types were defined from vegetative sampling and aerial photographic signatures at the transects. Extensive vegetative, topographic, hydrologic and soils data collected on the transects were described and analyzed by forest type in a report by Light and others (in press). Ground cover vegetation data was described by forest type in Darst and others, 2002. GIS coverages of a forest map and the transect locations can be obtained from SRWMD or USGS files.

Specific transect data that is available for use by future researchers is briefly described in the following paragraphs. In the remainder of this background section, all appendix, table, and figure numbers refer to data presented in the comprehensive report by Light and others (in press).

The specific location of the forest types at each transect are illustrated along with land-surface elevations of the transects and longterm hydrologic conditions in the Suwannee River in figure 15. The median elevations of the forest types at each transect (app. II) were compared to daily high stage at each transect in the tidal portion of the Suwannee River in figure 16.

Water level measurements were made in the river and in floodplain water bodies at the transects (app. III). Salinity of ponds and tidal creeks at selected lower tidal transects is described in appendix IV and figure 20.

Soil profiles were described at each transect at boring locations selected to represent various topographic features and tree communities (table 9, fig. 22 and app. V). Soil moisture observations were made at regular intervals along each transect line (app III). The amount of continuously saturated soils for the forest types at each transect is reported in table 10. Soil conductivity measured at transects in the lower tidal portion of the river was reported in table 11 and figure 23.

\section{Acknowledgements}

The authors are grateful to USGS employees Darlene Blum, Warren Carmichael, Gary Mahon, Rich Marella, Terry Petrosky, Agustin Sepulveda, and Jim Tomberlin for their help in the installation of 170 markers in the floodplain.

The authors would like to thank John Good and Rob Mattson, SRWMD for technical reviews, and the following USGS employees: Jerilyn Collins, Gary Mahon, Jane Eggleston and Teresa Embry for technical and editorial reviews; Agustin Sepulveda for geographic information systems (GIS) assistance; Ron Spencer and Jim Tomberlin for assistance with graphics; and Pat Mixson for layout.

Access to study sites was received from Ken Litzenberger, Lower Suwannee National Wildlife Refuge (LSNWR); Susie Hetrick, Florida Department of Parks and Recreation; Sally Lieb, Manatee Springs State Park; and Stephen Riggs, private landowner. 


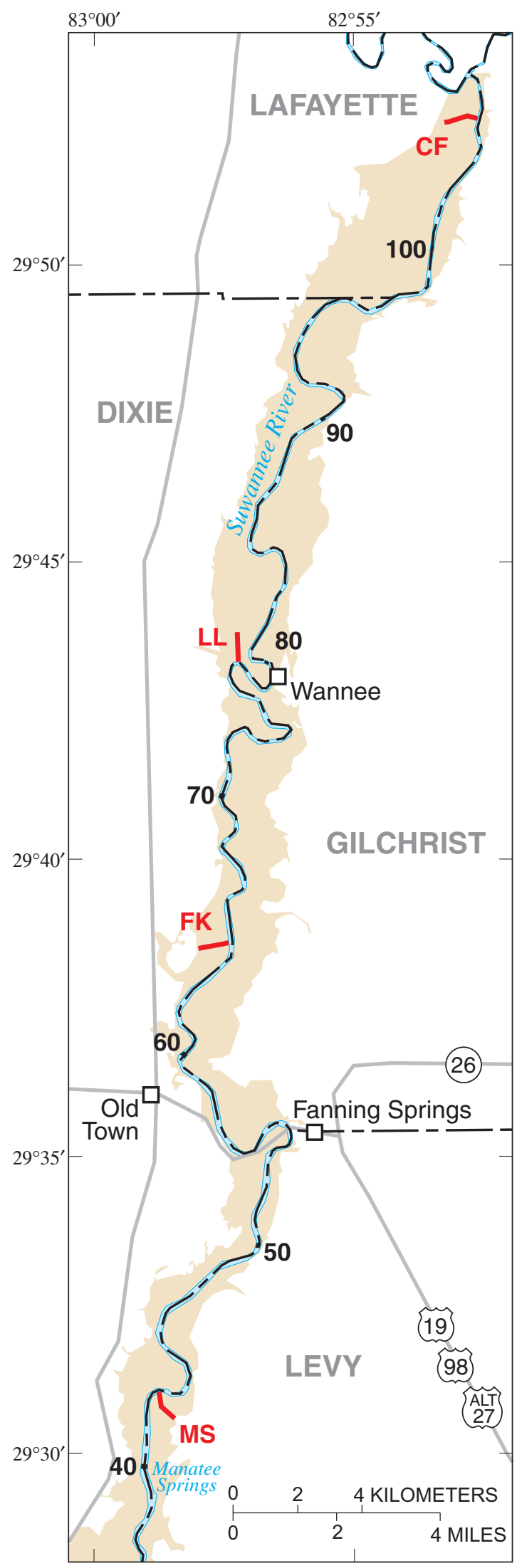

Base from U.S. Geological Survey digital data, 1972 Albers Equal-Area Conic Projection

Standard parallels $29^{\circ} 30^{\prime}$ and $45^{\circ} 30^{\prime}$, central meridian $-83^{\circ} 00^{\prime}$

\section{METHODS}

Data collection efforts in the comprehensive study began in 1996 with the marking of transect lines with wooden stakes at approximately 30 meter intervals. The stakes had begun to show signs of deterioration by the end of the study period. The sites were revisited in 2001 and the wooden stakes replaced with 1/2-inch galvanized pipes labeled with aluminum tags for identification. They were installed so that approximately 18 inches of pipe was visible above the ground. For additional visibility, PVC pipes were installed around the galvanized pipe as a sleeve (fig. 3), or next to the galvanized pipe when conditions prevented sleeve-type installation (fig. 4).

Stainless steel rods (9/16-inch diameter) were installed at each transect endpoint to serve as backup reference points in case the visible permanent markers are damaged or destroyed by flood, fire, or vandalism. These rods were four feet in length and were driven to ground surface level so their location would be recoverable by use of a metal detector. Once the transect endpoint is found, the other transect marker locations can be reconstructed using compass bearings and measured distances, with latitude and longitude of marker locations as an additional guide.

The map position of each transect marker was obtained from the GIS coverage of the transects. Latitudes and longitudes were determined using an ARC macro language program that converts map units to geographic locations.

\section{EXPLANATION}

STUDY AREA

FK - TRANSECT

60 DISTANCE FROM RIVER MOUTH-In kilometers

Figure 2. Study area with locations of transects in the floodplain of the lower Suwannee River, Florida. 


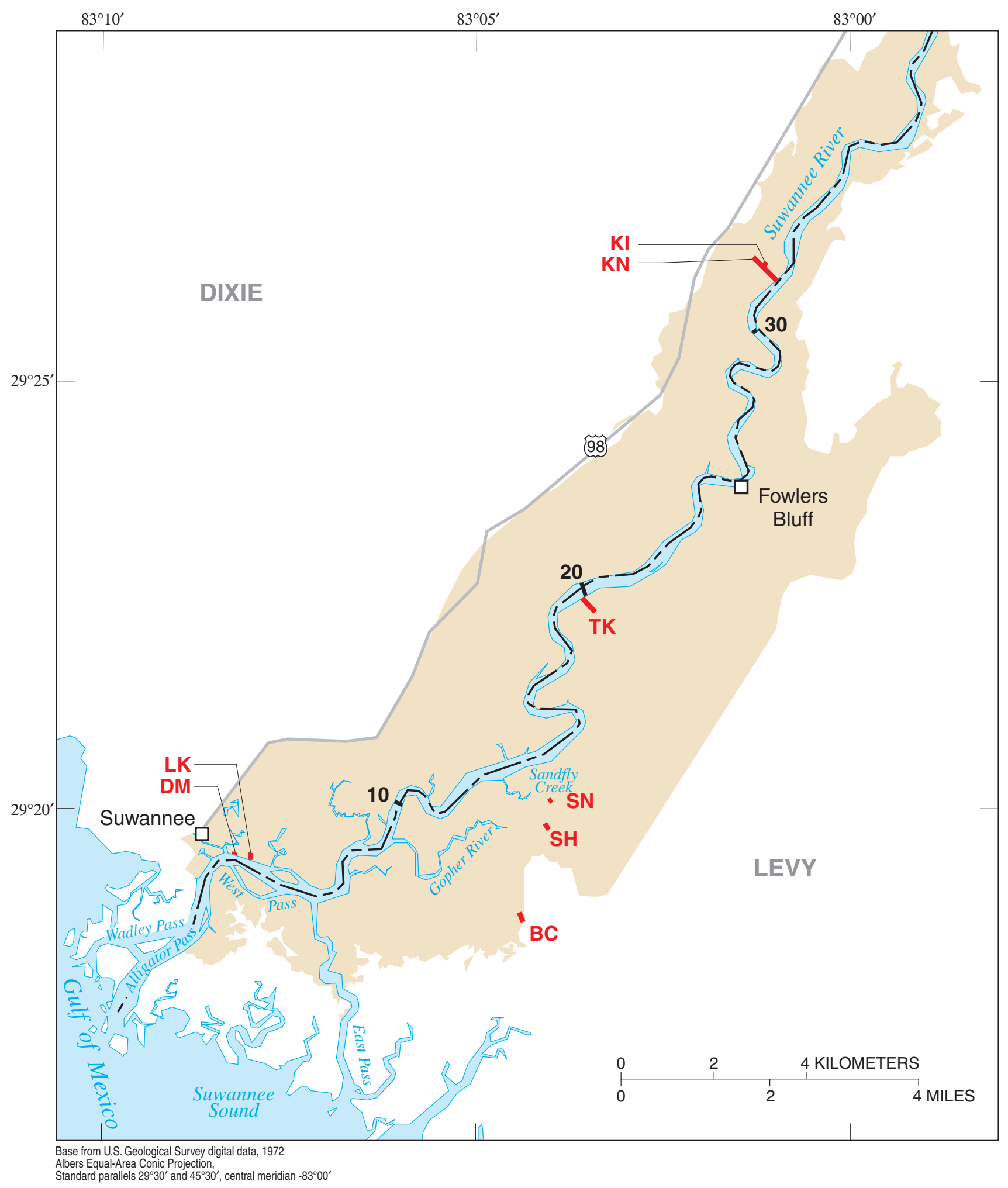

Figure 2. Study area with locations of transects in the floodplain of the lower Suwannee River, Florida. (Continued) 


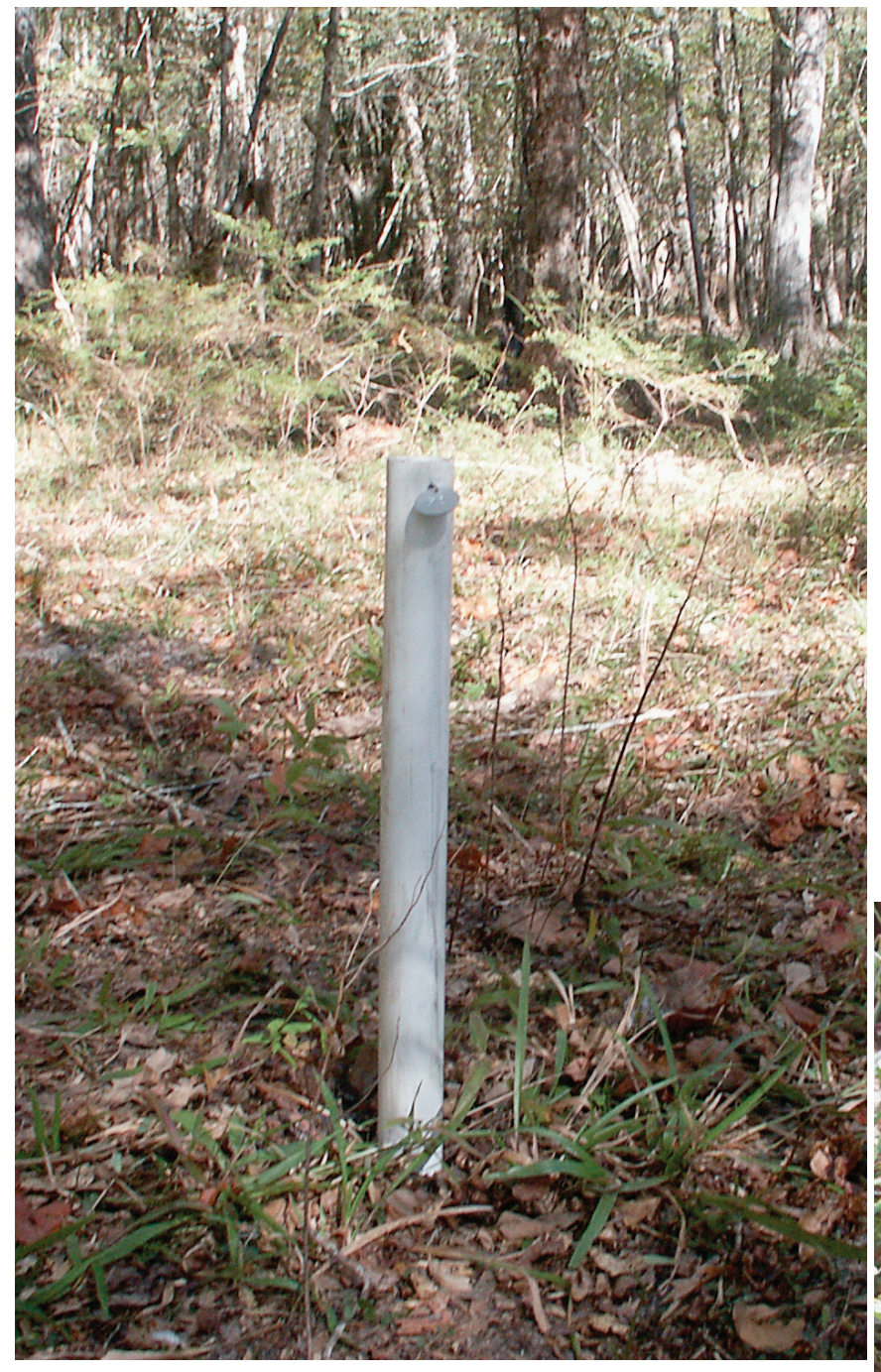

Figure 4. PVC pipe installed adjacent to marker used to mark transects in the lower Suwannee River floodplain, Florida.
Figure 3. Permanent marker with PVC sleeve used to mark transects in the lower Suwannee River floodplain, Florida.

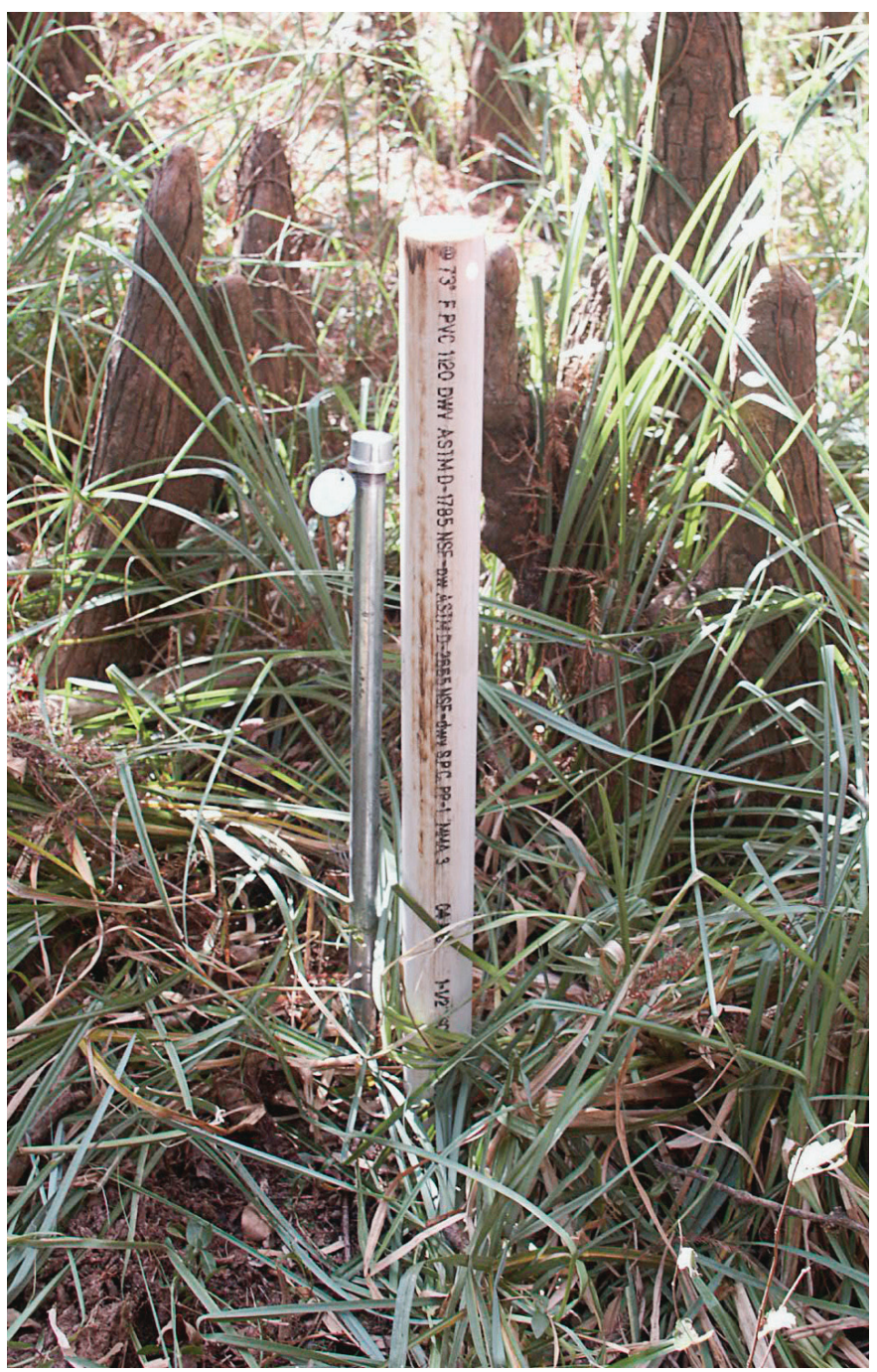


TRANSECT INFORMATION

Descriptions of the transects are presented in downstream order. Directions by road are described using distances in miles; all other units in this report are metric. Basic data for the transects are included in table 1. Transect locations along the river are indicated in river kilometers (rkm), a measurement of stream distance beginning with 0 at the mouth of the river at latitude $29^{\circ} 17^{\prime} 19.2^{\prime \prime}$ and longitude $83^{\circ} 9^{\prime} 51.8^{\prime \prime}$.

Transects were named after the tract of land on which they were located or after a nearby creek or point of interest. Transects varied in length from 53.2 to $1,003.7 \mathrm{~m}$ and were 10 to $13 \mathrm{~m}$ wide when less than $400 \mathrm{~m}$ long and usually $5 \mathrm{~m}$ wide when longer.

Transects are located primarily on public lands with at least one end of the transect accessible by boat or car. Permission from current land managers is needed to obtain access to these properties. The necessary contact information for property access is provided for each transect.

Maps showing the transect location and route from nearest city or major road are provided. In this report, U.S. and State highways are classified as primary roads, county roads and other paved streets are described as light duty roads, and dirt roads or trails are classified as unimproved roads. GIS coverage maps of each transect showing marker locations and numbers are also provided. Latitude and longitude of each marker are listed in an associated table.

Graphs showing land-surface elevations with marker locations are provided for each transect.
Estimated elevations beyond the transect endpoints or between two segments of a transect are included to show the topographic features of the immediate area. The scales of the elevation graphs have been maximized for each transect for easier viewing of the marker numbers and their locations; therefore, they cannot be used for comparing elevations of different transects. Elevation graphs of all the transects at the same scale are presented in figure 15 of Light and others, in press.

Canopy vegetation data with marker locations is presented for each transect in appendix 1. This information can be used not only as an additional guide for locating markers, but also as baseline data for future vegetative sampling efforts.

Table 1. Location and marker information of transects in the lower Suwannee River floodplain, Florida

[rkm, river kilometers; $\mathrm{m}$, meters]

\begin{tabular}{lcrrrc}
\hline Transect name & $\begin{array}{c}\text { Abbreviated } \\
\text { transect name }\end{array}$ & $\begin{array}{c}\text { Location, } \\
\text { in rkm }\end{array}$ & $\begin{array}{c}\text { Length of } \\
\text { transect, } \\
\text { in m }\end{array}$ & $\begin{array}{c}\text { Number of } \\
\text { markers }\end{array}$ & $\begin{array}{c}\text { Range of } \\
\text { marker } \\
\text { numbers }\end{array}$ \\
\hline Confluence & CF & 104.3 & 441.0 & 21 & $1-22$ \\
Log Landing & LL & 77.6 & 921.2 & 30 & $22-51,200^{1}$ \\
Falkenburry & FK & 64.4 & 362.6 & 15 & $52-66$ \\
Manatee Springs & MS & 42.5 & 1009.0 & 28 & $67-94$ \\
Keen & KN & 31.2 & 734.1 & 27 & $95-121$ \\
Keen Island & KI & 31.2 & 100.0 & 4 & $122-125$ \\
Turkey Island & TK & 19.8 & 411.9 & 15 & $126-140$ \\
Sandfly North & SN & 13.0 & 88.3 & 5 & $141-145$ \\
Sandfly Hammock & SH & 12.6 & 151.0 & 6 & $146-151$ \\
Barnett Creek & BC & 11.3 & 215.6 & 8 & $152-159$ \\
Lock & LK & 5.1 & 145.5 & 6 & $160-165$ \\
Demory & DM & 4.8 & 53.2 & 4 & $166-169$ \\
\hline
\end{tabular}

${ }^{1}$ This marker is not on the transect line; it marks a verification plot near the transect 
CF transect is located on land owned by the SRWMD. A locked gate restricts motor vehicle access to the roads on this property. Contact the SRWMD land manager in Live Oak, Florida at (800) 2261066 for permission to access these lands.

$\mathrm{CF}$ transect is in eastern Lafayette County, approximately 1.6 kilometers downstream of the confluence of the Santa Fe River with the Suwannee River. To reach this transect from Branford, Florida, travel 1.9 miles west on US Highway 27, then 4.3 miles south on State Highway 349 to County Road 138. Go east on County Road
138 and follow this road to the Hatchbend boat ramp using the route highlighted in figure 5 . Turn right on the last dirt road before the boat ramp. Turn left at the T-intersection, travel approximately 1.5 miles and make another left turn. The SRWMD sign and locked gate are less than 0.1 mile down this drive. Once inside the gate, turn left. The roads on this property are very sandy and under certain conditions require the use of a four-wheel drive vehicle. Go approximately $1 / 4$ of a mile, following the $90^{\circ}$ turn to the right, and then look for a smaller trail to the right. The western portion of the transect is acces- sible from this trail. To reach the eastern portion of the transect continue past this trail for another 0.5 mile until the road makes a $90^{\circ}$ turn to the right.

$\mathrm{CF}$ transect is comprised of two segments (fig. 6), the west segment (markers 1-7) and the east segment (markers 8-21). The west segment is $128.3 \mathrm{~m}$ in length and has a compass bearing of approximately $80^{\circ}$ from marker number 1 . The east segment is $312.7 \mathrm{~m}$ long and has a compass bearing of $107^{\circ}$ from marker 8 . The latitude and longitude of each marker on the transect line are listed in table 2.

Table 2. Marker locations on CF transect in the lower Suwannee River floodplain, Florida

\begin{tabular}{|c|c|c|c|c|}
\hline $\begin{array}{l}\text { Marker } \\
\text { number }\end{array}$ & $\begin{array}{l}\text { Distance from } \\
\text { beginning of } \\
\text { segment }\end{array}$ & $\begin{array}{l}\text { Distance from } \\
\text { previous } \\
\text { marker }\end{array}$ & Latitude & Longitude \\
\hline \multicolumn{5}{|c|}{ West Segment } \\
\hline 1 & 0.0 & - & $29^{\circ} 52^{\prime} 24.9^{\prime \prime}$ & $82^{\circ} 53^{\prime} 14.7^{\prime \prime}$ \\
\hline 2 & 20.1 & 20.1 & $29^{\circ} 52^{\prime} 25.0^{\prime \prime}$ & $82^{\circ} 53^{\prime} 14.0^{\prime \prime}$ \\
\hline 3 & 44.6 & 24.5 & $29^{\circ} 52^{\prime} 25.1^{\prime \prime}$ & $82^{\circ} 53^{\prime} 13.1^{\prime \prime}$ \\
\hline 4 & 68.3 & 23.7 & $29^{\circ} 52^{\prime} 25.2^{\prime \prime}$ & $82^{\circ} 53^{\prime} 12.2^{\prime \prime}$ \\
\hline 5 & 88.6 & 20.3 & $29^{\circ} 52^{\prime} 25.3^{\prime \prime}$ & $82^{\circ} 53^{\prime} 11.4^{\prime \prime}$ \\
\hline 6 & 115.5 & 26.9 & $29^{\circ} 52^{\prime} 25.5^{\prime \prime}$ & $82^{\circ} 53^{\prime} 10.4^{\prime \prime}$ \\
\hline 7 & 128.3 & 12.8 & $29^{\circ} 52^{\prime} 25.5^{\prime \prime}$ & $82^{\circ} 53^{\prime} 10.0^{\prime \prime}$ \\
\hline \multicolumn{5}{|c|}{ East Segment } \\
\hline 8 & 0.0 & - & $29^{\circ} 52^{\prime} 31.6^{\prime \prime}$ & $82^{\circ} 52^{\prime} 47.9^{\prime \prime}$ \\
\hline 9 & 22.1 & 22.1 & $29^{\circ} 52^{\prime} 31.4^{\prime \prime}$ & $82^{\circ} 52^{\prime} 47.1^{\prime \prime}$ \\
\hline 10 & 53.9 & 31.8 & $29^{\circ} 52^{\prime} 31.2^{\prime \prime}$ & $82^{\circ} 52^{\prime} 46.0^{\prime \prime}$ \\
\hline 11 & 79.9 & 26.0 & $29^{\circ} 52^{\prime} 31.0^{\prime \prime}$ & $82^{\circ} 52^{\prime} 45.0^{\prime \prime}$ \\
\hline 12 & 106.8 & 26.9 & $29^{\circ} 52^{\prime} 30.7^{\prime \prime}$ & $82^{\circ} 52^{\prime} 44.1^{\prime \prime}$ \\
\hline 13 & 129.4 & 22.6 & $29^{\circ} 52^{\prime} 30.5^{\prime \prime}$ & $82^{\circ} 52^{\prime} 43.3^{\prime \prime}$ \\
\hline 14 & 158.7 & 29.3 & $29^{\circ} 52^{\prime} 30.2^{\prime \prime}$ & $82^{\circ} 52^{\prime} 42.2^{\prime \prime}$ \\
\hline 15 & 187.8 & 29.1 & $29^{\circ} 52^{\prime} 29.9^{\prime \prime}$ & $82^{\circ} 52^{\prime} 41.2^{\prime \prime}$ \\
\hline 16 & 216.3 & 28.5 & $29^{\circ} 52^{\prime} 29.7^{\prime \prime}$ & $82^{\circ} 52^{\prime} 40.2^{\prime \prime}$ \\
\hline 17 & 235.2 & 18.9 & $29^{\circ} 52^{\prime} 29.5^{\prime \prime}$ & $82^{\circ} 52^{\prime} 39.5^{\prime \prime}$ \\
\hline 18 & 256.7 & 21.5 & $29^{\circ} 52^{\prime} 29.3^{\prime \prime}$ & $82^{\circ} 52^{\prime} 38.7^{\prime \prime}$ \\
\hline 19 & 286.1 & 29.4 & $29^{\circ} 52^{\prime} 29.0^{\prime \prime}$ & $82^{\circ} 52^{\prime} 37.7^{\prime \prime}$ \\
\hline 20 & 300.8 & 14.7 & $29^{\circ} 52^{\prime} 28.9^{\prime \prime}$ & $82^{\circ} 52^{\prime} 37.2^{\prime \prime}$ \\
\hline 21 & 310.7 & 9.9 & $29^{\circ} 52^{\prime} 28.8^{\prime \prime}$ & $82^{\circ} 52^{\prime} 36.8^{\prime \prime}$ \\
\hline
\end{tabular}




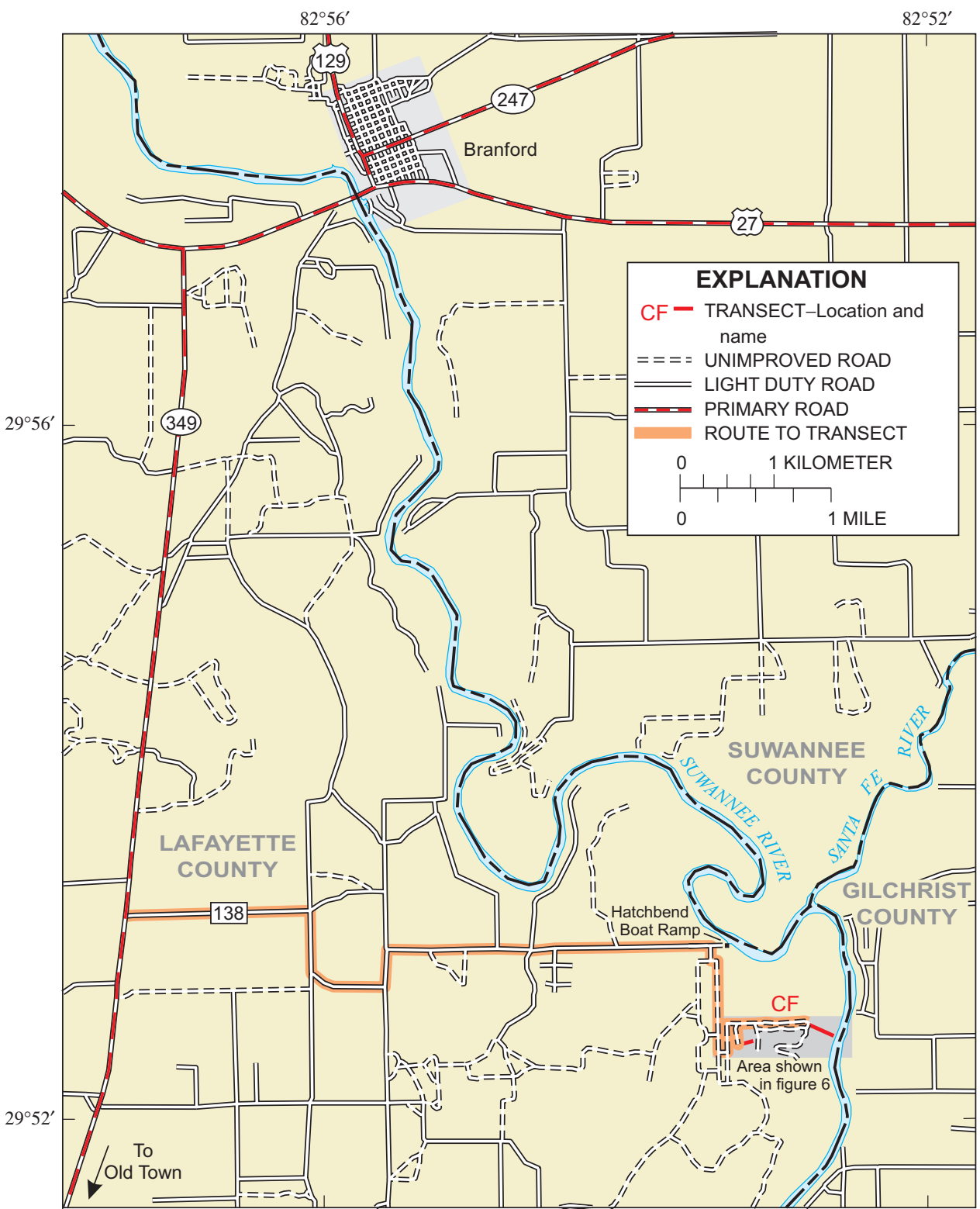

Figure 5. CF transect and surrounding area in the lower Suwannee River floodplain, Florida.

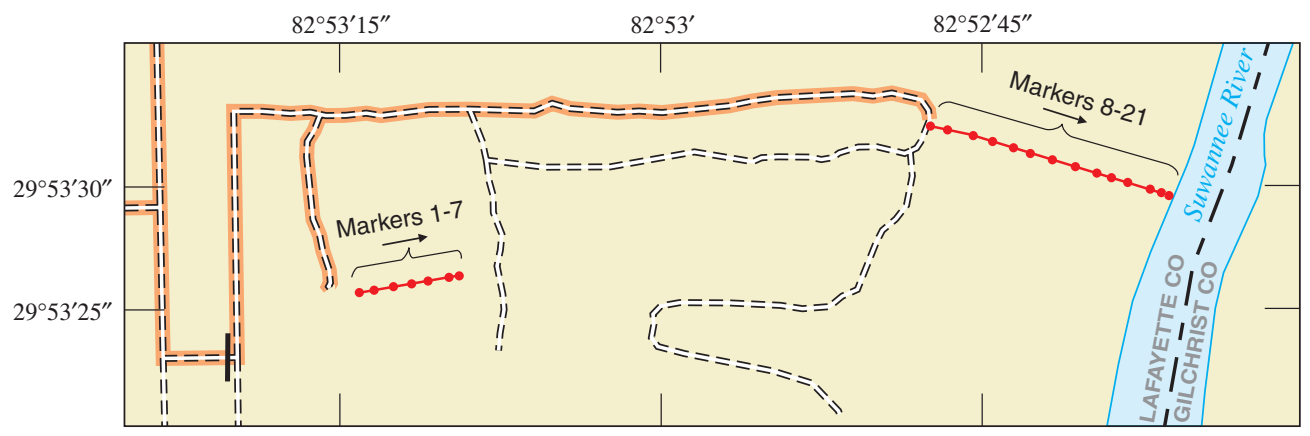

\begin{tabular}{|c|c|}
\hline & EXPLANATION \\
\hline$\rightarrow$ & $\begin{array}{l}\text { TRANSECT-Points indicate } \\
\text { marker locations }\end{array}$ \\
\hline & $\begin{array}{l}\text { LOCKED GATE-Maintained } \\
\text { by SRWMD }\end{array}$ \\
\hline$\longrightarrow$ & $\begin{array}{l}\text { INDICATES DIRECTION OF } \\
\text { INCREASING MARKER } \\
\text { NUMBERS }\end{array}$ \\
\hline$====$ & $\begin{array}{l}\text { UNIMPROVED ROADS } \\
\text { ROUTE TO TRANSECT }\end{array}$ \\
\hline 0 & $0.1 \quad 0.2$ KILOMETERS \\
\hline 0 & $0.1 \quad 0.2$ MILES \\
\hline
\end{tabular}

Figure 6. CF transect with marker locations and access roads in the lower Suwannee River floodplain, Florida. 
$\mathrm{CF}$ has the highest elevation and greatest topographic relief of all the transects. The elevations along the transect line range from 3.7 to $7.3 \mathrm{~m}$ above sea level (fig. 7). Natural riverbank levees (as seen at marker 20 on this transect) are prominent fea- tures on transects in the lower Suwannee River study area from rkm 104 to approximately rkm 43.

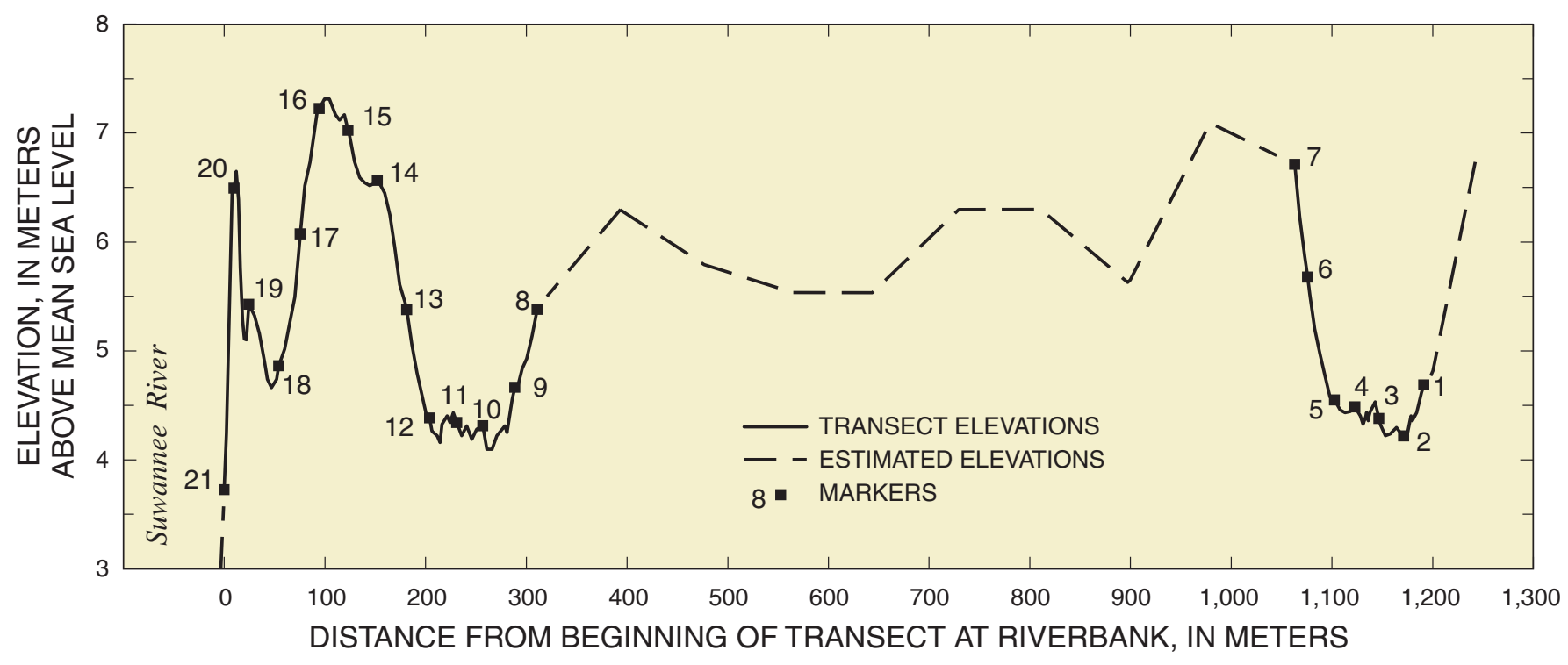

Figure 7. Land-surface elevations and marker locations at CF transect in the lower Suwannee River floodplain, Florida.

\section{Log Landing (LL)}

\section{LL transect is located on} SRWMD lands. Data collection efforts at this site should be coordinated with the SRWMD land manager in Live Oak, Florida at (800) 226-1066.

LL transect is located in eastern Dixie County, across the river from the town of Wannee, Florida, in Gilchrist County. The only access to LL transect is by river. The two closest public boat ramps to this site are Eula Landing boat ramp and a ramp in Wannee, Florida. The Wannee ramp was not used during the study period because it was in poor condition at the time. To reach Eula Landing from Fanning Springs, Florida, travel north on State Highway 26 to County Road 232 (fig. 8). Go north on County Road 232. When this paved road makes a $90^{\circ}$ turn to the right after about 5.8 miles, continue straight on the dirt road to SW $25^{\text {th }}$ Street. Go west to the Eula Landing boat ramp.

LL transect is $921.2 \mathrm{~m}$ in length and has a compass bearing of approximately $0^{\circ}$ from marker 22 (fig. 9). The latitude and longitude of each marker on the transect line are provided in table 3.

Land-surface elevations along the transect ranged from 2.4 to $5.3 \mathrm{~m}$ above sea level (fig. 10). A small open-water pond is visible to the west of this transect near marker 30 and a small flowing stream is located on the transect near marker 36. Both of these water features may be dry during a drought. Elevations between the edge of the river and the beginning of the transect (marker 22) and from the end of the transect (marker 51) to the area surrounding a verification plot were estimated in the field. The verification plot location and elevations are included in this report because data from this plot was used in conjunction with the LL transect data to provide information on a forest type not found on the transect line. 


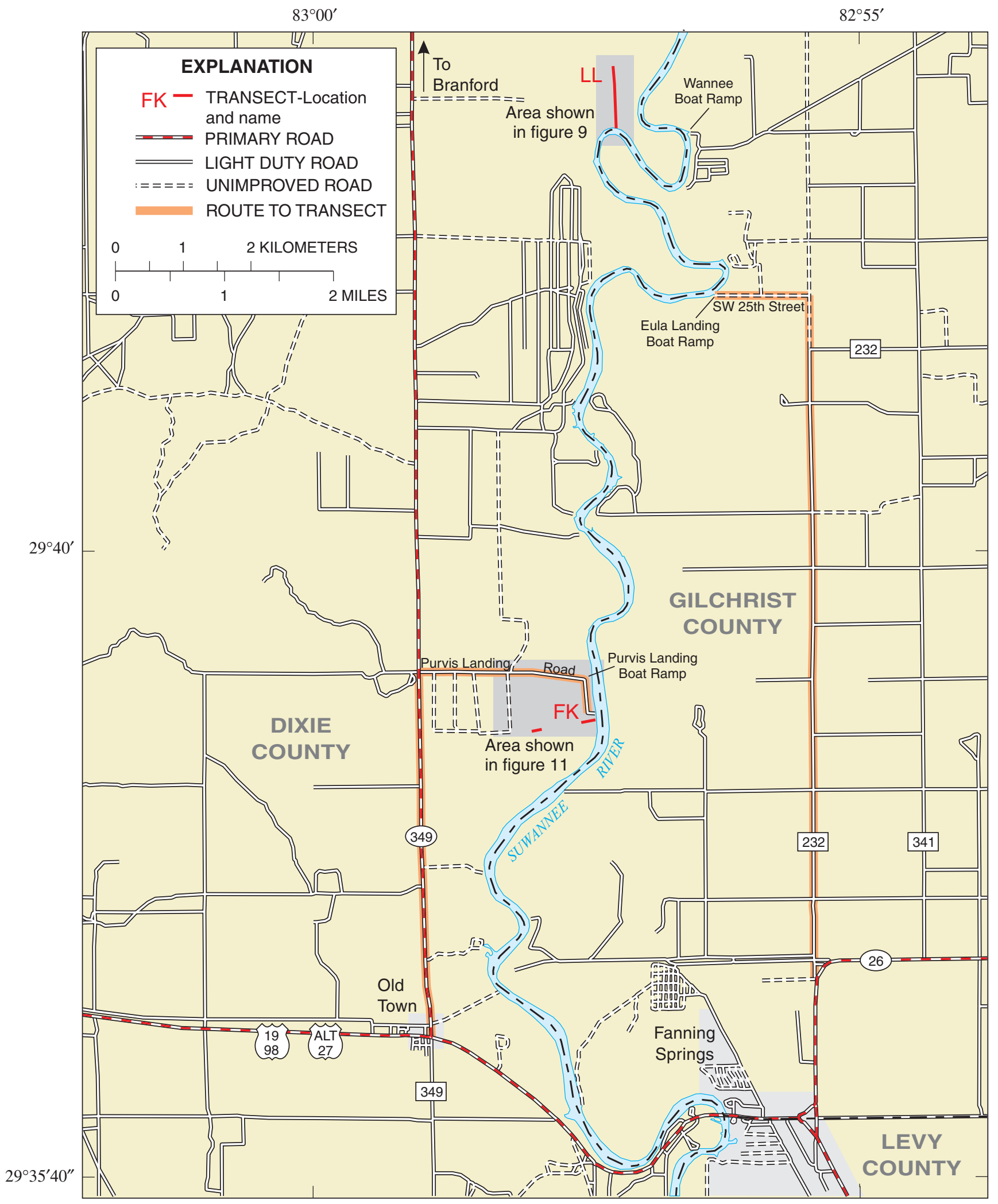

Figure 8. LL and FK transect locations with surrounding area in the lower Suwannee River floodplain, Florida. 


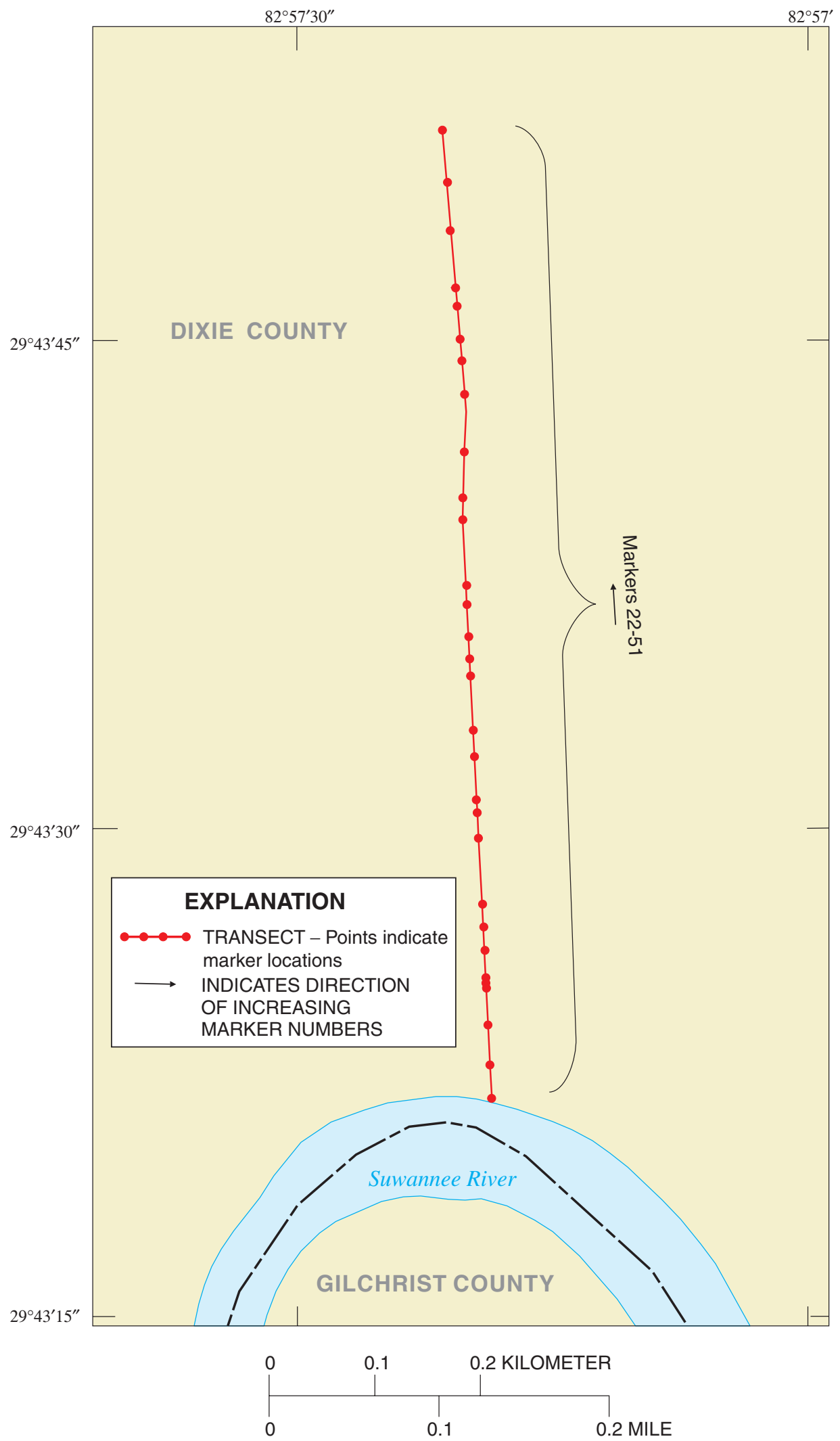

Figure 9. LL transect with marker locations and immediate vicinity in the lower Suwannee River floodplain, Florida. 
Table 3. Marker locations on LL transect in the lower Suwannee River floodplain, Florida

\begin{tabular}{|c|c|c|c|c|}
\hline $\begin{array}{l}\text { Marker } \\
\text { number }\end{array}$ & $\begin{array}{c}\text { Distance from } \\
\text { beginning of } \\
\text { transect }\end{array}$ & $\begin{array}{l}\text { Distance from } \\
\text { previous } \\
\text { marker }\end{array}$ & Latitude & Longitude \\
\hline 22 & 0.0 & 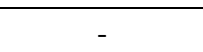 & $29^{\circ} 43^{\prime} 20.9^{\prime \prime}$ & $82^{\circ} 57^{\prime} 13.7^{\prime \prime}$ \\
\hline 23 & 31.8 & 31.8 & $29^{\circ} 43^{\prime} 21.9^{\prime \prime}$ & $82^{\circ} 57^{\prime} 13.8^{\prime \prime}$ \\
\hline 24 & 69.2 & 37.4 & $29^{\circ} 43^{\prime} 23.1^{\prime \prime}$ & $82^{\circ} 57^{\prime} 13.8^{\prime \prime}$ \\
\hline 25 & 104.5 & 35.3 & $29^{\circ} 43^{\prime} 24.3^{\prime \prime}$ & $82^{\circ} 57^{\prime} 13.9^{\prime \prime}$ \\
\hline 26 & 110.6 & 6.1 & $29^{\circ} 43^{\prime} 24.5^{\prime \prime}$ & $82^{\circ} 57^{\prime} 13.9^{\prime \prime}$ \\
\hline 27 & 113.6 & 3.0 & $29^{\circ} 43^{\prime} 24.6^{\prime \prime}$ & $82^{\circ} 57^{\prime} 13.9^{\prime \prime}$ \\
\hline 28 & 140.3 & 26.7 & $29^{\circ} 43^{\prime} 25.4^{\prime \prime}$ & $82^{\circ} 57^{\prime} 14.0^{\prime \prime}$ \\
\hline 29 & 162.9 & 22.6 & $29^{\circ} 43^{\prime} 26.1^{\prime \prime}$ & $82^{\circ} 57^{\prime} 14.1^{\prime \prime}$ \\
\hline 30 & 184.2 & 21.3 & $29^{\circ} 43^{\prime} 26.8^{\prime \prime}$ & $82^{\circ} 57^{\prime} 14.0^{\prime \prime}$ \\
\hline 31 & 246.2 & 62.0 & $29^{\circ} 43^{\prime} 28.9^{\prime \prime}$ & $82^{\circ} 57^{\prime} 14.1^{\prime \prime}$ \\
\hline 32 & 270.7 & 24.5 & $29^{\circ} 43^{\prime} 29.6^{\prime \prime}$ & $82^{\circ} 57^{\prime} 14.2^{\prime \prime}$ \\
\hline 33 & 282.7 & 12.0 & $29^{\circ} 43^{\prime} 30.0^{\prime \prime}$ & $82^{\circ} 57^{\prime} 14.2^{\prime \prime}$ \\
\hline 34 & 324.2 & 41.5 & $29^{\circ} 43^{\prime} 31.4^{\prime \prime}$ & $82^{\circ} 57^{\prime} 14.3^{\prime \prime}$ \\
\hline 35 & 349.2 & 25.0 & $29^{\circ} 43^{\prime} 32.2^{\prime \prime}$ & $82^{\circ} 57^{\prime} 14.3^{\prime \prime}$ \\
\hline 36 & 400.3 & 51.1 & $29^{\circ} 43^{\prime} 33.9^{\prime \prime}$ & $82^{\circ} 57^{\prime} 14.4^{\prime \prime}$ \\
\hline 37 & 417.0 & 16.7 & $29^{\circ} 43^{\prime} 34.4^{\prime \prime}$ & $82^{\circ} 57^{\prime} 14.5^{\prime \prime}$ \\
\hline 38 & 437.7 & 20.7 & $29^{\circ} 43^{\prime} 35.1^{\prime \prime}$ & $82^{\circ} 57^{\prime} 14.5^{\prime \prime}$ \\
\hline 39 & 467.7 & 30.0 & $29^{\circ} 43^{\prime} 36.0^{\prime \prime}$ & $82^{\circ} 57^{\prime} 14.6^{\prime \prime}$ \\
\hline 40 & 486.3 & 18.6 & $29^{\circ} 43^{\prime} 36.6^{\prime \prime}$ & $82^{\circ} 57^{\prime} 14.6^{\prime \prime}$ \\
\hline 41 & 548.2 & 61.9 & $29^{\circ} 43^{\prime} 38.6^{\prime \prime}$ & $82^{\circ} 57^{\prime} 14.7^{\prime \prime}$ \\
\hline 42 & 569.0 & 20.8 & $29^{\circ} 43^{\prime} 39.3^{\prime \prime}$ & $82^{\circ} 57^{\prime} 14.7^{\prime \prime}$ \\
\hline 43 & 612.6 & 43.6 & $29^{\circ} 43^{\prime} 40.7^{\prime \prime}$ & $82^{\circ} 57^{\prime} 14.7^{\prime \prime}$ \\
\hline 44 & 667.1 & 54.5 & $29^{\circ} 43^{\prime} 42.5^{\prime \prime}$ & $82^{\circ} 57^{\prime} 14.6^{\prime \prime}$ \\
\hline 45 & 699.0 & 31.9 & $29^{\circ} 43^{\prime} 43.5^{\prime \prime}$ & $82^{\circ} 57^{\prime} 14.7^{\prime \prime}$ \\
\hline 46 & 719.3 & 20.3 & $29^{\circ} 43^{\prime} 44.2^{\prime \prime}$ & $82^{\circ} 57^{\prime} 14.8^{\prime \prime}$ \\
\hline 47 & 750.6 & 31.3 & $29^{\circ} 43^{\prime} 45.2^{\prime \prime}$ & $82^{\circ} 57^{\prime} 14.9^{\prime \prime}$ \\
\hline 48 & 768.0 & 17.4 & $29^{\circ} 43^{\prime} 45.9^{\prime \prime}$ & $82^{\circ} 57^{\prime} 14.9^{\prime \prime}$ \\
\hline 49 & 822.4 & 54.4 & $29^{\circ} 43^{\prime} 47.5^{\prime \prime}$ & $82^{\circ} 57^{\prime} 15.1^{\prime \prime}$ \\
\hline 50 & 868.2 & 45.8 & $29^{\circ} 43^{\prime} 49.0^{\prime \prime}$ & $82^{\circ} 57^{\prime} 15.3^{\prime \prime}$ \\
\hline 51 & 916.6 & 48.4 & $29^{\circ} 43^{\prime} 50.6^{\prime \prime}$ & $82^{\circ} 57^{\prime} 15.4^{\prime \prime}$ \\
\hline
\end{tabular}

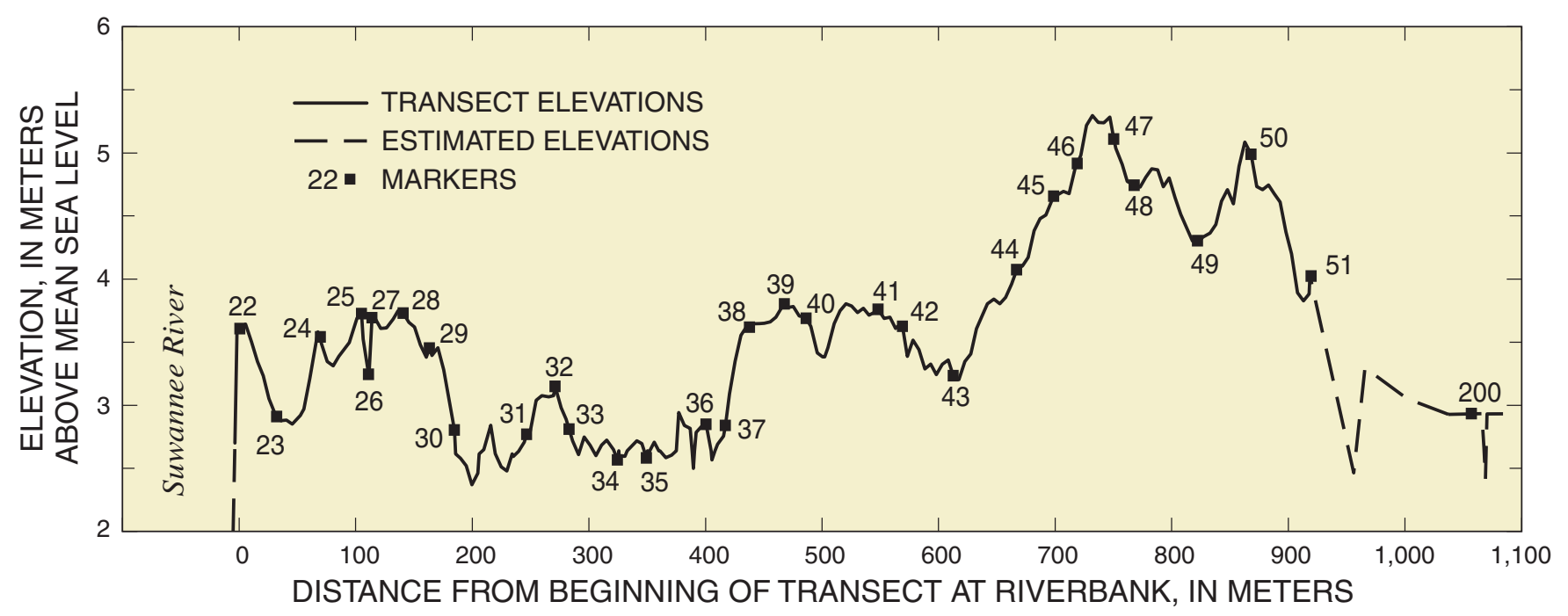

Figure 10. Land-surface elevations and marker locations at LL transect in the lower Suwannee River floodplain, Florida. 
FK transect is comprised of two segments, the east segment (markers 52-58) and the west segment (markers 59-66) (fig. 11). The transect is located on SRWMD lands except for three markers (6466) on the west segment of the transect that are located on private property. The easiest land access to all markers on the west segment is also across private property. Landowner permission for permanent marker installation and access was granted at the time the markers were installed, but any further use of markers 64-66 or access across private property to the west segment will require permission from the current landowner. The SRWMD land manager in Live Oak, Florida should also be contacted at (800) 226-1066 prior to visiting this transect.
FK transect is located in eastern Dixie County, north-northeast of Old Town, Florida and west of the Suwannee River. To reach FK transect from Old Town, travel north on State Highway 349 approximately 3.4 miles and turn right onto Purvis Landing Road (fig. 8). This road ends at the boat ramp. Turn right on the last road before the boat ramp.
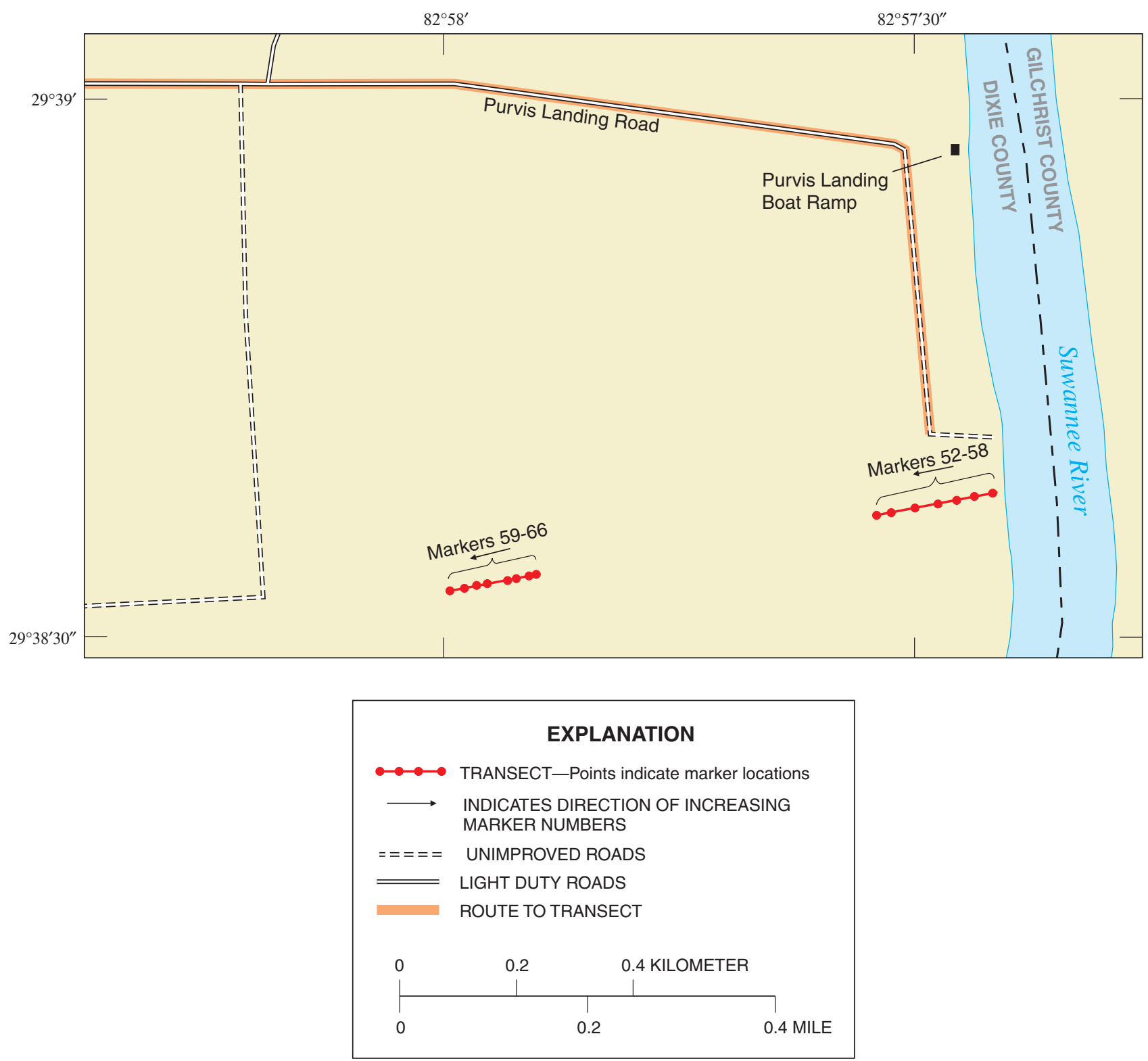

Figure 11. FK transect with marker locations and access roads in the lower Suwannee River floodplain, Florida. 
The east segment of the FK transect is 210.3 meters in length and has a compass bearing of $264^{\circ}$ from marker 52 at the river (fig.11). The west segment is 151.3 meters long and has a bearing of $79^{\circ}$ from marker 66. Latitude and longitude of each marker on the transect are provided in table 4 .

The highest elevation on this transect is on the riverbank levee on the east segment (fig.12). Elevations of the land connecting the east and west segments were estimated in the field and include a large slough near marker 59. This slough contains soft bottom sediments that make it difficult to walk across (except during prolonged drought).

Table 4. Marker locations on FK transect in the lower Suwannee River floodplain, Florida

\begin{tabular}{ccccc}
\hline $\begin{array}{c}\text { Marker } \\
\text { number }\end{array}$ & $\begin{array}{c}\text { Distance from } \\
\text { beginning of } \\
\text { segment }\end{array}$ & $\begin{array}{c}\text { Distance from } \\
\text { previous } \\
\text { marker }\end{array}$ & Latitude & Longitude \\
\hline \multicolumn{5}{c}{ East Segment } \\
\hline 52 & 0.0 & - & $29^{\circ} 38^{\prime} 37.2^{\prime \prime}$ & $82^{\circ} 57^{\prime} 25.6^{\prime \prime}$ \\
53 & 40.0 & 40.0 & $29^{\circ} 38^{\prime} 37.0^{\prime \prime}$ & $82^{\circ} 57^{\prime} 26.7^{\prime \prime}$ \\
54 & 72.0 & 32.0 & $29^{\circ} 38^{\prime} 36.8^{\prime \prime}$ & $82^{\circ} 57^{\prime}, 27.9^{\prime \prime}$ \\
55 & 103.6 & 31.6 & $29^{\circ} 38^{\prime}, 36.6^{\prime \prime}$ & $82^{\circ} 57^{\prime} 29.0^{\prime \prime}$ \\
56 & 144.1 & 40.5 & $29^{\circ} 38^{\prime} 36.4^{\prime \prime}$ & $82^{\circ} 57^{\prime}, 30.5^{\prime \prime}$ \\
57 & 185.3 & 41.2 & $29^{\circ} 38^{\prime} 36.1^{\prime \prime}$ & $82^{\circ} 57^{\prime} 32.0^{\prime \prime}$ \\
58 & 210.3 & 25.0 & $29^{\circ} 38^{\prime} 36.0^{\prime \prime}$ & $82^{\circ} 57^{\prime} 33.0^{\prime \prime}$ \\
\hline 66 & 0.0 & - & $29^{\circ} 38^{\prime} 31.8^{\prime \prime}$ & $82^{\circ} 58^{\prime}, 00.2^{\prime \prime}$ \\
65 & 26.1 & 26.1 & $29^{\circ} 38^{\prime} 31.9^{\prime \prime}$ & $82^{\circ} 57^{\prime} 59.3^{\prime \prime}$ \\
64 & 47.6 & 21.5 & $29^{\circ} 38^{\prime}, 32.0^{\prime \prime}$ & $82^{\circ} 57^{\prime} 58.5^{\prime \prime}$ \\
63 & 68.1 & 20.5 & $29^{\circ} 38^{\prime} 32.2^{\prime \prime}$ & $82^{\circ} 57^{\prime} 57.7^{\prime \prime}$ \\
62 & 101.2 & 33.1 & $29^{\circ} 38^{\prime} 32.4^{\prime \prime}$ & $82^{\circ} 57^{\prime} 56.5^{\prime \prime}$ \\
61 & 117.6 & 16.4 & $29^{\circ} 38^{\prime} 32.5^{\prime \prime}$ & $82^{\circ} 57^{\prime} 55.9^{\prime \prime}$ \\
60 & 138.8 & 21.2 & $29^{\circ} 38^{\prime}, 32.6^{\prime \prime}$ & $82^{\circ} 57^{\prime} 55.1^{\prime \prime}$ \\
59 & 151.3 & 12.5 & $29^{\circ} 38^{\prime} 32.7^{\prime \prime}$ & $82^{\circ} 57^{\prime} 54.7^{\prime \prime}$ \\
\hline
\end{tabular}

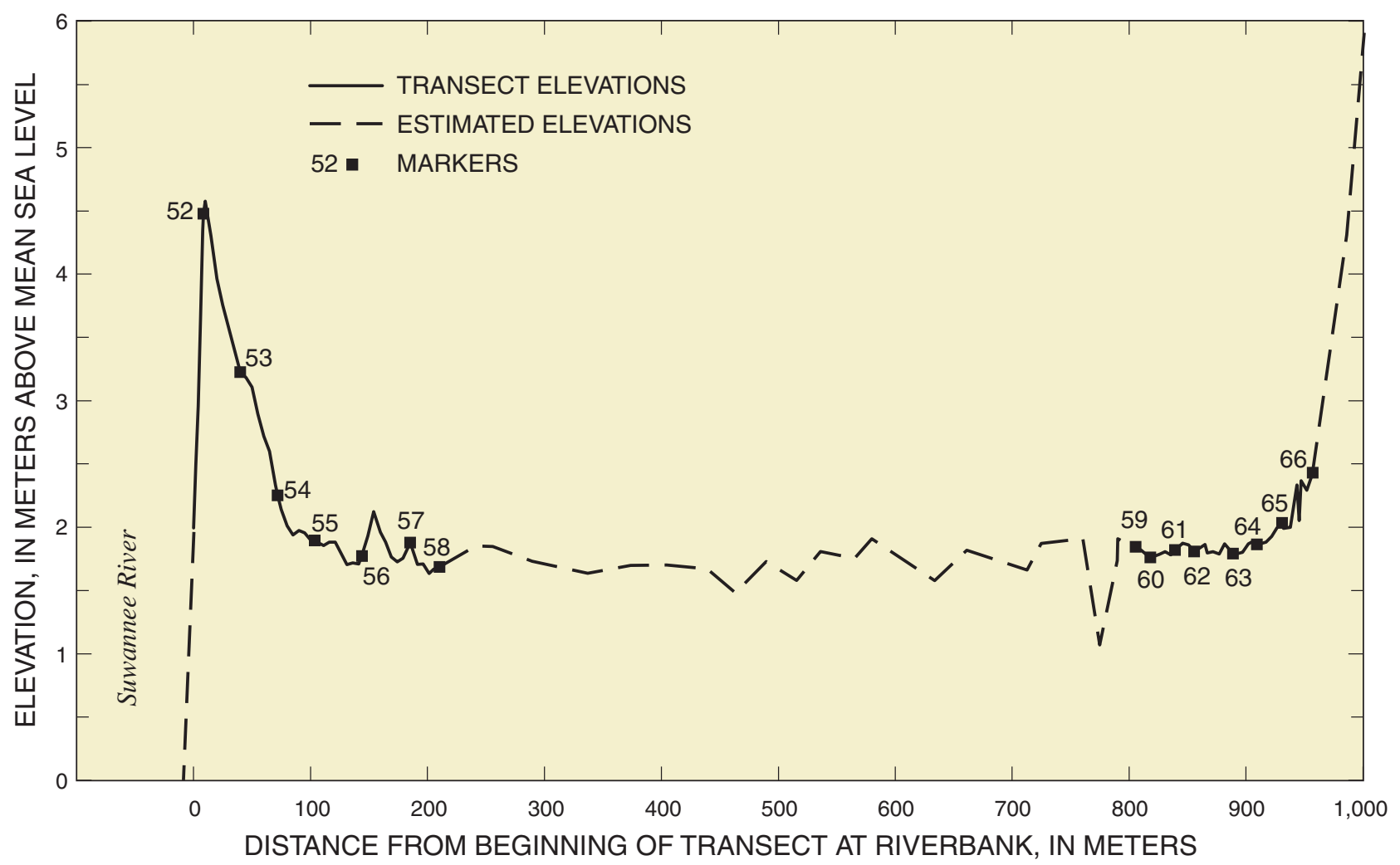

Figure 12. Land-surface elevations and marker locations at FK transect in the lower Suwannee River floodplain, Florida. 
MS transect is located in Manatee Springs State Park. Contact the Park Manager at (352) 4936738 in advance of field visits for coordination and arrangements. A research/collecting permit is needed to conduct a study in a Florida state park or preserve. Permit applications are available from the Florida Department of Environmental Protection, Division of Recreation and Parks in Gainesville, Florida at (352) 955-2135.

Manatee Springs State Park is located in northwestern Levy County, Florida. To reach the park from Chiefland, Florida, go west on State Highway 320 directly to the park entrance (fig. 13). The

transect is located in the north end trail system; the trail entrance is the second road on the right after the park entrance (fig. 14). Although the trail system is open to foot and bicycle traffic during park hours, a locked gate restricts motor vehicle access. From the trail entrance, follow Scenic trail approximately 0.9 miles to a T-intersection then turn right on Clay trail. After a very short distance, turn left onto Hardwood trail and follow this trail approximately 0.4 miles.

MS transect measures 1,009 meters in length and is the longest of the 12 transects. The compass bearing of the transect line is $316.5^{\circ}$ from marker 67 to marker $82,338.5^{\circ}$ from marker 82 to 83 , and $353^{\circ}$ from marker 83 to the transect endpoint at marker 94 . The latitude and longitude of each marker are listed in table 5 .

Land-surface elevations along the transect line are illustrated in figure 15. Similar to $\mathrm{CF}$ transect, there is a second higher ridge on this transect immediately behind the riverbank levee. The swamps between markers 72-81 and 84-88 are continuously saturated and the ground is often "soupy".

Table 5. Marker locations on MS transect in the lower Suwannee River floodplain, Florida

\begin{tabular}{|c|c|c|c|c|}
\hline $\begin{array}{c}\text { Marker } \\
\text { number }\end{array}$ & $\begin{array}{l}\text { Distance from } \\
\text { beginning of } \\
\text { transect }\end{array}$ & $\begin{array}{l}\text { Distance from } \\
\text { previous } \\
\text { marker }\end{array}$ & Latitude & Longitude \\
\hline 67 & 0.0 & - & $29^{\circ} 30^{\prime} 37.1^{\prime \prime}$ & $82^{\circ} 58^{\prime} 27.8^{\prime \prime}$ \\
\hline 68 & 48.2 & 48.2 & $29^{\circ} 30^{\prime} 38.2^{\prime \prime}$ & $82^{\circ} 58^{\prime} 29.2^{\prime \prime}$ \\
\hline 69 & 102.6 & 54.4 & $29^{\circ} 30^{\prime} 39.4^{\prime \prime}$ & $82^{\circ} 58^{\prime} 30.7^{\prime \prime}$ \\
\hline 70 & 150.5 & 47.9 & $29^{\circ} 30^{\prime} 40.4^{\prime \prime}$ & $82^{\circ} 58^{\prime} 32.0^{\prime \prime}$ \\
\hline 71 & 200.0 & 49.5 & $29^{\circ} 30^{\prime} 41.5^{\prime \prime}$ & $82^{\circ} 58^{\prime} 33.3^{\prime \prime}$ \\
\hline 72 & 238.1 & 38.1 & $29^{\circ} 30^{\prime} 42.3^{\prime \prime}$ & $82^{\circ} 58^{\prime} 34.4^{\prime \prime}$ \\
\hline 73 & 261.0 & 22.9 & $29^{\circ} 30^{\prime} 42.8^{\prime \prime}$ & $82^{\circ} 58^{\prime} 35.0^{\prime \prime}$ \\
\hline 74 & 291.5 & 30.5 & $29^{\circ} 30^{\prime} 43.5^{\prime \prime}$ & $82^{\circ} 58^{\prime} 35.9^{\prime \prime}$ \\
\hline 75 & 325.4 & 33.9 & $29^{\circ} 30^{\prime} 44.2^{\prime \prime}$ & $82^{\circ} 58^{\prime} 36.8^{\prime \prime}$ \\
\hline 76 & 358.7 & 33.3 & $29^{\circ} 30^{\prime} 44.9^{\prime \prime}$ & $82^{\circ} 58^{\prime} 37.7^{\prime \prime}$ \\
\hline 77 & 391.7 & 33.0 & $29^{\circ} 30^{\prime} 45.6^{\prime \prime}$ & $82^{\circ} 58^{\prime} 38.6^{\prime \prime}$ \\
\hline 78 & 420.7 & 29.0 & $29^{\circ} 30^{\prime} 46.3^{\prime \prime}$ & $82^{\circ} 58^{\prime} 39.4^{\prime \prime}$ \\
\hline 79 & 452.7 & 32.0 & $29^{\circ} 30^{\prime} 47.0^{\prime \prime}$ & $82^{\circ} 58^{\prime} 40.3^{\prime \prime}$ \\
\hline 80 & 475.4 & 22.7 & $29^{\circ} 30^{\prime} 47.5^{\prime \prime}$ & $82^{\circ} 58^{\prime} 40.9^{\prime \prime}$ \\
\hline 81 & 515.4 & 40.0 & $29^{\circ} 30^{\prime} 48.3^{\prime \prime}$ & $82^{\circ} 58^{\prime} 42.0^{\prime \prime}$ \\
\hline 82 & 553.1 & 37.7 & $29^{\circ} 30^{\prime} 49.2^{\prime \prime}$ & $82^{\circ} 58^{\prime} 43.1^{\prime \prime}$ \\
\hline 83 & 587.7 & 34.6 & $29^{\circ} 30^{\prime} 50.2^{\prime \prime}$ & $82^{\circ} 58^{\prime} 43.7^{\prime \prime}$ \\
\hline 84 & 639.2 & 51.5 & $29^{\circ} 30^{\prime} 51.8^{\prime \prime}$ & $82^{\circ} 58^{\prime} 44.0^{\prime \prime}$ \\
\hline 85 & 676.2 & 37.0 & $29^{\circ} 30^{\prime} 53.0^{\prime \prime}$ & $82^{\circ} 58^{\prime} 44.2^{\prime \prime}$ \\
\hline 86 & 704.0 & 27.8 & $29^{\circ} 30^{\prime} 53.9^{\prime \prime}$ & $82^{\circ} 58^{\prime} 44.3^{\prime \prime}$ \\
\hline 87 & 737.8 & 33.8 & $29^{\circ} 30^{\prime} 55.0^{\prime \prime}$ & $82^{\circ} 58^{\prime} 44.5^{\prime \prime}$ \\
\hline 88 & 774.0 & 36.2 & $29^{\circ} 30^{\prime} 56.1^{\prime \prime}$ & $82^{\circ} 58^{\prime} 44.7^{\prime \prime}$ \\
\hline 89 & 804.3 & 30.3 & $29^{\circ} 30^{\prime} 57.1^{\prime \prime}$ & $82^{\circ} 58^{\prime} 44.8^{\prime \prime}$ \\
\hline 90 & 845.0 & 40.7 & $29^{\circ} 30^{\prime} 58.4^{\prime \prime}$ & $82^{\circ} 58^{\prime} 45.1^{\prime \prime}$ \\
\hline 91 & 889.5 & 44.5 & $29^{\circ} 30^{\prime} 59.9^{\prime \prime}$ & $82^{\circ} 58^{\prime} 45.3^{\prime \prime}$ \\
\hline 92 & 917.4 & 27.9 & $29^{\circ} 31^{\prime} 00.8^{\prime \prime}$ & $82^{\circ} 58^{\prime} 45.4^{\prime \prime}$ \\
\hline 93 & 970.1 & 52.7 & $29^{\circ} 31^{\prime} 02.5^{\prime \prime}$ & $82^{\circ} 58^{\prime} 45.7^{\prime \prime}$ \\
\hline 94 & 1003.7 & 33.6 & $29^{\circ} 31^{\prime} 03.5^{\prime \prime}$ & $82^{\circ} 58^{\prime} 45.9^{\prime \prime}$ \\
\hline
\end{tabular}


$83^{\circ} 00^{\prime}$

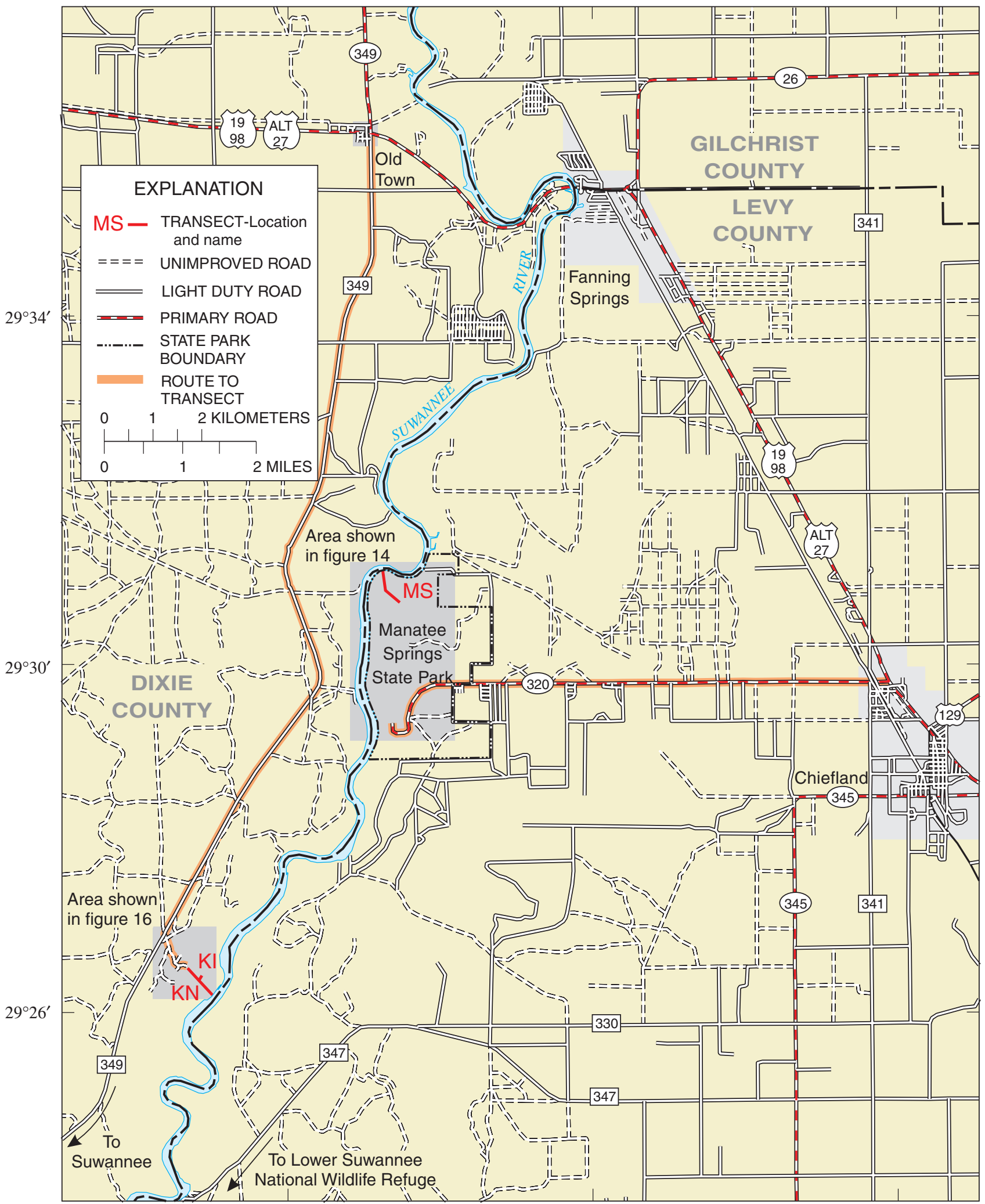

Figure 13. $\mathrm{MS}, \mathrm{KN}$ and $\mathrm{KI}$ transect locations and surrounding area in the lower Suwannee River floodplain, Florida. 


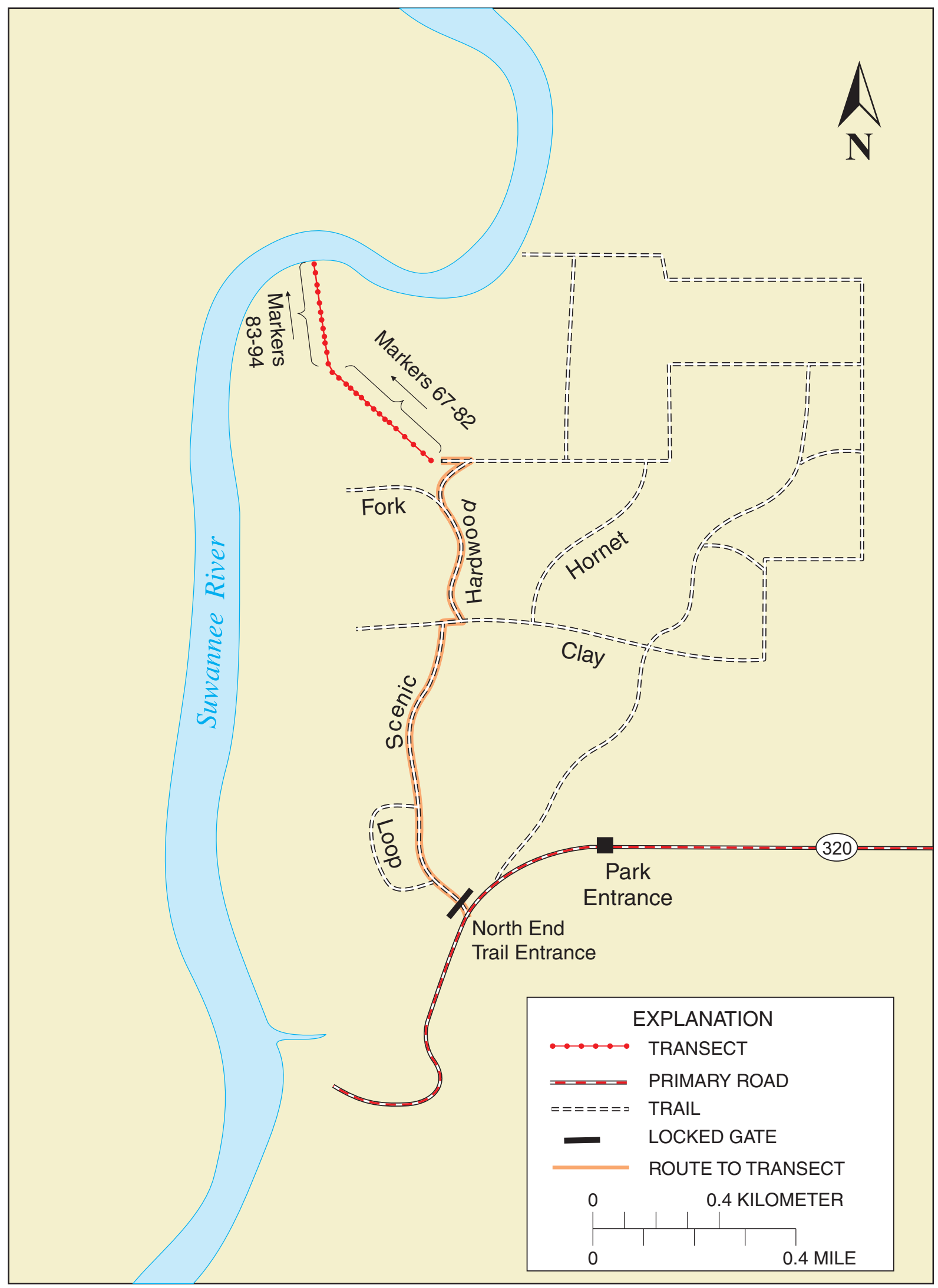

Figure 14. MS transect with marker locations and access roads in the lower Suwannee River floodplain, Florida. 


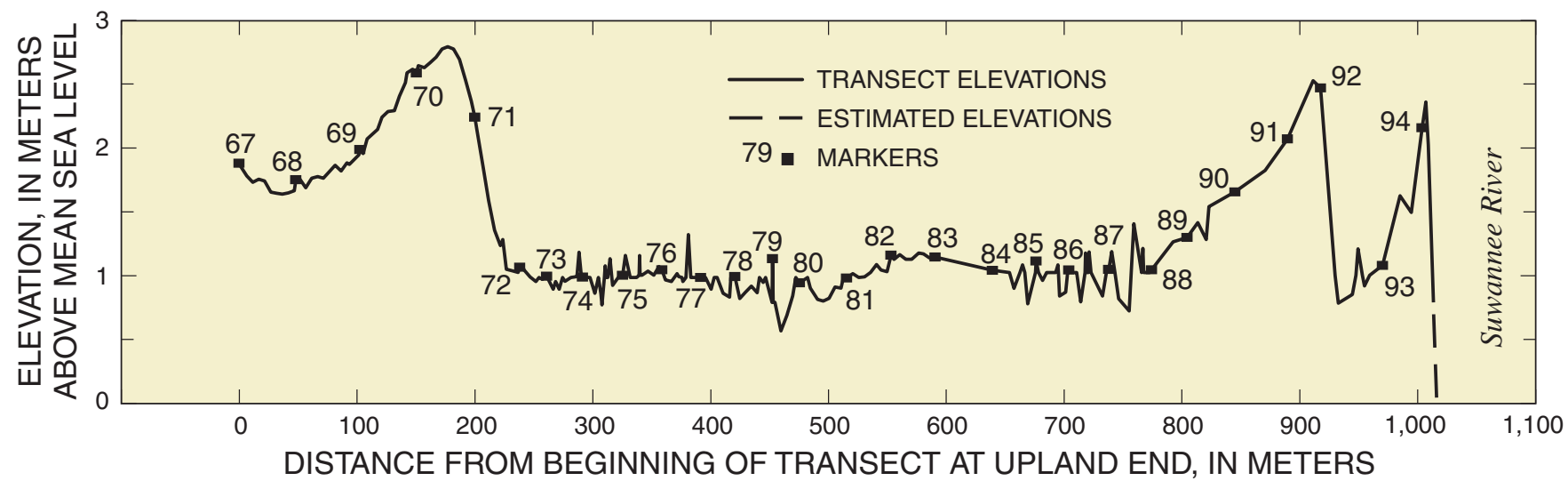

Figure 15. Land-surface elevations and marker locations at MS transect in the lower Suwannee River floodplain, Florida.

\section{Keen and Keen Island (KN and KI)}

$\mathrm{KN}$ and $\mathrm{KI}$ transects are located in the Lower Suwannee National Wildlife Refuge (LSNWR). Road access to this transect is restricted by a locked gate, therefore arrangements must be made with the Refuge Manager at (352) 493-0238 prior to field visits. The LSNWR Headquarters are located approximately 2 miles south of Fowlers Bluff, Florida on Highway 347.

$\mathrm{KN}$ and $\mathrm{KI}$ transects are located in southeastern Dixie County, west of the Suwannee River. To reach these transects from Old Town, Florida, go south on
County Road 349 (fig. 13). Travel 11.5 miles and turn left on the dirt road with a yellow gate at the entrance. Follow this road (fig. 16) until it forks; continue straight ahead (left fork) to a locked gate, proceed to the split in the road, take the right fork, then travel a short distance and take the left fork. Follow this road to the dead end.

$\mathrm{KN}$ transect is $734.1 \mathrm{~m}$ long and has a compass bearing of $140^{\circ}$ from marker 95 . The latitude and longitude of each marker are listed in table $6 . \mathrm{KI}$ is a $100 \mathrm{~m}$ transect that lies nearly perpendicular to $\mathrm{KN}$. The beginning of KI is located between KN markers 106 and 107 and this transect has a compass bearing of $30^{\circ}$ from marker 122 . The latitude and longitude of each marker on this transect are listed in table 7.

Land-surface elevations of the transects are presented in figures 17 and 18. The lowest elevations on $\mathrm{KN}$ transect occur in a marsh that lies between markers 120 and 121, and in a tidal creek near marker 114. Soils on most of the $\mathrm{KN}$ transect are continuously saturated mucks; however the soils on KI are usually dry and sandy. 


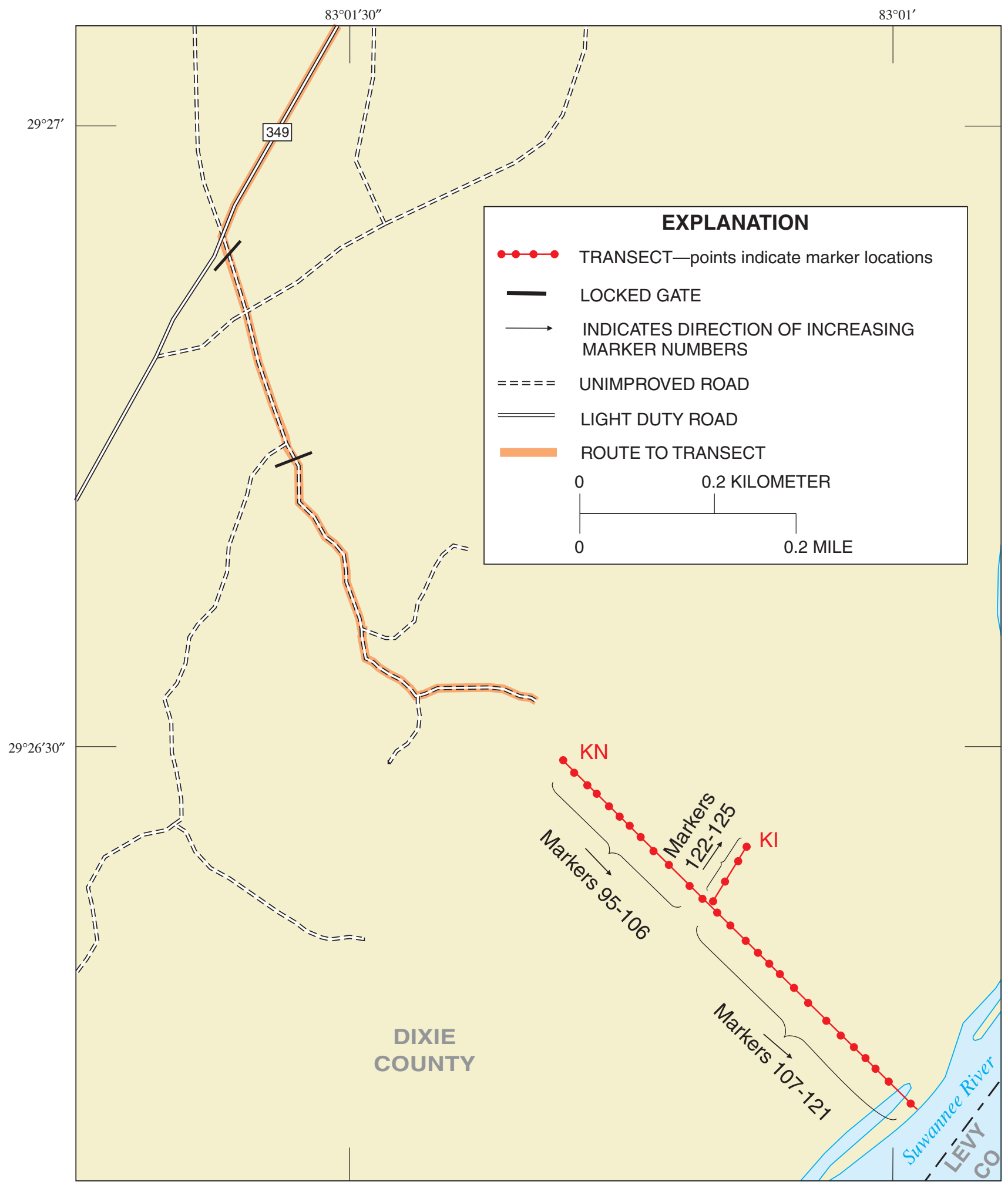

Figure 16. $\mathrm{KN}$ and $\mathrm{KI}$ transects with marker locations and access roads in the lower Suwannee River floodplain, Florida. 


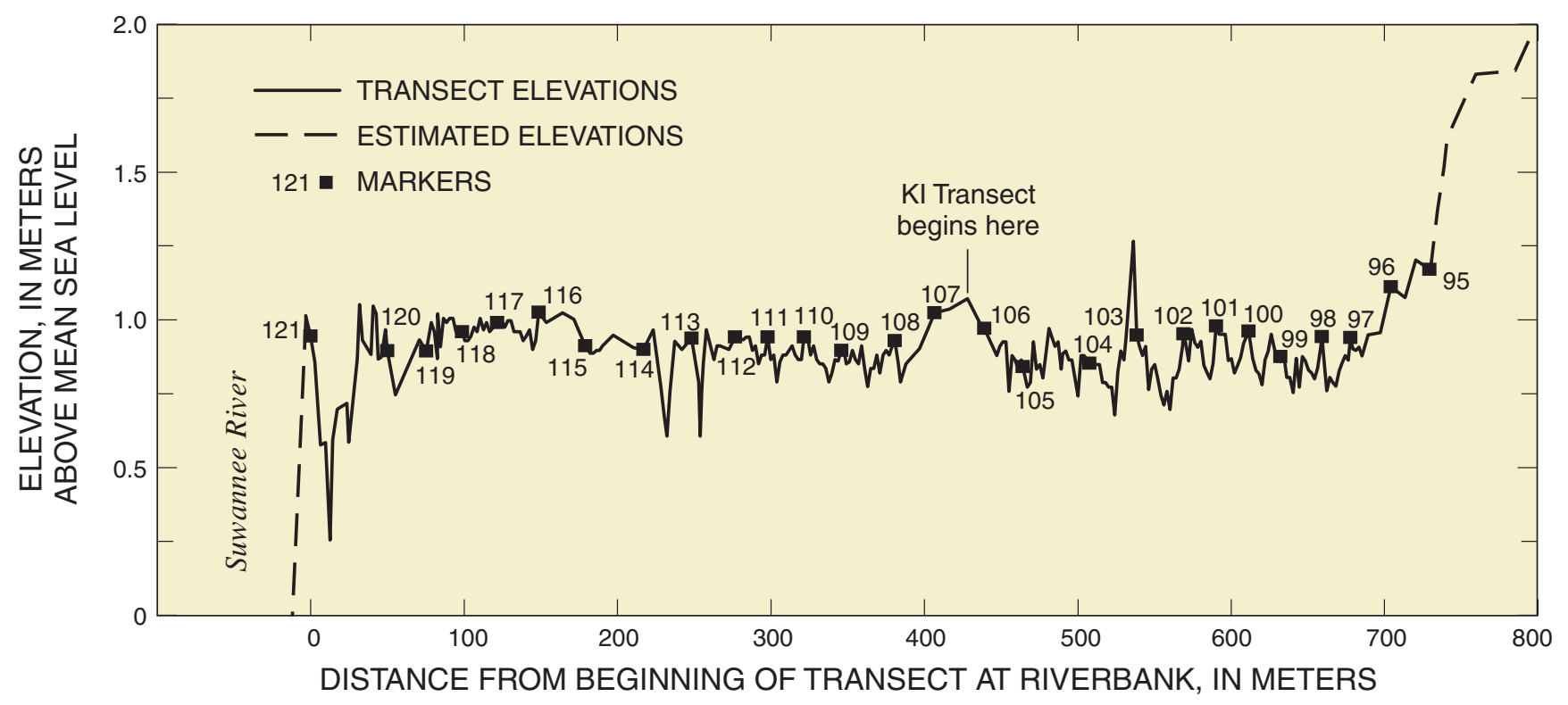

Figure 17. Land-surface elevations and marker locations at KN transect in the lower Suwannee River Floodplain, Florida.

Table 6. Marker locations on $\mathrm{KN}$ transect in the lower Suwannee River floodplain, Florida

\begin{tabular}{|c|c|c|c|c|}
\hline $\begin{array}{l}\text { Marker } \\
\text { number }\end{array}$ & $\begin{array}{l}\text { Distance } \\
\text { from } \\
\text { beginning } \\
\text { of transect }\end{array}$ & $\begin{array}{c}\text { Distance } \\
\text { from } \\
\text { previous } \\
\text { marker }\end{array}$ & Latitude & Longitude \\
\hline 95 & 0.0 & - & $29^{\circ} 26^{\prime} 28.5^{\prime \prime}$ & $83^{\circ} 01^{\prime} 18.8^{\prime \prime}$ \\
\hline 96 & 25.2 & 25.2 & $29^{\circ} 26^{\prime} 27.9^{\prime \prime}$ & $83^{\circ} 01^{\prime} 18.1^{\prime \prime}$ \\
\hline 97 & 51.6 & 26.4 & $29^{\circ} 26^{\prime} 27.3^{\prime \prime}$ & $83^{\circ} 01^{\prime} 17.4^{\prime \prime}$ \\
\hline 98 & 70.2 & 18.6 & $29^{\circ} 26^{\prime} 26.9^{\prime \prime}$ & $83^{\circ} 01^{\prime} 16.9^{\prime \prime}$ \\
\hline 99 & 97.2 & 27.0 & $29^{\circ} 26^{\prime} 26.3^{\prime \prime}$ & $83^{\circ} 01^{\prime} 16.2^{\prime \prime}$ \\
\hline 100 & 117.9 & 20.7 & $29^{\circ} 26^{\prime} 25.8^{\prime \prime}$ & $83^{\circ} 01^{\prime} 15.7^{\prime \prime}$ \\
\hline 101 & 139.1 & 21.2 & $29^{\circ} 26^{\prime} 25.3^{\prime \prime}$ & $83^{\circ} 01^{\prime} 15.1^{\prime \prime}$ \\
\hline 102 & 160.3 & 21.2 & $29^{\circ} 26^{\prime} 24.8^{\prime \prime}$ & $83^{\circ} 01^{\prime} 14.5^{\prime \prime}$ \\
\hline 103 & 190.8 & 30.5 & $29^{\circ} 26^{\prime} 24.1^{\prime \prime}$ & $83^{\circ} 01^{\prime} 13.7^{\prime \prime}$ \\
\hline 104 & 221.7 & 30.9 & $29^{\circ} 26^{\prime} 23.4^{\prime \prime}$ & $83^{\circ} 01^{\prime} 12.9^{\prime \prime}$ \\
\hline 105 & 265.2 & 43.5 & $29^{\circ} 26^{\prime} 22.4^{\prime \prime}$ & $83^{\circ} 01^{\prime} 11.8^{\prime \prime}$ \\
\hline 106 & 290.2 & 25.0 & $29^{\circ} 26^{\prime} 21.9^{\prime \prime}$ & $83^{\circ} 01^{\prime} 11.1^{\prime \prime}$ \\
\hline 107 & 322.9 & 32.7 & $29^{\circ} 26^{\prime} 21.1^{\prime \prime}$ & $83^{\circ} 01^{\prime} 10.3^{\prime \prime}$ \\
\hline 108 & 348.5 & 25.6 & $29^{\circ} 26^{\prime} 20.5^{\prime \prime}$ & $83^{\circ} 01^{\prime} 09.6^{\prime \prime}$ \\
\hline 109 & 383.3 & 34.8 & $29^{\circ} 26^{\prime} 19.7^{\prime \prime}$ & $83^{\circ} 01^{\prime} 08.6^{\prime \prime}$ \\
\hline 110 & 407.6 & 24.3 & $29^{\circ} 26^{\prime} 19.2^{\prime \prime}$ & $83^{\circ} 01^{\prime} 08.0^{\prime \prime}$ \\
\hline 111 & 431.3 & 23.7 & $29^{\circ} 26^{\prime} 18.7^{\prime \prime}$ & $83^{\circ} 01^{\prime} 07.4^{\prime \prime}$ \\
\hline 112 & 452.6 & 21.3 & $29^{\circ} 26^{\prime} 18.2^{\prime \prime}$ & $83^{\circ} 01^{\prime} 06.8^{\prime \prime}$ \\
\hline 113 & 481.0 & 28.4 & $29^{\circ} 26^{\prime} 17.5^{\prime \prime}$ & $83^{\circ} 01^{\prime} 06.1^{\prime \prime}$ \\
\hline 114 & 512.3 & 31.3 & $29^{\circ} 26^{\prime} 16.8^{\prime \prime}$ & $83^{\circ} 01^{\prime} 05.2^{\prime \prime}$ \\
\hline 115 & 550.3 & 38.0 & $29^{\circ} 26^{\prime} 15.9^{\prime \prime}$ & $83^{\circ} 01^{\prime} 04.2^{\prime \prime}$ \\
\hline 116 & 580.6 & 30.3 & $29^{\circ} 26^{\prime} 15.2^{\prime \prime}$ & $83^{\circ} 01^{\prime} 03.4^{\prime \prime}$ \\
\hline 117 & 607.5 & 26.9 & $29^{\circ} 26^{\prime} 14.6^{\prime \prime}$ & $83^{\circ} 01^{\prime} 02.7^{\prime \prime}$ \\
\hline 118 & 630.5 & 23.0 & $29^{\circ} 26^{\prime} 14.1^{\prime \prime}$ & $83^{\circ} 01^{\prime} 02.1^{\prime \prime}$ \\
\hline 119 & 653.8 & 23.3 & $29^{\circ} 26^{\prime} 13.6^{\prime \prime}$ & $83^{\circ} 01^{\prime} 01.5^{\prime \prime}$ \\
\hline 120 & 679.2 & 25.4 & $29^{\circ} 26^{\prime} 13.0^{\prime \prime}$ & $83^{\circ} 01^{\prime} 00.8^{\prime \prime}$ \\
\hline 121 & 729.3 & 50.1 & $29^{\circ} 26^{\prime} 11.9^{\prime \prime}$ & $83^{\circ} 01^{\prime} 59.5^{\prime \prime}$ \\
\hline
\end{tabular}

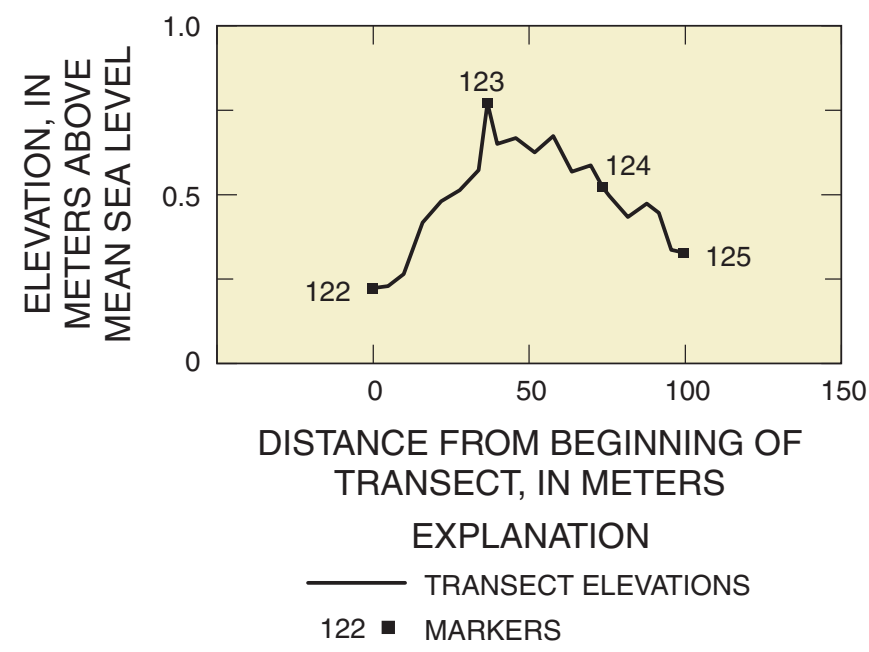

Figure 18. Land-surface elevations and marker locations at $\mathrm{KI}$ transect in the lower Suwannee River Floodplain, Florida.

Table 7. Marker locations on $\mathrm{KI}$ transect in the lower Suwannee River floodplain, Florida

\begin{tabular}{ccccc}
\hline $\begin{array}{c}\text { Marker } \\
\text { number }\end{array} \begin{array}{c}\text { Distance } \\
\text { from } \\
\text { beginning } \\
\text { of transect }\end{array}$ & $\begin{array}{c}\text { Distance } \\
\text { from } \\
\text { previous } \\
\text { marker }\end{array}$ & Latitude & Longitude \\
\hline 122 & 0.0 & - & $29^{\circ} 26^{\prime} 21.6^{\prime \prime}$ & $83^{\circ} 01^{\prime} 10.5^{\prime \prime}$ \\
123 & 36.9 & 36.9 & $29^{\circ} 26^{\prime} 22.6^{\prime \prime}$ & $83^{\circ} 01^{\prime} 09.8^{\prime \prime}$ \\
124 & 74.0 & 37.1 & $29^{\circ} 26^{\prime} 23.6^{\prime \prime}$ & $83^{\circ} 01^{\prime} 09.1^{\prime \prime}$ \\
125 & 100.0 & 26.0 & $29^{\circ} 26^{\prime} 24.4^{\prime \prime}$ & $83^{\circ} 01^{\prime} 08.6^{\prime \prime}$ \\
\hline
\end{tabular}




\section{Turkey Island (TK)}

TK transect is on LSNWR lands. A locked gate restricts access to the road leading to this transect. Contact the Refuge Manager at (352) 493-0238 to make arrangements to access this property. The location of the LSNWR Headquarters, approximately 2 miles south of Fowlers Bluff on Highway 347 , is shown on the map in figure 19.

TK transect is located in western Levy County in the LSNWR, southeast of the Suwannee River. Public access roads in the refuge are highlighted in figure 19. The north entrance of the refuge is located 3.3 miles south of Fowlers Bluff, Florida, on County Road 347. To reach TK transect, turn west off of County Road 347 at the north entrance and follow this road 0.7 miles to a $90^{\circ}$ turn to the left, then immediately look for a road on the right. Follow this road to the second sharp $90^{\circ}$ turn to the left (fig. 20).

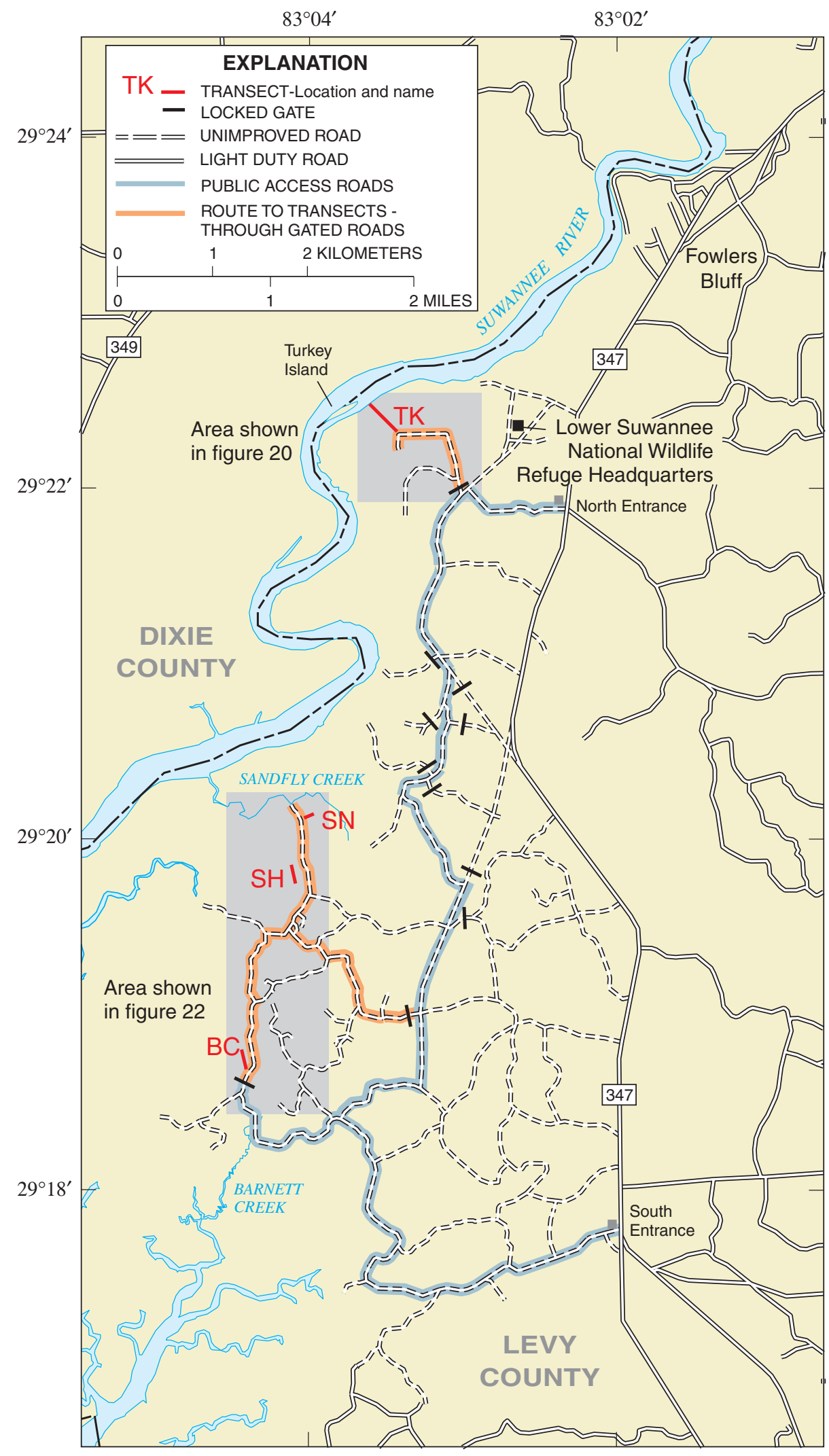

Figure 19. $\mathrm{TK}, \mathrm{SN}, \mathrm{SH}$, and $\mathrm{BC}$ transect locations with surrounding area in the lower Suwannee River floodplain, Florida. 


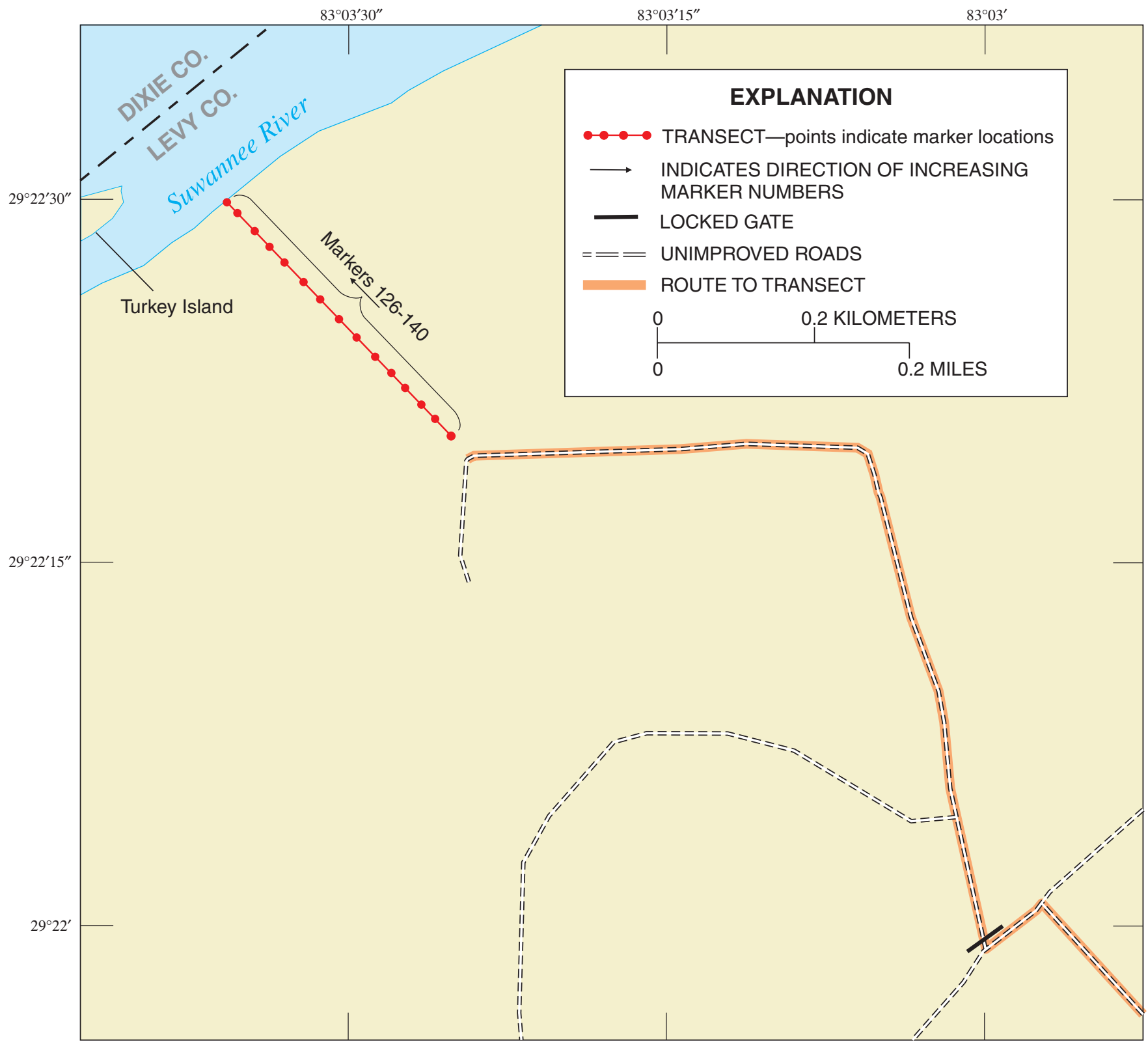

Figure 20. TK transect with marker locations and access roads in the lower Suwannee River floodplain, Florida. 
TK is a 411.9-meter transect with a compass bearing of $320^{\circ}$ from marker 126. Latitude and longitude of each marker on the transect are listed in table 8 .

Land-surface elevations of the transect line are provided in figure 21 . The peaks that appear on the transect are hummocks, mounds around the bases of trees elevated above the surrounding ground. Hummocks are present at nearly all transects but are most commonly exist on those in the lower tidal portion of the river. The lowest elevation on this transect is a tidal creek, located between markers 135 and 136, approximately 290 meters from the beginning of the transect.

Table 8. Marker locations on TK transect in the lower Suwannee River floodplain, Florida

\begin{tabular}{ccccc}
\hline $\begin{array}{c}\text { Marker } \\
\text { number }\end{array}$ & $\begin{array}{c}\text { Distance from } \\
\text { beginning of } \\
\text { transect }\end{array}$ & $\begin{array}{c}\text { Distance from } \\
\text { previous marker }\end{array}$ & Latitude & Longitude \\
\hline 126 & 0.0 & - & $29^{\circ} 22^{\prime} 19.4^{\prime \prime}$ & $83^{\circ} 03^{\prime} 25.7^{\prime \prime}$ \\
127 & 29.6 & 29.6 & $29^{\circ} 22^{\prime} 20.1^{\prime \prime}$ & $83^{\circ} 03^{\prime} 26.5^{\prime \prime}$ \\
128 & 54.6 & 25.0 & $29^{\circ} 22^{\prime} 20.6^{\prime \prime}$ & $83^{\circ} 03^{\prime} 27.1^{\prime \prime}$ \\
129 & 84.3 & 29.7 & $29^{\circ} 22^{\prime} 21.3^{\prime \prime}$ & $83^{\circ} 03^{\prime} 27.9^{\prime \prime}$ \\
130 & 110.5 & 26.2 & $29^{\circ} 22^{\prime} 22.0^{\prime \prime}$ & $83^{\circ} 03^{\prime} 28.5^{\prime \prime}$ \\
131 & 140.4 & 29.9 & $29^{\circ} 22^{\prime} 22.7^{\prime \prime}$ & $83^{\circ} 03^{\prime} 29.3^{\prime \prime}$ \\
132 & 173.1 & 32.7 & $29^{\circ} 22^{\prime} 23.4^{\prime \prime}$ & $83^{\circ} 03^{\prime} 30.1^{\prime \prime}$ \\
133 & 205.9 & 32.8 & $29^{\circ} 22^{\prime} 24.2^{\prime \prime}$ & $83^{\circ} 03^{\prime} 31.0^{\prime \prime}$ \\
134 & 241.4 & 35.5 & $29^{\circ} 22^{\prime} 25.0^{\prime \prime}$ & $83^{\circ} 03^{\prime} 31.9^{\prime \prime}$ \\
135 & 271.0 & 29.6 & $29^{\circ} 22^{\prime} 25.7^{\prime \prime}$ & $83^{\circ} 03^{\prime} 32.7^{\prime \prime}$ \\
136 & 305.8 & 34.8 & $29^{\circ} 22^{\prime} 26.5^{\prime \prime}$ & $83^{\circ} 03^{\prime} 33.6^{\prime \prime}$ \\
137 & 333.0 & 27.2 & $29^{\circ} 22^{\prime} 27.2^{\prime \prime}$ & $83^{\circ} 03^{\prime} 34.3^{\prime \prime}$ \\
138 & 361.1 & 28.1 & $29^{\circ} 22^{\prime} 27.8^{\prime \prime}$ & $83^{\circ} 03^{\prime} 35.0^{\prime \prime}$ \\
139 & 392.2 & 31.1 & $29^{\circ} 22^{\prime} 28.6^{\prime \prime}$ & $83^{\circ} 03^{\prime} 35.8^{\prime \prime}$ \\
140 & 411.9 & 19.7 & $29^{\circ} 22^{\prime} 29.0^{\prime \prime}$ & $83^{\circ} 03^{\prime} 36.3^{\prime \prime}$ \\
\hline
\end{tabular}

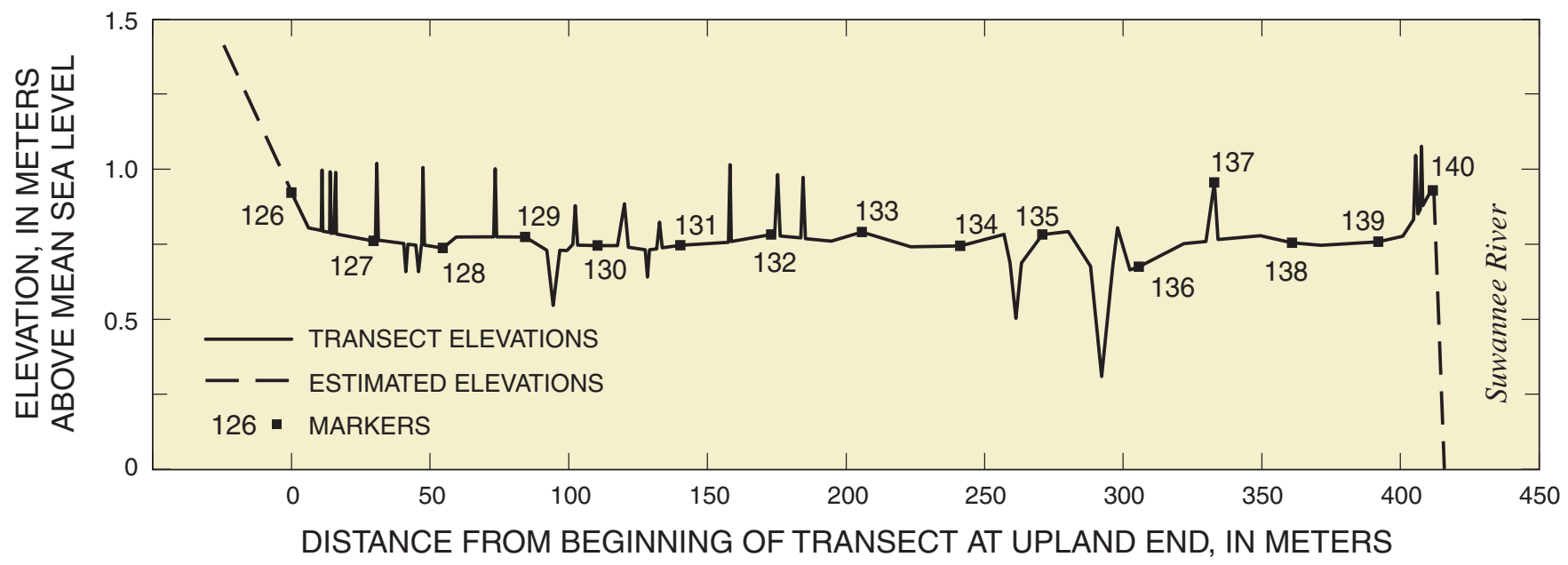

Figure 21. Land-surface elevations and marker locations at TK transect in the lower Suwannee River floodplain, Florida. 


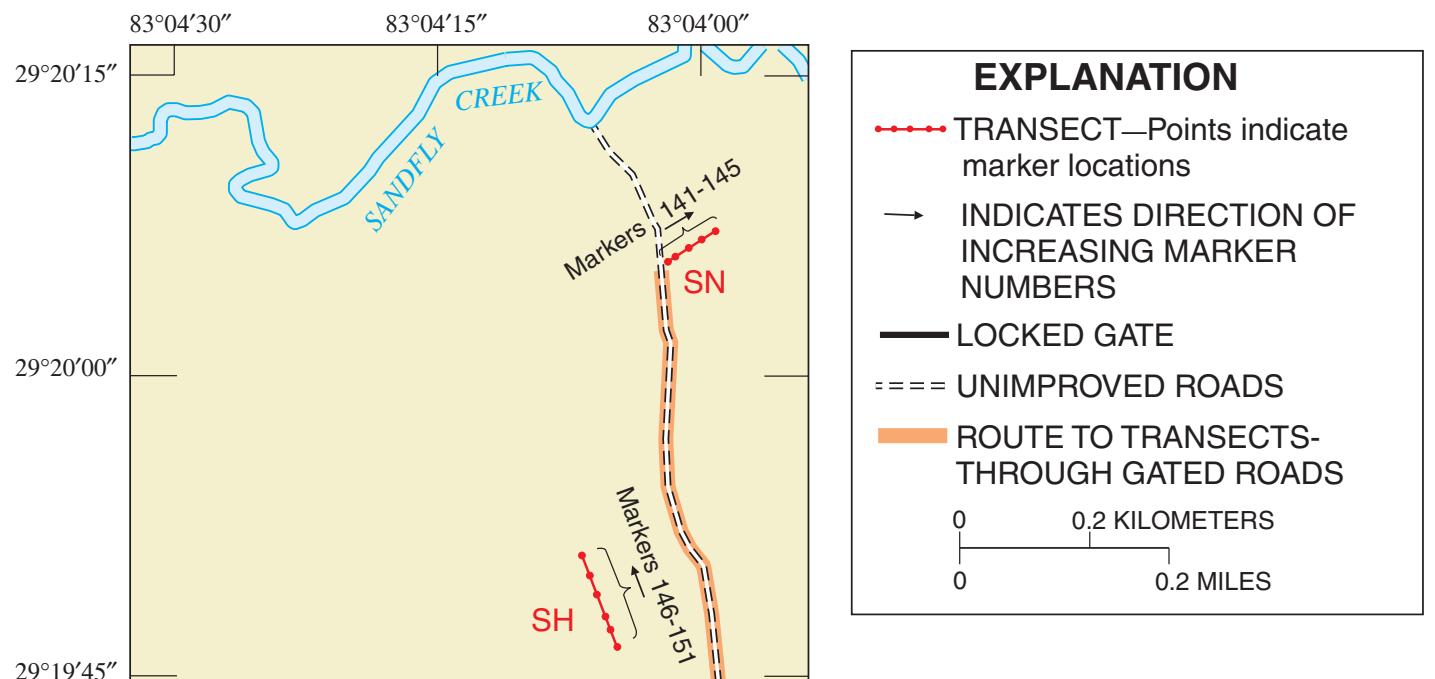

\section{Sandfly North (SN)}

SN transect is located in the LSNWR. Road access to this transect is restricted by a locked gate. Site visits to this transect will require the permission of the Refuge Manager, LSNWR Headquarters Office (fig. 19), at (352) 493-0238.

$\mathrm{SN}$ transect is located in western Levy County, Florida, approximately 0.5 mile southeast of the Suwannee River. The transect can be reached from either the north or south entrance to the LSNWR (fig. 19). The north entrance is located 3.3 miles south of Fowlers Bluff, Florida, on County Road 347. To reach the transect from this entrance follow the public access road 4.7 miles and look for a road on the right with a locked gate. After passing through this gate, go 0.9 miles down this road to a fork, take the right fork and then follow this road 0.3 miles to a T-intersection. Turn right and follow this road 0.8 miles to the transect. $\mathrm{SN}$ transect is located on the east side of the road (fig. 22).

$\mathrm{SN}$ transect is 88.3 meters long and has a compass bearing of $58^{\circ}$ from marker 141 . The geographic location of each marker on this transect is listed in table 9.

Land-surface elevations along the transect are presented in figure 23. The lowest elevation on the transect line is in a tidal creek, located approximately 30 meters from the beginning of the transect. Several hummocks were also surveyed along this transect. 
Table 9. Marker locations on SN transect in the lower Suwannee River floodplain, Florida

\begin{tabular}{ccccc}
\hline $\begin{array}{c}\text { Marker } \\
\text { number }\end{array}$ & $\begin{array}{c}\text { Distance from } \\
\text { beginning of } \\
\text { transect }\end{array}$ & $\begin{array}{c}\text { Distance from } \\
\text { previous marker }\end{array}$ & Latitude & Longitude \\
\hline 141 & 0.0 & - & $29^{\circ} 20^{\prime} 04.8^{\prime \prime}$ & $83^{\circ} 04^{\prime} 02.4^{\prime \prime}$ \\
142 & 15.3 & 15.3 & $29^{\circ} 20^{\prime} 05.1^{\prime \prime}$ & $83^{\circ} 04^{\prime} 02.0^{\prime \prime}$ \\
143 & 38.6 & 23.3 & $29^{\circ} 20^{\prime} 05.5^{\prime \prime}$ & $83^{\circ} 04^{\prime} 01.2^{\prime \prime}$ \\
144 & 62.7 & 24.1 & $29^{\circ} 20^{\prime} 06.0^{\prime \prime}$ & $83^{\circ} 04^{\prime} 00.5^{\prime \prime}$ \\
145 & 88.3 & 25.6 & $29^{\circ} 20^{\prime} 06.4^{\prime \prime}$ & $83^{\circ} 04^{\prime} 59.7^{\prime \prime}$ \\
\hline
\end{tabular}

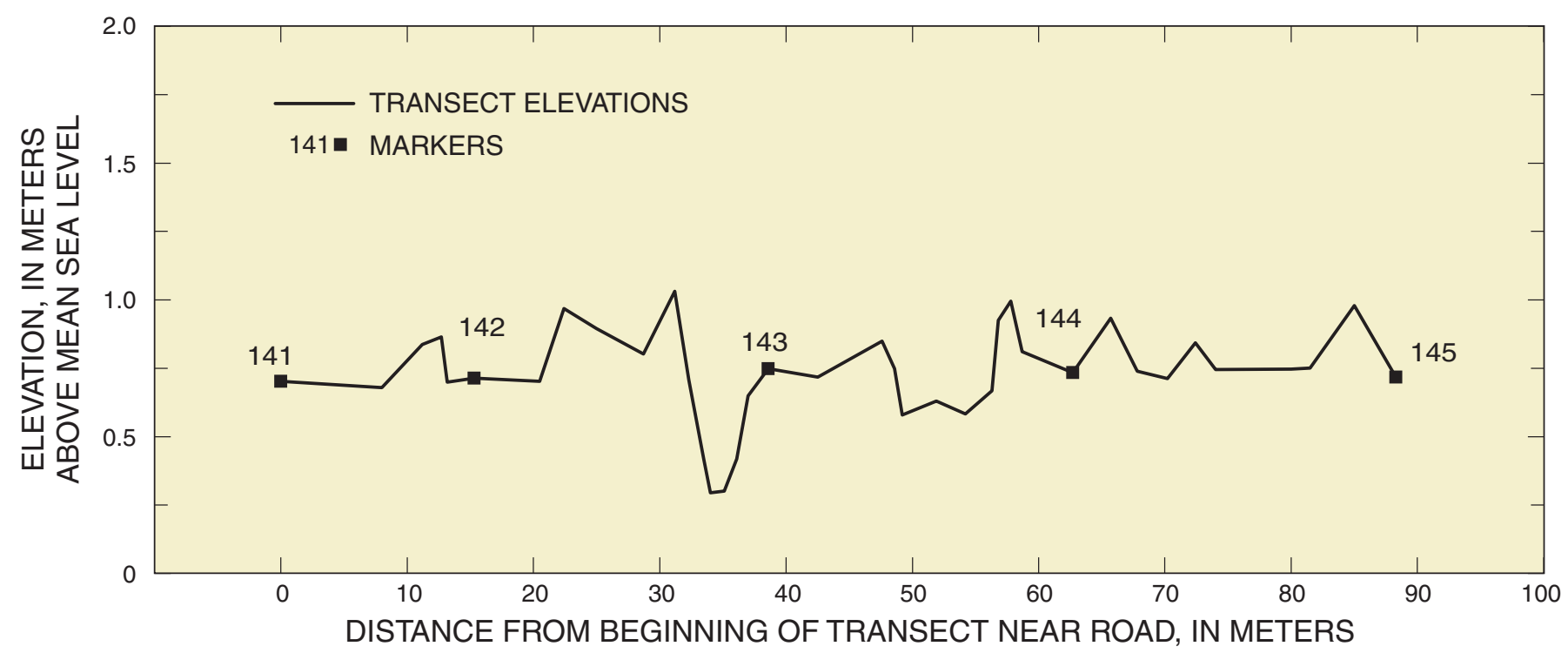

Figure 23. Land-surface elevations and marker locations at SN transect in the lower Suwannee River floodplain, Florida. 


\section{Sandfly Hammock (SH)}

$\mathrm{SH}$ transect is located in the is located approximately 3.3 miles LSNWR. A locked gate restricts access to the roads to this transect. Access to this site will require permission from the LSNWR Refuge Manager, LSNWR Headquarters Office (fig. 19), at (352) 493-0238.

$\mathrm{SH}$ transect is located in western Levy County, Florida, approximately 0.8 mile southeast of the Suwannee River. The transect can be reached from either the north or south entrance to the refuge (fig. 19). The north entrance south of Fowlers Bluff, Florida, on County Road 347 . To reach the transect from this entrance follow the public access road 4.7 miles and look for a road on the right with a locked gate. After passing through this gate, go 0.9 miles down this road to a fork, take the right fork and then follow this road 0.3 miles to a T-intersection. Turn right and follow this road 0.3 miles to the transect. This transect is not visible from the road. SH is aligned parallel to the access road and is located approximately 0.1 mile west of the road (fig. 22).

SH transect is 151 meters in length and has a compass bearing of $340^{\circ}$ starting at marker 146 . The latitude and longitude of each marker on the transect line are listed in table 10.

\section{A cross-section of the} transect line illustrates the land-surface elevations and marker locations along SH (fig. 24). The estimated elevations connect the transect to a nearby pond.

Table 10. Marker locations on SH transect in the lower Suwannee River floodplain, Florida

\begin{tabular}{ccccc}
\hline $\begin{array}{c}\text { Marker } \\
\text { number }\end{array}$ & $\begin{array}{c}\text { Distance from } \\
\text { beginning of } \\
\text { transect }\end{array}$ & $\begin{array}{c}\text { Distance from } \\
\text { previous marker }\end{array}$ & Latitude & Longitude \\
\hline 146 & 0.0 & - & $29^{\circ} 19^{\prime} 45.6^{\prime \prime}$ & $83^{\circ} 04^{\prime} 05.3^{\prime \prime}$ \\
147 & 28.6 & 28.6 & $29^{\circ} 19^{\prime} 46.5^{\prime \prime}$ & $83^{\circ} 04^{\prime} 05.7^{\prime \prime}$ \\
148 & 50.0 & 21.4 & $29^{\circ} 19^{\prime} 47.1^{\prime \prime}$ & $83^{\circ} 04^{\prime} 06.0^{\prime \prime}$ \\
149 & 86.0 & 36.0 & $29^{\circ} 19^{\prime} 48.2^{\prime \prime}$ & $83^{\circ} 04^{\prime} 06.4^{\prime \prime}$ \\
150 & 117.4 & 31.4 & $29^{\circ} 19^{\prime} 49.2^{\prime \prime}$ & $83^{\circ} 04^{\prime} 06.9^{\prime \prime}$ \\
151 & 151.0 & 33.6 & $29^{\circ} 19^{\prime} 50.2^{\prime \prime}$ & $83^{\circ} 04^{\prime} 07.3^{\prime \prime}$ \\
\hline
\end{tabular}

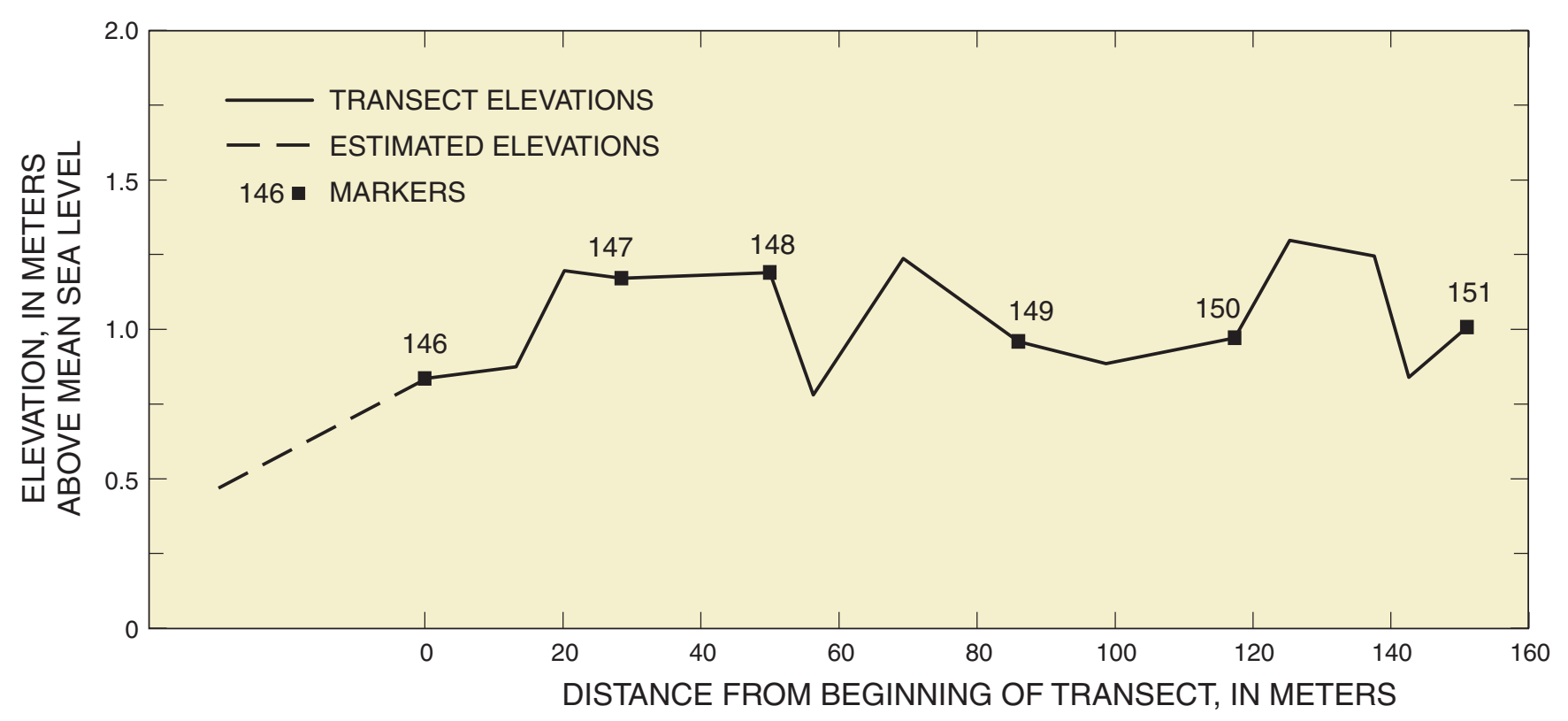

Figure 24. Land-surface elevations and marker locations at SH transect in the lower Suwannee River floodplain, Florida. 


\section{Barnett Creek (BC)}

$\mathrm{BC}$ transect is located on LSNWR lands. The access road to $\mathrm{BC}$ transect is restricted by a locked gate. Contact the LSNWR Manager, Headquarters Office (fig. 19), at (352) 493-0238 for permission to access this area.

$\mathrm{BC}$ transect is located in western Levy County. The shortest route to the transect is through the south entrance of the LSNWR, but it is also accessible from the north entrance (fig. 19). The south entrance is located 7.8 miles south of Fowlers Bluff, Florida, on County Road 347. Follow the public access road from this entrance approximately 4.3 miles to a locked gate. After passing through this gate, follow this road 0.2 miles to the transect. BC transect is located on the west side of the road (fig. 22).
$\mathrm{BC}$ transect is 215.6 meters long and has a compass bearing of $350^{\circ}$ from marker 152 to $156 ; 344^{\circ}$ from marker 156 to 157 , and $320^{\circ}$ from marker 157 to 159 . The latitude and longitude of each marker are listed in table 11.

The surveyed elevations of the $\mathrm{BC}$ transect line are presented in figure 25 . The estimated elevations near marker 152 represent a manmade roadside ditch.

Table 11. Marker locations on BC transect in lower Suwannee River Floodplain, Florida

\begin{tabular}{ccccc}
\hline $\begin{array}{c}\text { Marker } \\
\text { number }\end{array}$ & $\begin{array}{c}\text { Distance from } \\
\text { beginning of } \\
\text { transect }\end{array}$ & $\begin{array}{c}\text { Distance from } \\
\text { previous marker }\end{array}$ & Latitude & Longitude \\
\hline 152 & 0.0 & - & $29^{\circ} 18^{\prime} 41.0^{\prime \prime}$ & $83^{\circ} 04^{\prime} 23.0^{\prime \prime}$ \\
153 & 23.8 & 23.8 & $29^{\circ} 18^{\prime} 41.7^{\prime \prime}$ & $83^{\circ} 04^{\prime} 23.2^{\prime \prime}$ \\
154 & 44.8 & 21.0 & $29^{\circ} 18^{\prime} 42.4^{\prime \prime}$ & $83^{\circ} 04^{\prime} 23.5^{\prime \prime}$ \\
155 & 73.0 & 28.2 & $29^{\circ} 18^{\prime} 43.2^{\prime \prime}$ & $83^{\circ} 04^{\prime} 23.8^{\prime \prime}$ \\
156 & 103.8 & 30.8 & $29^{\circ} 18^{\prime} 44.2^{\prime \prime}$ & $83^{\circ} 04^{\prime} 24.1^{\prime \prime}$ \\
157 & 147.3 & 43.5 & $29^{\circ} 18^{\prime} 45.5^{\prime \prime}$ & $83^{\circ} 04^{\prime} 24.8^{\prime \prime}$ \\
158 & 189.1 & 41.8 & $29^{\circ} 18^{\prime} 46.4^{\prime \prime}$ & $83^{\circ} 04^{\prime} 26.0^{\prime \prime}$ \\
159 & 215.6 & 26.5 & $29^{\circ} 18^{\prime} 47.0^{\prime \prime}$ & $83^{\circ} 04^{\prime} 26.7^{\prime \prime}$ \\
\hline
\end{tabular}

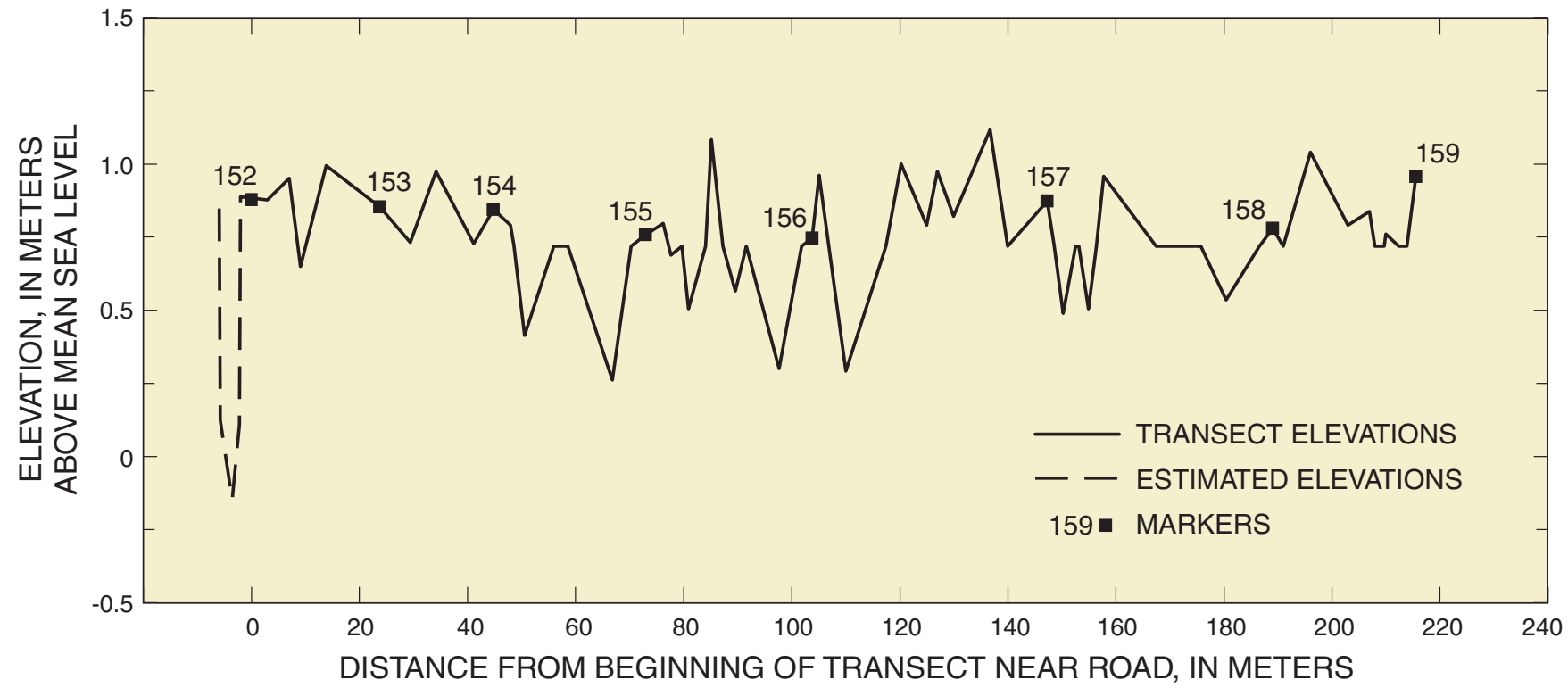

Figure 25. Land-surface elevations and marker locations at BC transect in the lower Suwannee River floodplain, Florida. 


\section{Lock (LK)}

LK transect is located on LSNWR lands. Contact the Refuge Manager, LSNWR Headquarters Office (fig. 19), at (352) 4930238 for permission to access this area.
LK is located near the town of Suwannee, Florida, in southern Dixie County. This transect is only accessible by river and there are several boat ramps in Suwannee, Florida, (fig. 26) that can be used as launch sites. LK transect is located roughly halfway between Lock Creek and Demory Creek (fig. 27). A Global Positioning System (GPS) unit is needed to locate this site.

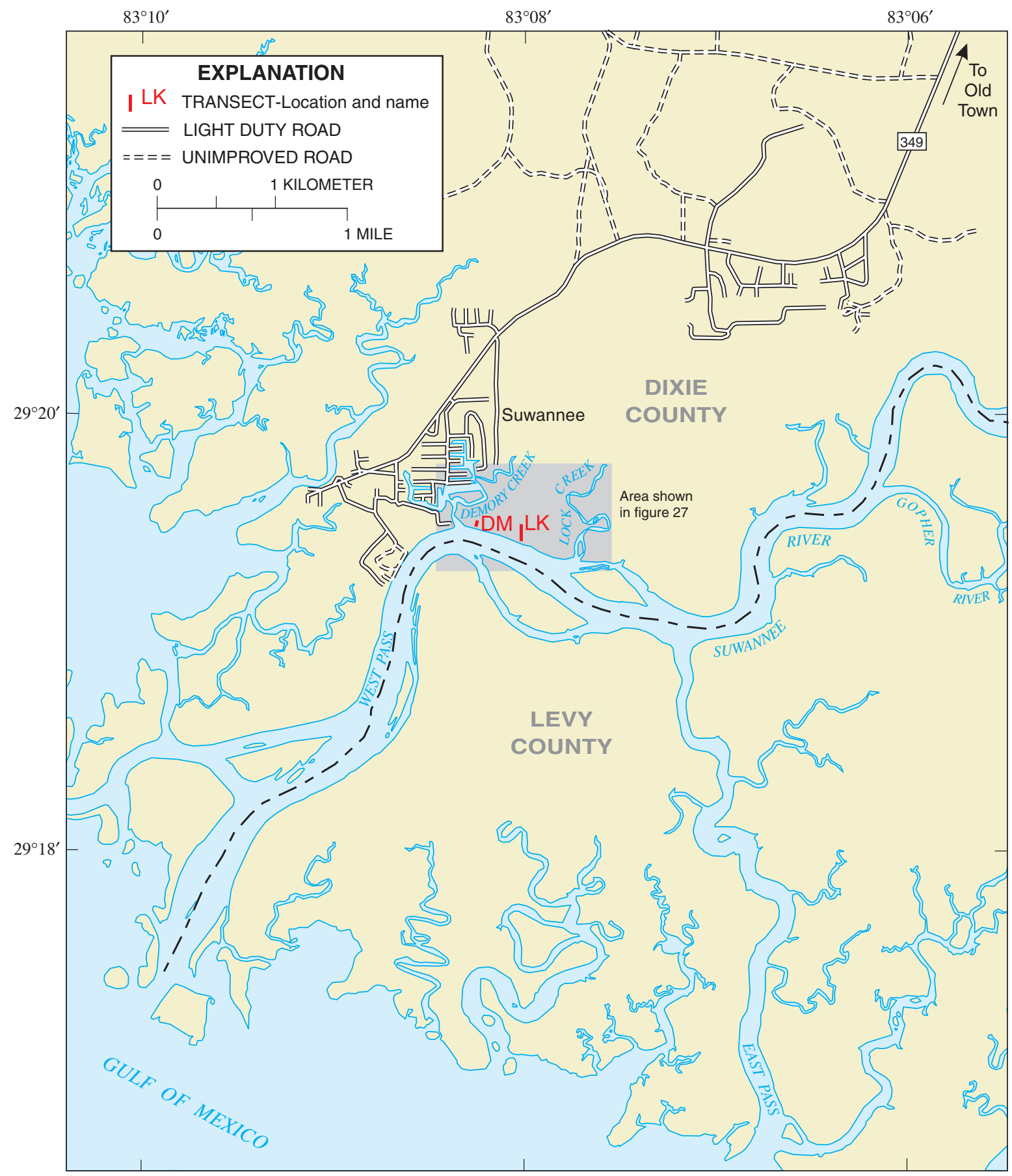

Figure 26. LK and DM transect locations and surrounding area in the lower Suwannee River floodplain, Florida. 

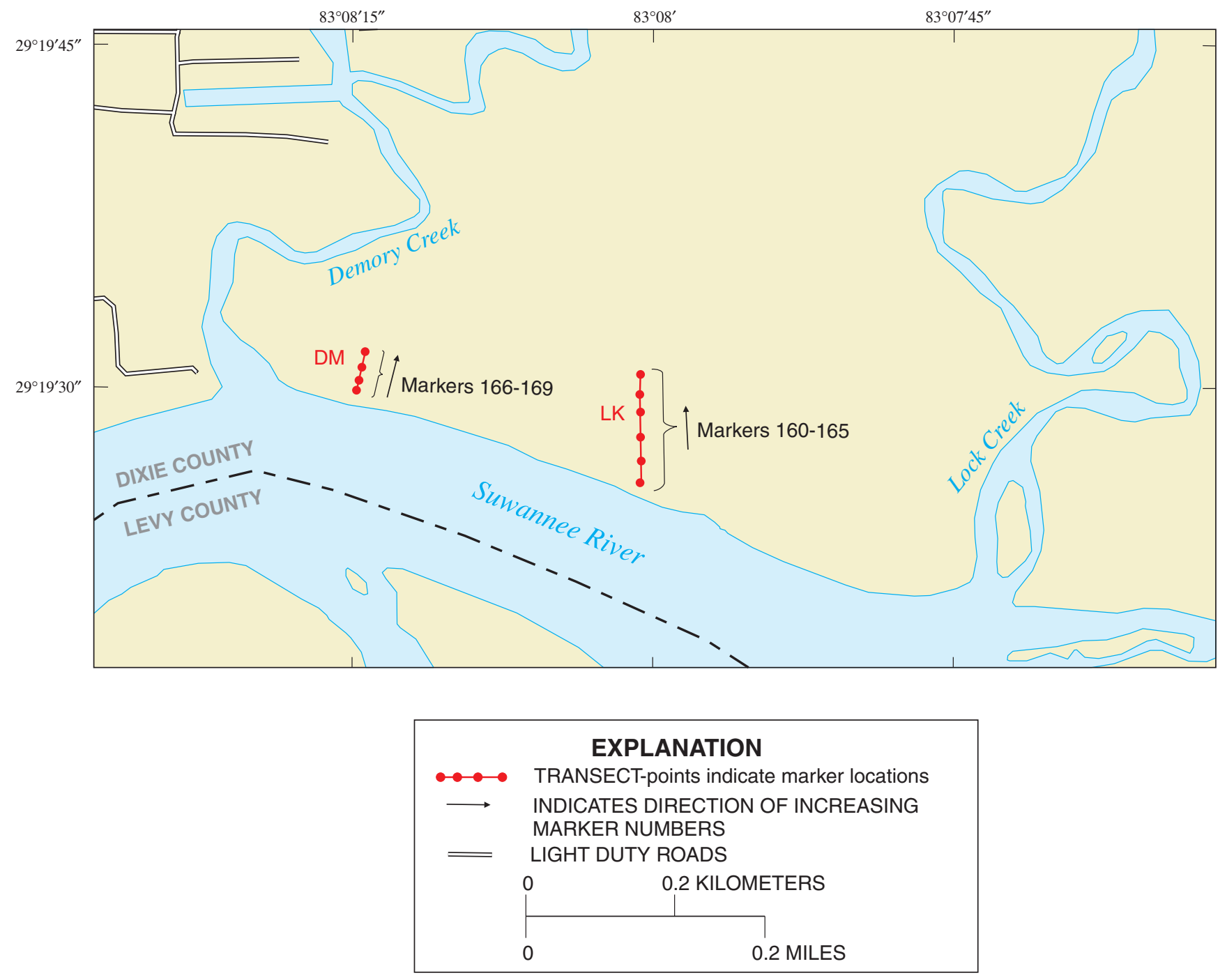

Figure 27. LK and DM transects with marker locations and access roads in the lower Suwannee River floodplain, Florida. 
LK transect is 145.5 meters in length and has a compass bearing of $0^{\circ}$ from markers 160 to 164 , then changes course slightly to $3^{\circ}$ between markers 164 and 165. The latitude and longitude of each marker are presented in table 12.

A cross-section of the transect showing land-surface elevations (fig. 28) illustrates the hummock and mud floor microtopography at this transect. The mud floor of this transect is covered by the high tide almost every day, but the tops of the hummocks are covered only during storm surges or major floods (fig. 16, Light and others, in press).

Table 12. Marker locations on LK transect in the lower Suwannee River floodplain, Florida

\begin{tabular}{ccccc}
\hline $\begin{array}{c}\text { Marker } \\
\text { number }\end{array}$ & $\begin{array}{c}\text { Distance from } \\
\text { beginning of } \\
\text { transect }\end{array}$ & $\begin{array}{c}\text { Distance from } \\
\text { previous } \\
\text { marker }\end{array}$ & Latitude & Longitude \\
\hline 160 & 0.0 & - & $29^{\circ} 19^{\prime} 25.0^{\prime \prime}$ & $83^{\circ} 08^{\prime} 01.1^{\prime \prime}$ \\
161 & 28.7 & 28.7 & $29^{\circ} 19^{\prime} 26.0^{\prime \prime}$ & $83^{\circ} 08^{\prime} 01.1^{\prime \prime}$ \\
162 & 61.0 & 32.3 & $29^{\circ} 19^{\prime} 27.0^{\prime \prime}$ & $83^{\circ} 08^{\prime} 01.2^{\prime \prime}$ \\
163 & 93.5 & 32.5 & $29^{\circ} 19^{\prime} 28.1^{\prime \prime}$ & $83^{\circ} 08^{\prime} 01.2^{\prime \prime}$ \\
164 & 117.4 & 23.9 & $29^{\circ} 19^{\prime} 28.9^{\prime \prime}$ & $83^{\circ} 08^{\prime} 01.2^{\prime \prime}$ \\
165 & 145.5 & 28.1 & $29^{\circ} 19^{\prime} 29.7^{\prime \prime}$ & $83^{\circ} 08^{\prime} 01.2^{\prime \prime}$ \\
\hline
\end{tabular}

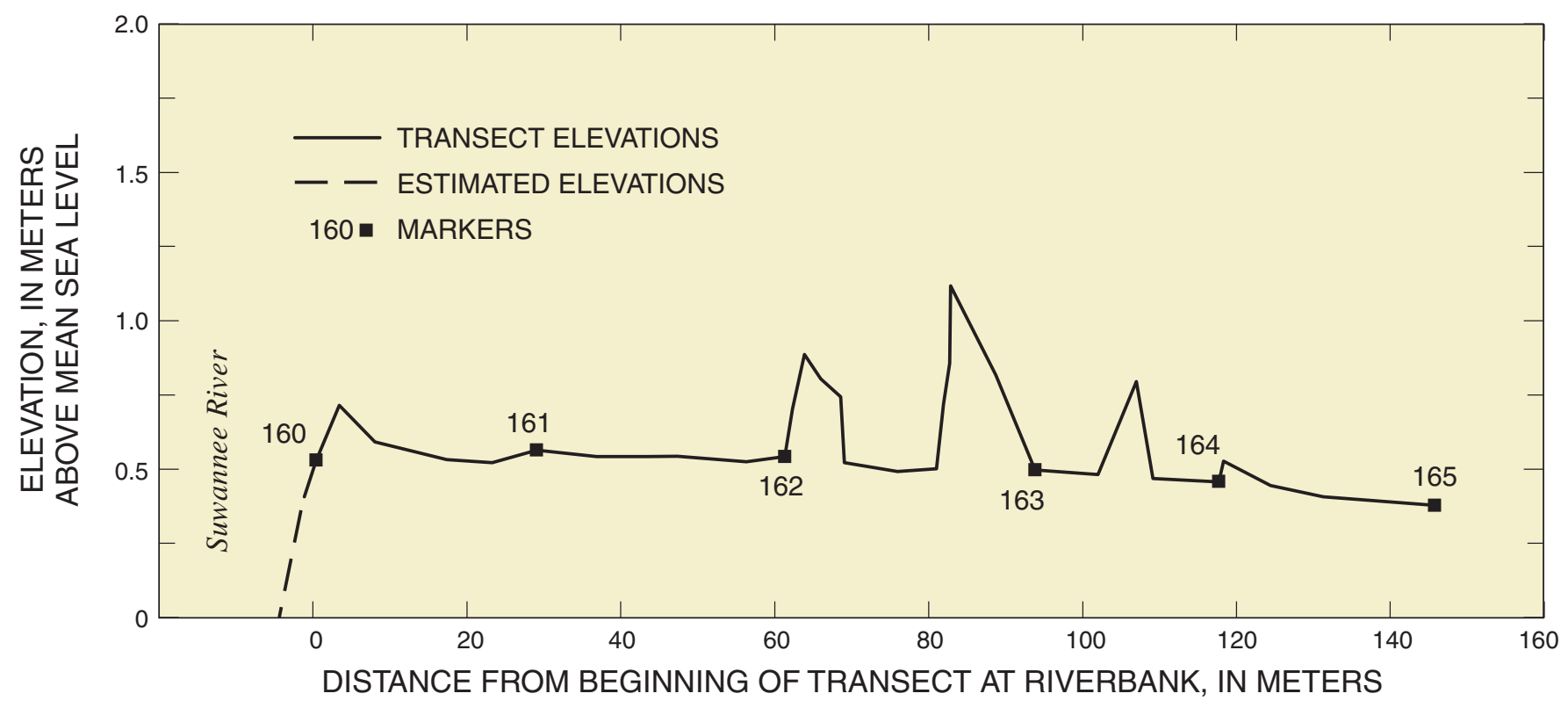

Figure 28. Land-surface elevations and marker locations at LK transect in the lower Suwannee River floodplain, Florida. 
DM is located on land that is below the mean high water line and assumed to belong to the State of Florida according to State policy in effect at the time the transect was established. If this policy changes in the future, the landowner will need to be contacted for permission prior to accessing this transect.

DM transect is located near the town of Suwannee, Florida, in southern Dixie County. The transect is only accessible by river and the nearest launch sites are located in the town of Suwannee, Florida (fig. 26). This transect is located near the mouth of Demory Creek (fig. 27). A GPS unit is required to locate the exact position of the transect along the river.

DM transect is 53.2 meters in length and is the shortest of the established transects. This transect line follows a compass bearing of $14^{\circ}$ from marker 166 . The latitude and longitude of each marker is provided in table 13.

DM transect has the lowest elevations and least topographic relief of all of the transects (fig. 29). There are no hummocks on this transect, but the mud floor contains many exposed gnarly roots.

Table 13. Marker locations on DM transect in the lower Suwannee River floodplain, Florida

\begin{tabular}{ccccc}
\hline $\begin{array}{c}\text { Marker } \\
\text { number }\end{array}$ & $\begin{array}{c}\text { Distance from } \\
\text { beginning of } \\
\text { transect }\end{array}$ & $\begin{array}{c}\text { Distance from } \\
\text { previous marker }\end{array}$ & Latitude & Longitude \\
\hline 166 & 0.0 & - & $29^{\circ} 19^{\prime} 29.0^{\prime \prime}$ & $83^{\circ} 08^{\prime} 15.3^{\prime \prime}$ \\
167 & 13.3 & 13.3 & $29^{\circ} 19^{\prime} 29.4^{\prime \prime}$ & $83^{\circ} 08^{\prime} 15.2^{\prime \prime}$ \\
168 & 31.3 & 18.0 & $29^{\circ} 19^{\prime} 30.0^{\prime \prime}$ & $83^{\circ} 08^{\prime} 15.1^{\prime \prime}$ \\
169 & 53.2 & 21.9 & $29^{\circ} 19^{\prime} 30.7^{\prime \prime}$ & $83^{\circ} 08^{\prime} 14.9^{\prime \prime}$ \\
\hline
\end{tabular}

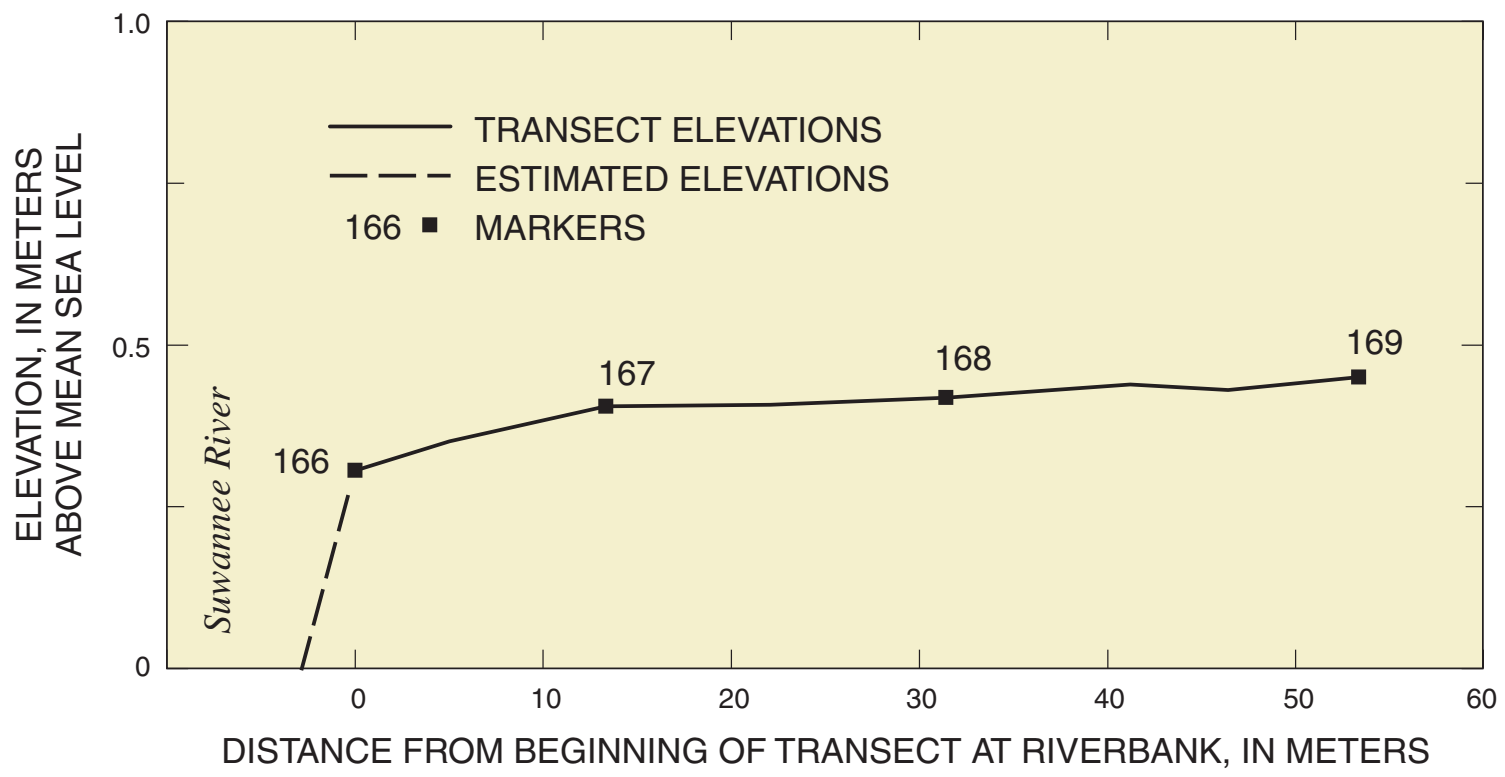

Figure 29. Land-surface elevations and marker locations at DM transect in the lower Suwannee River floodplain, Florida. 


\section{REFERENCES}

Clewell, A.F., 1985, Guide to the vascular plants of the Florida Panhandle: Tallahassee, Florida State University Press, 605 p.

Darst, M.R., Light, H. M., and Lewis, L.J., 2002, Ground cover vegetation in wetland forests in the Lower Suwannee River floodplain, Florida, and potential impacts of flow reductions: U.S. Geological Survey Water-

Resources Investigations Report 02-4027.

Godfrey, R.K., 1988, Trees, shrubs, and woody vines of northern Florida and adjacent Georgia and Alabama: Athens, The University of Georgia Press, 734 p.
Light, H.M., Darst, M.R., Lewis, L.J., and Howell, D.A., in press, Hydrology, vegetation, and soils of riverine and tidal floodplain forests of the lower Suwannee River, Florida, and potential impacts of flow reductions: U.S. Geological Survey Professional Paper 1656A. 


\section{Appendixes}


Appendix 1. Canopy tree species and measurements and marker locations on transects in the lower Suwannee River floodplain, Florida

\section{This appendix contains the following sections:}

$\begin{array}{ll}\text { A } & \text { CF transect } \\ \text { B } & \text { LL transect } \\ \text { C } & \text { FK transect } \\ \text { D } & \text { MS transect } \\ \text { E } & \text { KN transect } \\ \text { F } & \text { KI transect } \\ \text { G } & \text { TK transect } \\ \text { H } & \text { SN transect } \\ \text { I } & \text { SH transect } \\ \text { J } & \text { BC transect } \\ \text { K } & \text { LK transect } \\ \text { L } & \text { DM transect }\end{array}$

[cm, centimeters; $\mathrm{m}$, meters; dbh, diameter at breast height; separate measurements for dbh are shown for trunks of multiple trunked trees greater than $4 \mathrm{~cm}$ ]

List of tree species codes with scientific names and common name equivalents

[Plant nomenclature used in this report follows that by Godfrey (1988) unless otherwise indicated]

\begin{tabular}{|c|c|c|}
\hline Code & $\underline{\text { Scientific Name }}$ & $\underline{\text { Common name }}$ \\
\hline acerub & Acer rubrum $\mathrm{L}$. & red maple \\
\hline betnig & Betula nigra $\mathrm{L}$. & river birch \\
\hline carcar & Carpinus caroliniana Walt. & ironwood \\
\hline caraqu & Carya aquatica (Michx. f.) Nutt. & water hickory \\
\hline cargla & Carya glabra (Mill.) Sweet & pignut hickory \\
\hline cellae & Celtis laevigata Nutt. & hackberry \\
\hline cepocc & Cephalanthus occidentalis L. & buttonbush \\
\hline corfoe & Cornus foemina Mill. & stiffcornel dogwood \\
\hline crafla & Crataegus flava Ait. & yellow haw \\
\hline cravir & Crataegus viridis $\mathrm{L}$. & green haw \\
\hline cyrrac & Cyrilla racemiflora $\mathrm{L}$. & titi \\
\hline diovir & Diospyros virginiana $\mathrm{L}$. & persimmon \\
\hline foracu & Forestiera acuminata (Michx.) Poir. in Lam. & swamp-privet \\
\hline fracar & Fraxinus caroliniana Mill. & pop ash \\
\hline frapro & Fraxinus profunda (Bush) Bush & pumpkin ash \\
\hline gleaqu & Gleditsia aquatica Marsh. & water locust \\
\hline ilecas & Ilex cassine $\mathrm{L}$. & dahoon \\
\hline iledec & Ilex decidua Walt. var. curtissii Fern. & possum-haw \\
\hline ileopa & Ilex opaca Ait. var. opaca & American holly \\
\hline junsil & Juniperus silicicola (Small) Bailey ${ }^{1}$ & southern red cedar \\
\hline liqsty & Liquidambar styraciflua $\mathrm{L}$. & sweetgum \\
\hline magvir & Magnolia virginiana $\mathrm{L}$. & sweetbay \\
\hline myrcer & Myrica cerifera $\mathrm{L}$. & wax-myrtle \\
\hline nysaqu & Nyssa aquatica $\mathrm{L}$. & water tupelo \\
\hline nysbif & Nyssa biflora Walt. ${ }^{1}$ & swamp gum \\
\hline nyssyl & Nyssa sylvatica Marsh..$^{1}$ & blackgum \\
\hline ostvir & Ostrya virginiana (Mill.) K. Koch & eastern hophornbeam \\
\hline perpal & Persea palustris (Raf.) Sarg. & swamp red bay \\
\hline pinell & Pinus elliottii Engelm. var. elliottii & slash pine \\
\hline pingla & Pinus glabra Walt. & spruce pine \\
\hline pintae & Pinus taeda $\mathrm{L}$. & planer-tree \\
\hline plaaqu & Planera aquatica J. F. Gmel. & loblolly pine \\
\hline
\end{tabular}


Appendix 1. Canopy tree species and measurements and marker locations on transects in the lower Suwannee River floodplain, Florida (Continued)

$\begin{array}{lll}\text { quegem } & \text { Quercus geminata } \text { Small } & \text { sand live oak } \\ \text { quehem } & \text { Quercus hemisphaerica } \text { Bartr. ex Willd. } & \text { laurel oak } \\ \text { quelau } & \text { Quercus laurifolia } \text { Michx. } & \text { swamp laurel oak } \\ \text { quelyr } & \text { Quercus lyrata Walt. } & \text { overcup oak } \\ \text { quenig } & \text { Quercus nigra } \text { L. } & \text { water oak } \\ \text { quevir } & \text { Quercus virginiana } \text { Mill. } & \text { live oak } \\ \text { sabpal } & \text { Sabal palmetto Lodd. ex J. S. Shult. \& J. H. Shult. } & \text { cabbage palm } \\ \text { salcar } & \text { Salix caroliniana Michx. } & \text { Carolina willow } \\ \text { salnig } & \text { Salix nigra } \text { L. } & \text { black willow } \\ \text { taxdis } & \text { Taxodium distichum } \text { (L.) L. C. Rich. } & \text { bald cypress } \\ \text { ulmala } & \text { Ulmus alata } \text { Michx. } & \text { winged elm } \\ \text { ulmame } & \text { Ulmus americana } \text { L. } & \text { American elm } \\ \text { ulmcra } & \text { Ulmus } \text { crassifolia } \text { Nutt. } & \text { cedar elm } \\ \text { vacarb } & \text { Vaccinium arboreum Marsh. } & \text { sparkleberry } \\ \text { vibobo } & \text { Viburnum } \text { obovatum } \text { Walt. } & \text { small viburnum } \\ \text { vitcin } & \text { Vitis cinerea } \text { (Engelm. ex Gray) Millardet } & \text { downy winter grape }\end{array}$

${ }^{1}$ Clewell (1985). 
A CF: The sampling width of this transect is 10 meters, 5 meters on either side of the transect line

\begin{tabular}{|c|c|c|c|}
\hline $\begin{array}{l}\text { Distance from } \\
\text { beginning of } \\
\text { segment, in } \mathrm{m}\end{array}$ & $\begin{array}{c}\text { Tree } \\
\text { species }\end{array}$ & $\begin{array}{l}\text { Dbh, } \\
\text { in cm }\end{array}$ & Side \\
\hline \multicolumn{4}{|c|}{ West Segment } \\
\hline 0.0 & \multicolumn{3}{|c|}{ marker \# 1} \\
\hline \multirow{7}{*}{1.3} & \multirow{7}{*}{ acerub } & 6.6 & \multirow{7}{*}{$\mathrm{n}$} \\
\hline & & 8.6 & \\
\hline & & 9.8 & \\
\hline & & 10.2 & \\
\hline & & 12.3 & \\
\hline & & 11.3 & \\
\hline & & 11.7 & \\
\hline 1.5 & taxdis & 39.4 & $\mathrm{n}$ \\
\hline \multirow{2}{*}{4.7} & \multirow{2}{*}{ fracar } & 13.2 & \multirow[b]{2}{*}{$\mathrm{s}$} \\
\hline & & 7.0 & \\
\hline 5.0 & betnig & 19.6 & $\mathrm{~s}$ \\
\hline 7.0 & taxdis & 52.3 & $\mathrm{~s}$ \\
\hline \multirow{2}{*}{8.8} & \multirow{2}{*}{ acerub } & 13.1 & \multirow{2}{*}{$\mathrm{s}$} \\
\hline & & 8.0 & \\
\hline 11.0 & taxdis & 43.0 & $\mathrm{~s}$ \\
\hline 11.2 & salcar & 19.2 & $\mathrm{~s}$ \\
\hline 11.2 & taxdis & 43.3 & $\mathrm{n}$ \\
\hline 11.7 & acerub & 16.0 & $\mathrm{n}$ \\
\hline 12.1 & plaaqu & 10.4 & $\mathrm{~s}$ \\
\hline 12.6 & plaaqu & 11.2 & $\mathrm{n}$ \\
\hline \multirow{2}{*}{13.0} & \multirow{2}{*}{ plaaqu } & 15.6 & \multirow{2}{*}{$\mathrm{s}$} \\
\hline & & 5.8 & \\
\hline 14.1 & fracar & 10.0 & $\mathrm{n}$ \\
\hline \multirow{3}{*}{14.8} & \multirow{3}{*}{ cepocc } & 10.1 & \multirow{3}{*}{$\mathrm{n}$} \\
\hline & & 6.1 & \\
\hline & & 9.5 & \\
\hline 16.1 & taxdis & 47.0 & $\mathrm{~s}$ \\
\hline 16.4 & cepocc & 11.5 & $\mathrm{~s}$ \\
\hline \multirow{5}{*}{18.9} & \multirow{5}{*}{ fracar } & 13.9 & \multirow{5}{*}{$\mathrm{n}$} \\
\hline & & 8.9 & \\
\hline & & 8.6 & \\
\hline & & 9.6 & \\
\hline & & 11.0 & \\
\hline 19.5 & taxdis & 35.9 & $\mathrm{~s}$ \\
\hline 20.1 & \multicolumn{3}{|c|}{ marker \# 2} \\
\hline 20.1 & taxdis & 34.9 & $\mathrm{~s}$ \\
\hline 21.5 & taxdis & 39.6 & $\mathrm{~s}$ \\
\hline 21.9 & cepocc & 12.3 & $\mathrm{~s}$ \\
\hline 26.5 & plaaqu & 15.3 & $\mathrm{n}$ \\
\hline \multirow{2}{*}{27.6} & \multirow{2}{*}{ plaaqu } & 10.1 & $\mathrm{n}$ \\
\hline & & 4.1 & \\
\hline 29.4 & plaaqu & 11.8 & $\mathrm{n}$ \\
\hline 29.5 & plaaqu & 11.2 & $\mathrm{n}$ \\
\hline 30.7 & plaaqu & 18.6 & $\mathrm{n}$ \\
\hline
\end{tabular}

\begin{tabular}{|c|c|c|c|}
\hline $\begin{array}{l}\text { Distance from } \\
\text { beginning of } \\
\text { segment in } \mathrm{m}\end{array}$ & $\begin{array}{c}\text { Tree } \\
\text { species }\end{array}$ & $\begin{array}{l}\text { Dbh, } \\
\text { in cm }\end{array}$ & Side \\
\hline 30.8 & plaaqu & 11.5 & $\mathrm{n}$ \\
\hline \multirow{2}{*}{32.9} & \multirow{2}{*}{ plaaqu } & 10.9 & \multirow{2}{*}{$\mathrm{s}$} \\
\hline & & 14.0 & \\
\hline \multirow{2}{*}{33.0} & \multirow{2}{*}{ fracar } & 11.9 & \multirow{2}{*}{$\mathrm{s}$} \\
\hline & & 6.6 & \\
\hline \multirow{2}{*}{34.3} & \multirow{2}{*}{ plaaqu } & 10.3 & \multirow{2}{*}{$\mathrm{s}$} \\
\hline & & 4.9 & \\
\hline 34.3 & taxdis & 38.2 & $\mathrm{n}$ \\
\hline \multirow{2}{*}{34.6} & \multirow{2}{*}{ plaaqu } & 11.2 & \multirow{2}{*}{$\mathrm{n}$} \\
\hline & & 9.0 & \\
\hline 35.2 & plaaqu & 15.1 & $\mathrm{~s}$ \\
\hline 35.8 & plaaqu & 15.5 & $\mathrm{n}$ \\
\hline 36.2 & taxdis & 49.6 & $\mathrm{~s}$ \\
\hline 37.4 & cepocc & 10.3 & $\mathrm{~s}$ \\
\hline 37.6 & plaaqu & 13.4 & $\mathrm{n}$ \\
\hline 39.8 & taxdis & 34.6 & $\mathrm{n}$ \\
\hline 40.1 & taxdis & 41.7 & $\mathrm{n}$ \\
\hline \multirow{3}{*}{40.7} & \multirow{3}{*}{ cepocc } & 10.0 & \multirow{3}{*}{$\mathrm{s}$} \\
\hline & & 7.0 & \\
\hline & & 6.7 & \\
\hline \multirow{2}{*}{41.6} & \multirow{2}{*}{ cepocc } & 10.5 & \multirow{2}{*}{$\mathrm{n}$} \\
\hline & & 6.4 & \\
\hline 41.6 & taxdis & 41.8 & $\mathrm{n}$ \\
\hline \multirow{6}{*}{42.1} & \multirow{6}{*}{ plaaqu } & 27.8 & \multirow{6}{*}{$\mathrm{s}$} \\
\hline & & 5.5 & \\
\hline & & 5.6 & \\
\hline & & 12.5 & \\
\hline & & 8.3 & \\
\hline & & 10.5 & \\
\hline 43.8 & gleaqu & 30.5 & $\mathrm{n}$ \\
\hline \multirow{2}{*}{44.4} & \multirow{2}{*}{ fracar } & 10.4 & \multirow{2}{*}{$\mathrm{s}$} \\
\hline & & 6.5 & \\
\hline 44.6 & & arker \# & \\
\hline 46.8 & taxdis & 32.0 & $\mathrm{n}$ \\
\hline 47.8 & gleaqu & 20.6 & $\mathrm{n}$ \\
\hline 178 & lo & 10.9 & \\
\hline $4 / .8$ & praaqu & 11.2 & S \\
\hline 48.2 & taxdis & 49.3 & $\mathrm{~s}$ \\
\hline 49.0 & taxdis & 37.1 & $\mathrm{n}$ \\
\hline 50.7 & taxdis & 36.6 & $\mathrm{n}$ \\
\hline 51.1 & betnig & 27.0 & $\mathrm{~s}$ \\
\hline 540 & fropr & 11.3 & \\
\hline 54.0 & fracar & 7.2 & $\mathrm{~s}$ \\
\hline 54.7 & gleaqu & 21.0 & $\mathrm{n}$ \\
\hline 57.2 & taxdis & 16.4 & $\mathrm{n}$ \\
\hline 59.0 & fracar & 13.1 & $\mathrm{~s}$ \\
\hline
\end{tabular}


A CF: The sampling width of this transect is 10 meters, 5 meters on either side of the transect line (Continued)

\begin{tabular}{|c|c|c|c|}
\hline $\begin{array}{l}\text { Distance from } \\
\text { beginning of } \\
\text { segment, in } \mathrm{m}\end{array}$ & $\begin{array}{c}\text { Tree } \\
\text { species }\end{array}$ & $\begin{array}{l}\text { Dbh, } \\
\text { in cm }\end{array}$ & Side \\
\hline 59.7 & серосc & 12.0 & $\mathrm{~s}$ \\
\hline 60.0 & fracar & 11.5 & $\mathrm{n}$ \\
\hline \multirow{4}{*}{62.6} & \multirow{4}{*}{ fracar } & 8.7 & $\mathrm{n}$ \\
\hline & & 12.4 & \multirow{3}{*}{$\mathrm{n}$} \\
\hline & & 10.5 & \\
\hline & & 9.6 & \\
\hline 63.7 & fracar & 11.3 & $\mathrm{n}$ \\
\hline 65.1 & fracar & 11.2 & $\mathrm{n}$ \\
\hline 65.1 & taxdis & 48.4 & $\mathrm{~s}$ \\
\hline 65.2 & fracar & 11.3 & $\mathrm{~s}$ \\
\hline 68.3 & \multicolumn{3}{|c|}{ marker \# 4} \\
\hline 69.6 & gleaqu & 16.7 & $\mathrm{n}$ \\
\hline 69.9 & fracar & 10.1 & $\mathrm{n}$ \\
\hline \multirow{2}{*}{70.0} & \multirow{2}{*}{ fracar } & 11.9 & \multirow{2}{*}{$\mathrm{n}$} \\
\hline & & 5.5 & \\
\hline 70.1 & betnig & 24.7 & $\mathrm{n}$ \\
\hline 70.1 & fracar & 14.3 & $\mathrm{n}$ \\
\hline \multirow{2}{*}{71.1} & \multirow{2}{*}{ plaaqu } & 16.0 & \multirow{2}{*}{$\mathrm{n}$} \\
\hline & & 4.3 & \\
\hline 71.5 & fracar & 12.1 & $\mathrm{~s}$ \\
\hline 72.5 & gleaqu & 20.2 & $\mathrm{~s}$ \\
\hline 73.5 & plaaqu & 13.8 & $\mathrm{n}$ \\
\hline \multirow{2}{*}{74.5} & \multirow{2}{*}{ fracar } & 13.4 & \multirow{2}{*}{$\mathrm{n}$} \\
\hline & & 7.1 & \\
\hline 75.9 & fracar & 14.6 & $\mathrm{n}$ \\
\hline \multirow{2}{*}{76.8} & \multirow{2}{*}{ gleaqu } & 25.6 & \multirow{2}{*}{$\mathrm{n}$} \\
\hline & & 20.0 & \\
\hline \multirow{2}{*}{77.9} & \multirow{2}{*}{ fracar } & 10.9 & \multirow{2}{*}{$\mathrm{s}$} \\
\hline & & 12.6 & \\
\hline 80.0 & taxdis & 17.9 & $\mathrm{n}$ \\
\hline 80.0 & taxdis & 17.8 & $\mathrm{~s}$ \\
\hline \multirow{5}{*}{80.5} & \multirow{5}{*}{ fracar } & 10.7 & \multirow{5}{*}{$\mathrm{s}$} \\
\hline & & 7.8 & \\
\hline & & 6.3 & \\
\hline & & 5.9 & \\
\hline & & 8.1 & \\
\hline 81.0 & plaaqu & 18.7 & $\mathrm{~s}$ \\
\hline \multirow{3}{*}{81.2} & & 10.6 & \\
\hline & fracar & 7.2 & $\mathrm{n}$ \\
\hline & & 6.9 & \\
\hline 82.8 & fracar & 14.4 & $\mathrm{~s}$ \\
\hline 83.8 & taxdis & 17.2 & $\mathrm{n}$ \\
\hline 84.0 & taxdis & 18.9 & $\mathrm{n}$ \\
\hline 846 & plogou & 22.8 & $e_{-1}$ \\
\hline 84.0 & práaqu & 10.8 & $\mathrm{~s}$ \\
\hline 85.6 & snag & 12.1 & $\mathrm{~s}$ \\
\hline 85.8 & plaaqu & 17.2 & $\mathrm{~s}$ \\
\hline
\end{tabular}

\begin{tabular}{|c|c|c|c|}
\hline $\begin{array}{l}\text { Distance from } \\
\text { beginning of } \\
\text { segment in } m\end{array}$ & $\begin{array}{c}\text { Tree } \\
\text { species }\end{array}$ & $\begin{array}{l}\text { Dbh, } \\
\text { in cm }\end{array}$ & Side \\
\hline 86.6 & salcar & 11.0 & $\mathrm{~s}$ \\
\hline 87.3 & salcar & 10.1 & $\mathrm{n}$ \\
\hline 87.9 & salcar & 12.6 & $\mathrm{~s}$ \\
\hline 88.6 & \multicolumn{3}{|c|}{ marker \# 5} \\
\hline 89.9 & betnig & 12.5 & $\mathrm{n}$ \\
\hline 89.9 & salcar & 15.7 & $\mathrm{~s}$ \\
\hline 91.1 & salnig & 12.6 & $\mathrm{n}$ \\
\hline 95.1 & quelyr & 24.1 & $\mathrm{n}$ \\
\hline 99.6 & quelau & 14.2 & $\mathrm{n}$ \\
\hline 100.8 & liqsty & 23.0 & $\mathrm{n}$ \\
\hline 101.1 & quelau & 25.6 & $\mathrm{n}$ \\
\hline 102.0 & liqsty & 18.6 & $\mathrm{n}$ \\
\hline 102.1 & liqsty & 20.0 & $\mathrm{~s}$ \\
\hline 102.3 & quelau & 17.5 & $\mathrm{n}$ \\
\hline 102.3 & taxdis & 18.8 & $\mathrm{~s}$ \\
\hline 102.8 & quelau & 12.1 & $\mathrm{n}$ \\
\hline 102.8 & taxdis & 16.9 & $\mathrm{~s}$ \\
\hline \multirow{2}{*}{103.1} & \multirow{2}{*}{ snag } & 19.4 & \multirow{2}{*}{$\mathrm{s}$} \\
\hline & & 17.5 & \\
\hline 104.3 & quelau & 20.7 & $\mathrm{~s}$ \\
\hline \multirow{2}{*}{105.4} & \multirow{2}{*}{ betnig } & 12.8 & \multirow{2}{*}{$\mathrm{s}$} \\
\hline & & 11.7 & \\
\hline 105.4 & taxdis & 13.1 & $\mathrm{n}$ \\
\hline 105.6 & betnig & 13.9 & $\mathrm{~s}$ \\
\hline 115.5 & \multicolumn{3}{|c|}{ marker \# 6} \\
\hline 115.9 & quelau & 35.2 & $\mathrm{~s}$ \\
\hline 120.0 & quelau & 25.4 & $\mathrm{~s}$ \\
\hline \multirow{2}{*}{120.5} & \multirow{2}{*}{ cyrrac } & 10.0 & \multirow{2}{*}{$\mathrm{n}$} \\
\hline & & 6.8 & \\
\hline \multirow{2}{*}{123.5} & \multirow{2}{*}{ nyssyl } & 27.5 & \multirow{2}{*}{$\mathrm{n}$} \\
\hline & & 25.0 & \\
\hline 128.3 & \multicolumn{3}{|c|}{ marker \# 7} \\
\hline \multicolumn{4}{|c|}{ East Segment } \\
\hline 0.0 & \multicolumn{3}{|c|}{ marker \# 8} \\
\hline \multirow{3}{*}{0.6} & \multirow{3}{*}{ cravir } & 10.6 & \multirow{3}{*}{$\mathrm{s}$} \\
\hline & & 10.1 & \\
\hline & & 6.0 & \\
\hline 2.2 & betnig & 14.6 & $\mathrm{~s}$ \\
\hline 2.7 & quelau & 57.4 & $\mathrm{n}$ \\
\hline 3.9 & betnig & 14.4 & $\mathrm{~s}$ \\
\hline 4.3 & quelau & 47.4 & $\mathrm{n}$ \\
\hline 5.8 & taxdis & 24.0 & $\mathrm{n}$ \\
\hline 10.6 & betnig & 20.0 & $\mathrm{~s}$ \\
\hline 10.6 & betnig & 16.2 & $\mathrm{~s}$ \\
\hline 12.2 & gleaqu & 22.1 & $\mathrm{~s}$ \\
\hline 12.9 & liqsty & 23.9 & $\mathrm{~s}$ \\
\hline 13.3 & taxdis & 34.5 & $\mathrm{~s}$ \\
\hline
\end{tabular}


A CF: The sampling width of this transect is 10 meters, 5 meters on either side of the transect line (Continued)

\begin{tabular}{|c|c|c|c|}
\hline $\begin{array}{l}\text { Distance from } \\
\text { beginning of } \\
\text { segment, in } \mathrm{m}\end{array}$ & $\begin{array}{c}\text { Tree } \\
\text { species }\end{array}$ & $\begin{array}{l}\text { Dbh, } \\
\text { in cm }\end{array}$ & Side \\
\hline 13.7 & liqsty & 10.0 & $\mathrm{n}$ \\
\hline 13.8 & taxdis & 29.0 & $\mathrm{~s}$ \\
\hline 14.8 & taxdis & 17.5 & $\mathrm{n}$ \\
\hline 15.2 & taxdis & 24.6 & $\mathrm{n}$ \\
\hline 16.1 & plaaqu & 36.0 & $\mathrm{~s}$ \\
\hline \multirow{3}{*}{16.5} & \multirow{3}{*}{ plaaqu } & 25.3 & \multirow{3}{*}{$\mathrm{n}$} \\
\hline & & 6.6 & \\
\hline & & 5.9 & \\
\hline 17.3 & taxdis & 22.4 & $\mathrm{n}$ \\
\hline 17.3 & taxdis & 23.9 & $\mathrm{n}$ \\
\hline 17.3 & taxdis & 48.0 & $\mathrm{n}$ \\
\hline \multirow{4}{*}{18.0} & \multirow{4}{*}{ plaaqu } & 22.0 & \multirow{4}{*}{$\mathrm{n}$} \\
\hline & & 22.1 & \\
\hline & & 9.0 & \\
\hline & & 20.0 & \\
\hline \multirow{2}{*}{19.9} & \multirow{2}{*}{ fracar } & 12.6 & \multirow{2}{*}{$\mathrm{s}$} \\
\hline & & 9.6 & \\
\hline 22.1 & \multicolumn{2}{|c|}{ marker \# 9} & \\
\hline 23.7 & acerub & 21.9 & $\mathrm{~s}$ \\
\hline 24.1 & snag & 20.0 & $\mathrm{n}$ \\
\hline 24.5 & acerub & 10.5 & $\mathrm{~s}$ \\
\hline 24.7 & gleaqu & 14.4 & $\mathrm{~s}$ \\
\hline 26.5 & betnig & 21.1 & $\mathrm{n}$ \\
\hline \multirow{3}{*}{28.5} & \multirow{3}{*}{ fracar } & 9.5 & \multirow{3}{*}{$\mathrm{s}$} \\
\hline & & 11.1 & \\
\hline & & 8.6 & \\
\hline \multirow{9}{*}{29.5} & \multirow{9}{*}{ fracar } & 11.6 & \multirow{9}{*}{$\mathrm{s}$} \\
\hline & & 9.3 & \\
\hline & & 4.1 & \\
\hline & & 6.5 & \\
\hline & & 6.4 & \\
\hline & & 9.6 & \\
\hline & & 8.0 & \\
\hline & & 4.7 & \\
\hline & & 6.5 & \\
\hline 33.7 & gleaqu & 15.5 & $\mathrm{n}$ \\
\hline 34.1 & betnig & 12.6 & $\mathrm{~s}$ \\
\hline 34.5 & cepocc & 12.5 & $\mathrm{~s}$ \\
\hline \multirow{2}{*}{37.4} & \multirow{2}{*}{ taxdis } & 30.9 & \multirow{2}{*}{$\mathrm{s}$} \\
\hline & & 15.3 & \\
\hline \multirow{5}{*}{38.1} & \multirow{5}{*}{ plaaqu } & 19.9 & \multirow{5}{*}{$\mathrm{s}$} \\
\hline & & 10.4 & \\
\hline & & 4.8 & \\
\hline & & 7.5 & \\
\hline & & 8.0 & \\
\hline
\end{tabular}

\begin{tabular}{|c|c|c|c|}
\hline $\begin{array}{l}\text { Distance from } \\
\text { beginning of } \\
\text { segment in } \mathrm{m}\end{array}$ & $\begin{array}{c}\text { Tree } \\
\text { species }\end{array}$ & $\begin{array}{l}\text { Dbh, } \\
\text { in cm }\end{array}$ & Side \\
\hline \multirow{4}{*}{39.1} & \multirow{4}{*}{ taxdis } & 29.7 & \multirow{4}{*}{$\mathrm{n}$} \\
\hline & & 30.0 & \\
\hline & & 10.5 & \\
\hline & & 39.0 & \\
\hline \multirow{4}{*}{39.9} & \multirow{4}{*}{ taxdis } & 31.5 & \multirow{4}{*}{$\mathrm{s}$} \\
\hline & & 24.3 & \\
\hline & & 17.3 & \\
\hline & & 28.5 & \\
\hline 40.8 & plaaqu & 34.0 & $\mathrm{~s}$ \\
\hline \multirow{3}{*}{42.1} & \multirow{3}{*}{ taxdis } & 37.1 & \multirow{3}{*}{$\mathrm{n}$} \\
\hline & & 29.0 & \\
\hline & & 12.8 & \\
\hline \multirow{3}{*}{47.1} & \multirow{3}{*}{ fracar } & 12.2 & \multirow{3}{*}{$\mathrm{s}$} \\
\hline & & 8.0 & \\
\hline & & 11.5 & \\
\hline \multirow{6}{*}{47.3} & \multirow{6}{*}{ taxdis } & 11.1 & \multirow{6}{*}{$\mathrm{n}$} \\
\hline & & 27.5 & \\
\hline & & 26.7 & \\
\hline & & 22.8 & \\
\hline & & 21.4 & \\
\hline & & 25.6 & \\
\hline 48.9 & betnig & 13.7 & $\mathrm{~s}$ \\
\hline \multirow{5}{*}{49.4} & \multirow{5}{*}{ fracar } & 11.2 & \multirow{5}{*}{$\mathrm{s}$} \\
\hline & & 8.5 & \\
\hline & & 12.3 & \\
\hline & & 6.4 & \\
\hline & & 5.8 & \\
\hline \multirow{5}{*}{53.3} & \multirow{5}{*}{ fracar } & 9.2 & \multirow{5}{*}{$\mathrm{n}$} \\
\hline & & 6.2 & \\
\hline & & 9.7 & \\
\hline & & 7.4 & \\
\hline & & 7.2 & \\
\hline 53.7 & taxdis & 19.7 & $\mathrm{n}$ \\
\hline 53.9 & \multicolumn{3}{|c|}{ marker \# 10} \\
\hline 54.2 & betnig & 11.9 & $\mathrm{n}$ \\
\hline \multirow{3}{*}{56.0} & \multirow{3}{*}{ taxdis } & 23.3 & \\
\hline & & 21.0 & $\mathrm{n}$ \\
\hline & & 27.7 & \\
\hline & & 12.5 & \\
\hline 566 & taxdis & 24.3 & $n$ \\
\hline 50.0 & taxais & 30.1 & $\mathrm{n}$ \\
\hline & & 27.9 & \\
\hline & & 19.4 & \\
\hline 500 & to dio & 17.2 & \\
\hline 58.0 & taxdis & 38.0 & S \\
\hline & & 17.0 & \\
\hline
\end{tabular}


A CF: The sampling width of this transect is 10 meters, 5 meters on either side of the transect line (Continued)

\begin{tabular}{|c|c|c|c|c|c|c|c|}
\hline $\begin{array}{l}\text { Distance from } \\
\text { beginning of } \\
\text { segment, in } \mathrm{m}\end{array}$ & $\begin{array}{c}\text { Tree } \\
\text { species }\end{array}$ & $\begin{array}{l}\text { Dbh, } \\
\text { in cm }\end{array}$ & Side & $\begin{array}{l}\text { Distance from } \\
\text { beginning of } \\
\text { segment in } \mathrm{m}\end{array}$ & $\begin{array}{c}\text { Tree } \\
\text { species }\end{array}$ & $\begin{array}{l}\text { Dbh, } \\
\text { in cm }\end{array}$ & Side \\
\hline 58.5 & taxdis & 40.8 & $\mathrm{~s}$ & \multirow{6}{*}{102.4} & \multirow{6}{*}{ fracar } & 10.4 & \multirow{6}{*}{$\mathrm{s}$} \\
\hline 59.1 & taxdis & 44.7 & $\mathrm{n}$ & & & 6.0 & \\
\hline 60.4 & taxdis & 23.0 & $\mathrm{n}$ & & & 10.8 & \\
\hline 61.5 & betnig & 10.7 & $\mathrm{n}$ & & & 7.4 & \\
\hline 63.2 & plaaqu & 42.3 & $\mathrm{~s}$ & & & 8.4 & \\
\hline 64.1 & betnig & 12.4 & $\mathrm{~s}$ & & & 5.7 & \\
\hline 65.0 & taxdis & 40.6 & $\mathrm{~s}$ & \multirow{4}{*}{103.7} & \multirow{4}{*}{ fracar } & 12.3 & \multirow{4}{*}{$\mathrm{n}$} \\
\hline \multirow{9}{*}{69.7} & \multirow{9}{*}{ fracar } & 10.6 & \multirow{9}{*}{$\mathrm{n}$} & & & 12.4 & \\
\hline & & 5.4 & & & & 12.5 & \\
\hline & & 8.9 & & & & 4.7 & \\
\hline & & 7.5 & & \multirow{4}{*}{105.0} & \multirow{4}{*}{ fracar } & 12.3 & \multirow{4}{*}{$\mathrm{n}$} \\
\hline & & 9.4 & & & & 7.3 & \\
\hline & & 6.9 & & & & 10.1 & \\
\hline & & 8.2 & & & & 8.9 & \\
\hline & & 10.0 & & 106.8 & & rker \# 12 & \\
\hline & & 9.9 & & 107.8 & taxdis & 25.5 & $\mathrm{~s}$ \\
\hline \multirow{3}{*}{71.2} & \multirow{3}{*}{ taxdis } & 27.8 & \multirow{3}{*}{$\mathrm{n}$} & 107.9 & taxdis & 27.3 & $\mathrm{n}$ \\
\hline & & 25.9 & & 107.9 & taxdis & 37.4 & $\mathrm{n}$ \\
\hline & & 23.8 & & 108.9 & taxdis & 32.6 & $\mathrm{n}$ \\
\hline 79.2 & taxdis & 45.2 & $\mathrm{n}$ & 109.9 & taxdis & 33.0 & $\mathrm{~s}$ \\
\hline 79.9 & \multirow{4}{*}{ taxdis } & arker \# & & 110.3 & gleaqu & 18.6 & $\mathrm{n}$ \\
\hline \multirow{3}{*}{79.8} & & 12.8 & & \multirow{6}{*}{110.8} & \multirow{6}{*}{ fracar } & 8.0 & \multirow{6}{*}{$\mathrm{n}$} \\
\hline & & 29.0 & $\mathrm{n}$ & & & 10.2 & \\
\hline & & 37.1 & & & & 11.5 & \\
\hline 84.1 & taxdis & 42.5 & $\mathrm{n}$ & & & 4.9 & \\
\hline & & 4.5 & & & & 9.0 & \\
\hline & & 8.6 & & & & 5.2 & \\
\hline & & 11.4 & & 113.8 & plaaqu & 14.4 & $\mathrm{n}$ \\
\hline & & 12.3 & & 114.2 & betnig & 20.0 & $\mathrm{n}$ \\
\hline & & 12.5 & & & & 12.9 & \\
\hline 88.3 & plaaqu & 8.7 & $\mathrm{n}$ & 114.4 & plaaqu & 6.0 & $\mathrm{~s}$ \\
\hline & & 8.3 & & 116.2 & caraqu & 14.5 & $\mathrm{~s}$ \\
\hline & & 21.6 & & 117.3 & plaaqu & 14.8 & $\mathrm{~s}$ \\
\hline & & 10.3 & & 117.5 & gleaqu & 11.7 & $\mathrm{n}$ \\
\hline & & 16.9 & & 117.7 & plaaqu & 17.6 & $\mathrm{n}$ \\
\hline 88.4 & taxdis & 35.0 & $\mathrm{~s}$ & 1170 & hatni & 15.5 & \\
\hline 88.9 & taxdis & 30.4 & $\mathrm{n}$ & 117.9 & betnig & 12.1 & s \\
\hline 92.7 & taxdis & 32.6 & $\mathrm{n}$ & & & 20.5 & \\
\hline & & 18.8 & & 118.0 & plaaqu & 7.0 & $\mathrm{n}$ \\
\hline 95.1 & plaaqu & 8.8 & $\mathrm{~s}$ & & & 12.5 & \\
\hline 95.8 & betnig & 10.6 & $\mathrm{~s}$ & 1100 & froco & 8.0 & n \\
\hline 97.7 & taxdis & 44.5 & $\mathrm{n}$ & 119.8 & fracar & 8.6 & $\mathrm{n}$ \\
\hline 99.1 & taxdis & 35.6 & $\mathrm{n}$ & & & 7.4 & \\
\hline & & 19.9 & & & & 12.4 & \\
\hline & & 26.4 & & 120.4 & fracar & 9.6 & $\mathrm{n}$ \\
\hline & & 26.0 & & & & 4.0 & \\
\hline 100.4 & taxdis & 27.3 & $\mathrm{~s}$ & 121.4 & plaaqu & 10.1 & $\mathrm{~s}$ \\
\hline & & 29.1 & & 122.7 & taxdis & 45.2 & $\mathrm{n}$ \\
\hline & & 24.9 & & 123.1 & gleaqu & 27.4 & $\mathrm{~s}$ \\
\hline
\end{tabular}


A CF: The sampling width of this transect is 10 meters, 5 meters on either side of the transect line (Continued)

\begin{tabular}{|c|c|c|c|}
\hline $\begin{array}{l}\text { Distance from } \\
\text { beginning of } \\
\text { segment, in } \mathrm{m}\end{array}$ & $\begin{array}{c}\text { Tree } \\
\text { species }\end{array}$ & $\begin{array}{l}\text { Dbh, } \\
\text { in } \mathrm{cm}\end{array}$ & Side \\
\hline 124.5 & acerub & 14.3 & $\mathrm{n}$ \\
\hline 128.5 & quelau & 10.5 & $\mathrm{n}$ \\
\hline 129.4 & \multicolumn{3}{|c|}{ marker \# 13} \\
\hline 130.1 & liqsty & 45.5 & $\mathrm{n}$ \\
\hline 130.6 & carcar & 15.5 & $\mathrm{n}$ \\
\hline 131.4 & betnig & 35.9 & $\mathrm{n}$ \\
\hline 131.4 & liqsty & 23.3 & $\mathrm{~s}$ \\
\hline 133.0 & snag & 10.9 & $\mathrm{~s}$ \\
\hline 133.2 & ulmame & 18.0 & $\mathrm{n}$ \\
\hline 135.9 & ulmame & 19.5 & $\mathrm{n}$ \\
\hline \multirow{2}{*}{136.1} & \multirow{2}{*}{ carcar } & 12.3 & \multirow{2}{*}{$\mathrm{s}$} \\
\hline & & 6.4 & \\
\hline 141.7 & liqsty & 23.5 & $\mathrm{~s}$ \\
\hline 144.3 & liqsty & 30.0 & $\mathrm{n}$ \\
\hline 146.4 & taxdis & 36.4 & $\mathrm{~s}$ \\
\hline \multirow{2}{*}{147.9} & \multirow{2}{*}{ carcar } & 21.1 & \multirow{2}{*}{$\mathrm{n}$} \\
\hline & & 6.8 & \\
\hline 148.8 & carcar & 10.3 & $\mathrm{~s}$ \\
\hline 149.9 & carcar & 15.8 & $\mathrm{n}$ \\
\hline 154.1 & carcar & 13.8 & $\mathrm{n}$ \\
\hline 156.6 & carcar & 13.9 & $\mathrm{~s}$ \\
\hline 157.3 & quevir & 43.8 & $\mathrm{~s}$ \\
\hline 158.7 & \multicolumn{3}{|c|}{ marker \# 14} \\
\hline 158.8 & liqsty & 10.4 & $\mathrm{n}$ \\
\hline 159.1 & quevir & 65.5 & $\mathrm{n}$ \\
\hline 161.7 & snag & 57.8 & $\mathrm{n}$ \\
\hline 162.6 & liqsty & 11.9 & $\mathrm{~s}$ \\
\hline 167.4 & liqsty & 11.8 & $\mathrm{~s}$ \\
\hline 167.5 & cargla & 27.0 & $\mathrm{n}$ \\
\hline 168.3 & liqsty & 15.6 & $\mathrm{~s}$ \\
\hline 169.3 & quenig & 31.7 & $\mathrm{n}$ \\
\hline \multirow{2}{*}{172.0} & \multirow{2}{*}{ carcar } & 20.3 & \multirow{2}{*}{$\mathrm{n}$} \\
\hline & & 4.0 & \\
\hline 176.3 & liqsty & 13.8 & $\mathrm{n}$ \\
\hline 177.0 & liqsty & 13.6 & $\mathrm{~s}$ \\
\hline 179.0 & carcar & 11.4 & $\mathrm{n}$ \\
\hline 180.7 & carcar & 15.9 & $\mathrm{n}$ \\
\hline 181.6 & carcar & 16.0 & $\mathrm{n}$ \\
\hline 182.8 & nysbif & 11.9 & $\mathrm{~s}$ \\
\hline 183.0 & carcar & 12.0 & $\mathrm{n}$ \\
\hline 184.0 & carcar & 13.1 & $\mathrm{~s}$ \\
\hline 184.4 & carcar & 15.0 & $\mathrm{~s}$ \\
\hline 186.5 & carcar & 14.3 & $\mathrm{~s}$ \\
\hline 186.6 & carcar & 12.5 & $\mathrm{~s}$ \\
\hline 187.8 & & rker \# & \\
\hline
\end{tabular}

\begin{tabular}{|c|c|c|c|}
\hline $\begin{array}{l}\text { Distance from } \\
\text { beginning of } \\
\text { segment in } \mathrm{m}\end{array}$ & $\begin{array}{c}\text { Tree } \\
\text { species }\end{array}$ & $\begin{array}{l}\text { Dbh, } \\
\text { in cm }\end{array}$ & Side \\
\hline 189.0 & carcar & 10.2 & $\mathrm{n}$ \\
\hline 194.9 & liqsty & 28.4 & $\mathrm{n}$ \\
\hline 198.7 & carcar & 15.7 & $\mathrm{n}$ \\
\hline 201.4 & liqsty & 11.8 & $\mathrm{n}$ \\
\hline 201.6 & quenig & 43.8 & $\mathrm{n}$ \\
\hline 202.1 & liqsty & 12.9 & $\mathrm{n}$ \\
\hline 205.5 & liqsty & 17.4 & $\mathrm{n}$ \\
\hline 206.2 & snag & 24.0 & $\mathrm{~s}$ \\
\hline \multirow{2}{*}{208.3} & \multirow{2}{*}{ carcar } & 14.4 & \multirow{2}{*}{$\mathrm{s}$} \\
\hline & & 14.5 & \\
\hline 210.4 & snag & 20.0 & $\mathrm{n}$ \\
\hline 211.1 & carcar & 13.3 & $\mathrm{n}$ \\
\hline 212.7 & liqsty & 35.1 & $\mathrm{n}$ \\
\hline 213.8 & carcar & 10.9 & $\mathrm{~s}$ \\
\hline 214.4 & ulmala & 18.7 & $\mathrm{~s}$ \\
\hline 215.5 & acerub & 20.6 & $\mathrm{n}$ \\
\hline 216.3 & \multicolumn{3}{|c|}{ marker \# 16} \\
\hline 218.3 & quenig & 36.0 & $\mathrm{~s}$ \\
\hline 220.3 & ileopa & 11.4 & $\mathrm{n}$ \\
\hline 221.4 & carcar & 12.5 & $\mathrm{~s}$ \\
\hline 232.7 & nysbif & 16.8 & $\mathrm{~s}$ \\
\hline 233.8 & nysbif & 32.5 & $\mathrm{~s}$ \\
\hline 234.9 & carcar & 20.5 & $\mathrm{n}$ \\
\hline 235.2 & \multicolumn{3}{|c|}{ marker \# 17} \\
\hline 241.0 & liqsty & 29.0 & $\mathrm{~s}$ \\
\hline 243.5 & taxdis & 17.7 & $\mathrm{n}$ \\
\hline 243.7 & quelau & 28.0 & $\mathrm{n}$ \\
\hline 244.8 & liqsty & 36.1 & $\mathrm{~s}$ \\
\hline 246.6 & taxdis & 17.8 & $\mathrm{~s}$ \\
\hline 251.7 & taxdis & 41.6 & $\mathrm{n}$ \\
\hline 252.7 & liqsty & 10.8 & $\mathrm{n}$ \\
\hline 252.8 & cravir & 18.6 & $\mathrm{n}$ \\
\hline 253.5 & taxdis & 12.5 & $\mathrm{~s}$ \\
\hline \multirow{6}{*}{255.9} & \multirow{6}{*}{ fracar } & 7.0 & \multirow{6}{*}{$\mathrm{s}$} \\
\hline & & 14.3 & \\
\hline & & 5.3 & \\
\hline & & 4.4 & \\
\hline & & 7.0 & \\
\hline & & 7.8 & \\
\hline 256.7 & \multicolumn{3}{|c|}{ marker \# 18} \\
\hline \multirow{2}{*}{257.6} & \multirow{2}{*}{ plaaqu } & 52.4 & \multirow[b]{2}{*}{$\mathrm{s}$} \\
\hline & & 30.7 & \\
\hline 258.0 & taxdis & 33.9 & $\mathrm{~s}$ \\
\hline 260.2 & quelyr & 51.5 & $\mathrm{n}$ \\
\hline
\end{tabular}


A CF: The sampling width of this transect is 10 meters, 5 meters on either side of the transect line (Continued)

\begin{tabular}{|c|c|c|c|}
\hline $\begin{array}{l}\text { Distance from } \\
\text { beginning of } \\
\text { segment, in } \mathrm{m}\end{array}$ & Tree species & $\begin{array}{l}\text { Dbh, } \\
\text { in } \mathrm{cm}\end{array}$ & Side \\
\hline \multirow{3}{*}{262.9} & \multirow{3}{*}{ fracar } & 4.5 & \multirow{3}{*}{$\mathrm{n}$} \\
\hline & & 8.4 & \\
\hline & & 11.6 & \\
\hline 266.6 & plaaqu & 35.0 & $\mathrm{~s}$ \\
\hline \multirow{3}{*}{268.0} & \multirow{3}{*}{ plaaqu } & 29.7 & \multirow{3}{*}{$\mathrm{n}$} \\
\hline & & 6.0 & \\
\hline & & 13.2 & \\
\hline 270.3 & plaaqu & 24.0 & $\mathrm{n}$ \\
\hline 277.2 & ulmame & 19.0 & $\mathrm{n}$ \\
\hline 280.1 & ulmame & 15.9 & $\mathrm{~s}$ \\
\hline 281.2 & ulmame & 17.7 & $\mathrm{~s}$ \\
\hline 284.7 & acerub & 10.3 & $\mathrm{n}$ \\
\hline 286.1 & \multicolumn{3}{|c|}{ marker \# 19} \\
\hline 288.1 & quelau & 17.6 & $\mathrm{~s}$ \\
\hline 289.5 & caraqu & 11.9 & $\mathrm{~s}$ \\
\hline 290.6 & acerub & 13.5 & $\mathrm{n}$ \\
\hline 293.5 & betnig & 15.5 & $\mathrm{~s}$ \\
\hline 294.1 & carcar & 15.4 & $\mathrm{n}$ \\
\hline 296.3 & liqsty & 11.8 & $\mathrm{n}$ \\
\hline 298.5 & liqsty & 14.4 & $\mathrm{n}$ \\
\hline 300.7 & liqsty & 11.8 & $\mathrm{n}$ \\
\hline 300.8 & \multicolumn{3}{|c|}{ marker \# 20} \\
\hline 301.5 & carcar & 13.6 & $\mathrm{~s}$ \\
\hline 302.5 & carcar & 22.7 & $\mathrm{n}$ \\
\hline 302.6 & carcar & 25.2 & $\mathrm{~s}$ \\
\hline 304.7 & snag & 81.0 & $\mathrm{~s}$ \\
\hline 308.1 & betnig & 17.1 & $\mathrm{n}$ \\
\hline 308.1 & betnig & 17.5 & $\mathrm{n}$ \\
\hline 308.5 & salcar & 11.8 & $\mathrm{~s}$ \\
\hline 310.7 & \multicolumn{3}{|c|}{ marker \# 21} \\
\hline 311.1 & betnig & 10.0 & $\mathrm{n}$ \\
\hline 312.7 & taxdis & 45.0 & $\mathrm{n}$ \\
\hline 312.7 & taxdis & 62.0 & $\mathrm{~s}$ \\
\hline
\end{tabular}


B LL: The sampling width of this transect is 5 meters on the west side of the transect line.

\begin{tabular}{|c|c|c|c|c|c|c|c|}
\hline $\begin{array}{l}\text { Distance from } \\
\text { beginning of } \\
\text { transect, in } \mathrm{m}\end{array}$ & $\begin{array}{c}\text { Tree } \\
\text { species }\end{array}$ & $\begin{array}{l}\text { Dbh, } \\
\text { in cm }\end{array}$ & Side & $\begin{array}{l}\text { Distance from } \\
\text { beginning of } \\
\text { transect, in } \mathrm{m}\end{array}$ & $\begin{array}{c}\text { Tree } \\
\text { species }\end{array}$ & $\begin{array}{l}\text { Dbh, } \\
\text { in } \mathrm{cm}\end{array}$ & Side \\
\hline-4.6 & betnig & 14.0 & $\mathrm{~W}$ & 110.4 & taxdis & 35.7 & $\mathrm{~W}$ \\
\hline \multirow{2}{*}{-4.5} & \multirow{2}{*}{ betnig } & 13.0 & \multirow{2}{*}{$\mathrm{w}$} & 110.6 & \multicolumn{3}{|c|}{ marker \# 26} \\
\hline & & 21.7 & & 113.6 & \multicolumn{3}{|c|}{ marker \# 27} \\
\hline 0.0 & \multicolumn{3}{|c|}{ marker \# 22} & 114.5 & plaaqu & 41.2 & $\mathrm{w}$ \\
\hline \multirow{7}{*}{20.3} & \multirow{7}{*}{ foracu } & 11.3 & \multirow{7}{*}{$\mathrm{w}$} & \multirow{2}{*}{121.9} & \multirow{2}{*}{ foracu } & 14.5 & \multirow{2}{*}{ w } \\
\hline & & 5.1 & & & & 8.2 & \\
\hline & & 6.4 & & 122.8 & foracu & 11.8 & $\mathrm{w}$ \\
\hline & & 8.7 & & \multirow{3}{*}{124.9} & \multirow{3}{*}{ foracu } & 12.2 & \multirow{3}{*}{$\mathrm{w}$} \\
\hline & & 4.2 & & & & 7.1 & \\
\hline & & 8.2 & & & & 10.9 & \\
\hline & & 7.7 & & 127.4 & foracu & 10.2 & $\mathrm{~W}$ \\
\hline 29.8 & plaaqu & 24.6 & $\mathrm{w}$ & 134.0 & foracu & 15.7 & $\mathrm{w}$ \\
\hline 31.8 & & $r$ \# 23 & & 140.3 & & \# 28 & \\
\hline \multirow{3}{*}{32.0} & \multirow{3}{*}{ foracu } & 10.4 & \multirow{3}{*}{$\mathrm{w}$} & 141.7 & caraqu & 59.4 & $\mathrm{~W}$ \\
\hline & & 4.3 & & 142.1 & foracu & 14.5 & $\mathrm{w}$ \\
\hline & & 5.9 & & 146.3 & cellae & 10.1 & $\mathrm{w}$ \\
\hline 39.1 & plaaqu & 29.6 & $\mathrm{~W}$ & 152.6 & foracu & 10.5 & $\mathrm{w}$ \\
\hline \multirow{3}{*}{39.6} & \multirow{3}{*}{ fracar } & 36.8 & \multirow{3}{*}{$\mathrm{w}$} & 161.7 & plaaqu & 57.3 & $\mathrm{w}$ \\
\hline & & 4.1 & & 162.0 & plaaqu & 37.1 & W \\
\hline & & 4.4 & & 162.9 & \multicolumn{3}{|c|}{ marker \# 29} \\
\hline 49.2 & plaaqu & 49.9 & w & & & 24.5 & \\
\hline 49.8 & gleaqu & 49.7 & W & 170.4 & fracar & 19.8 & W \\
\hline 50.4 & gleaqu & 58.9 & W & & & 4.2 & \\
\hline 52.3 & plaaqu & 51.1 & w & 1821 & ploogu & 14.8 & \\
\hline 53.0 & plaaqu & 32.7 & W & 105.1 & práqu & 6.1 & $\mathrm{w}$ \\
\hline 61.7 & taxdis & 45.7 & $\mathrm{~W}$ & & & 24.5 & \\
\hline 650 & jleder & 11.2 & $\mathrm{w}$ & 184.1 & plaaqu & 4.4 & W \\
\hline 05.9 & Hedec & 5.4 & W & & & 5.2 & \\
\hline 69.2 & & er \# 24 & & 184.2 & & \# 30 & \\
\hline & & 10.9 & & & & 19.5 & \\
\hline 84.1 & foracu & 7.4 & W & 1870 & froco & 5.8 & W \\
\hline & & 5.9 & & $18 \%$ & Iracar & 4.2 & W \\
\hline & & 16.7 & & & & 5.0 & \\
\hline & & 10.2 & & 190.4 & plaaqu & 19.9 & $\mathrm{~W}$ \\
\hline 888 & form & 5.9 & W & 1005 & plogou & 34.4 & W \\
\hline 00.0 & Ioracu & 10.0 & $\mathrm{~W}$ & 190.5 & pradqu & 20.5 & $\mathrm{~W}$ \\
\hline & & 4.5 & & 1071 & foron & 11.8 & is \\
\hline & & 4.7 & & 191.1 & Iracar & 4.9 & W \\
\hline & & 10.8 & & 200.5 & taxdis & 63.0 & $\mathrm{~W}$ \\
\hline 98.0 & cravir & 4.8 & w & & & 13.6 & \\
\hline & & 8.2 & & 204.2 & fracar & 9.8 & w \\
\hline 98.8 & plaaqu & 38.7 & $\mathrm{w}$ & & & 6.0 & \\
\hline 102.9 & betnig & 30.8 & $\mathrm{w}$ & & & 16.4 & \\
\hline 103.5 & gleaqu & 27.9 & $\mathrm{w}$ & 2047 & fracar & 8.0 & $w$ \\
\hline 104.5 & & er \# 25 & & 204.1 & 11 acal & 13.7 & $\mathrm{w}$ \\
\hline & & & & & & 4.2 & \\
\hline
\end{tabular}


B LL: The sampling width of this transect is 5 meters on the west side of the transect line.

\begin{tabular}{|c|c|c|c|c|c|c|c|}
\hline $\begin{array}{l}\text { Distance from } \\
\text { beginning of } \\
\text { transect, in } m\end{array}$ & $\begin{array}{c}\text { Tree } \\
\text { species }\end{array}$ & $\begin{array}{l}\text { Dbh, } \\
\text { in cm }\end{array}$ & Side & $\begin{array}{l}\text { Distance from } \\
\text { beginning of } \\
\text { transect, in } \mathrm{m}\end{array}$ & $\begin{array}{c}\text { Tree } \\
\text { species }\end{array}$ & $\begin{array}{l}\text { Dbh, } \\
\text { in cm }\end{array}$ & Side \\
\hline \multirow{4}{*}{206.3} & \multirow{4}{*}{ fracar } & 20.0 & \multirow{4}{*}{$\mathrm{w}$} & 294.4 & taxdis & 49.1 & $\mathrm{w}$ \\
\hline & & 16.0 & & 295.0 & fracar & 15.9 & $\mathrm{w}$ \\
\hline & & 14.9 & & 295.0 & fracar & 20.9 & $\mathrm{w}$ \\
\hline & & 4.8 & & 320.6 & taxdis & 71.4 & $\mathrm{w}$ \\
\hline 210.1 & taxdis & 29.2 & $\mathrm{w}$ & 324.2 & \multicolumn{3}{|c|}{ marker \# 34} \\
\hline \multirow{2}{*}{217.4} & \multirow{2}{*}{ fracar } & 18.7 & \multirow{2}{*}{$\mathrm{w}$} & \multirow{2}{*}{324.9} & \multirow{2}{*}{ plaaqu } & 12.7 & \multirow{2}{*}{$\mathrm{w}$} \\
\hline & & 6.0 & & & & 33.6 & \\
\hline 217.5 & fracar & 10.2 & $\mathrm{w}$ & \multirow{3}{*}{337.7} & \multirow{3}{*}{ fracar } & 15.8 & \multirow{3}{*}{$\mathrm{w}$} \\
\hline \multirow{3}{*}{220.2} & \multirow{3}{*}{ fracar } & 23.9 & & & & 15.8 & \\
\hline & & 13.9 & $\mathrm{w}$ & & & 17.4 & \\
\hline & & 14.5 & & 338.2 & taxdis & 44.7 & $\mathrm{w}$ \\
\hline \multirow{2}{*}{220.8} & \multirow{2}{*}{ fracar } & 13.3 & \multirow{2}{*}{$\mathrm{w}$} & 339.8 & taxdis & 63.4 & $\mathrm{w}$ \\
\hline & & 13.0 & & 341.9 & fracar & 26.6 & $\mathrm{w}$ \\
\hline \multirow{3}{*}{221.1} & \multirow{3}{*}{ fracar } & 16.0 & \multirow{3}{*}{$\mathrm{w}$} & 342.0 & fracar & 15.8 & $\mathrm{w}$ \\
\hline & & 12.6 & & 343.3 & taxdis & 56.6 & $\mathrm{w}$ \\
\hline & & 12.0 & & 349.2 & \multicolumn{3}{|c|}{ marker \# 35} \\
\hline 232.1 & taxdis & 58.4 & $\mathrm{w}$ & 350.7 & taxdis & 35.2 & W \\
\hline 2326 & fracar & 14.8 & & 354.2 & snag & 11.7 & $\mathrm{w}$ \\
\hline 232.0 & Iracar & 12.5 & W & & & 16.9 & \\
\hline 232.8 & fracar & 20.0 & W & & & 4.1 & \\
\hline 232.9 & fracar & 19.1 & W & & & 4.7 & \\
\hline 233.3 & fracar & 11.0 & W & 365.0 & fracar & 11.0 & W \\
\hline & & 23.3 & & & & 19.3 & \\
\hline & & 13.4 & & & & 20.7 & \\
\hline 239.0 & fracar & 13.6 & W & & & 19.8 & \\
\hline & & 20.5 & & & & 8.6 & \\
\hline & & 16.5 & & 365.5 & fracar & 14.3 & w \\
\hline 2402 & frocor & 16.8 & w & & & 11.8 & \\
\hline 240.2 & 11 acal & 5.8 & $\mathrm{~W}$ & 367.0 & taxdis & 57.2 & $\mathrm{w}$ \\
\hline 246.2 & & er \# 31 & & 372.2 & fracar & 23.0 & $\mathrm{w}$ \\
\hline 2570 & foroci & 10.0 & $\mathrm{~W}$ & 372.2 & fracar & 22.0 & $\mathrm{w}$ \\
\hline 251.2 & Ioracu & 8.1 & W & 373.9 & taxdis & 42.6 & w \\
\hline & & 4.8 & & 375.0 & taxdis & 52.8 & w \\
\hline 2633 & fracar & 4.3 & w & 375.6 & taxdis & 55.0 & w \\
\hline & Iracar & 18.2 & W & 2777 & fropo & 12.3 & 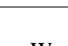 \\
\hline & & 18.5 & & 511.1 & $111 \mathrm{dcal}$ & 5.0 & W \\
\hline 270.7 & & er \# 32 & & 395.5 & plaaqu & 25.0 & w \\
\hline 271.8 & quelyr & 39.0 & $\mathrm{w}$ & 3070 & frocon & 23.4 & t \\
\hline 277.9 & taxdis & 20.8 & $\mathrm{w}$ & 391.2 & Iracar & 12.9 & $\mathrm{~W}$ \\
\hline 282.7 & & er \# 33 & & 398.0 & plaaqu & 32.5 & $\mathrm{w}$ \\
\hline 283.7 & cepocc & 17.6 & $\mathrm{w}$ & 400.3 & & \# 36 & \\
\hline 2854 & plag & 18.7 & $w$ & & & 17.0 & \\
\hline 205.4 & práqu & 8.7 & $\mathrm{~W}$ & 4 & nlongu & 11.7 & $\mathrm{w}$ \\
\hline 289.6 & taxdis & 42.9 & W & 402.4 & plaaqu & 14.9 & W \\
\hline 292.7 & taxdis & 18.2 & W & & & 10.6 & \\
\hline
\end{tabular}


B LL: The sampling width of this transect is 5 meters on the west side of the transect line.

\begin{tabular}{|c|c|c|c|c|c|c|c|}
\hline $\begin{array}{l}\text { Distance from } \\
\text { beginning of } \\
\text { transect, in } \mathrm{m}\end{array}$ & $\begin{array}{c}\text { Tree } \\
\text { species }\end{array}$ & $\begin{array}{l}\text { Dbh, } \\
\text { in cm }\end{array}$ & Side & $\begin{array}{l}\text { Distance from } \\
\text { beginning of } \\
\text { transect, in } \mathrm{m}\end{array}$ & $\begin{array}{c}\text { Tree } \\
\text { species }\end{array}$ & $\begin{array}{l}\text { Dbh, } \\
\text { in cm }\end{array}$ & Side \\
\hline 417.0 & \multicolumn{3}{|c|}{ marker \# 37} & 621.8 & plaaqu & 27.5 & $\mathrm{~W}$ \\
\hline 427.6 & liqsty & 49.7 & w & 629.8 & betnig & 51.0 & $\mathrm{w}$ \\
\hline 429.0 & cravir & 12.8 & w & \multirow{3}{*}{632.1} & \multirow{3}{*}{ iledec } & 6.2 & \multirow{3}{*}{ w } \\
\hline 437.7 & & H 38 & & & & 5.2 & \\
\hline 443.3 & betnig & 37.6 & $\mathrm{w}$ & & & 11.7 & \\
\hline 457.8 & taxdis & 13.8 & $\mathrm{w}$ & 639.6 & liqsty & 22.0 & $\mathrm{w}$ \\
\hline 458.7 & taxdis & 12.2 & $\mathrm{w}$ & 644.3 & ulmame & 40.1 & $\mathrm{w}$ \\
\hline 461.7 & diovir & 10.3 & $\mathrm{w}$ & 649.8 & caraqu & 36.8 & $\mathrm{w}$ \\
\hline 463.8 & snag & 65.0 & $\mathrm{~W}$ & 657.0 & betnig & 38.4 & $\mathrm{~W}$ \\
\hline 465.8 & cravir & 13.8 & w & 662.1 & betnig & 28.2 & $\mathrm{w}$ \\
\hline 467.7 & \multicolumn{3}{|c|}{ marker \# 39} & 667.1 & \multicolumn{3}{|c|}{ marker \# 44} \\
\hline 468.2 & liqsty & 11.4 & $\mathrm{w}$ & 674.8 & taxdis & 15.1 & $\mathrm{w}$ \\
\hline 472.4 & betnig & 13.2 & w & 683.1 & carcar & 16.2 & $\mathrm{w}$ \\
\hline 486.3 & \multicolumn{3}{|c|}{ marker \# 40} & 687.9 & carcar & 11.5 & $\mathrm{w}$ \\
\hline 491.1 & quelau & 68.0 & $\mathrm{w}$ & \multirow{2}{*}{693.7} & \multirow{2}{*}{ carcar } & 12.2 & \multirow{2}{*}{$\mathrm{w}$} \\
\hline 492.3 & acerub & 34.0 & w & & & 5.0 & \\
\hline 496.4 & liqsty & 20.3 & w & 698.4 & carcar & 10.9 & $\mathrm{~W}$ \\
\hline 496.5 & liqsty & 30.5 & w & 699.0 & & \# 45 & \\
\hline 498.9 & betnig & 10.9 & W & \multirow{5}{*}{705.4} & \multirow{5}{*}{ carcar } & 7.0 & \multirow{5}{*}{$\mathrm{W}$} \\
\hline \multirow{4}{*}{500.0} & \multirow{4}{*}{ fracar } & 4.6 & \multirow{4}{*}{ w } & & & 6.0 & \\
\hline & & 19.7 & & & & 6.5 & \\
\hline & & 5.5 & & & & 4.0 & \\
\hline & & 11.6 & & & & 13.0 & \\
\hline 518.0 & cravir & 11.0 & $\mathrm{w}$ & \multirow{2}{*}{712.3} & \multirow{2}{*}{ carcar } & 14.4 & \multirow{2}{*}{$\mathrm{w}$} \\
\hline 518.8 & quelau & 56.0 & $\mathrm{w}$ & & & 5.7 & \\
\hline 519.7 & cravir & 12.3 & $\mathrm{w}$ & 719.3 & \multicolumn{3}{|c|}{ marker \# 46} \\
\hline 522.6 & quelau & 52.2 & w & 726.7 & carcar & 11.0 & $\mathrm{w}$ \\
\hline 548.2 & \multicolumn{3}{|c|}{ marker \# 41} & 729.8 & quevir & 101.9 & $\mathrm{w}$ \\
\hline 548.5 & liqsty & 33.6 & $\mathrm{w}$ & 737.3 & carcar & 13.4 & $\mathrm{~W}$ \\
\hline 557.4 & carcar & 20.9 & $\mathrm{w}$ & 741.7 & carcar & 10.2 & $\mathrm{w}$ \\
\hline 561.9 & betnig & 14.4 & $\mathrm{w}$ & 750.6 & \multicolumn{3}{|c|}{ marker \# 47} \\
\hline 564.1 & betnig & 21.5 & $\mathrm{w}$ & 751.0 & carcar & 10.9 & w \\
\hline 569.0 & & r \# 42 & & 753.5 & liqsty & 16.9 & $\mathrm{w}$ \\
\hline 569.8 & taxdis & 31.7 & w & 756.8 & carcar & 12.7 & $\mathrm{w}$ \\
\hline 5700 & fropon & 14.2 & (1) & 762.9 & nyssyl & 38.4 & $\mathrm{w}$ \\
\hline $5 / 0.9$ & Iracar & 4.2 & W & 766.7 & snag & 65.0 & $\mathrm{w}$ \\
\hline 581.8 & taxdis & 13.9 & $\mathrm{w}$ & 768.0 & & \# 48 & \\
\hline 590.5 & betnig & 51.4 & $\mathrm{w}$ & 768.1 & carcar & 15.3 & $\mathrm{w}$ \\
\hline 592.4 & taxdis & 16.0 & w & 769.6 & nyssyl & 13.7 & w \\
\hline 598.3 & betnig & 41.7 & w & 778.8 & carcar & 23.9 & $\mathrm{w}$ \\
\hline 598.9 & ulmcra & 15.2 & w & 782.4 & nyssyl & 30.8 & $\mathrm{w}$ \\
\hline 599.1 & taxdis & 26.1 & w & 786.5 & nyssyl & 26.5 & w \\
\hline 612.6 & & $r \# 43$ & & 786.7 & carcar & 17.2 & w \\
\hline 614.8 & acerub & 32.5 & w & 786.7 & nyssyl & 25.5 & w \\
\hline 6107 & lo & 37.0 & 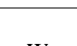 & 787.4 & carcar & 18.3 & w \\
\hline 618.7 & gleaqu & 8.2 & W & 787.9 & nyssyl & 30.0 & w \\
\hline
\end{tabular}


B LL: The sampling width of this transect is 5 meters on the west side of the transect line (Continued)

\begin{tabular}{|c|c|c|c|}
\hline $\begin{array}{l}\text { Distance from } \\
\text { beginning of } \\
\text { transect, in } \mathrm{m}\end{array}$ & $\begin{array}{c}\text { Tree } \\
\text { species }\end{array}$ & $\begin{array}{l}\text { Dbh, } \\
\text { in cm }\end{array}$ & Side \\
\hline 789.6 & carcar & 16.4 & $\mathrm{~W}$ \\
\hline 792.5 & carcar & 12.0 & $\mathrm{~W}$ \\
\hline 796.5 & carcar & 10.5 & $\mathrm{~W}$ \\
\hline 802.1 & carcar & 27.4 & W \\
\hline 809.9 & betnig & 39.9 & $\mathrm{~W}$ \\
\hline 811.2 & carcar & 10.8 & W \\
\hline 812.4 & liqsty & 16.1 & W \\
\hline 814.3 & liqsty & 39.3 & $\mathrm{w}$ \\
\hline 816.9 & liqsty & 13.4 & w \\
\hline 822.3 & liqsty & 19.6 & w \\
\hline 822.4 & \multicolumn{3}{|c|}{ marker \# 49} \\
\hline 826.6 & betnig & 22.8 & w \\
\hline 829.5 & carcar & 14.3 & w \\
\hline 833.6 & carcar & 11.5 & w \\
\hline 837.2 & carcar & 11.2 & W \\
\hline 837.5 & carcar & 12.2 & W \\
\hline 837.6 & betnig & 12.3 & $\mathrm{w}$ \\
\hline \multirow{2}{*}{845.2} & \multirow{2}{*}{ carcar } & 24.6 & \multirow{2}{*}{$\mathrm{w}$} \\
\hline & & 7.9 & \\
\hline 855.0 & carcar & 21.4 & W \\
\hline 857.4 & carcar & 21.1 & $\mathrm{w}$ \\
\hline 862.7 & carcar & 25.0 & $\mathrm{w}$ \\
\hline 863.3 & carcar & 15.5 & $\mathrm{w}$ \\
\hline 868.2 & \multicolumn{3}{|c|}{ marker \# 50} \\
\hline 872.4 & carcar & 20.8 & $\mathrm{w}$ \\
\hline 875.1 & quevir & 64.0 & W \\
\hline 880.8 & nyssyl & 21.9 & $\mathrm{~W}$ \\
\hline 881.2 & carcar & 12.0 & $\mathrm{~W}$ \\
\hline 884.1 & nyssyl & 25.8 & $\mathrm{~W}$ \\
\hline 884.4 & diovir & 31.5 & W \\
\hline 887.1 & carcar & 17.4 & w \\
\hline 899.3 & carcar & 14.0 & $\mathrm{w}$ \\
\hline 900.8 & nyssyl & 34.8 & $\mathrm{w}$ \\
\hline 903.1 & carcar & 18.4 & $\mathrm{w}$ \\
\hline 905.9 & nyssyl & 19.5 & $\mathrm{w}$ \\
\hline 906.1 & carcar & 18.2 & $\mathrm{w}$ \\
\hline 916.6 & nysbif & 23.4 & w \\
\hline 916.6 & \multicolumn{3}{|c|}{ marker \# 51} \\
\hline
\end{tabular}


C FK: The sampling width of the west segment is 10 meters, 5 meters on either side of the transect line. The sampling width of the east segment was 5 meters on the south side of the transect only

\begin{tabular}{|c|c|c|c|c|c|c|c|}
\hline $\begin{array}{l}\text { Distance from } \\
\text { beginning of } \\
\text { segment in } \mathrm{m}\end{array}$ & $\begin{array}{c}\text { Tree } \\
\text { species }\end{array}$ & $\begin{array}{l}\text { Dbh, } \\
\text { in cm }\end{array}$ & Side & $\begin{array}{l}\text { Distance from } \\
\text { beginning of } \\
\text { segment in } \mathrm{m}\end{array}$ & $\begin{array}{c}\text { Tree } \\
\text { species }\end{array}$ & $\begin{array}{l}\text { Dbh, } \\
\text { in cm }\end{array}$ & Side \\
\hline \multicolumn{4}{|c|}{ East segment } & 115.5 & fracar & 18.2 & $\mathrm{~s}$ \\
\hline \multirow{2}{*}{-9.5} & \multirow{2}{*}{ fracar } & 10.2 & \multirow{2}{*}{$\mathrm{s}$} & 115.9 & fracar & 13.4 & $\mathrm{~s}$ \\
\hline & & 8.0 & & 117.2 & taxdis & 32.3 & $\mathrm{~s}$ \\
\hline \multirow{3}{*}{-8.5} & \multirow{3}{*}{ fracar } & 12.8 & \multirow{3}{*}{$\mathrm{s}$} & 118.7 & taxdis & 25.2 & $\mathrm{~s}$ \\
\hline & & 5.9 & & 120.3 & quelyr & 59.5 & $\mathrm{~s}$ \\
\hline & & 10.3 & & 133.2 & fracar & 15.4 & $\mathrm{~s}$ \\
\hline-8.5 & snag & 54.0 & $\mathrm{~s}$ & 133.7 & fracar & 18.9 & $\mathrm{~s}$ \\
\hline-8.0 & fracar & 11.9 & $\mathrm{~s}$ & 138.7 & fracar & 14.7 & $\mathrm{~s}$ \\
\hline-8.0 & fracar & 10.6 & $\mathrm{~s}$ & 138.7 & fracar & 13.6 & $\mathrm{~s}$ \\
\hline \multirow{2}{*}{-7.9} & \multirow{2}{*}{ fracar } & 16.2 & \multirow{2}{*}{$\mathrm{s}$} & 138.8 & fracar & 10.4 & $\mathrm{~s}$ \\
\hline & & 4.1 & & \multirow{2}{*}{138.9} & \multirow{2}{*}{ fracar } & 14.5 & \multirow{2}{*}{$\mathrm{s}$} \\
\hline-3.4 & quenig & 35.9 & $\mathrm{~s}$ & & & 14.4 & \\
\hline 0.0 & \multicolumn{3}{|c|}{ marker \# 52} & 138.9 & fracar & 17.6 & $\mathrm{~s}$ \\
\hline 11.8 & quenig & 11.6 & $\mathrm{~s}$ & 138.9 & fracar & 4.4 & $\mathrm{~s}$ \\
\hline 12.2 & liqsty & 29.6 & $\mathrm{~s}$ & 139.0 & fracar & 10.3 & $\mathrm{~s}$ \\
\hline 19.2 & carcar & 11.0 & $\mathrm{~s}$ & 139.2 & snag & 15.1 & $\mathrm{~s}$ \\
\hline 20.0 & liqsty & 37.4 & $\mathrm{~s}$ & 142.4 & cepocc & 12.0 & $\mathrm{~s}$ \\
\hline 22.9 & carcar & 10.9 & $\mathrm{~s}$ & 144.1 & \multicolumn{3}{|c|}{ marker \# 56} \\
\hline 32.7 & quenig & 32.3 & $\mathrm{~s}$ & \multirow{2}{*}{146.0} & \multirow{2}{*}{ acerub } & 18.9 & \multirow{2}{*}{$\mathrm{s}$} \\
\hline 34.7 & betnig & 28.3 & $\mathrm{~s}$ & & & 12.1 & \\
\hline 37.1 & carcar & 13.5 & $\mathrm{~s}$ & 146.3 & plaaqu & 20.1 & $\mathrm{~s}$ \\
\hline 37.5 & vibobo & 11.4 & $\mathrm{~s}$ & 152.4 & ulmame & 16.4 & $\mathrm{~s}$ \\
\hline 38.3 & carcar & 10.3 & $\mathrm{~s}$ & 154.5 & acerub & 26.4 & $\mathrm{~s}$ \\
\hline 40.0 & \multicolumn{3}{|c|}{ marker \# 53} & 156.4 & acerub & 25.8 & $\mathrm{~s}$ \\
\hline \multirow{2}{*}{40.5} & \multirow{2}{*}{ carcar } & 13.7 & \multirow{2}{*}{$\mathrm{s}$} & 156.4 & gleaqu & 30.4 & $\mathrm{~s}$ \\
\hline & & 9.4 & & 158.2 & acerub & 10.5 & $\mathrm{~s}$ \\
\hline 43.3 & carcar & 13.2 & $\mathrm{~s}$ & 158.5 & ulmame & 10.4 & $\mathrm{~s}$ \\
\hline 48.6 & betnig & 15.3 & $\mathrm{~s}$ & 159.0 & taxdis & 38.4 & $\mathrm{~s}$ \\
\hline 49.7 & carcar & 16.0 & $\mathrm{~s}$ & 161.8 & ulmame & 10.6 & $\mathrm{~s}$ \\
\hline 53.5 & caraqu & 57.3 & $\mathrm{~s}$ & 165.9 & taxdis & 43.0 & $\mathrm{~s}$ \\
\hline 61.7 & gleaqu & 33.5 & $\mathrm{~s}$ & 180.6 & acerub & 18.9 & $\mathrm{~s}$ \\
\hline 70.7 & taxdis & 56.0 & $\mathrm{~s}$ & 182.6 & taxdis & 56.0 & $\mathrm{~s}$ \\
\hline 72.0 & & $\# 54$ & & 183.1 & acerub & 21.7 & $\mathrm{~s}$ \\
\hline 74.5 & taxdis & 59.4 & $\mathrm{~s}$ & 185.3 & & $\# 57$ & \\
\hline 750 & fracar & 12.6 & $\mathrm{~s}$ & 186.9 & snag & 14.2 & $\mathrm{~s}$ \\
\hline 10. & 11 acal & 5.9 & $\mathrm{~s}$ & 189.7 & plaaqu & 26.0 & $\mathrm{~s}$ \\
\hline 78.7 & betnig & 11.4 & $\mathrm{~s}$ & & & 13.9 & \\
\hline 90.4 & plaaqu & 45.9 & $\mathrm{~s}$ & & & 8.6 & \\
\hline 98.8 & taxdis & 25.5 & $\mathrm{~s}$ & 194.7 & fracar & 8.0 & $\mathrm{~s}$ \\
\hline 103.6 & & $\# 55$ & & & & 9.0 & \\
\hline 104.5 & taxdis & 53.8 & $\mathrm{~s}$ & & & 9.0 & \\
\hline 105.7 & fracar & 13.9 & $\mathrm{~s}$ & & & 11.3 & \\
\hline 113.5 & fracar & 13.3 & $\mathrm{~s}$ & 200.3 & fracar & 9.9 & $\mathrm{~s}$ \\
\hline 113.6 & fracar & 13.7 & $\mathrm{~s}$ & & & 4.0 & \\
\hline 115.3 & fracar & 19.9 & $\mathrm{~s}$ & & & & \\
\hline
\end{tabular}


C FK: The sampling width of the west segment is 10 meters, 5 meters on either side of the transect line. The sampling width of the east segment was 5 meters on the south side of the transect only (Continued)

\begin{tabular}{|c|c|c|c|c|c|c|c|}
\hline $\begin{array}{l}\text { Distance from } \\
\text { beginning of } \\
\text { segment in } \mathrm{m}\end{array}$ & $\begin{array}{c}\text { Tree } \\
\text { species }\end{array}$ & $\begin{array}{l}\text { Dbh, } \\
\text { in cm }\end{array}$ & Side & $\begin{array}{l}\text { Distance from } \\
\text { beginning of } \\
\text { segment in } \mathrm{m}\end{array}$ & $\begin{array}{c}\text { Tree } \\
\text { species }\end{array}$ & $\begin{array}{l}\text { Dbh, } \\
\text { in } \mathrm{cm}\end{array}$ & Side \\
\hline \multirow{3}{*}{201.4} & \multirow{3}{*}{ acerub } & 26.4 & \multirow{3}{*}{$\mathrm{s}$} & \multirow{2}{*}{31.7} & \multirow{2}{*}{ cepocc } & 11.9 & \multirow{2}{*}{$\mathrm{s}$} \\
\hline & & 24.9 & & & & 7.0 & \\
\hline & & 11.5 & & 32.7 & nysaqu & 41.5 & $\mathrm{~s}$ \\
\hline 203.4 & taxdis & 40.7 & $\mathrm{~s}$ & 33.4 & taxdis & 23.9 & $\mathrm{n}$ \\
\hline 204.1 & acerub & 16.6 & $\mathrm{~s}$ & \multirow{3}{*}{34.7} & \multirow{3}{*}{ fracar } & 10.7 & \multirow{3}{*}{$\mathrm{n}$} \\
\hline \multirow{5}{*}{204.3} & \multirow{5}{*}{ fracar } & 15.7 & \multirow{5}{*}{$\mathrm{s}$} & & & 4.6 & \\
\hline & & 7.6 & & & & 8.5 & \\
\hline & & 11.0 & & 35.5 & nysaqu & 40.6 & $\mathrm{~s}$ \\
\hline & & 15.4 & & 36.5 & cepocc & 10.3 & $\mathrm{~s}$ \\
\hline & & 7.2 & & 39.0 & cepocc & 10.4 & $\mathrm{n}$ \\
\hline 205.0 & plaaqu & 12.6 & $\mathrm{~s}$ & 39.1 & cepocc & 15.3 & $\mathrm{~s}$ \\
\hline 210.3 & \multicolumn{3}{|c|}{ marker \# 58} & 39.3 & salcar & 29.4 & $\mathrm{n}$ \\
\hline \multicolumn{4}{|c|}{ West segment } & 40.3 & cepocc & 13.4 & $\mathrm{~s}$ \\
\hline 0.0 & \multicolumn{3}{|c|}{ marker \# 66} & 40.9 & salcar & 25.9 & $\mathrm{~s}$ \\
\hline \multirow{5}{*}{0.6} & \multirow{5}{*}{ betnig } & 10.0 & & \multirow{2}{*}{42.2} & \multirow{2}{*}{ fracar } & 8.4 & \multirow{2}{*}{$\mathrm{n}$} \\
\hline & & 10.4 & & & & 16.3 & \\
\hline & & 8.1 & $\mathrm{n}$ & 42.2 & taxdis & 11.4 & $\mathrm{n}$ \\
\hline & & 5.4 & & 42.2 & fracar & 10.3 & $\mathrm{~s}$ \\
\hline & & 5.7 & & 42.6 & taxdis & 18.3 & $\mathrm{n}$ \\
\hline 7.8 & taxdis & 35.0 & $\mathrm{~S}$ & 42.7 & taxdis & 17.5 & $\mathrm{~s}$ \\
\hline 8.3 & betnig & 12.0 & $\mathrm{~s}$ & 43.2 & cepocc & 11.5 & $\mathrm{~s}$ \\
\hline 9.5 & betnig & 13.9 & $\mathrm{n}$ & 44.6 & taxdis & 17.3 & $\mathrm{~s}$ \\
\hline 14.0 & acerub & 11.9 & $\mathrm{~s}$ & 45.4 & cepocc & 11.9 & $\mathrm{n}$ \\
\hline \multirow{4}{*}{14.1} & \multirow{4}{*}{ acerub } & 11.3 & & 45.6 & taxdis & 20.0 & $\mathrm{~s}$ \\
\hline & & 4.5 & $\mathrm{~S}$ & 45.7 & taxdis & 14.3 & $\mathrm{~s}$ \\
\hline & & 9.0 & s & 46.4 & salcar & 16.4 & $\mathrm{n}$ \\
\hline & & 6.5 & & 47.6 & & \# 64 & \\
\hline 14.3 & acerub & 21.6 & $\mathrm{n}$ & 47.9 & fracar & 10.5 & $\mathrm{~s}$ \\
\hline 14.6 & snag & 30.7 & $\mathrm{n}$ & 48.1 & fracar & 10.3 & $\mathrm{~s}$ \\
\hline 14.6 & acerub & 16.9 & $\mathrm{~s}$ & & & 13.4 & \\
\hline 14.9 & betnig & 14.5 & $\mathrm{~s}$ & 49.2 & fracar & 8.5 & $\mathrm{~s}$ \\
\hline 15.6 & taxdis & 24.4 & $\mathrm{n}$ & & & 4.5 & \\
\hline 18.6 & nysaqu & 24.9 & $\mathrm{n}$ & & & 10.8 & \\
\hline 19.0 & betnig & 10.2 & $\mathrm{n}$ & & & 9.0 & \\
\hline 19.2 & taxdis & 40.2 & $\mathrm{n}$ & 49.5 & fracar & 8.0 & $\mathrm{~s}$ \\
\hline 20.3 & taxdis & 20.1 & $\mathrm{n}$ & & & 5.0 & \\
\hline 21.4 & taxdis & 35.9 & $\mathrm{n}$ & & & 4.8 & \\
\hline 23.7 & nysaqu & 42.4 & $\mathrm{~s}$ & & & 10.7 & \\
\hline 25.9 & taxdis & 36.1 & $\mathrm{~s}$ & 504 & fropos & 8.8 & \\
\hline 26.1 & & $\# 65$ & & 50.4 & fracar & 7.4 & $\mathrm{n}$ \\
\hline 26.1 & nysaqu & 49.2 & $\mathrm{n}$ & & & 7.5 & \\
\hline 26.2 & snag & 23.3 & $\mathrm{n}$ & 50.6 & fracar & 11.8 & $\mathrm{n}$ \\
\hline 27.2 & nysaqu & 24.2 & $\mathrm{n}$ & 52.0 & salcar & 30.3 & $\mathrm{~s}$ \\
\hline 27.7 & taxdis & 40.0 & $\mathrm{~s}$ & 523 & toudi & 28.3 & \\
\hline 29.7 & nysaqu & 46.3 & $\mathrm{n}$ & 53.3 & taxais & 47.4 & S \\
\hline 31.2 & cepocc & 11.5 & $\mathrm{n}$ & 53.8 & betnig & 10.2 & $\mathrm{n}$ \\
\hline
\end{tabular}


C FK: The sampling width of the west segment is 10 meters, 5 meters on either side of the transect line. The sampling width of the east segment was 5 meters on the south side of the transect only (Continued)

\begin{tabular}{|c|c|c|c|c|c|c|c|}
\hline $\begin{array}{l}\text { Distance from } \\
\text { beginning of } \\
\text { segment in } \mathrm{m}\end{array}$ & $\begin{array}{c}\text { Tree } \\
\text { species }\end{array}$ & $\begin{array}{l}\text { Dbh, } \\
\text { in cm }\end{array}$ & Side & $\begin{array}{l}\text { Distance from } \\
\text { beginning of } \\
\text { segment in } \mathrm{m}\end{array}$ & $\begin{array}{c}\text { Tree } \\
\text { species }\end{array}$ & $\begin{array}{l}\text { Dbh, } \\
\text { in cm }\end{array}$ & Side \\
\hline 53.9 & taxdis & 45.0 & $\mathrm{~s}$ & \multirow{6}{*}{97.5} & \multirow{6}{*}{ fracar } & 10.0 & \multirow{6}{*}{$\mathrm{s}$} \\
\hline 54.1 & cepocc & 10.7 & $\mathrm{n}$ & & & 7.0 & \\
\hline \multirow{4}{*}{55.7} & \multirow{4}{*}{ fracar } & 6.5 & \multirow{4}{*}{$\mathrm{n}$} & & & 9.9 & \\
\hline & & 12.3 & & & & 7.0 & \\
\hline & & 8.2 & & & & 4.5 & \\
\hline & & 6.2 & & & & 7.0 & \\
\hline 56.4 & salcar & 24.6 & $\mathrm{~s}$ & 97.6 & fracar & 16.8 & $\mathrm{n}$ \\
\hline 59.1 & acerub & 26.5 & $\mathrm{~s}$ & 97.6 & plaaqu & 35.9 & $\mathrm{n}$ \\
\hline 59.1 & salcar & 28.5 & $\mathrm{~s}$ & \multirow{2}{*}{99.9} & \multirow{2}{*}{ fracar } & 14.1 & \multirow{2}{*}{$\mathrm{n}$} \\
\hline 60.0 & salcar & 27.7 & $\mathrm{n}$ & & & 4.8 & \\
\hline 62.4 & cepocc & 11.6 & $\mathrm{~s}$ & 100.0 & fracar & 10.2 & $\mathrm{n}$ \\
\hline 63.3 & nysaqu & 55.0 & $\mathrm{~s}$ & 100.2 & taxdis & 53.9 & $\mathrm{~s}$ \\
\hline 68.1 & \multicolumn{3}{|c|}{ marker \# 63} & 101.2 & \multicolumn{3}{|c|}{ marker \# 62} \\
\hline 68.7 & taxdis & 36.1 & $\mathrm{~s}$ & 103.1 & nysaqu & 26.8 & $\mathrm{~s}$ \\
\hline 69.4 & cepocc & 14.1 & $\mathrm{n}$ & 103.6 & salcar & 14.4 & $\mathrm{~s}$ \\
\hline 69.6 & taxdis & 44.5 & $\mathrm{~s}$ & 103.6 & taxdis & 48.5 & $\mathrm{~s}$ \\
\hline 70.3 & taxdis & 50.4 & $\mathrm{n}$ & \multirow{3}{*}{104.6} & \multirow{3}{*}{ fracar } & 8.0 & \multirow{3}{*}{$\mathrm{n}$} \\
\hline 71.3 & betnig & 13.1 & $\mathrm{n}$ & & & 12.6 & \\
\hline 72.5 & taxdis & 32.9 & $\mathrm{n}$ & & & 5.5 & \\
\hline 72.8 & taxdis & 36.9 & $\mathrm{n}$ & 105.0 & taxdis & 42.4 & $\mathrm{~s}$ \\
\hline 73.2 & taxdis & 45.0 & $\mathrm{n}$ & 105.2 & nysaqu & 29.4 & $\mathrm{~s}$ \\
\hline 73.2 & snag & 15.5 & $\mathrm{~s}$ & 106.2 & cepocc & 12.5 & $\mathrm{~s}$ \\
\hline 73.5 & nysaqu & 26.4 & $\mathrm{~s}$ & 106.7 & fracar & 13.3 & $\mathrm{n}$ \\
\hline 73.8 & fracar & 10.6 & $\mathrm{n}$ & 106.9 & fracar & 11.2 & $\mathrm{n}$ \\
\hline 77.2 & taxdis & 33.0 & $\mathrm{~s}$ & 106.9 & fracar & 10.6 & $\mathrm{n}$ \\
\hline 77.3 & taxdis & 28.1 & $\mathrm{n}$ & 109.4 & salcar & 19.5 & $\mathrm{~s}$ \\
\hline \multirow{2}{*}{77.8} & \multirow{2}{*}{ cepocc } & 10.8 & \multirow{2}{*}{$\mathrm{n}$} & 109.4 & salcar & 30.0 & $\mathrm{~s}$ \\
\hline & & 11.0 & & \multirow{3}{*}{109.7} & \multirow{3}{*}{ fracar } & 11.9 & \multirow{3}{*}{$\mathrm{n}$} \\
\hline 78.6 & taxdis & 43.2 & $\mathrm{n}$ & & & 7.0 & \\
\hline 79.9 & betnig & 10.8 & $\mathrm{~s}$ & & & 7.4 & \\
\hline 80.6 & nysaqu & 87.4 & $\mathrm{~s}$ & 110.4 & fracar & 14.7 & $\mathrm{n}$ \\
\hline 81.7 & salcar & 22.4 & $\mathrm{n}$ & 110.4 & fracar & 8.9 & $\mathrm{n}$ \\
\hline 84.4 & salcar & 19.0 & $\mathrm{n}$ & 111.1 & gleaqu & 16.2 & $\mathrm{n}$ \\
\hline 87.0 & taxdis & 11.0 & $\mathrm{n}$ & 111.5 & cepocc & 13.4 & $\mathrm{n}$ \\
\hline 88.9 & cepocc & 14.4 & $\mathrm{n}$ & 113.2 & taxdis & 54.0 & $\mathrm{~s}$ \\
\hline 89.0 & acerub & 51.0 & $\mathrm{n}$ & 114.9 & fracar & 10.2 & $\mathrm{~s}$ \\
\hline \multirow{3}{*}{89.0} & \multirow{3}{*}{ fracar } & 10.6 & & 115.0 & fracar & 10.5 & $\mathrm{~s}$ \\
\hline & & 6.9 & $\mathrm{n}$ & $116 ?$ & fracar & 16.1 & $n$ \\
\hline & & 5.8 & & 110.2 & Iracar & 10.9 & $\mathrm{n}$ \\
\hline 91.9 & nysaqu & 32.5 & $\mathrm{~s}$ & 1160 & & 11.3 & \\
\hline 92.0 & taxdis & 110.0 & $\mathrm{n}$ & 116.9 & серосc & 10.6 & $\mathrm{~s}$ \\
\hline 93.1 & nysaqu & 12.2 & $\mathrm{~s}$ & 117.2 & snag & 10.0 & $\mathrm{~s}$ \\
\hline 93.8 & nysaqu & 26.6 & $\mathrm{~s}$ & 117.6 & & $\# 61$ & \\
\hline 048 & & 14.7 & & & & 11.3 & \\
\hline 94.8 & fracar & 5.0 & $\mathrm{n}$ & 118.2 & fracar & 13.8 & $\mathrm{~s}$ \\
\hline & & & & & & 13.3 & \\
\hline
\end{tabular}


C FK: The sampling width of the west segment is 10 meters, 5 meters on either side of the transect line. The sampling width of the east segment was 5 meters on the south side of the transect only (Continued)

\begin{tabular}{|c|c|c|c|c|c|c|c|}
\hline $\begin{array}{l}\text { Distance from } \\
\text { beginning of } \\
\text { segment in } \mathrm{m}\end{array}$ & $\begin{array}{c}\text { Tree } \\
\text { species }\end{array}$ & $\begin{array}{l}\text { Dbh, } \\
\text { in cm }\end{array}$ & Side & $\begin{array}{l}\text { Distance from } \\
\text { beginning of } \\
\text { segment in } \mathrm{m}\end{array}$ & $\begin{array}{c}\text { Tree } \\
\text { species }\end{array}$ & $\begin{array}{l}\text { Dbh, } \\
\text { in cm }\end{array}$ & Side \\
\hline 119.2 & cepocc & 11.8 & $\mathrm{~S}$ & \multirow{3}{*}{139.3} & \multirow{3}{*}{ fracar } & 10.5 & \multirow{3}{*}{$\mathrm{S}$} \\
\hline \multirow{4}{*}{119.5} & \multirow{4}{*}{ fracar } & 12.9 & \multirow{4}{*}{$\mathrm{s}$} & & & 7.0 & \\
\hline & & 6.3 & & & & 5.7 & \\
\hline & & 7.5 & & \multirow{3}{*}{140.0} & \multirow{3}{*}{ fracar } & 11.5 & \multirow{3}{*}{$\mathrm{s}$} \\
\hline & & 6.7 & & & & 7.5 & \\
\hline \multirow{2}{*}{120.9} & \multirow{2}{*}{ fracar } & 15.3 & \multirow{2}{*}{$\mathrm{n}$} & & & 7.2 & \\
\hline & & 7.0 & & 140.1 & fracar & 13.4 & $\mathrm{~s}$ \\
\hline \multirow{2}{*}{121.9} & \multirow{2}{*}{ fracar } & 10.1 & \multirow{2}{*}{$\mathrm{n}$} & 140.2 & fracar & 11.5 & $\mathrm{~s}$ \\
\hline & & 6.7 & & 141.0 & fracar & 13.7 & $\mathrm{~s}$ \\
\hline 122.8 & fracar & 16.3 & $\mathrm{n}$ & 141.7 & salcar & 23.2 & $\mathrm{~s}$ \\
\hline 123.7 & ulmame & 18.8 & $\mathrm{~s}$ & 141.8 & ulmame & 20.9 & $\mathrm{~s}$ \\
\hline 124.4 & fracar & 13.5 & $\mathrm{n}$ & 142.1 & fracar & 10.9 & $\mathrm{n}$ \\
\hline 124.6 & taxdis & 23.8 & $\mathrm{~s}$ & \multirow{2}{*}{142.1} & \multirow{2}{*}{ fracar } & 10.3 & \multirow{2}{*}{$\mathrm{n}$} \\
\hline 125.9 & cepocc & 12.6 & $\mathrm{n}$ & & & 9.8 & \\
\hline \multirow{3}{*}{125.9} & \multirow{3}{*}{ cepocc } & 10.0 & \multirow{3}{*}{$\mathrm{S}$} & 142.8 & cepocc & 12.3 & $\mathrm{n}$ \\
\hline & & 5.5 & & 143.6 & cepocc & 13.2 & $\mathrm{n}$ \\
\hline & & 7.3 & & 145.1 & cepocc & 12.0 & $\mathrm{~s}$ \\
\hline 127.5 & taxdis & 30.8 & $\mathrm{n}$ & 146.6 & nysaqu & 24.9 & $\mathrm{~s}$ \\
\hline 129.5 & cepocc & 10.1 & $\mathrm{n}$ & 146.6 & nysaqu & 46.5 & $\mathrm{~s}$ \\
\hline 129.7 & fracar & 11.4 & $\mathrm{n}$ & 146.9 & nysaqu & 30.4 & $\mathrm{~s}$ \\
\hline \multirow{4}{*}{129.8} & & 4.6 & & 147.2 & nysaqu & 29.7 & $\mathrm{~s}$ \\
\hline & frocor & 22.4 & $n$ & 147.6 & nysaqu & 33.0 & $\mathrm{n}$ \\
\hline & $11 \mathrm{ac}$ al & 11.8 & 11 & 1470 & fract & 12.3 & $\mathrm{~s}$ \\
\hline & & 10.5 & & 141.5 & II acd & 9.0 & $\mathrm{~s}$ \\
\hline 129.9 & fracar & 14.9 & $\mathrm{~s}$ & 148.2 & fracar & 10.7 & $\mathrm{n}$ \\
\hline 1301 & cenncs & 10.5 & $\mathrm{~s}$ & 148.6 & cepocc & 10.0 & $\mathrm{n}$ \\
\hline 150.1 & eерое & 5.6 & $\mathrm{~s}$ & 149.1 & nysaqu & 33.3 & $\mathrm{n}$ \\
\hline 131.0 & fracar & 13.2 & $\mathrm{~s}$ & 149.3 & fracar & 12.3 & $\mathrm{n}$ \\
\hline & & 10.5 & & 151.3 & & \# 59 & \\
\hline 1210 & f & 9.0 & & & & & \\
\hline 151.0 & Iracal & 7.3 & $\mathrm{~s}$ & & & & \\
\hline & & 10.1 & & & & & \\
\hline 1321 & fracar & 12.0 & $\mathrm{n}$ & & & & \\
\hline 152.1 & Iracar & 4.5 & $\mathrm{n}$ & & & & \\
\hline 132.9 & salcar & 23.3 & $\mathrm{~s}$ & & & & \\
\hline 133.3 & fracar & 13.3 & $\mathrm{n}$ & & & & \\
\hline 134.0 & cepocc & 12.8 & $\mathrm{~s}$ & & & & \\
\hline 134.1 & cepocc & 11.5 & $\mathrm{~s}$ & & & & \\
\hline 134.2 & snag & 11.4 & $\mathrm{n}$ & & & & \\
\hline & & 17.5 & & & & & \\
\hline 135.1 & fracar & 9.4 & $\mathrm{~s}$ & & & & \\
\hline & & 9.4 & & & & & \\
\hline 1371 & & 14.6 & & & & & \\
\hline $13 / .1$ & cеросc & 7.3 & $\mathrm{~S}$ & & & & \\
\hline 138.8 & & $\# 60$ & & & & & \\
\hline
\end{tabular}


D MS: The sampling width of this transect is 10 meters (5 meters on each side of the transect line) from $5.3 \mathrm{~m}$ to $413 \mathrm{~m}$ and from $825.6 \mathrm{~m}$ to $902.2 \mathrm{~m}$. The sampling width is 5 meters on the west side of the transect line from 413 to 825.6 and from 902.2 to 1003.7

\begin{tabular}{|c|c|c|c|c|c|c|c|}
\hline $\begin{array}{l}\text { Distance from } \\
\text { beginning of } \\
\text { transect, in } \mathrm{m}\end{array}$ & $\begin{array}{c}\text { Tree } \\
\text { species }\end{array}$ & $\begin{array}{l}\text { Dbh, } \\
\text { in cm }\end{array}$ & Side & $\begin{array}{l}\text { Distance from } \\
\text { beginning of } \\
\text { transect, in } \mathrm{m}\end{array}$ & $\begin{array}{c}\text { Tree } \\
\text { species }\end{array}$ & $\begin{array}{l}\text { Dbh, } \\
\text { in cm }\end{array}$ & Side \\
\hline 0.0 & \multicolumn{3}{|c|}{ marker \# 67} & 128.7 & snag & 22.5 & $\mathrm{e}$ \\
\hline \multirow{3}{*}{15.9} & \multirow{3}{*}{ acerub } & 30.5 & \multirow{3}{*}{$\mathrm{w}$} & 131.3 & carcar & 10.1 & w \\
\hline & & 41.0 & & 135.1 & carcar & 13.5 & $\mathrm{e}$ \\
\hline & & 13.0 & & 140.5 & nyssyl & 24.6 & $\mathrm{e}$ \\
\hline 17.5 & snag & 19.0 & w & 140.9 & carcar & 10.2 & w \\
\hline 18.3 & carcar & 12.4 & w & 146.3 & carcar & 10.7 & $\mathrm{w}$ \\
\hline 25.6 & liqsty & 22.1 & $\mathrm{w}$ & 148.5 & carcar & 12.1 & w \\
\hline 30.8 & liqsty & 17.0 & $\mathrm{w}$ & 150.5 & \multicolumn{3}{|c|}{ marker \# 70} \\
\hline 31.4 & liqsty & 16.6 & w & 150.6 & snag & 81.0 & $\mathrm{e}$ \\
\hline 31.9 & liqsty & 15.8 & $\mathrm{w}$ & 152.3 & liqsty & 16.1 & $\mathrm{e}$ \\
\hline 39.6 & liqsty & 14.5 & $\mathrm{w}$ & 156.3 & carcar & 14.0 & w \\
\hline 42.5 & liqsty & 18.4 & $\mathrm{w}$ & 156.7 & quenig & 42.8 & w \\
\hline 47.8 & quelau & 42.2 & $\mathrm{w}$ & \multirow{2}{*}{164.7} & \multirow{2}{*}{ carcar } & 19.2 & \multirow{2}{*}{$\mathrm{w}$} \\
\hline 48.1 & acerub & 32.3 & $\mathrm{w}$ & & & 4.5 & \\
\hline 48.2 & \multicolumn{3}{|c|}{ marker \# 68} & \multirow{3}{*}{165.0} & \multirow{3}{*}{ carcar } & 22.8 & \multirow{3}{*}{$\mathrm{e}$} \\
\hline 49.1 & quelau & 25.3 & $\mathrm{w}$ & & & 7.7 & \\
\hline 50.5 & acerub & 10.4 & $\mathrm{w}$ & & & 5.5 & \\
\hline 60.7 & carcar & 11.9 & w & \multirow{2}{*}{170.5} & \multirow{2}{*}{ carcar } & 16.6 & \multirow{2}{*}{$\mathrm{e}$} \\
\hline 64.6 & acerub & 28.9 & w & & & 10.3 & \\
\hline 69.7 & liqsty & 13.9 & $\mathrm{w}$ & 172.7 & carcar & 15.4 & $\mathrm{w}$ \\
\hline 73.3 & quelau & 23.9 & $\mathrm{w}$ & 174.8 & carcar & 12.1 & $\mathrm{e}$ \\
\hline 75.4 & quelau & 39.9 & w & 185.4 & carcar & 10.4 & w \\
\hline 80.4 & carcar & 13.0 & $\mathrm{w}$ & 189.4 & carcar & 12.1 & $\mathrm{w}$ \\
\hline 80.6 & quelau & 38.7 & $\mathrm{w}$ & 200.0 & \multicolumn{3}{|c|}{ marker \# 71} \\
\hline 82.2 & quelau & 44.8 & $\mathrm{w}$ & 200.2 & carcar & 19.3 & w \\
\hline 84.2 & liqsty & 11.8 & $\mathrm{w}$ & 201.7 & snag & 22.0 & $\mathrm{w}$ \\
\hline 87.8 & quelau & 52.5 & w & 207.8 & carcar & 10.6 & $\mathrm{w}$ \\
\hline 91.1 & liqsty & 16.9 & $\mathrm{w}$ & 208.2 & quevir & 35.4 & w \\
\hline 94.5 & liqsty & 11.4 & w & 211.4 & betnig & 40.8 & w \\
\hline 96.6 & liqsty & 17.0 & $\mathrm{w}$ & 213.3 & taxdis & 16.6 & w \\
\hline 102.2 & liqsty & 14.8 & $\mathrm{w}$ & 213.3 & taxdis & 13.6 & w \\
\hline 102.6 & \multicolumn{3}{|c|}{ marker \# 69} & 215.2 & taxdis & 32.5 & $\mathrm{w}$ \\
\hline 105.4 & liqsty & 13.5 & $\mathrm{e}$ & 215.8 & taxdis & 30.0 & $\mathrm{w}$ \\
\hline 106.8 & liqsty & 19.3 & w & 217.3 & taxdis & 19.4 & $\mathrm{w}$ \\
\hline 108.4 & carcar & 20.8 & w & \multirow{3}{*}{218.1} & \multirow{3}{*}{ acerub } & 26.4 & \multirow{3}{*}{ w } \\
\hline 110.1 & liqsty & 18.2 & $\mathrm{e}$ & & & 8.4 & \\
\hline 112.9 & carcar & 12.7 & $\mathrm{e}$ & & & 8.2 & \\
\hline 120.0 & carcar & 17.2 & $\mathrm{w}$ & 219.4 & taxdis & 24.6 & $\mathrm{w}$ \\
\hline 121.2 & quevir & 35.4 & w & 220.3 & ulmame & 32.3 & w \\
\hline 122.8 & carcar & 10.5 & $\mathrm{w}$ & 223.1 & taxdis & 49.1 & $\mathrm{w}$ \\
\hline 124.0 & quelau & 26.1 & $\mathrm{e}$ & 224.2 & nysaqu & 28.8 & w \\
\hline 127.0 & liqsty & 18.3 & $\mathrm{w}$ & 228.7 & plaaqu & 11.6 & $\mathrm{w}$ \\
\hline
\end{tabular}


D MS: The sampling width of this transect is 10 meters ( 5 meters on each side of the transect line) from $5.3 \mathrm{~m}$ to $413 \mathrm{~m}$ and from $825.6 \mathrm{~m}$ to $902.2 \mathrm{~m}$. The sampling width is 5 meters on the west side of the transect line from 413 to 825.6 and from 902.2 to 1003.7 (Continued)

\begin{tabular}{|c|c|c|c|c|c|c|c|}
\hline $\begin{array}{l}\text { Distance from } \\
\text { beginning of } \\
\text { transect, in } m\end{array}$ & $\begin{array}{c}\text { Tree } \\
\text { species }\end{array}$ & $\begin{array}{l}\text { Dbh, } \\
\text { in cm }\end{array}$ & Side & $\begin{array}{l}\text { Distance from } \\
\text { beginning of } \\
\text { transect, in } \mathrm{m}\end{array}$ & $\begin{array}{c}\text { Tree } \\
\text { species }\end{array}$ & $\begin{array}{l}\text { Dbh, } \\
\text { in } \mathrm{cm}\end{array}$ & Side \\
\hline 229.3 & nysaqu & 25.6 & $\mathrm{~W}$ & 293.3 & fracar & 11.9 & $\mathrm{~W}$ \\
\hline \multirow{5}{*}{229.5} & \multirow{5}{*}{ fracar } & 6.0 & \multirow{5}{*}{$\mathrm{w}$} & 293.9 & fracar & 13.6 & $\mathrm{w}$ \\
\hline & & 12.3 & & 296.0 & fracar & 10.2 & w \\
\hline & & 9.9 & & 296.9 & fracar & 10.1 & $\mathrm{w}$ \\
\hline & & 7.0 & & \multirow{2}{*}{297.3} & \multirow{2}{*}{ fracar } & 10.7 & \multirow{2}{*}{$\mathrm{w}$} \\
\hline & & 9.9 & & & & 7.4 & \\
\hline 238.1 & \multicolumn{3}{|c|}{ marker \# 72} & 299.0 & nysaqu & 34.7 & $\mathrm{w}$ \\
\hline 240.8 & nysaqu & 43.2 & $\mathrm{w}$ & 304.3 & nysaqu & 35.9 & $\mathrm{w}$ \\
\hline \multirow{2}{*}{244.3} & \multirow{2}{*}{ fracar } & 14.0 & \multirow{2}{*}{$\mathrm{W}$} & \multirow{3}{*}{306.2} & \multirow{3}{*}{ plaaqu } & 35.0 & \multirow{3}{*}{$\mathrm{w}$} \\
\hline & & 6.8 & & & & 23.6 & \\
\hline 246.8 & nysaqu & 33.4 & $\mathrm{~W}$ & & & 10.0 & \\
\hline \multirow{2}{*}{247.1} & \multirow{2}{*}{ nysaqu } & 33.9 & \multirow{2}{*}{$\mathrm{W}$} & \multirow{2}{*}{309.5} & \multirow{2}{*}{ fracar } & 15.2 & \multirow{2}{*}{$\mathrm{w}$} \\
\hline & & 36.2 & & & & 10.6 & \\
\hline 247.7 & fracar & 10.1 & w & \multirow{2}{*}{309.8} & \multirow{2}{*}{ fracar } & 12.9 & w \\
\hline & & 10.4 & & & & 8.6 & W \\
\hline 252.3 & серосс & 4.6 & W & 312.1 & fracar & 10.8 & w \\
\hline & & 12.6 & & 312.2 & fracar & 11.2 & $\mathrm{w}$ \\
\hline 252.3 & fracar & 11.2 & $\mathrm{w}$ & 3128 & plag & 20.4 & \\
\hline & & 7.0 & & 312.8 & plaaqu & 13.1 & W \\
\hline 253.2 & betnig & 18.0 & $\mathrm{w}$ & 313.6 & plaaqu & 20.6 & $\mathrm{w}$ \\
\hline 255.2 & betnig & 26.7 & $\mathrm{w}$ & 315.4 & acerub & 21.7 & $\mathrm{w}$ \\
\hline & & 36.9 & & 317.6 & frapro & 23.7 & $\mathrm{w}$ \\
\hline 258.8 & gleaqu & 15.0 & W & 318.2 & cepocc & 10.3 & $\mathrm{w}$ \\
\hline 259.2 & nysaqu & 48.8 & $\mathrm{w}$ & 318.5 & frapro & 20.6 & w \\
\hline 260.9 & ulmame & 25.9 & $\mathrm{w}$ & 319.7 & nysaqu & 36.4 & $\mathrm{w}$ \\
\hline 261.0 & & \# 73 & & 322.6 & nysaqu & 37.8 & $\mathrm{w}$ \\
\hline & & 10.3 & & 325.4 & & \# 75 & \\
\hline 2654 & froson & 10.0 & & 326.6 & plaaqu & 16.3 & w \\
\hline 205.4 & Iracar & 5.5 & W & 329.2 & snag & 11.7 & $\mathrm{w}$ \\
\hline & & 8.4 & & 330.2 & taxdis & 35.8 & $\mathrm{w}$ \\
\hline & & 12.9 & & 331.6 & nysaqu & 31.3 & w \\
\hline & & 7.7 & & 333.9 & taxdis & 37.2 & w \\
\hline 2650 & fracar & 10.0 & $w$ & 335.4 & plaaqu & 10.8 & $\mathrm{w}$ \\
\hline 205.9 & Iracar & 8.7 & W & 336.2 & fracar & 10.1 & $\mathrm{w}$ \\
\hline & & 10.4 & & 3425 & & 32.0 & \\
\hline & & 8.0 & & 342.5 & plaaqu & 6.6 & W \\
\hline 268.3 & fracar & 11.9 & $\mathrm{w}$ & 345.5 & taxdis & 35.9 & $\mathrm{w}$ \\
\hline 271.1 & acerub & 11.8 & $\mathrm{w}$ & 346.8 & nysaqu & 48.7 & w \\
\hline 272.7 & nysaqu & 38.6 & $\mathrm{w}$ & 3481 & fracar & 12.4 & \\
\hline 276.6 & nysaqu & 38.5 & W & 348.1 & fracar & 9.2 & W \\
\hline 280.9 & acerub & 55.0 & w & 350.1 & taxdis & 37.4 & w \\
\hline 283.2 & taxdis & 21.8 & $\mathrm{w}$ & 351.3 & taxdis & 20.5 & $\mathrm{w}$ \\
\hline 284.2 & taxdis & 53.0 & $\mathrm{w}$ & 351.8 & taxdis & 13.8 & w \\
\hline 284.3 & plaaqu & 18.6 & $\mathrm{w}$ & 3526 & & 10.6 & \\
\hline 286.4 & taxdis & 16.8 & $\mathrm{w}$ & 352.0 & plaaqu & 5.0 & W \\
\hline 287.7 & acerub & 22.3 & w & 352.9 & gleaqu & 22.3 & w \\
\hline 291.5 & & \# 74 & & 356.4 & frapro & 49.2 & $\mathrm{w}$ \\
\hline
\end{tabular}


D MS: The sampling width of this transect is 10 meters ( 5 meters on each side of the transect line) from $5.3 \mathrm{~m}$ to $413 \mathrm{~m}$ and from $825.6 \mathrm{~m}$ to $902.2 \mathrm{~m}$. The sampling width is 5 meters on the west side of the transect line from 413 to 825.6 and from 902.2 to 1003.7 (Continued)

\begin{tabular}{|c|c|c|c|}
\hline $\begin{array}{l}\text { Distance from } \\
\text { beginning of } \\
\text { transect, in } \mathrm{m}\end{array}$ & $\begin{array}{c}\text { Tree } \\
\text { species }\end{array}$ & $\begin{array}{l}\text { Dbh, } \\
\text { in } \mathrm{cm}\end{array}$ & Side \\
\hline 358.7 & \multicolumn{3}{|c|}{ marker \# 76} \\
\hline 358.8 & plaaqu & 12.7 & $\mathrm{w}$ \\
\hline 360.9 & taxdis & 52.4 & $\mathrm{w}$ \\
\hline 367.9 & nysaqu & 45.3 & $\mathrm{w}$ \\
\hline 369.1 & frapro & 24.9 & $\mathrm{w}$ \\
\hline 369.3 & acerub & 14.7 & $\mathrm{w}$ \\
\hline 369.9 & nysaqu & 20.1 & $\mathrm{w}$ \\
\hline 373.1 & nysaqu & 53.6 & $\mathrm{w}$ \\
\hline 376.1 & nysaqu & 22.6 & $\mathrm{w}$ \\
\hline 377.2 & acerub & 49.4 & $\mathrm{w}$ \\
\hline 381.3 & nysaqu & 56.2 & $\mathrm{w}$ \\
\hline 384.9 & nysaqu & 29.8 & $\mathrm{w}$ \\
\hline 385.4 & nysaqu & 40.8 & $\mathrm{w}$ \\
\hline 385.9 & nysaqu & 21.1 & $\mathrm{w}$ \\
\hline 386.3 & nysaqu & 31.2 & $\mathrm{w}$ \\
\hline 388.1 & nysaqu & 13.3 & $\mathrm{w}$ \\
\hline \multirow{3}{*}{390.1} & \multirow{3}{*}{ fracar } & 14.6 & \multirow{3}{*}{$\mathrm{w}$} \\
\hline & & 9.8 & \\
\hline & & 13.3 & \\
\hline 390.2 & taxdis & 35.5 & w \\
\hline 391.7 & \multicolumn{3}{|c|}{ marker \# 77} \\
\hline 392.5 & gleaqu & 26.3 & $\mathrm{w}$ \\
\hline 394.4 & nysaqu & 21.3 & $\mathrm{w}$ \\
\hline 403.3 & plaaqu & 20.6 & $\mathrm{w}$ \\
\hline \multirow{2}{*}{404.3} & \multirow{2}{*}{ plaaqu } & 19.4 & \multirow{2}{*}{ w } \\
\hline & & 15.9 & \\
\hline 404.6 & plaaqu & 17.4 & $\mathrm{w}$ \\
\hline 404.6 & plaaqu & 5.1 & $\mathrm{w}$ \\
\hline 404.6 & plaaqu & 5.2 & $\mathrm{w}$ \\
\hline 406.7 & nysaqu & 57.0 & $\mathrm{w}$ \\
\hline \multirow{2}{*}{408.0} & \multirow{2}{*}{ plaaqu } & 12.3 & \multirow{2}{*}{$\mathrm{w}$} \\
\hline & & 16.4 & \\
\hline \multirow{3}{*}{415.6} & \multirow{3}{*}{ fracar } & 10.4 & \multirow{3}{*}{$\mathrm{w}$} \\
\hline & & 17.8 & \\
\hline & & 15.5 & \\
\hline 420.7 & \multicolumn{3}{|c|}{ marker \# 78} \\
\hline \multirow{2}{*}{420.8} & \multirow{2}{*}{ plaaqu } & 23.0 & \multirow{2}{*}{$\mathrm{w}$} \\
\hline & & 10.0 & \\
\hline 422.1 & plaaqu & 13.8 & $\mathrm{w}$ \\
\hline 425.1 & nysbif & 85.4 & $\mathrm{w}$ \\
\hline 425.9 & plaaqu & 26.5 & $\mathrm{w}$ \\
\hline 430.4 & plaaqu & 11.9 & $\mathrm{w}$ \\
\hline 431.6 & plaaqu & 13.1 & $\mathrm{w}$ \\
\hline \multirow{2}{*}{432.7} & \multirow{2}{*}{ nysaqu } & 37.9 & \multirow{2}{*}{ w } \\
\hline & & 38.3 & \\
\hline 437.0 & nysaqu & 29.0 & $\mathrm{w}$ \\
\hline 437.8 & taxdis & 27.2 & $\mathrm{w}$ \\
\hline
\end{tabular}

\begin{tabular}{|c|c|c|c|}
\hline $\begin{array}{l}\text { Distance from } \\
\text { beginning of } \\
\text { transect, in } \mathrm{m}\end{array}$ & $\begin{array}{c}\text { Tree } \\
\text { species }\end{array}$ & $\begin{array}{l}\text { Dbh, } \\
\text { in cm }\end{array}$ & Side \\
\hline 437.9 & acerub & 18.9 & $\mathrm{~W}$ \\
\hline 439.9 & nysaqu & 25.2 & $\mathrm{w}$ \\
\hline 444.0 & nysaqu & 33.3 & w \\
\hline 445.0 & frapro & 38.0 & w \\
\hline 445.4 & frapro & 33.3 & $\mathrm{w}$ \\
\hline 445.9 & taxdis & 11.7 & $\mathrm{w}$ \\
\hline 450.4 & nysaqu & 32.5 & $\mathrm{w}$ \\
\hline 452.1 & plaaqu & 18.4 & w \\
\hline 452.7 & \multicolumn{3}{|c|}{ marker \# 79} \\
\hline 452.9 & nysaqu & 27.8 & $\mathrm{~W}$ \\
\hline 453.1 & betnig & 21.3 & $\mathrm{w}$ \\
\hline 454.5 & plaaqu & 14.0 & w \\
\hline \multirow{4}{*}{459.7} & \multirow{4}{*}{ fracar } & 10.3 & \multirow{4}{*}{$\mathrm{w}$} \\
\hline & & 8.4 & \\
\hline & & 6.4 & \\
\hline & & 9.3 & \\
\hline \multirow{3}{*}{466.3} & \multirow{3}{*}{ fracar } & 11.0 & \multirow{3}{*}{$\mathrm{w}$} \\
\hline & & 9.0 & \\
\hline & & 4.3 & \\
\hline 468.2 & nysaqu & 38.7 & $\mathrm{~W}$ \\
\hline \multirow{2}{*}{473.7} & \multirow{2}{*}{ plaaqu } & 18.5 & \multirow{2}{*}{$\mathrm{w}$} \\
\hline & & 6.2 & \\
\hline 474.1 & fracar & 14.0 & $\mathrm{~W}$ \\
\hline 474.2 & gleaqu & 35.0 & w \\
\hline \multirow{4}{*}{474.9} & \multirow{4}{*}{ fracar } & 12.2 & \multirow{4}{*}{$\mathrm{w}$} \\
\hline & & 4.8 & \\
\hline & & 6.0 & \\
\hline & & 5.2 & \\
\hline 475.4 & \multicolumn{3}{|c|}{ marker \# 80} \\
\hline 478.0 & plaaqu & 16.6 & $\mathrm{w}$ \\
\hline 479.2 & plaaqu & 22.0 & $\mathrm{w}$ \\
\hline \multirow{2}{*}{484.5} & \multirow{2}{*}{ plaaqu } & 26.3 & \multirow{2}{*}{$\mathrm{w}$} \\
\hline & & 10.6 & \\
\hline \multirow{2}{*}{488.2} & \multirow{2}{*}{ fracar } & 12.7 & \multirow{2}{*}{$\mathrm{w}$} \\
\hline & & 7.4 & \\
\hline \multirow{4}{*}{492.5} & \multirow{4}{*}{ plaaqu } & 16.4 & \multirow{4}{*}{$\mathrm{w}$} \\
\hline & & 32.8 & \\
\hline & & 20.0 & \\
\hline & & 9.8 & \\
\hline & & 11.8 & \\
\hline 499.5 & fracar & 8.8 & $\mathrm{w}$ \\
\hline & & 7.2 & \\
\hline 500.8 & caraqu & 18.7 & $\mathrm{w}$ \\
\hline 502.4 & nysaqu & 61.0 & $\mathrm{w}$ \\
\hline 502.8 & plaaqu & 14.3 & $\mathrm{w}$ \\
\hline 504.5 & fracar & 11.8 & $\mathrm{w}$ \\
\hline 504.9 & fracar & 19.3 & $\mathrm{w}$ \\
\hline
\end{tabular}


D MS: The sampling width of this transect is 10 meters ( 5 meters on each side of the transect line) from $5.3 \mathrm{~m}$ to $413 \mathrm{~m}$ and from $825.6 \mathrm{~m}$ to $902.2 \mathrm{~m}$. The sampling width is 5 meters on the west side of the transect line from 413 to 825.6 and from 902.2 to 1003.7 (Continued)

\begin{tabular}{|c|c|c|c|c|c|c|c|}
\hline $\begin{array}{l}\text { Distance from } \\
\text { beginning of } \\
\text { transect, in } \mathrm{m}\end{array}$ & $\begin{array}{c}\text { Tree } \\
\text { species }\end{array}$ & $\begin{array}{l}\text { Dbh, } \\
\text { in cm }\end{array}$ & Side & $\begin{array}{l}\text { Distance from } \\
\text { beginning of } \\
\text { transect, in } \mathrm{m}\end{array}$ & $\begin{array}{c}\text { Tree } \\
\text { species }\end{array}$ & $\begin{array}{l}\text { Dbh, } \\
\text { in cm }\end{array}$ & Side \\
\hline 511.0 & nysaqu & 17.2 & $\mathrm{w}$ & 587.7 & \multicolumn{3}{|c|}{ marker \# 83} \\
\hline 513.3 & taxdis & 44.0 & w & 593.2 & betnig & 40.4 & w \\
\hline 514.6 & cepocc & 16.4 & $\mathrm{w}$ & 594.0 & betnig & 11.1 & $\mathrm{e}$ \\
\hline 515.4 & \multicolumn{3}{|c|}{ marker \# 81} & 595.0 & fracar & 10.2 & $\mathrm{w}$ \\
\hline \multirow{6}{*}{520.3} & \multirow{6}{*}{ fracar } & 12.5 & \multirow{6}{*}{$\mathrm{w}$} & 596.4 & betnig & 10.5 & $\mathrm{e}$ \\
\hline & & 5.0 & & 596.4 & foracu & 10.5 & $\mathrm{e}$ \\
\hline & & 10.3 & & \multirow{3}{*}{601.8} & \multirow{3}{*}{ fracar } & 16.7 & \multirow{3}{*}{$\mathrm{w}$} \\
\hline & & 5.7 & & & & 8.9 & \\
\hline & & 5.4 & & & & 4.1 & \\
\hline & & 11.8 & & \multirow{5}{*}{604.2} & \multirow{5}{*}{ fracar } & 10.0 & \multirow{5}{*}{$\mathrm{w}$} \\
\hline 521.2 & fracar & 18.1 & $\mathrm{w}$ & & & 8.9 & \\
\hline \multirow{5}{*}{523.5} & \multirow{5}{*}{ fracar } & 11.2 & \multirow{5}{*}{$\mathrm{w}$} & & & 8.7 & \\
\hline & & 6.1 & & & & 7.3 & \\
\hline & & 7.9 & & & & 9.0 & \\
\hline & & 5.5 & & \multirow{5}{*}{608.3} & \multirow{5}{*}{ fracar } & 10.0 & \multirow{5}{*}{$\mathrm{w}$} \\
\hline & & 6.2 & & & & 6.8 & \\
\hline 527.0 & nysaqu & 25.5 & $\mathrm{w}$ & & & 7.0 & \\
\hline \multirow{4}{*}{528.9} & \multirow{4}{*}{ fracar } & 16.3 & \multirow{4}{*}{$\mathrm{w}$} & & & 6.7 & \\
\hline & & 10.2 & & & & 7.0 & \\
\hline & & 6.4 & & 612.3 & crafla & 13.9 & $\mathrm{w}$ \\
\hline & & 9.2 & & 613.5 & diovir & 24.2 & $\mathrm{w}$ \\
\hline 529.7 & fracar & 15.5 & $\mathrm{~W}$ & 614.1 & fracar & 21.0 & w \\
\hline 537.1 & gleaqu & 22.5 & $\mathrm{w}$ & 617.5 & betnig & 13.4 & $\mathrm{w}$ \\
\hline 537.5 & cepocc & 11.0 & $\mathrm{w}$ & 619.7 & caraqu & 76.8 & $\mathrm{w}$ \\
\hline 538.1 & betnig & 34.4 & $\mathrm{w}$ & 621.1 & crafla & 22.3 & $\mathrm{w}$ \\
\hline 541.5 & betnig & 25.0 & $\mathrm{w}$ & \multirow{2}{*}{621.8} & fracor & 12.5 & e \\
\hline 545.9 & taxdis & 46.3 & $\mathrm{w}$ & & fracar & 8.3 & $\mathrm{e}$ \\
\hline 547.5 & plaaqu & 21.5 & w & 622.4 & fracar & 11.1 & $\mathrm{e}$ \\
\hline 552.0 & betnig & 19.3 & w & 624.0 & betnig & 30.6 & $\mathrm{e}$ \\
\hline 552.2 & betnig & 13.0 & $\mathrm{w}$ & 625.6 & betnig & 28.6 & w \\
\hline 553.1 & & $\# 82$ & & 625.6 & fracar & 10.1 & $\mathrm{~W}$ \\
\hline 554.0 & betnig & 15.5 & w & 627.4 & liqsty & 13.4 & $\mathrm{e}$ \\
\hline 557.9 & betnig & 22.1 & $\mathrm{w}$ & 628.5 & betnig & 17.5 & $\mathrm{e}$ \\
\hline 558.3 & betnig & 14.5 & $\mathrm{~W}$ & 630.9 & betnig & 29.3 & $\mathrm{e}$ \\
\hline 559.4 & taxdis & 29.9 & $\mathrm{w}$ & 631.2 & fracar & 10.5 & $\mathrm{~W}$ \\
\hline 559.5 & betnig & 20.2 & $\mathrm{w}$ & & & 13.3 & \\
\hline 563.2 & taxdis & 16.7 & $\mathrm{w}$ & & & 8.9 & \\
\hline 566.0 & ulmame & 31.0 & w & 6210 & feros & 5.7 & 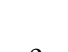 \\
\hline 569.7 & taxdis & 26.2 & $\mathrm{w}$ & 631.2 & fracar & 8.6 & e \\
\hline 571.4 & ulmame & 31.8 & w & & & 6.4 & \\
\hline 571.6 & betnig & 40.4 & w & & & 6.7 & \\
\hline 572.1 & plaaqu & 11.1 & W & (3) & c & 13.0 & 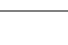 \\
\hline 574.6 & crafla & 14.7 & $\mathrm{w}$ & 632.0 & tracar & 12.1 & W \\
\hline 579.8 & taxdis & 31.6 & $\mathrm{w}$ & 634.8 & fracar & 14.3 & $\mathrm{w}$ \\
\hline 580.4 & betnig & 16.0 & $\mathrm{w}$ & 635.1 & gleaqu & 42.7 & $\mathrm{e}$ \\
\hline 584.9 & taxdis & 20.9 & w & 635.1 & taxdis & 27.1 & w \\
\hline 587.1 & fracar & 13.5 & $\mathrm{w}$ & 635.6 & betnig & 22.9 & $\mathrm{e}$ \\
\hline
\end{tabular}


D MS: The sampling width of this transect is 10 meters ( 5 meters on each side of the transect line) from $5.3 \mathrm{~m}$ to $413 \mathrm{~m}$ and from $825.6 \mathrm{~m}$ to $902.2 \mathrm{~m}$. The sampling width is 5 meters on the west side of the transect line from 413 to 825.6 and from 902.2 to 1003.7 (Continued)

\begin{tabular}{|c|c|c|c|}
\hline $\begin{array}{l}\text { Distance from } \\
\text { beginning of } \\
\text { transect, in } \mathrm{m}\end{array}$ & $\begin{array}{c}\text { Tree } \\
\text { species }\end{array}$ & $\begin{array}{l}\text { Dbh, } \\
\text { in cm }\end{array}$ & Side \\
\hline 639.2 & \multicolumn{3}{|c|}{ marker \# 84} \\
\hline 639.3 & betnig & 25.5 & $\mathrm{w}$ \\
\hline \multirow{2}{*}{639.9} & \multirow{2}{*}{ fracar } & 11.5 & \multirow{2}{*}{$\mathrm{w}$} \\
\hline & & 7.6 & \\
\hline 640.3 & fracar & 13.1 & $\mathrm{~W}$ \\
\hline 640.4 & betnig & 14.0 & w \\
\hline 640.6 & betnig & 20.7 & $\mathrm{e}$ \\
\hline 642.8 & plaaqu & 17.3 & w \\
\hline 644.3 & fracar & 10.5 & $\mathrm{w}$ \\
\hline 647.1 & taxdis & 78.3 & w \\
\hline 648.0 & fracar & 13.1 & $\mathrm{e}$ \\
\hline 649.7 & plaaqu & 11.5 & w \\
\hline 649.9 & plaaqu & 17.8 & $\mathrm{w}$ \\
\hline 650.6 & betnig & 29.0 & w \\
\hline 651.7 & nysaqu & 35.0 & $\mathrm{e}$ \\
\hline \multirow{2}{*}{654.1} & \multirow{2}{*}{ fracar } & 12.0 & \multirow{2}{*}{ w } \\
\hline & & 8.5 & \\
\hline 656.7 & fracar & 10.5 & $\mathrm{e}$ \\
\hline 656.7 & taxdis & 11.9 & w \\
\hline 657.0 & fracar & 10.1 & $\mathrm{e}$ \\
\hline 657.5 & fracar & 10.5 & $\mathrm{w}$ \\
\hline 658.0 & fracar & 10.8 & w \\
\hline 660.0 & nysaqu & 32.0 & $\mathrm{w}$ \\
\hline 661.8 & fracar & 15.2 & $\mathrm{w}$ \\
\hline 662.4 & quelau & 33.0 & $\mathrm{e}$ \\
\hline 662.7 & ulmame & 17.0 & $\mathrm{e}$ \\
\hline \multirow{2}{*}{665.4} & \multirow{2}{*}{ plaaqu } & 51.0 & \multirow{2}{*}{$\mathrm{e}$} \\
\hline & & 5.6 & \\
\hline 665.5 & taxdis & 32.0 & $\mathrm{w}$ \\
\hline 667.7 & fracar & 10.5 & $\mathrm{w}$ \\
\hline 668.9 & fracar & 13.4 & $\mathrm{w}$ \\
\hline \multirow{3}{*}{670.6} & \multirow{3}{*}{ fracar } & 15.0 & \multirow{3}{*}{ w } \\
\hline & & 7.1 & \\
\hline & & 8.7 & \\
\hline 670.7 & betnig & 12.2 & $\mathrm{~W}$ \\
\hline \multirow{2}{*}{671.1} & \multirow{2}{*}{ fracar } & 12.7 & \multirow{2}{*}{ w } \\
\hline & & 14.0 & \\
\hline 673.6 & cepocc & 7.6 & $\mathrm{~W}$ \\
\hline 674.6 & серосc & 10.0 & $\mathrm{w}$ \\
\hline \multirow{2}{*}{674.9} & \multirow{2}{*}{ plaaqu } & 36.0 & \multirow{2}{*}{ w } \\
\hline & & 11.1 & \\
\hline 675.4 & fracar & 10.2 & $\mathrm{e}$ \\
\hline 676.2 & fracar & 11.1 & $\mathrm{e}$ \\
\hline 676.2 & \multicolumn{3}{|c|}{ marker \# 85} \\
\hline 676.7 & betnig & 26.0 & $\mathrm{e}$ \\
\hline 676.8 & acerub & 11.6 & w \\
\hline 678.0 & cepocc & 11.1 & w \\
\hline
\end{tabular}

\begin{tabular}{|c|c|c|c|}
\hline $\begin{array}{l}\text { Distance from } \\
\text { beginning of } \\
\text { transect, in } \mathrm{m}\end{array}$ & $\begin{array}{c}\text { Tree } \\
\text { species }\end{array}$ & $\begin{array}{l}\text { Dbh, } \\
\text { in cm }\end{array}$ & Side \\
\hline 678.2 & fracar & 13.1 & $\mathrm{~W}$ \\
\hline 680.2 & fracar & 10.0 & $\mathrm{e}$ \\
\hline \multirow{2}{*}{680.3} & \multirow{2}{*}{ plaaqu } & 13.2 & \multirow{2}{*}{$\mathrm{w}$} \\
\hline & & 4.9 & \\
\hline \multirow{2}{*}{680.5} & \multirow{2}{*}{ fracar } & 10.6 & \multirow{2}{*}{ e } \\
\hline & & 7.9 & \\
\hline \multirow{2}{*}{680.6} & \multirow{2}{*}{ plaaqu } & 35.5 & \multirow{2}{*}{$\mathrm{w}$} \\
\hline & & 16.4 & \\
\hline \multirow{2}{*}{681.7} & \multirow{2}{*}{ plaaqu } & 16.4 & \multirow{2}{*}{$\mathrm{w}$} \\
\hline & & 15.0 & \\
\hline 685.6 & plaaqu & 11.5 & $\mathrm{e}$ \\
\hline \multirow{2}{*}{686.0} & \multirow{2}{*}{ plaaqu } & 35.5 & \multirow{2}{*}{ e } \\
\hline & & 30.6 & \\
\hline 686.0 & taxdis & 19.4 & $\mathrm{w}$ \\
\hline 688.6 & fracar & 10.2 & $\mathrm{w}$ \\
\hline 689.6 & fracar & 13.1 & $\mathrm{w}$ \\
\hline \multirow{2}{*}{689.9} & \multirow{2}{*}{ fracar } & 10.0 & \multirow{2}{*}{ e } \\
\hline & & 8.0 & \\
\hline 691.2 & gleaqu & 48.0 & $\mathrm{w}$ \\
\hline 692.0 & nysaqu & 35.7 & $\mathrm{w}$ \\
\hline 692.3 & fracar & 10.0 & $\mathrm{e}$ \\
\hline 693.2 & nysaqu & 42.0 & $\mathrm{e}$ \\
\hline 693.3 & fracar & 10.2 & $\mathrm{w}$ \\
\hline \multirow{2}{*}{693.4} & \multirow{2}{*}{ betnig } & 19.2 & \multirow{2}{*}{$\mathrm{W}$} \\
\hline & & 24.3 & \\
\hline 693.8 & plaaqu & 10.8 & $\mathrm{w}$ \\
\hline 694.2 & plaaqu & 15.3 & $\mathrm{e}$ \\
\hline 695.2 & nysaqu & 18.6 & $\mathrm{w}$ \\
\hline 697.6 & fracar & 12.4 & $\mathrm{e}$ \\
\hline 698.7 & nysaqu & 35.0 & $\mathrm{w}$ \\
\hline 699.1 & plaaqu & 11.0 & $\mathrm{w}$ \\
\hline 699.4 & plaaqu & 16.0 & $\mathrm{w}$ \\
\hline 699.5 & fracar & 13.2 & $\mathrm{e}$ \\
\hline 699.7 & fracar & 11.1 & $\mathrm{e}$ \\
\hline \multirow{3}{*}{699.7} & fracar & \multirow{3}{*}{10.0} & \\
\hline & fracar & & $\mathrm{e}$ \\
\hline & fracar & & \\
\hline 701.5 & plaaqu & 19.6 & $\mathrm{e}$ \\
\hline 702.9 & acerub & 18.4 & $\mathrm{w}$ \\
\hline 703.2 & gleaqu & 26.7 & $\mathrm{e}$ \\
\hline 703.2 & plaaqu & 17.2 & $\mathrm{e}$ \\
\hline 7037 & plaaqu & 31.4 & w \\
\hline & & 21.2 & $\mathrm{w}$ \\
\hline 7038 & nlaagu & 16.7 & w \\
\hline 100.0 & pradqu & 10.6 & $\mathrm{~W}$ \\
\hline 703.9 & gleaqu & 18.0 & e \\
\hline 704.0 & & \# 86 & \\
\hline
\end{tabular}


D MS: The sampling width of this transect is 10 meters ( 5 meters on each side of the transect line) from $5.3 \mathrm{~m}$ to $413 \mathrm{~m}$ and from $825.6 \mathrm{~m}$ to $902.2 \mathrm{~m}$. The sampling width is 5 meters on the west side of the transect line from 413 to 825.6 and from 902.2 to 1003.7 (Continued)

\begin{tabular}{|c|c|c|c|c|c|c|c|}
\hline $\begin{array}{l}\text { Distance from } \\
\text { beginning of } \\
\text { transect, in } \mathrm{m}\end{array}$ & $\begin{array}{c}\text { Tree } \\
\text { species }\end{array}$ & $\begin{array}{l}\text { Dbh, } \\
\text { in cm }\end{array}$ & Side & $\begin{array}{l}\text { Distance from } \\
\text { beginning of } \\
\text { transect, in } \mathrm{m}\end{array}$ & $\begin{array}{c}\text { Tree } \\
\text { species }\end{array}$ & $\begin{array}{l}\text { Dbh, } \\
\text { in cm }\end{array}$ & Side \\
\hline \multirow{2}{*}{706.5} & \multirow{2}{*}{ nysaqu } & 30.0 & \multirow{2}{*}{$\mathrm{e}$} & 725.7 & taxdis & 31.9 & $\mathrm{w}$ \\
\hline & & 13.2 & & 726.5 & fracar & 11.8 & $\mathrm{w}$ \\
\hline 707.5 & betnig & 18.5 & $\mathrm{e}$ & 726.8 & taxdis & 49.9 & $\mathrm{w}$ \\
\hline \multirow{2}{*}{707.5} & \multirow{2}{*}{ fracar } & 6.3 & \multirow{2}{*}{$\mathrm{w}$} & 728.0 & nysaqu & 15.7 & $\mathrm{e}$ \\
\hline & & 10.1 & & 728.1 & fracar & 14.6 & $\mathrm{w}$ \\
\hline 707.8 & plaaqu & 17.6 & $\mathrm{e}$ & \multirow{2}{*}{728.4} & \multirow{2}{*}{ plaaqu } & 23.5 & \multirow{2}{*}{ e } \\
\hline 708.5 & nysaqu & 23.5 & $\mathrm{w}$ & & & 8.3 & \\
\hline 709.6 & cepocc & 10.2 & $\mathrm{w}$ & \multirow{2}{*}{729.2} & \multirow{2}{*}{ taxdis } & 31.0 & \multirow{2}{*}{$\mathrm{e}$} \\
\hline \multirow{3}{*}{709.6} & \multirow{3}{*}{ fracar } & 10.5 & \multirow{3}{*}{$\mathrm{w}$} & & & 14.0 & \\
\hline & & 7.6 & & \multirow{4}{*}{729.8} & \multirow{4}{*}{ plaaqu } & 6.6 & \multirow{4}{*}{$\mathrm{w}$} \\
\hline & & 5.7 & & & & 13.7 & \\
\hline 710.0 & plaaqu & 12.0 & $\mathrm{e}$ & & & 17.3 & \\
\hline 710.2 & nysaqu & 31.1 & $\mathrm{~W}$ & & & 11.2 & \\
\hline 710.6 & plaaqu & 11.1 & $\mathrm{e}$ & \multirow{2}{*}{729.9} & \multirow{2}{*}{ fracar } & 14.0 & \multirow{2}{*}{ e } \\
\hline 710.7 & plaaqu & 10.3 & $\mathrm{e}$ & & & 9.1 & \\
\hline 711.7 & betnig & 21.3 & $\mathrm{e}$ & \multirow{2}{*}{730.0} & fracar & 14.3 & e \\
\hline & & 8.7 & & & fracar & 9.1 & e \\
\hline 712.2 & fracar & 7.9 & $\mathrm{w}$ & 732.5 & nysaqu & 35.2 & $\mathrm{~W}$ \\
\hline & & 8.0 & & 735.8 & gleaqu & 12.2 & $\mathrm{e}$ \\
\hline & & 11.4 & & & & 22.0 & \\
\hline 712.5 & fracar & 9.3 & $\mathrm{~W}$ & 736.6 & taxdis & 42.0 & $\mathrm{e}$ \\
\hline & & 10.5 & & 737.8 & & \# 87 & \\
\hline 7125 & frocor & 10.5 & $w$ & 7384 & nусаян & 32.0 & e \\
\hline 712.5 & tracar & 5.1 & W & 138.4 & nysaqu & 32.2 & e \\
\hline & & 8.9 & & 738.6 & taxdis & 20.0 & $\mathrm{e}$ \\
\hline 712.8 & salcar & 37.1 & $\mathrm{e}$ & 738.8 & taxdis & 39.4 & $\mathrm{e}$ \\
\hline & & 10.8 & & 739.9 & taxdis & 38.0 & $\mathrm{w}$ \\
\hline 716.4 & betnig & 7.1 & $\mathrm{e}$ & 740.2 & quelau & 43.4 & $\mathrm{e}$ \\
\hline & & 6.3 & & 740.4 & taxdis & 11.3 & $\mathrm{e}$ \\
\hline 716.7 & nysaqu & 23.5 & W & 740.5 & taxdis & 14.9 & $\mathrm{e}$ \\
\hline 716.8 & nysaqu & 31.4 & $\mathrm{w}$ & 740.7 & plaaqu & 17.5 & $\mathrm{w}$ \\
\hline 717.3 & betnig & 12.0 & $\mathrm{e}$ & 741.0 & taxdis & 14.7 & $\mathrm{e}$ \\
\hline 717.5 & betnig & 13.3 & $\mathrm{e}$ & 741.1 & taxdis & 13.0 & $\mathrm{e}$ \\
\hline 718.3 & nysaqu & 61.0 & $\mathrm{e}$ & 741.4 & taxdis & 14.3 & $\mathrm{e}$ \\
\hline 7104 & ( & 48.0 & 3 & 741.5 & plaaqu & 14.3 & $\mathrm{~W}$ \\
\hline 118.4 & acerub & 6.4 & W & 741.5 & taxdis & 16.1 & $\mathrm{e}$ \\
\hline 720.2 & ulmame & 13.2 & W & 741.9 & taxdis & 27.8 & $\mathrm{e}$ \\
\hline 722.4 & betnig & 15.5 & W & 742.1 & cepocc & 14.6 & $\mathrm{w}$ \\
\hline 722.8 & taxdis & 35.5 & $\mathrm{w}$ & 742.2 & plaaqu & 17.0 & $\mathrm{w}$ \\
\hline 724.6 & taxdis & 47.2 & $\mathrm{w}$ & 742.6 & taxdis & 14.7 & $\mathrm{e}$ \\
\hline 724.7 & betnig & 13.6 & $\mathrm{e}$ & 742.7 & fracar & 10.7 & $\mathrm{e}$ \\
\hline 724.8 & taxdis & 31.2 & W & 742.9 & taxdis & 20.1 & $\mathrm{e}$ \\
\hline 725.2 & corfoe & 11.9 & $\mathrm{e}$ & 743.4 & taxdis & 32.5 & $\mathrm{e}$ \\
\hline 725.2 & taxdis & 20.0 & w & 743.4 & taxdis & 17.6 & $\mathrm{e}$ \\
\hline 725.5 & taxdis & 14.4 & $\mathrm{w}$ & 743.7 & taxdis & 10.5 & $\mathrm{e}$ \\
\hline 725.6 & nysaqu & 42.0 & $\mathrm{e}$ & 744.2 & taxdis & 19.3 & $\mathrm{e}$ \\
\hline
\end{tabular}


D MS: The sampling width of this transect is 10 meters ( 5 meters on each side of the transect line) from $5.3 \mathrm{~m}$ to $413 \mathrm{~m}$ and from $825.6 \mathrm{~m}$ to $902.2 \mathrm{~m}$. The sampling width is 5 meters on the west side of the transect line from 413 to 825.6 and from 902.2 to 1003.7 (Continued)

\begin{tabular}{|c|c|c|c|}
\hline $\begin{array}{l}\text { Distance from } \\
\text { beginning of } \\
\text { transect, in } \mathrm{m}\end{array}$ & $\begin{array}{c}\text { Tree } \\
\text { species }\end{array}$ & $\begin{array}{l}\text { Dbh, } \\
\text { in cm }\end{array}$ & Side \\
\hline 744.3 & taxdis & 22.0 & $\mathrm{e}$ \\
\hline \multirow{4}{*}{746.5} & \multirow{4}{*}{ fracar } & 10.3 & \multirow{4}{*}{$\mathrm{e}$} \\
\hline & & 8.1 & \\
\hline & & 5.1 & \\
\hline & & 8.0 & \\
\hline 747.2 & fracar & 13.2 & $\mathrm{w}$ \\
\hline \multirow{2}{*}{749.1} & \multirow{2}{*}{ taxdis } & 30.6 & \multirow{2}{*}{$\mathrm{e}$} \\
\hline & & 24.7 & \\
\hline 749.2 & taxdis & 31.2 & w \\
\hline 749.6 & taxdis & 55.0 & $\mathrm{w}$ \\
\hline \multirow{2}{*}{749.7} & \multirow{2}{*}{ plaaqu } & 18.8 & \multirow{2}{*}{$\mathrm{e}$} \\
\hline & & 24.0 & \\
\hline 750.0 & fracar & 14.4 & $\mathrm{e}$ \\
\hline 754.7 & cepocc & 18.0 & w \\
\hline \multirow{3}{*}{755.8} & \multirow{3}{*}{ fracar } & 13.3 & \multirow{3}{*}{$\mathrm{e}$} \\
\hline & & 6.3 & \\
\hline & & 4.7 & \\
\hline \multirow{4}{*}{756.9} & \multirow{4}{*}{ fracar } & 12.6 & \multirow{4}{*}{$\mathrm{e}$} \\
\hline & & 13.9 & \\
\hline & & 12.0 & \\
\hline & & 13.5 & \\
\hline 757.7 & gleaqu & 31.0 & $\mathrm{~W}$ \\
\hline 760.4 & fracar & 13.1 & $\mathrm{w}$ \\
\hline 760.4 & fracar & 14.7 & w \\
\hline \multirow{2}{*}{760.7} & \multirow{2}{*}{ fracar } & 7.8 & \multirow{2}{*}{$\mathrm{w}$} \\
\hline & & 14.9 & \\
\hline \multirow{3}{*}{760.8} & \multirow{3}{*}{ fracar } & 15.5 & \multirow{3}{*}{$\mathrm{w}$} \\
\hline & & 8.1 & \\
\hline & & 12.2 & \\
\hline 762.0 & plaaqu & 18.2 & $\mathrm{e}$ \\
\hline 762.1 & plaaqu & 24.7 & $\mathrm{e}$ \\
\hline 763.0 & plaaqu & 18.6 & $\mathrm{e}$ \\
\hline 763.6 & plaaqu & 16.1 & $\mathrm{e}$ \\
\hline 766.0 & betnig & 16.2 & $\mathrm{e}$ \\
\hline 767.7 & taxdis & 11.0 & $\mathrm{w}$ \\
\hline 768.2 & plaaqu & 15.7 & w \\
\hline \multirow{5}{*}{769.5} & \multirow{5}{*}{ plaaqu } & 12.3 & \multirow{5}{*}{$\mathrm{e}$} \\
\hline & & 12.5 & \\
\hline & & 15.0 & \\
\hline & & 24.5 & \\
\hline & & 57.0 & \\
\hline 771.4 & gleaqu & 44.0 & $\mathrm{~W}$ \\
\hline 772.1 & fracar & 11.5 & $\mathrm{w}$ \\
\hline 774.0 & & $\# 88$ & \\
\hline 775.6 & betnig & 17.6 & $\mathrm{w}$ \\
\hline
\end{tabular}

\begin{tabular}{|c|c|c|c|}
\hline $\begin{array}{l}\text { Distance from } \\
\text { beginning of } \\
\text { transect, in } \mathrm{m}\end{array}$ & $\begin{array}{c}\text { Tree } \\
\text { species }\end{array}$ & $\begin{array}{l}\text { Dbh, } \\
\text { in } \mathrm{cm}\end{array}$ & Side \\
\hline 776.0 & plaaqu & 22.5 & $\mathrm{e}$ \\
\hline \multirow{2}{*}{776.0} & \multirow{2}{*}{ plaaqu } & 5.6 & \multirow{2}{*}{$\mathrm{e}$} \\
\hline & & 10.1 & \\
\hline \multirow{3}{*}{776.7} & \multirow{3}{*}{ plaaqu } & 12.4 & \multirow{3}{*}{$\mathrm{e}$} \\
\hline & & 10.8 & \\
\hline & & 12.2 & \\
\hline \multirow{4}{*}{777.1} & \multirow{4}{*}{ fracar } & 14.7 & \multirow{4}{*}{$\mathrm{e}$} \\
\hline & & 5.0 & \\
\hline & & 4.7 & \\
\hline & & 5.4 & \\
\hline 779.2 & betnig & 13.5 & $\mathrm{w}$ \\
\hline 779.6 & betnig & 28.2 & $\mathrm{e}$ \\
\hline 782.7 & taxdis & 16.0 & $\mathrm{w}$ \\
\hline 783.1 & taxdis & 27.2 & $\mathrm{e}$ \\
\hline 785.0 & taxdis & 22.8 & $\mathrm{e}$ \\
\hline 788.2 & cravir & 21.2 & $\mathrm{e}$ \\
\hline 788.4 & snag & 19.5 & $\mathrm{e}$ \\
\hline 788.9 & acerub & 21.9 & $\mathrm{e}$ \\
\hline 791.0 & acerub & 54.3 & $\mathrm{e}$ \\
\hline 791.6 & taxdis & 15.3 & w \\
\hline 792.6 & ulmame & 37.8 & $\mathrm{e}$ \\
\hline 792.9 & quelyr & 20.3 & $\mathrm{e}$ \\
\hline 796.6 & taxdis & 17.2 & $\mathrm{w}$ \\
\hline 798.2 & taxdis & 19.8 & $\mathrm{e}$ \\
\hline 800.3 & taxdis & 15.4 & w \\
\hline 801.6 & betnig & 44.4 & $\mathrm{e}$ \\
\hline 801.8 & caraqu & 29.0 & w \\
\hline 802.1 & quelyr & 24.6 & w \\
\hline 802.7 & caraqu & 10.5 & w \\
\hline 803.3 & snag & 10.9 & $\mathrm{e}$ \\
\hline 804.0 & taxdis & 12.7 & $\mathrm{e}$ \\
\hline 804.3 & \multicolumn{3}{|c|}{ marker \# 89} \\
\hline 805.2 & taxdis & 16.3 & $\mathrm{w}$ \\
\hline 805.9 & betnig & 51.2 & $\mathrm{e}$ \\
\hline 806.4 & taxdis & 15.6 & $\mathrm{e}$ \\
\hline 806.8 & snag & 50.0 & $\mathrm{e}$ \\
\hline 807.9 & caraqu & 22.8 & w \\
\hline \multirow{2}{*}{810.6} & \multirow{2}{*}{ foracu } & 12.0 & \multirow{2}{*}{$\mathrm{e}$} \\
\hline & & 8.1 & \\
\hline 811.5 & betnig & 12.9 & $\mathrm{w}$ \\
\hline 813.4 & betnig & 17.7 & $\mathrm{e}$ \\
\hline 815.5 & acerub & 31.9 & w \\
\hline 816.8 & taxdis & 26.4 & $\mathrm{e}$ \\
\hline 817.9 & betnig & 21.8 & w \\
\hline 824.6 & caraqu & 45.0 & $\mathrm{e}$ \\
\hline
\end{tabular}


D MS: The sampling width of this transect is 10 meters ( 5 meters on each side of the transect line) from $5.3 \mathrm{~m}$ to $413 \mathrm{~m}$ and from $825.6 \mathrm{~m}$ to $902.2 \mathrm{~m}$. The sampling width is 5 meters on the west side of the transect line from 413 to 825.6 and from 902.2 to 1003.7 (Continued)

\begin{tabular}{|c|c|c|c|c|c|c|c|}
\hline $\begin{array}{l}\text { Distance from } \\
\text { beginning of } \\
\text { transect, in } \mathrm{m}\end{array}$ & $\begin{array}{c}\text { Tree } \\
\text { species }\end{array}$ & $\begin{array}{l}\text { Dbh, } \\
\text { in cm }\end{array}$ & Side & $\begin{array}{c}\text { Distance from } \\
\text { beginning of } \\
\text { transect, in } \mathrm{m}\end{array}$ & $\begin{array}{c}\text { Tree } \\
\text { species }\end{array}$ & $\begin{array}{l}\text { Dbh, } \\
\text { in cm }\end{array}$ & Side \\
\hline 826.3 & betnig & 18.7 & $\mathrm{w}$ & 924.2 & betnig & 50.8 & $\mathrm{w}$ \\
\hline \multirow{2}{*}{828.0} & \multirow{2}{*}{ foracu } & 9.7 & \multirow{2}{*}{ w } & 928.7 & taxdis & 15.7 & $\mathrm{e}$ \\
\hline & & 11.9 & & \multirow{3}{*}{929.8} & \multirow{3}{*}{ fracar } & 11.1 & \multirow{3}{*}{$\mathrm{w}$} \\
\hline 830.6 & ulmame & 15.0 & w & & & 11.0 & \\
\hline 831.4 & ulmame & 11.9 & $\mathrm{w}$ & & & 9.5 & \\
\hline 839.5 & caraqu & 43.1 & $\mathrm{w}$ & \multirow{2}{*}{930.1} & \multirow{2}{*}{ fracar } & 11.0 & \multirow{2}{*}{$\mathrm{w}$} \\
\hline 842.2 & acerub & 13.5 & w & & & 16.6 & \\
\hline 845.0 & \multicolumn{3}{|c|}{ marker \# 90} & 930.1 & fracar & 5.5 & $\mathrm{w}$ \\
\hline \multirow{2}{*}{845.8} & \multirow{2}{*}{ cravir } & 11.2 & \multirow{2}{*}{$\mathrm{w}$} & \multirow{4}{*}{930.6} & \multirow{4}{*}{ fracar } & 12.4 & \multirow{4}{*}{$\mathrm{w}$} \\
\hline & & 11.3 & & & & 10.5 & \\
\hline 845.8 & cravir & 6.9 & $\mathrm{w}$ & & & 11.2 & \\
\hline 846.3 & caraqu & 38.4 & $\mathrm{w}$ & & & 13.2 & \\
\hline 850.5 & quelyr & 46.4 & $\mathrm{e}$ & 931.8 & liqsty & 10.0 & $\mathrm{e}$ \\
\hline 854.9 & cravir & 16.8 & $\mathrm{e}$ & 935.0 & plaaqu & 30.0 & $\mathrm{e}$ \\
\hline 862.1 & taxdis & 10.2 & $\mathrm{e}$ & 936.1 & taxdis & 32.7 & $\mathrm{w}$ \\
\hline 863.9 & caraqu & 33.7 & $\mathrm{w}$ & 937.5 & plaaqu & 24.1 & $\mathrm{e}$ \\
\hline 864.1 & cellae & 22.7 & w & 938.3 & plaaqu & 25.4 & $\mathrm{w}$ \\
\hline 866.7 & betnig & 37.5 & $\mathrm{e}$ & 938.4 & cepocc & 10.7 & $\mathrm{e}$ \\
\hline 867.7 & caraqu & 50.0 & $\mathrm{e}$ & \multirow{8}{*}{942.7} & \multirow{8}{*}{ fracar } & 10.3 & \multirow{8}{*}{ e } \\
\hline 870.3 & caraqu & 34.4 & w & & & 9.3 & \\
\hline 873.1 & betnig & 10.7 & $\mathrm{e}$ & & & 5.0 & \\
\hline 873.7 & betnig & 16.2 & $\mathrm{w}$ & & & 8.0 & \\
\hline \multirow{2}{*}{877.0} & \multirow{2}{*}{ carcar } & 10.2 & 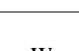 & & & 7.4 & \\
\hline & & 14.0 & W & & & 13.8 & \\
\hline 883.7 & betnig & 15.7 & w & & & 7.4 & \\
\hline 883.8 & betnig & 17.2 & $\mathrm{e}$ & & & 9.2 & \\
\hline 886.5 & betnig & 15.8 & $\mathrm{w}$ & 946.1 & fracar & 17.4 & $\mathrm{e}$ \\
\hline 889.3 & betnig & 22.9 & $\mathrm{e}$ & 946.3 & snag & 11.4 & $\mathrm{e}$ \\
\hline 889.5 & & \# 91 & & 946.4 & gleaqu & 38.4 & $\mathrm{e}$ \\
\hline 889.8 & liqsty & 24.1 & $\mathrm{e}$ & 947.0 & gleaqu & 13.0 & $\mathrm{e}$ \\
\hline 890.1 & betnig & 13.0 & w & 948.0 & gleaqu & 15.0 & $\mathrm{e}$ \\
\hline 891.5 & betnig & 18.9 & w & & & 27.9 & \\
\hline 891.5 & betnig & 15.6 & $\mathrm{e}$ & 950.6 & plaaqu & 35.6 & w \\
\hline 893.5 & betnig & 10.7 & $\mathrm{e}$ & 954.6 & nysaqu & 21.5 & $\mathrm{e}$ \\
\hline 894.5 & betnig & 23.7 & w & 955.4 & plaaqu & 37.5 & $\mathrm{w}$ \\
\hline 894.9 & betnig & 18.3 & w & 956.4 & gleaqu & 15.1 & $\mathrm{e}$ \\
\hline 896.3 & betnig & 10.0 & $\mathrm{w}$ & 956.4 & taxdis & 15.6 & $\mathrm{e}$ \\
\hline 897.7 & betnig & 11.9 & w & 956.8 & taxdis & 49.0 & $\mathrm{w}$ \\
\hline 897.7 & betnig & 10.3 & $\mathrm{e}$ & 959.0 & taxdis & 53.8 & w \\
\hline 899.0 & caraqu & 34.0 & $\mathrm{e}$ & 0500 & & 12.7 & 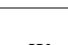 \\
\hline 900.4 & betnig & 12.9 & $\mathrm{e}$ & 959.9 & fracar & 6.5 & W \\
\hline 900.8 & betnig & 31.8 & $\mathrm{w}$ & 959.9 & taxdis & 35.0 & $\mathrm{w}$ \\
\hline 902.2 & betnig & 12.4 & $\mathrm{e}$ & 960.1 & plaaqu & 17.3 & w \\
\hline 903.4 & betnig & 15.7 & w & 960.5 & taxdis & 55.5 & $\mathrm{e}$ \\
\hline 916.4 & snag & 30.6 & $\mathrm{e}$ & 964.9 & plaaqu & 16.0 & $\mathrm{w}$ \\
\hline 917.4 & & \# 92 & & 965.4 & plaaqu & 21.0 & w \\
\hline 918.6 & snag & 69.0 & $\mathrm{e}$ & 966.4 & nysaqu & 63.0 & $\mathrm{e}$ \\
\hline
\end{tabular}


D MS: The sampling width of this transect is 10 meters (5 meters on each side of the transect line) from $5.3 \mathrm{~m}$ to $413 \mathrm{~m}$ and from $825.6 \mathrm{~m}$ to $902.2 \mathrm{~m}$. The sampling width is 5 meters on the west side of the transect line from 413 to 825.6 and from 902.2 to 1003.7 (Continued)

\begin{tabular}{|c|c|c|c|}
\hline $\begin{array}{l}\text { Distance from } \\
\text { beginning of } \\
\text { transect, in } m\end{array}$ & $\begin{array}{c}\text { Tree } \\
\text { species }\end{array}$ & $\begin{array}{l}\text { Dbh, } \\
\text { in cm }\end{array}$ & Side \\
\hline 967.9 & nysaqu & 45.9 & $\mathrm{w}$ \\
\hline 969.5 & nysaqu & 28.2 & $\mathrm{e}$ \\
\hline 969.9 & gleaqu & 34.8 & $\mathrm{~W}$ \\
\hline 970.0 & taxdis & 53.6 & $\mathrm{w}$ \\
\hline 970.1 & \multicolumn{3}{|c|}{ marker \# 93} \\
\hline 972.0 & taxdis & 63.8 & $\mathrm{w}$ \\
\hline 972.2 & snag & 37.0 & $\mathrm{e}$ \\
\hline 976.1 & quelyr & 61.1 & $\mathrm{e}$ \\
\hline 980.6 & caraqu & 61.2 & $\mathrm{~W}$ \\
\hline 980.8 & quelyr & 67.0 & $\mathrm{e}$ \\
\hline 984.0 & quelyr & 44.5 & $\mathrm{~W}$ \\
\hline 985.0 & ulmame & 26.4 & w \\
\hline 994.0 & snag & 53.9 & $\mathrm{w}$ \\
\hline 1000.2 & ulmame & 20.2 & $\mathrm{~W}$ \\
\hline 1002.9 & ulmame & 13.4 & W \\
\hline 1003.7 & \multicolumn{3}{|c|}{ marker \# 94} \\
\hline 1004.9 & snag & 36.1 & $\mathrm{w}$ \\
\hline 1005.3 & quelau & 60.0 & $\mathrm{e}$ \\
\hline 1007.5 & ulmame & 26.0 & $\mathrm{e}$ \\
\hline
\end{tabular}


E KN: The sampling width of this transect is 5 meters (on the north side of the transect line) from -3.1 to 721.5 and 10 meters ( 5 meters on either side of the transect line) from 721.5 to 729.3

\begin{tabular}{|c|c|c|c|}
\hline $\begin{array}{l}\text { Distance from } \\
\text { beginning of } \\
\text { transect, in } \mathrm{m}\end{array}$ & $\begin{array}{c}\text { Tree } \\
\text { species }\end{array}$ & $\begin{array}{l}\text { Dbh, } \\
\text { in cm }\end{array}$ & Side \\
\hline 0 & \multicolumn{3}{|c|}{ marker \# 95} \\
\hline 3.9 & liqsty & 10.0 & $\mathrm{n}$ \\
\hline 4.2 & betnig & 18.9 & $\mathrm{n}$ \\
\hline \multirow{2}{*}{6.2} & \multirow{2}{*}{ liqsty } & 10.5 & \multirow{2}{*}{$\mathrm{n}$} \\
\hline & & 6.5 & \\
\hline 7.9 & taxdis & 33.0 & $\mathrm{n}$ \\
\hline 10.8 & betnig & 12.6 & $\mathrm{n}$ \\
\hline 11.5 & betnig & 13.0 & $\mathrm{n}$ \\
\hline 12.4 & betnig & 10.0 & $\mathrm{n}$ \\
\hline 14.6 & betnig & 10.0 & $\mathrm{n}$ \\
\hline 17.4 & taxdis & 37.3 & $\mathrm{n}$ \\
\hline 18.5 & taxdis & 17.4 & $\mathrm{n}$ \\
\hline \multirow{2}{*}{22.4} & \multirow{2}{*}{ betnig } & 15.7 & \multirow{2}{*}{$\mathrm{n}$} \\
\hline & & 7.0 & \\
\hline 25.1 & taxdis & 11.4 & $\mathrm{n}$ \\
\hline 25.2 & \multicolumn{3}{|c|}{ marker \# 96} \\
\hline 27.7 & betnig & 12.3 & $\mathrm{n}$ \\
\hline \multirow{2}{*}{28.7} & \multirow{2}{*}{ betnig } & 8.9 & \multirow{2}{*}{$\mathrm{n}$} \\
\hline & & 11.2 & \\
\hline 30.4 & liqsty & 10.2 & $\mathrm{n}$ \\
\hline 32.3 & betnig & 18.8 & $\mathrm{n}$ \\
\hline 34.1 & taxdis & 35.4 & $\mathrm{n}$ \\
\hline 35.5 & taxdis & 35.4 & $\mathrm{n}$ \\
\hline 37.3 & liqsty & 12.3 & $\mathrm{n}$ \\
\hline 39.2 & betnig & 20.0 & $\mathrm{n}$ \\
\hline \multirow{3}{*}{41.2} & \multirow{3}{*}{ gleaqu } & 10.0 & \multirow{3}{*}{$\mathrm{n}$} \\
\hline & & 6.5 & \\
\hline & & 5.0 & \\
\hline 45.6 & plaaqu & 22.4 & $\mathrm{n}$ \\
\hline 50.7 & taxdis & 13.4 & $\mathrm{n}$ \\
\hline 51.6 & \multicolumn{3}{|c|}{ marker \# 97} \\
\hline \multirow{2}{*}{52.5} & \multirow{2}{*}{ salcar } & 13.3 & \multirow{2}{*}{$\mathrm{n}$} \\
\hline & & 8.7 & \\
\hline 52.6 & salcar & 15.4 & $\mathrm{n}$ \\
\hline 52.6 & salcar & 11.0 & $\mathrm{n}$ \\
\hline 54.0 & fracar & 12.5 & $\mathrm{n}$ \\
\hline 55.9 & plaaqu & 16.5 & $\mathrm{n}$ \\
\hline 59.8 & taxdis & 32.6 & $\mathrm{n}$ \\
\hline 60.8 & taxdis & 32.7 & $\mathrm{n}$ \\
\hline \multirow{2}{*}{61.0} & \multirow{2}{*}{ fracar } & 10.5 & \multirow{2}{*}{$\mathrm{n}$} \\
\hline & & 4.3 & \\
\hline 61.2 & nysaqu & 26.5 & $\mathrm{n}$ \\
\hline \multirow{3}{*}{61.5} & \multirow{3}{*}{ fracar } & 14.5 & \multirow{3}{*}{$\mathrm{n}$} \\
\hline & & 5.0 & \\
\hline & & 6.0 & \\
\hline
\end{tabular}

\begin{tabular}{|c|c|c|c|}
\hline $\begin{array}{l}\text { Distance from } \\
\text { beginning of } \\
\text { transect, in } \mathrm{m}\end{array}$ & $\begin{array}{c}\text { Tree } \\
\text { species }\end{array}$ & $\begin{array}{l}\text { Dbh, } \\
\text { in } \mathrm{cm}\end{array}$ & Side \\
\hline 64.9 & frapro & 21.9 & $\mathrm{n}$ \\
\hline 65.8 & plaaqu & 12.0 & $\mathrm{n}$ \\
\hline 65.8 & plaaqu & 12.0 & $\mathrm{n}$ \\
\hline 69.3 & nysaqu & 56.4 & $\mathrm{n}$ \\
\hline 70.2 & \multicolumn{3}{|c|}{ marker \# 98} \\
\hline 76.8 & nysaqu & 51.3 & $\mathrm{n}$ \\
\hline 79.7 & nysaqu & 36.4 & $\mathrm{n}$ \\
\hline 79.9 & taxdis & 19.2 & $\mathrm{n}$ \\
\hline \multirow{2}{*}{82.0} & \multirow{2}{*}{ frapro } & 30.3 & \multirow{2}{*}{$\mathrm{n}$} \\
\hline & & 27.9 & \\
\hline 83.0 & snag & 17.5 & $\mathrm{n}$ \\
\hline 85.1 & nysaqu & 42.0 & $\mathrm{n}$ \\
\hline 85.7 & nysaqu & 16.2 & $\mathrm{n}$ \\
\hline 91.2 & frapro & 25.0 & $\mathrm{n}$ \\
\hline 91.6 & nysaqu & 25.8 & $\mathrm{n}$ \\
\hline 93.5 & nysaqu & 30.4 & $\mathrm{n}$ \\
\hline 96.0 & nysaqu & 59.3 & $\mathrm{n}$ \\
\hline 97.2 & \multicolumn{3}{|c|}{ marker \# 99} \\
\hline 99.7 & fracar & 13.5 & $\mathrm{n}$ \\
\hline 100.3 & nysaqu & 41.6 & $\mathrm{n}$ \\
\hline 102.8 & taxdis & 27.0 & $\mathrm{n}$ \\
\hline 105.8 & plaaqu & 12.5 & $\mathrm{n}$ \\
\hline \multirow{5}{*}{108.3} & \multirow{5}{*}{ plaaqu } & 12.8 & \multirow{5}{*}{$\mathrm{n}$} \\
\hline & & 11.1 & \\
\hline & & 11.5 & \\
\hline & & 13.8 & \\
\hline & & 16.0 & \\
\hline 110.3 & fracar & 10.9 & $\mathrm{n}$ \\
\hline 115.2 & plaaqu & 19.5 & $\mathrm{n}$ \\
\hline 117.9 & \multicolumn{3}{|c|}{ marker \# 100} \\
\hline 118.5 & plaaqu & 17.9 & $\mathrm{n}$ \\
\hline 122.1 & plaaqu & 20.0 & $\mathrm{n}$ \\
\hline 127.9 & frapro & 43.0 & $\mathrm{n}$ \\
\hline 129.8 & liqsty & 19.0 & $\mathrm{n}$ \\
\hline 132.0 & fracar & 10.0 & $\mathrm{n}$ \\
\hline 132.5 & taxdis & 31.2 & $\mathrm{n}$ \\
\hline 134.9 & taxdis & 10.6 & $\mathrm{n}$ \\
\hline 135.3 & nysaqu & 48.9 & $\mathrm{n}$ \\
\hline 136.4 & nysaqu & 24.6 & $\mathrm{n}$ \\
\hline 136.7 & taxdis & 25.3 & $\mathrm{n}$ \\
\hline 138.1 & taxdis & 26.4 & $\mathrm{n}$ \\
\hline 138.8 & plaaqu & 27.0 & $\mathrm{n}$ \\
\hline 139.1 & \multicolumn{3}{|c|}{ marker \# 101} \\
\hline 140.5 & nysaqu & 22.1 & $\mathrm{n}$ \\
\hline
\end{tabular}


E KN: The sampling width of this transect is 5 meters (on the north side of the transect line) from -3.1 to 721.5 and 10 meters (5 meters on either side of the transect line) from 721.5 to 729.3 (Continued)

\begin{tabular}{|c|c|c|c|}
\hline $\begin{array}{l}\text { Distance from } \\
\text { beginning of } \\
\text { transect, in } \mathrm{m}\end{array}$ & $\begin{array}{c}\text { Tree } \\
\text { species }\end{array}$ & $\begin{array}{l}\text { Dbh, } \\
\text { in } \mathrm{cm}\end{array}$ & Side \\
\hline \multirow{2}{*}{150.8} & \multirow{2}{*}{ fracar } & 10.6 & \multirow{2}{*}{$\mathrm{n}$} \\
\hline & & 9.5 & \\
\hline 150.9 & taxdis & 39.6 & $\mathrm{n}$ \\
\hline 154.7 & nysaqu & 50.1 & $\mathrm{n}$ \\
\hline 156.9 & plaaqu & 30.4 & $\mathrm{n}$ \\
\hline 160.3 & \multicolumn{3}{|c|}{ marker \# 102} \\
\hline 160.5 & nysaqu & 56.8 & $\mathrm{n}$ \\
\hline 163.5 & plaaqu & 10.9 & $\mathrm{n}$ \\
\hline \multirow{3}{*}{167.6} & \multirow{3}{*}{ plaaqu } & 11.3 & \multirow{3}{*}{$\mathrm{n}$} \\
\hline & & 9.1 & \\
\hline & & 9.2 & \\
\hline 168.5 & acerub & 27.9 & $\mathrm{n}$ \\
\hline \multirow{2}{*}{170.4} & \multirow{2}{*}{ fracar } & 10.7 & \multirow{2}{*}{$\mathrm{n}$} \\
\hline & & 6.8 & \\
\hline \multirow{2}{*}{170.7} & \multirow{2}{*}{ fracar } & 14.3 & \multirow{2}{*}{$\mathrm{n}$} \\
\hline & & 11.7 & \\
\hline 172.3 & fracar & 10.0 & $\mathrm{n}$ \\
\hline 178.5 & taxdis & 31.0 & $\mathrm{n}$ \\
\hline 179.1 & taxdis & 38.8 & $\mathrm{n}$ \\
\hline \multirow{2}{*}{180.7} & \multirow{2}{*}{ taxdis } & 39.4 & \multirow{2}{*}{$\mathrm{n}$} \\
\hline & & 28.4 & \\
\hline 182.6 & snag & 24.7 & $\mathrm{n}$ \\
\hline \multirow{3}{*}{184.7} & plaaqu & \multirow{3}{*}{22.9} & \multirow{3}{*}{$\mathrm{n}$} \\
\hline & plaaqu & & \\
\hline & plaaqu & & \\
\hline 184.7 & taxdis & 13.0 & $\mathrm{n}$ \\
\hline 186.8 & taxdis & 45.9 & $\mathrm{n}$ \\
\hline 188.2 & fracar & 11.0 & $\mathrm{n}$ \\
\hline 190.8 & \multicolumn{3}{|c|}{ marker \# 103} \\
\hline 191.5 & acerub & 27.4 & $\mathrm{n}$ \\
\hline 192.3 & liqsty & 44.1 & $\mathrm{n}$ \\
\hline 192.4 & taxdis & 17.4 & $\mathrm{n}$ \\
\hline 200.8 & nysaqu & 52.5 & $\mathrm{n}$ \\
\hline 202.3 & taxdis & 38.0 & $\mathrm{n}$ \\
\hline 206.2 & fracar & 10.6 & $\mathrm{n}$ \\
\hline 206.5 & fracar & 10.1 & $\mathrm{n}$ \\
\hline 208.3 & nysaqu & 53.3 & $\mathrm{n}$ \\
\hline 208.7 & snag & 19.7 & $\mathrm{n}$ \\
\hline \multirow{2}{*}{212.3} & \multirow{2}{*}{ plaaqu } & 30.5 & \multirow{2}{*}{$\mathrm{n}$} \\
\hline & & 6.5 & \\
\hline 212.6 & nysaqu & 58.6 & $\mathrm{n}$ \\
\hline 214.0 & fracar & 10.3 & $\mathrm{n}$ \\
\hline 214.0 & fracar & 9.4 & $\mathrm{n}$ \\
\hline 216.0 & taxdis & 65.5 & $\mathrm{n}$ \\
\hline 218.3 & plaaqu & 17.5 & $\mathrm{n}$ \\
\hline 219.2 & nysaqu & 14.6 & $\mathrm{n}$ \\
\hline 221.7 & & $r \# 104$ & \\
\hline
\end{tabular}

\begin{tabular}{|c|c|c|c|}
\hline $\begin{array}{l}\text { Distance from } \\
\text { beginning of } \\
\text { transect, in } \mathrm{m}\end{array}$ & $\begin{array}{c}\text { Tree } \\
\text { species }\end{array}$ & $\begin{array}{l}\text { Dbh, } \\
\text { in } \mathrm{cm}\end{array}$ & Side \\
\hline 230.4 & nysaqu & 32.0 & $\mathrm{n}$ \\
\hline 234.6 & nysaqu & 31.8 & $\mathrm{n}$ \\
\hline 234.6 & nysaqu & 17.9 & $\mathrm{n}$ \\
\hline 237.8 & nysaqu & 61.9 & $\mathrm{n}$ \\
\hline 240.9 & nysaqu & 12.3 & $\mathrm{n}$ \\
\hline 241.4 & nysaqu & 21.4 & $\mathrm{n}$ \\
\hline 241.4 & nysaqu & 23.1 & $\mathrm{n}$ \\
\hline 242.2 & taxdis & 39.0 & $\mathrm{n}$ \\
\hline 242.4 & nysaqu & 38.0 & $\mathrm{n}$ \\
\hline 242.5 & nysaqu & 44.4 & $\mathrm{n}$ \\
\hline 242.8 & nysaqu & 16.7 & $\mathrm{n}$ \\
\hline 243.8 & nysaqu & 25.8 & $\mathrm{n}$ \\
\hline 246.2 & taxdis & 13.8 & $\mathrm{n}$ \\
\hline 247.2 & taxdis & 23.3 & $\mathrm{n}$ \\
\hline 248.1 & taxdis & 40.4 & $\mathrm{n}$ \\
\hline 250.4 & taxdis & 41.5 & $\mathrm{n}$ \\
\hline 257.1 & nysaqu & 35.3 & $\mathrm{n}$ \\
\hline 257.5 & taxdis & 39.6 & $\mathrm{n}$ \\
\hline 260.0 & nysaqu & 16.9 & $\mathrm{n}$ \\
\hline 260.1 & taxdis & 46.0 & $\mathrm{n}$ \\
\hline 265.2 & \multicolumn{3}{|c|}{ marker \# 105} \\
\hline \multirow{2}{*}{265.7} & \multirow{2}{*}{ nysaqu } & 48.5 & \multirow{2}{*}{$\mathrm{n}$} \\
\hline & & 39.2 & \\
\hline 269.0 & taxdis & 43.0 & $\mathrm{n}$ \\
\hline \multirow{2}{*}{273.3} & \multirow{2}{*}{ fracar } & 15.2 & \multirow{2}{*}{$\mathrm{n}$} \\
\hline & & 5.0 & \\
\hline 273.7 & snag & 12.5 & $\mathrm{n}$ \\
\hline 277.5 & ulmame & 26.9 & $\mathrm{n}$ \\
\hline 281.2 & taxdis & 23.4 & $\mathrm{n}$ \\
\hline \multirow{5}{*}{282.8} & \multirow{5}{*}{ plaaqu } & 16.7 & \multirow{5}{*}{$\mathrm{n}$} \\
\hline & & 14.8 & \\
\hline & & 9.0 & \\
\hline & & 6.0 & \\
\hline & & 16.9 & \\
\hline 283.3 & ulmame & 30.3 & $\mathrm{n}$ \\
\hline 290.3 & betnig & 15.5 & $\mathrm{n}$ \\
\hline 290.2 & \multicolumn{3}{|c|}{ marker \# 106} \\
\hline 295.9 & caraqu & 60.6 & $\mathrm{n}$ \\
\hline 302.6 & quelau & 65.9 & $\mathrm{n}$ \\
\hline 306.0 & taxdis & 16.9 & $\mathrm{n}$ \\
\hline 306.1 & quelau & 21.4 & $\mathrm{n}$ \\
\hline 308.1 & cravir & 13.5 & $\mathrm{n}$ \\
\hline 314.9 & cravir & 11.8 & $\mathrm{n}$ \\
\hline 315.4 & caraqu & 14.8 & $\mathrm{n}$ \\
\hline 316.2 & quelau & 35.8 & $\mathrm{n}$ \\
\hline 321.5 & liqsty & 33.4 & $\mathrm{n}$ \\
\hline 322.4 & carcar & 16.5 & $\mathrm{n}$ \\
\hline
\end{tabular}


E KN: The sampling width of this transect is 5 meters (on the north side of the transect line) from -3.1 to 721.5 and 10 meters (5 meters on either side of the transect line) from 721.5 to 729.3 (Continued)

\begin{tabular}{|c|c|c|c|c|c|c|c|}
\hline $\begin{array}{l}\text { Distance from } \\
\text { beginning of } \\
\text { transect, in } m\end{array}$ & $\begin{array}{c}\text { Tree } \\
\text { species }\end{array}$ & $\begin{array}{l}\text { Dbh, } \\
\text { in cm }\end{array}$ & Side & $\begin{array}{l}\text { Distance from } \\
\text { beginning of } \\
\text { transect, in m }\end{array}$ & $\begin{array}{c}\text { Tree } \\
\text { species }\end{array}$ & $\begin{array}{l}\text { Dbh, } \\
\text { in } \mathrm{cm}\end{array}$ & Side \\
\hline 322.6 & \multicolumn{3}{|c|}{ marker \# 107} & 405.8 & taxdis & 24.3 & $\mathrm{n}$ \\
\hline \multirow{2}{*}{322.6} & \multirow{2}{*}{ liqsty } & 45.3 & \multirow{2}{*}{$\mathrm{n}$} & 406.9 & taxdis & 21.9 & $\mathrm{n}$ \\
\hline & & 37.4 & & 407.0 & taxdis & 30.8 & $\mathrm{n}$ \\
\hline 323.0 & liqsty & 19.9 & $\mathrm{n}$ & 407.1 & taxdis & 17.2 & $\mathrm{n}$ \\
\hline 325.8 & taxdis & 14.4 & $\mathrm{n}$ & 407.6 & \multicolumn{3}{|c|}{ marker \# 110} \\
\hline 327.5 & betnig & 14.7 & $\mathrm{n}$ & 408.1 & taxdis & 30.3 & $\mathrm{n}$ \\
\hline 334.7 & plaaqu & 22.0 & $\mathrm{n}$ & 408.1 & taxdis & 20.5 & $\mathrm{n}$ \\
\hline 335.2 & plaaqu & 14.1 & $\mathrm{n}$ & 408.6 & taxdis & 43.5 & $\mathrm{n}$ \\
\hline 335.5 & nysaqu & 25.8 & $\mathrm{n}$ & 411.7 & taxdis & 25.2 & $\mathrm{n}$ \\
\hline 339.5 & nysaqu & 44.0 & $\mathrm{n}$ & 413.5 & taxdis & 14.3 & $\mathrm{n}$ \\
\hline 343.6 & fracar & 10.5 & $\mathrm{n}$ & 413.6 & taxdis & 31.5 & $\mathrm{n}$ \\
\hline 344.5 & fracar & 11.9 & $\mathrm{n}$ & 414.1 & taxdis & 21.5 & $\mathrm{n}$ \\
\hline 344.9 & snag & 10.4 & $\mathrm{n}$ & \multirow{3}{*}{415.8} & \multirow{3}{*}{ fracar } & 10.9 & \multirow{3}{*}{$\mathrm{n}$} \\
\hline 345.7 & cepocc & 10.1 & $\mathrm{n}$ & & & 6.3 & \\
\hline 348.5 & & r\# 108 & & & & 8.6 & \\
\hline 349.5 & taxdis & 53.6 & $\mathrm{n}$ & 415.8 & taxdis & 24.3 & $\mathrm{n}$ \\
\hline 349.7 & nysaqu & 12.8 & $\mathrm{n}$ & 416.4 & taxdis & 31.5 & $\mathrm{n}$ \\
\hline 353.4 & taxdis & 22.3 & $\mathrm{n}$ & 416.8 & fracar & 11.0 & $\mathrm{n}$ \\
\hline \multirow{2}{*}{357.3} & \multirow{2}{*}{ plaaqu } & 22.5 & \multirow{2}{*}{$\mathrm{n}$} & 417.4 & taxdis & 30.0 & $\mathrm{n}$ \\
\hline & & 8.6 & & 417.6 & taxdis & 46.9 & $\mathrm{n}$ \\
\hline 358.5 & plaaqu & 23.5 & $\mathrm{n}$ & 419.2 & snag & 10.3 & $\mathrm{n}$ \\
\hline 360.9 & frapro & 39.9 & $\mathrm{n}$ & 420.1 & fracar & 10.9 & $\mathrm{n}$ \\
\hline 361.5 & fracar & 20.8 & $\mathrm{n}$ & 420.4 & fracar & 10.7 & $\mathrm{n}$ \\
\hline 361.5 & fracar & 16.2 & $\mathrm{n}$ & 422.5 & plaaqu & 21.9 & $\mathrm{n}$ \\
\hline \multirow{2}{*}{370.0} & \multirow{2}{*}{ nysaqu } & 45.0 & \multirow{2}{*}{$\mathrm{n}$} & 422.9 & liqsty & 17.8 & $\mathrm{n}$ \\
\hline & & 22.7 & & \multirow{2}{*}{423.2} & \multirow{2}{*}{ fracar } & 12.0 & \multirow{2}{*}{$\mathrm{n}$} \\
\hline 371.6 & nysaqu & 51.0 & $\mathrm{n}$ & & & 4.5 & \\
\hline 373.4 & nysbif & 51.5 & $\mathrm{n}$ & 425.9 & frapro & 40.8 & $\mathrm{n}$ \\
\hline 373.8 & plaaqu & 16.4 & $\mathrm{n}$ & 426.3 & plaaqu & 10.8 & $\mathrm{n}$ \\
\hline 376.2 & taxdis & 54.8 & $\mathrm{n}$ & 428.0 & ulmame & 11.0 & $\mathrm{n}$ \\
\hline 382.8 & frapro & 23.7 & $\mathrm{n}$ & 431.3 & & \# 111 & \\
\hline 383.3 & \multicolumn{3}{|c|}{ marker \# 109} & \multirow{3}{*}{437.0} & \multirow{3}{*}{ fracar } & 13.1 & \multirow{3}{*}{$\mathrm{n}$} \\
\hline 383.3 & nysaqu & 32.4 & $\mathrm{n}$ & & & 4.8 & \\
\hline 383.3 & nysaqu & 40.3 & $\mathrm{n}$ & & & 8.8 & \\
\hline 389.6 & taxdis & 40.5 & $\mathrm{n}$ & 438.4 & snag & 11.0 & $\mathrm{n}$ \\
\hline 390.8 & taxdis & 45.9 & $\mathrm{n}$ & 439.7 & ilecas & 11.3 & $\mathrm{n}$ \\
\hline 390.9 & taxdis & 26.5 & $\mathrm{n}$ & 440.2 & taxdis & 39.4 & $\mathrm{n}$ \\
\hline 391.3 & liqsty & 16.5 & $\mathrm{n}$ & 442.8 & nysaqu & 49.7 & $\mathrm{n}$ \\
\hline 392.2 & plaaqu & 10.1 & $\mathrm{n}$ & 444.4 & taxdis & 34.9 & $\mathrm{n}$ \\
\hline 394.8 & taxdis & 30.8 & $\mathrm{n}$ & 445.6 & taxdis & 24.6 & $\mathrm{n}$ \\
\hline 396.4 & taxdis & 31.2 & $\mathrm{n}$ & 447.0 & taxdis & 45.2 & $\mathrm{n}$ \\
\hline 397.6 & fracar & 10.5 & $\mathrm{n}$ & 452.3 & nysaqu & 36.2 & $\mathrm{n}$ \\
\hline 399.6 & taxdis & 23.5 & $\mathrm{n}$ & 452.6 & & \# \# 112 & \\
\hline 400.9 & nysaqu & 54.7 & $\mathrm{n}$ & 453.6 & nysaqu & 40.3 & $\mathrm{n}$ \\
\hline 401.5 & taxdis & 18.1 & $\mathrm{n}$ & 453.9 & nysaqu & 44.4 & $\mathrm{n}$ \\
\hline 403.6 & taxdis & 34.0 & $\mathrm{n}$ & 453.9 & snag & 17.1 & $\mathrm{n}$ \\
\hline 404.5 & nysaqu & 43.6 & $\mathrm{n}$ & 455.6 & nysaqu & 40.8 & $\mathrm{n}$ \\
\hline
\end{tabular}


E KN: The sampling width of this transect is 5 meters (on the north side of the transect line) from -3.1 to 721.5 and 10 meters (5 meters on either side of the transect line) from 721.5 to 729.3 (Continued)

\begin{tabular}{|c|c|c|c|}
\hline $\begin{array}{l}\text { Distance from } \\
\text { beginning of } \\
\text { transect, in } \mathrm{m}\end{array}$ & $\begin{array}{c}\text { Tree } \\
\text { species }\end{array}$ & $\begin{array}{l}\text { Dbh, } \\
\text { in cm }\end{array}$ & Side \\
\hline 457.7 & taxdis & 42.0 & $\mathrm{n}$ \\
\hline 459.0 & taxdis & 29.2 & $\mathrm{n}$ \\
\hline \multirow{3}{*}{460.7} & \multirow{3}{*}{ fracar } & 11.6 & \multirow{3}{*}{$\mathrm{n}$} \\
\hline & & 7.5 & \\
\hline & & 9.0 & \\
\hline 460.9 & nysaqu & 38.1 & $\mathrm{n}$ \\
\hline 461.0 & taxdis & 35.9 & $\mathrm{n}$ \\
\hline 464.5 & taxdis & 21.5 & $\mathrm{n}$ \\
\hline \multirow{2}{*}{465.3} & \multirow{2}{*}{ snag } & 22.8 & \multirow{2}{*}{$\mathrm{n}$} \\
\hline & & 19.9 & \\
\hline 465.6 & taxdis & 34.3 & $\mathrm{n}$ \\
\hline 468.5 & liqsty & 16.7 & $\mathrm{n}$ \\
\hline 470.5 & taxdis & 14.6 & $\mathrm{n}$ \\
\hline 471.2 & taxdis & 15.4 & $\mathrm{n}$ \\
\hline 477.3 & nysaqu & 35.1 & $\mathrm{n}$ \\
\hline 477.5 & frapro & 14.7 & $\mathrm{n}$ \\
\hline 481.0 & \multicolumn{3}{|c|}{ marker \# 113} \\
\hline 481.8 & taxdis & 42.5 & $\mathrm{n}$ \\
\hline 481.8 & taxdis & 13.4 & $\mathrm{n}$ \\
\hline 482.9 & nysaqu & 52.4 & $\mathrm{n}$ \\
\hline 487.5 & liqsty & 50.2 & $\mathrm{n}$ \\
\hline 489.2 & fracar & 18.0 & $\mathrm{n}$ \\
\hline 490.5 & fracar & 16.7 & $\mathrm{n}$ \\
\hline 491.9 & taxdis & 20.4 & $\mathrm{n}$ \\
\hline 496.6 & taxdis & 20.2 & $\mathrm{n}$ \\
\hline 497.1 & caraqu & 28.0 & $\mathrm{n}$ \\
\hline 498.4 & nysaqu & 42.0 & $\mathrm{n}$ \\
\hline 504.3 & taxdis & 49.2 & $\mathrm{n}$ \\
\hline 507.7 & taxdis & 16.3 & $\mathrm{n}$ \\
\hline 511.2 & taxdis & 15.4 & $\mathrm{n}$ \\
\hline 511.7 & liqsty & 20.4 & $\mathrm{n}$ \\
\hline 512.1 & taxdis & 40.6 & $\mathrm{n}$ \\
\hline 512.3 & \multicolumn{3}{|c|}{ marker \# 114} \\
\hline \multirow{2}{*}{514.4} & \multirow{2}{*}{ frapro } & 16.8 & \multirow{2}{*}{$\mathrm{n}$} \\
\hline & & 20.1 & \\
\hline 515.0 & frapro & 15.6 & $\mathrm{n}$ \\
\hline 524.3 & nysaqu & 57.2 & $\mathrm{n}$ \\
\hline 526.7 & nysaqu & 22.9 & $\mathrm{n}$ \\
\hline 527.9 & ulmame & 22.1 & $\mathrm{n}$ \\
\hline 528.9 & nysaqu & 39.0 & $\mathrm{n}$ \\
\hline 531.5 & nysaqu & 51.1 & $\mathrm{n}$ \\
\hline 532.3 & nysaqu & 32.8 & $\mathrm{n}$ \\
\hline 534.2 & taxdis & 49.3 & $\mathrm{n}$ \\
\hline 540.1 & nysaqu & 49.4 & $\mathrm{n}$ \\
\hline \multirow{2}{*}{545.2} & \multirow{2}{*}{ fracar } & 10.6 & \multirow{2}{*}{$\mathrm{n}$} \\
\hline & & 4.0 & \\
\hline 548.9 & plaaqu & 15.3 & $\mathrm{n}$ \\
\hline
\end{tabular}

\begin{tabular}{|c|c|c|c|}
\hline $\begin{array}{l}\text { Distance from } \\
\text { beginning of } \\
\text { transect, in } \mathrm{m}\end{array}$ & $\begin{array}{c}\text { Tree } \\
\text { species }\end{array}$ & $\begin{array}{l}\text { Dbh, } \\
\text { in cm }\end{array}$ & Side \\
\hline 550.3 & \multicolumn{3}{|c|}{ marker \# 115} \\
\hline 555.6 & taxdis & 28.5 & $\mathrm{n}$ \\
\hline 556.7 & taxdis & 24.5 & $\mathrm{n}$ \\
\hline 557.8 & taxdis & 31.6 & $\mathrm{n}$ \\
\hline 558.3 & taxdis & 28.5 & $\mathrm{n}$ \\
\hline 559.0 & taxdis & 25.9 & $\mathrm{n}$ \\
\hline 559.5 & taxdis & 14.9 & $\mathrm{n}$ \\
\hline 560.2 & liqsty & 10.6 & $\mathrm{n}$ \\
\hline 561.4 & taxdis & 12.3 & $\mathrm{n}$ \\
\hline 561.4 & taxdis & 26.9 & $\mathrm{n}$ \\
\hline 561.9 & taxdis & 50.8 & $\mathrm{n}$ \\
\hline 564.0 & fracar & 12.5 & $\mathrm{n}$ \\
\hline 564.5 & frapro & 20.4 & $\mathrm{n}$ \\
\hline 564.5 & frapro & 22.4 & $\mathrm{n}$ \\
\hline 567.2 & nysaqu & 41.5 & $\mathrm{n}$ \\
\hline \multirow{2}{*}{573.2} & \multirow{2}{*}{ nysaqu } & 34.6 & \multirow{2}{*}{$\mathrm{n}$} \\
\hline & & 42.0 & \\
\hline 574.3 & taxdis & 24.0 & $\mathrm{n}$ \\
\hline 575.7 & fracar & 15.2 & $\mathrm{n}$ \\
\hline 580.6 & \multicolumn{3}{|c|}{ marker \# 116} \\
\hline 584.9 & nysaqu & 13.7 & $\mathrm{n}$ \\
\hline \multirow{2}{*}{586.6} & \multirow{2}{*}{ fracar } & 10.7 & \multirow{2}{*}{$\mathrm{n}$} \\
\hline & & 6.8 & \\
\hline 587.3 & nysaqu & 24.4 & $\mathrm{n}$ \\
\hline \multirow{2}{*}{595.1} & \multirow{2}{*}{ frapro } & 23.3 & \multirow{2}{*}{$\mathrm{n}$} \\
\hline & & 39.1 & \\
\hline 599.1 & fracar & 10.6 & $\mathrm{n}$ \\
\hline 600.5 & taxdis & 56.0 & $\mathrm{n}$ \\
\hline 605.5 & nysaqu & 34.6 & $\mathrm{n}$ \\
\hline 607.4 & taxdis & 20.0 & $\mathrm{n}$ \\
\hline 607.5 & \multicolumn{3}{|c|}{ marker \# 117} \\
\hline 609.8 & nysaqu & 47.6 & $\mathrm{n}$ \\
\hline 611.8 & taxdis & 20.4 & $\mathrm{n}$ \\
\hline 612.3 & taxdis & 46.7 & $\mathrm{n}$ \\
\hline 617.0 & nysaqu & 21.3 & $\mathrm{n}$ \\
\hline 618.0 & taxdis & 40.5 & $\mathrm{n}$ \\
\hline 622.8 & taxdis & 33.4 & $\mathrm{n}$ \\
\hline 624.0 & nysaqu & 24.2 & $\mathrm{n}$ \\
\hline 625.8 & fracar & 15.0 & $\mathrm{n}$ \\
\hline 626.2 & taxdis & 31.8 & $\mathrm{n}$ \\
\hline 626.9 & taxdis & 20.9 & $\mathrm{n}$ \\
\hline 630.5 & \multicolumn{3}{|c|}{ marker \# 118} \\
\hline 638.6 & fracar & 10.1 & $\mathrm{n}$ \\
\hline 642.0 & caraqu & 10.0 & $\mathrm{n}$ \\
\hline 642.3 & taxdis & 33.0 & $\mathrm{n}$ \\
\hline 644.9 & taxdis & 47.0 & $\mathrm{n}$ \\
\hline 645.4 & snag & 17.4 & $\mathrm{n}$ \\
\hline
\end{tabular}


E KN: The sampling width of this transect is 5 meters (on the north side of the transect line) from -3.1 to 721.5 and 10 meters (5 meters on either side of the transect line) from 721.5 to 729.3 (Continued)

\begin{tabular}{|c|c|c|c|c|c|c|c|}
\hline $\begin{array}{l}\text { Distance from } \\
\text { beginning of } \\
\text { transect, in } \mathrm{m}\end{array}$ & $\begin{array}{c}\text { Tree } \\
\text { species }\end{array}$ & $\begin{array}{l}\text { Dbh, } \\
\text { in cm }\end{array}$ & Side & $\begin{array}{l}\text { Distance from } \\
\text { beginning of } \\
\text { transect, in } \mathrm{m}\end{array}$ & $\begin{array}{c}\text { Tree } \\
\text { species }\end{array}$ & $\begin{array}{l}\text { Dbh, } \\
\text { in cm }\end{array}$ & Side \\
\hline 645.7 & frapro & 23.4 & $\mathrm{n}$ & 732.4 & caraqu & 52.6 & $\mathrm{~s}$ \\
\hline \multirow{2}{*}{647.4} & fracar & \multirow{2}{*}{14.5} & \multirow{2}{*}{$\mathrm{n}$} & \multirow{5}{*}{733.6} & \multirow{5}{*}{ acerub } & 37.4 & \multirow{5}{*}{$\mathrm{n}$} \\
\hline & fracar & & & & & 22.2 & \\
\hline 647.9 & taxdis & 27.4 & $\mathrm{n}$ & & & 7.6 & \\
\hline 650.3 & taxdis & 24.4 & $\mathrm{n}$ & & & 16.5 & \\
\hline 652.7 & nysaqu & 57.3 & $\mathrm{n}$ & & & 5.9 & \\
\hline 653.8 & \multicolumn{3}{|c|}{ marker \# 119} & 733.9 & nysaqu & 54.2 & $\mathrm{n}$ \\
\hline 656.7 & taxdis & 41.3 & $\mathrm{n}$ & & & & \\
\hline 662.1 & taxdis & 26.4 & $\mathrm{n}$ & & & & \\
\hline 664.0 & nysaqu & 43.5 & $\mathrm{n}$ & & & & \\
\hline \multirow{2}{*}{666.2} & \multirow{2}{*}{ fracar } & 15.8 & \multirow{2}{*}{$\mathrm{n}$} & & & & \\
\hline & & 6.4 & & & & & \\
\hline 667.5 & fracar & 11.3 & $\mathrm{n}$ & & & & \\
\hline \multirow{5}{*}{672.8} & \multirow{5}{*}{ fracar } & 9.1 & \multirow{5}{*}{$\mathrm{n}$} & & & & \\
\hline & & 5.5 & & & & & \\
\hline & & 12.2 & & & & & \\
\hline & & 8.5 & & & & & \\
\hline & & 7.0 & & & & & \\
\hline 673.1 & fracar & 13.2 & $\mathrm{n}$ & & & & \\
\hline 679.2 & \multicolumn{3}{|c|}{ marker \# 120} & & & & \\
\hline 682.1 & plaaqu & 12.3 & $\mathrm{n}$ & & & & \\
\hline 685.3 & plaaqu & 13.3 & $\mathrm{n}$ & & & & \\
\hline 687.8 & frapro & 37.4 & $\mathrm{n}$ & & & & \\
\hline 688.7 & frapro & 14.3 & $\mathrm{n}$ & & & & \\
\hline 689.7 & ulmame & 10.0 & $\mathrm{n}$ & & & & \\
\hline 691.8 & taxdis & 91.4 & $\mathrm{n}$ & & & & \\
\hline 693.0 & nysaqu & 61.8 & $\mathrm{n}$ & & & & \\
\hline 694.2 & fracar & 14.0 & $\mathrm{n}$ & & & & \\
\hline 698.4 & taxdis & 30.4 & $\mathrm{n}$ & & & & \\
\hline 698.8 & taxdis & 28.7 & $\mathrm{n}$ & & & & \\
\hline \multirow{8}{*}{727.7} & \multirow{8}{*}{ fracar } & 11.4 & \multirow{8}{*}{$\mathrm{s}$} & & & & \\
\hline & & 6.2 & & & & & \\
\hline & & 12.6 & & & & & \\
\hline & & 9.0 & & & & & \\
\hline & & 4.3 & & & & & \\
\hline & & 8.3 & & & & & \\
\hline & & 8.4 & & & & & \\
\hline & & 10.2 & & & & & \\
\hline 729.3 & \multicolumn{3}{|c|}{ marker \# 121} & & & & \\
\hline 731.3 & betnig & 32.1 & $\mathrm{n}$ & & & & \\
\hline 731.4 & frapro & 48.3 & $\mathrm{~s}$ & & & & \\
\hline \multirow{2}{*}{732.3} & \multirow{2}{*}{ fracar } & 12.7 & \multirow{2}{*}{$\mathrm{n}$} & & & & \\
\hline & & 6.0 & & & & & \\
\hline 732.3 & taxdis & 52.4 & $\mathrm{n}$ & & & & \\
\hline \multirow{2}{*}{732.4} & & 13.7 & & & & & \\
\hline & fracar & 11.3 & $\mathrm{n}$ & & & & \\
\hline
\end{tabular}


F KI: The sampling width of this transect is 10 meters (5 meters on either side of the transect line).

\begin{tabular}{|c|c|c|c|}
\hline $\begin{array}{l}\text { Distance from } \\
\text { beginning of } \\
\text { transect, in } m\end{array}$ & $\begin{array}{c}\text { Tree } \\
\text { species }\end{array}$ & $\begin{array}{l}\text { Dbh, } \\
\text { in cm }\end{array}$ & Side \\
\hline 0.0 & \multicolumn{3}{|c|}{ marker \# 122} \\
\hline 3.6 & liqsty & 47.2 & $\mathrm{w}$ \\
\hline 7.9 & quelau & 54.0 & w \\
\hline 9.6 & quelau & 24.6 & w \\
\hline 11.4 & liqsty & 32.5 & $\mathrm{e}$ \\
\hline 16.9 & caraqu & 23.2 & w \\
\hline 17.8 & carcar & 11.4 & $\mathrm{e}$ \\
\hline \multirow{2}{*}{22.8} & \multirow{2}{*}{ betnig } & 37.8 & \multirow{2}{*}{ w } \\
\hline & & 6.3 & \\
\hline \multirow{2}{*}{24.2} & \multirow{2}{*}{ betnig } & 18.6 & \multirow{2}{*}{$\mathrm{w}$} \\
\hline & & 9.5 & \\
\hline 26.9 & betnig & 21.0 & $\mathrm{w}$ \\
\hline 26.9 & sabpal & 19.3 & $\mathrm{w}$ \\
\hline 28.4 & sabpal & 26.8 & $\mathrm{e}$ \\
\hline 36.9 & \multicolumn{3}{|c|}{ marker \# 123} \\
\hline 39.6 & betnig & 18.4 & $\mathrm{w}$ \\
\hline 40.1 & sabpal & 26.8 & w \\
\hline 41.4 & carcar & 14.9 & $\mathrm{e}$ \\
\hline 43.3 & carcar & 16.5 & w \\
\hline 45.6 & betnig & 37.6 & w \\
\hline 46.6 & liqsty & 18.0 & $\mathrm{e}$ \\
\hline 54.4 & liqsty & 12.0 & $\mathrm{e}$ \\
\hline 54.6 & liqsty & 10.9 & $\mathrm{w}$ \\
\hline 56.5 & liqsty & 11.0 & w \\
\hline 56.9 & cravir & 16.4 & w \\
\hline 62.3 & carcar & 20.4 & $\mathrm{e}$ \\
\hline 64.2 & betnig & 19.6 & w \\
\hline 67.7 & carcar & 11.4 & $\mathrm{e}$ \\
\hline 73.8 & carcar & 15.3 & $\mathrm{w}$ \\
\hline 74.0 & \multicolumn{3}{|c|}{ marker \# 124} \\
\hline 77.1 & carcar & 20.3 & $\mathrm{e}$ \\
\hline 77.5 & quelau & 53.8 & $\mathrm{e}$ \\
\hline 81.8 & quelau & 37.0 & $\mathrm{e}$ \\
\hline 83.9 & quelau & 52.4 & w \\
\hline 87.3 & betnig & 19.9 & $\mathrm{e}$ \\
\hline 94.8 & liqsty & 32.5 & w \\
\hline 95.8 & betnig & 13.2 & $\mathrm{e}$ \\
\hline 100.0 & \multicolumn{3}{|c|}{ marker \# 125} \\
\hline
\end{tabular}


G TK: The sampling width of this transect is 5 meters on the west side of the transect line

\begin{tabular}{|c|c|c|c|c|c|c|c|}
\hline $\begin{array}{l}\text { Distance from } \\
\text { beginning of } \\
\text { transect, in } \mathrm{m}\end{array}$ & $\begin{array}{c}\text { Tree } \\
\text { species }\end{array}$ & $\begin{array}{l}\text { Dbh, } \\
\text { in } \mathbf{c m}\end{array}$ & Side & $\begin{array}{l}\text { Distance from } \\
\text { beginning of } \\
\text { transect, in } \mathrm{m}\end{array}$ & $\begin{array}{c}\text { Tree } \\
\text { species }\end{array}$ & $\begin{array}{l}\text { Dbh, } \\
\text { in cm }\end{array}$ & Side \\
\hline 0.0 & \multicolumn{3}{|c|}{ marker \# 126} & 66.5 & nysbif & 28.5 & $\mathrm{w}$ \\
\hline 3.3 & snag & 11.5 & $\mathrm{w}$ & 67.6 & nysbif & 13.0 & $\mathrm{w}$ \\
\hline 3.6 & quelau & 11.2 & $\mathrm{w}$ & 68.9 & snag & 18.4 & $\mathrm{w}$ \\
\hline 4.8 & liqsty & 37.6 & $\mathrm{w}$ & 69.7 & nysbif & 35.9 & $\mathrm{w}$ \\
\hline 7.5 & taxdis & 29.9 & $\mathrm{w}$ & 70.0 & nysbif & 34.5 & $\mathrm{w}$ \\
\hline 10.5 & sabpal & 28.6 & $\mathrm{w}$ & 70.1 & nysaqu & 10.0 & w \\
\hline 11.0 & quelau & 48.1 & $\mathrm{w}$ & 70.3 & frapro & 17.6 & $\mathrm{w}$ \\
\hline 15.1 & nysbif & 15.2 & $\mathrm{w}$ & 75.7 & nysbif & 20.3 & $\mathrm{w}$ \\
\hline 15.9 & sabpal & 18.7 & $\mathrm{w}$ & 76.3 & nysbif & 31.8 & $\mathrm{w}$ \\
\hline 16.4 & liqsty & 16.7 & $\mathrm{w}$ & 77.9 & frapro & 36.6 & $\mathrm{w}$ \\
\hline 20.8 & taxdis & 15.8 & $\mathrm{w}$ & 81.4 & nysbif & 29.6 & $\mathrm{w}$ \\
\hline 23.2 & nysbif & 33.4 & $\mathrm{w}$ & 83.8 & snag & 19.5 & w \\
\hline 23.8 & acerub & 10.4 & $\mathrm{w}$ & 84.3 & \multicolumn{3}{|c|}{ marker \#129 } \\
\hline 25.9 & taxdis & 11.5 & $\mathrm{w}$ & 87.2 & nysaqu & 44.5 & $\mathrm{w}$ \\
\hline 29.6 & \multicolumn{3}{|c|}{ marker \# 127} & 87.4 & acerub & 19.4 & $\mathrm{w}$ \\
\hline 30.1 & taxdis & 24.0 & $\mathrm{w}$ & 89.1 & frapro & 32.0 & $\mathrm{w}$ \\
\hline 30.8 & nysbif & 25.0 & $\mathrm{w}$ & 91.0 & nysaqu & 56.9 & $\mathrm{w}$ \\
\hline 31.1 & nysbif & 19.5 & $\mathrm{w}$ & 93.2 & frapro & 12.8 & $\mathrm{w}$ \\
\hline 31.4 & frapro & 11.2 & $\mathrm{w}$ & 94.4 & nysaqu & 29.7 & w \\
\hline 32.3 & taxdis & 24.0 & $\mathrm{w}$ & 96.5 & taxdis & 11.9 & $\mathrm{w}$ \\
\hline 34.1 & nysbif & 41.3 & w & 100.3 & taxdis & 10.2 & $\mathrm{w}$ \\
\hline 35.5 & taxdis & 27.6 & $\mathrm{w}$ & 101.5 & nysbif & 64.5 & $\mathrm{w}$ \\
\hline 35.6 & nysbif & 35.1 & $\mathrm{w}$ & 105.2 & taxdis & 13.0 & $\mathrm{w}$ \\
\hline 36.3 & nysbif & 11.3 & $\mathrm{w}$ & 105.7 & frapro & 37.7 & $\mathrm{w}$ \\
\hline 36.5 & nysbif & 21.0 & $\mathrm{w}$ & 107.9 & taxdis & 26.1 & $\mathrm{w}$ \\
\hline 38.1 & nysbif & 31.0 & $\mathrm{w}$ & 108.6 & taxdis & 30.6 & $\mathrm{w}$ \\
\hline 40.1 & liqsty & 39.8 & $\mathrm{w}$ & 108.8 & taxdis & 14.0 & $\mathrm{w}$ \\
\hline 42.1 & nysbif & 24.5 & $\mathrm{w}$ & 110.5 & \multicolumn{3}{|c|}{ marker \#130 } \\
\hline 43.2 & nysbif & 27.0 & $\mathrm{w}$ & 111.8 & taxdis & 26.7 & w \\
\hline 43.3 & nysbif & 37.8 & $\mathrm{w}$ & 111.9 & taxdis & 30.8 & $\mathrm{w}$ \\
\hline 43.6 & nysbif & 13.3 & $\mathrm{w}$ & 114.2 & taxdis & 26.1 & $\mathrm{w}$ \\
\hline 44.1 & nysbif & 22.5 & $\mathrm{w}$ & 115.1 & liqsty & 33.0 & $\mathrm{w}$ \\
\hline 44.4 & ulmame & 18.2 & $\mathrm{w}$ & 119.2 & acerub & 21.1 & w \\
\hline 47.6 & sabpal & 23.5 & $\mathrm{w}$ & 119.8 & taxdis & 42.0 & $\mathrm{w}$ \\
\hline 47.7 & liqsty & 49.7 & $\mathrm{w}$ & 121.5 & taxdis & 19.4 & $\mathrm{w}$ \\
\hline 52.4 & taxdis & 22.4 & $\mathrm{w}$ & 123.4 & taxdis & 45.9 & $\mathrm{w}$ \\
\hline 53.4 & taxdis & 14.3 & $\mathrm{w}$ & 127.2 & taxdis & 24.0 & $\mathrm{w}$ \\
\hline 53.6 & nysbif & 18.9 & $\mathrm{w}$ & 127.6 & nysbif & 15.0 & $\mathrm{w}$ \\
\hline 54.6 & \multicolumn{3}{|c|}{ marker \# 128} & 135.3 & nysbif & 13.8 & w \\
\hline 57.1 & nysbif & 29.0 & $\mathrm{w}$ & 136.3 & snag & 29.8 & $\mathrm{w}$ \\
\hline 60.5 & frapro & 11.3 & $\mathrm{w}$ & 138.0 & liqsty & 12.7 & w \\
\hline 61.0 & nysbif & 11.4 & $\mathrm{w}$ & 140.2 & snag & 59.8 & $\mathrm{w}$ \\
\hline 61.7 & nysaqu & 66.0 & $\mathrm{w}$ & 140.4 & \multicolumn{3}{|c|}{ marker \#131 } \\
\hline 62.4 & nysbif & 38.2 & $\mathrm{w}$ & 141.4 & nysbif & 22.5 & w \\
\hline 65.4 & taxdis & 37.5 & $\mathrm{w}$ & 142.0 & nysbif & 15.3 & $\mathrm{w}$ \\
\hline 66.3 & taxdis & 13.0 & $\mathrm{w}$ & 143.0 & nysbif & 30.5 & $\mathrm{w}$ \\
\hline
\end{tabular}


G TK: The sampling width of this transect is 5 meters on the west side of the transect line

\begin{tabular}{|c|c|c|c|}
\hline $\begin{array}{l}\text { Distance from } \\
\text { beginning of } \\
\text { transect, in } \mathrm{m}\end{array}$ & $\begin{array}{c}\text { Tree } \\
\text { species }\end{array}$ & $\begin{array}{l}\text { Dbh, } \\
\text { in cm }\end{array}$ & Side \\
\hline 145.7 & taxdis & 19.8 & $\mathrm{w}$ \\
\hline 148.4 & nysbif & 35.5 & $\mathrm{w}$ \\
\hline 153.6 & ulmame & 12.0 & $\mathrm{w}$ \\
\hline 156.3 & carcar & 15.4 & $\mathrm{w}$ \\
\hline 158.1 & frapro & 52.9 & $\mathrm{w}$ \\
\hline 163.8 & nysbif & 22.5 & $\mathrm{w}$ \\
\hline 164.9 & nysbif & 10.4 & w \\
\hline \multirow{2}{*}{166.5} & \multirow{2}{*}{ frapro } & 23.8 & \multirow{2}{*}{$\mathrm{w}$} \\
\hline & & 5.2 & \\
\hline 166.8 & frapro & 51.8 & $\mathrm{w}$ \\
\hline 167.9 & taxdis & 22.0 & $\mathrm{w}$ \\
\hline 172.3 & taxdis & 18.3 & $\mathrm{w}$ \\
\hline 173.1 & \multicolumn{3}{|c|}{ marker \# 132} \\
\hline 173.3 & taxdis & 12.3 & w \\
\hline \multirow{2}{*}{175.5} & \multirow{2}{*}{ frapro } & 65.5 & \multirow{2}{*}{$\mathrm{w}$} \\
\hline & & 37.8 & \\
\hline 176.7 & nysbif & 17.7 & $\mathrm{w}$ \\
\hline 176.9 & nysbif & 13.6 & $\mathrm{w}$ \\
\hline 181.5 & frapro & 23.9 & $\mathrm{w}$ \\
\hline 181.8 & snag & 30.5 & $\mathrm{w}$ \\
\hline 183.0 & nysbif & 61.0 & $\mathrm{w}$ \\
\hline 183.2 & quelau & 19.0 & $\mathrm{w}$ \\
\hline 185.4 & nysbif & 52.4 & $\mathrm{w}$ \\
\hline 190.2 & frapro & 11.5 & $\mathrm{w}$ \\
\hline 190.6 & taxdis & 36.4 & $\mathrm{w}$ \\
\hline 192.8 & taxdis & 21.9 & $\mathrm{w}$ \\
\hline 193.9 & taxdis & 44.0 & $\mathrm{w}$ \\
\hline 196.4 & nysbif & 11.5 & $\mathrm{w}$ \\
\hline 197.3 & nysbif & 25.0 & $\mathrm{w}$ \\
\hline 202.7 & nysaqu & 65.0 & $\mathrm{w}$ \\
\hline 202.9 & nysbif & 10.4 & $\mathrm{w}$ \\
\hline 205.9 & \multicolumn{3}{|c|}{ marker \# 133} \\
\hline 206.8 & taxdis & 14.6 & $\mathrm{w}$ \\
\hline 208.5 & nysaqu & 46.6 & $\mathrm{w}$ \\
\hline 208.9 & nysbif & 17.0 & $\mathrm{w}$ \\
\hline 211.6 & snag & 28.6 & $\mathrm{w}$ \\
\hline 212.5 & taxdis & 53.6 & $\mathrm{w}$ \\
\hline 216.0 & ulmame & 13.1 & $\mathrm{w}$ \\
\hline 216.3 & ulmame & 14.5 & $\mathrm{w}$ \\
\hline 219.9 & frapro & 12.0 & $\mathrm{w}$ \\
\hline 221.5 & nysbif & 49.5 & $\mathrm{w}$ \\
\hline 221.8 & snag & 25.6 & $\mathrm{w}$ \\
\hline 224.2 & taxdis & 24.9 & $\mathrm{w}$ \\
\hline 227.3 & frapro & 20.6 & $\mathrm{w}$ \\
\hline 229.6 & taxdis & 46.9 & $\mathrm{w}$ \\
\hline 231.2 & nysbif & 19.5 & $\mathrm{w}$ \\
\hline
\end{tabular}

\begin{tabular}{|c|c|c|c|}
\hline $\begin{array}{l}\text { Distance from } \\
\text { beginning of } \\
\text { transect, in } m\end{array}$ & $\begin{array}{c}\text { Tree } \\
\text { species }\end{array}$ & $\begin{array}{l}\text { Dbh, } \\
\text { in } \mathrm{cm}\end{array}$ & Side \\
\hline 233.3 & taxdis & 15.6 & $\mathrm{w}$ \\
\hline 238.1 & taxdis & 24.8 & $\mathrm{w}$ \\
\hline 239.2 & taxdis & 22.9 & $\mathrm{w}$ \\
\hline 241.4 & \multicolumn{3}{|c|}{ marker \#134 } \\
\hline 242.6 & magvir & 21.4 & $\mathrm{~W}$ \\
\hline 243.2 & taxdis & 26.9 & $\mathrm{w}$ \\
\hline 243.7 & taxdis & 60.5 & $\mathrm{w}$ \\
\hline 248.0 & ulmame & 13.0 & $\mathrm{w}$ \\
\hline 248.6 & frapro & 16.9 & $\mathrm{w}$ \\
\hline 255.5 & ulmame & 20.2 & $\mathrm{w}$ \\
\hline 256.0 & nysbif & 38.9 & $\mathrm{w}$ \\
\hline 256.8 & nysbif & 59.8 & $\mathrm{w}$ \\
\hline 262.1 & taxdis & 16.0 & $\mathrm{w}$ \\
\hline 262.2 & nysbif & 19.7 & $\mathrm{w}$ \\
\hline 262.3 & nysbif & 12.1 & $\mathrm{w}$ \\
\hline 263.8 & snag & 15.4 & $\mathrm{w}$ \\
\hline 271.0 & \multicolumn{3}{|c|}{ marker \#135 } \\
\hline 272.2 & taxdis & 30.0 & $\mathrm{w}$ \\
\hline 272.3 & snag & 31.1 & $\mathrm{w}$ \\
\hline 276.1 & snag & 61.4 & $\mathrm{w}$ \\
\hline \multirow{2}{*}{276.5} & \multirow{2}{*}{ carcar } & 12.7 & \multirow{2}{*}{$\mathrm{w}$} \\
\hline & & 8.7 & \\
\hline 276.9 & magvir & 41.4 & $\mathrm{w}$ \\
\hline 281.2 & snag & 44.0 & $\mathrm{w}$ \\
\hline 286.2 & nysbif & 17.0 & $\mathrm{w}$ \\
\hline 287.2 & taxdis & 10.6 & $\mathrm{w}$ \\
\hline 293.5 & carcar & 10.2 & $\mathrm{w}$ \\
\hline 295.4 & quelau & 21.7 & $\mathrm{w}$ \\
\hline 295.8 & quelau & 30.7 & $\mathrm{w}$ \\
\hline 297.5 & nysbif & 59.9 & $\mathrm{w}$ \\
\hline 297.6 & liqsty & 22.5 & $\mathrm{w}$ \\
\hline 300.1 & snag & 10.5 & $\mathrm{~W}$ \\
\hline 302.0 & nysbif & 23.0 & $\mathrm{w}$ \\
\hline 305.4 & magvir & 23.4 & $\mathrm{w}$ \\
\hline 305.8 & \multicolumn{3}{|c|}{ marker \# 136} \\
\hline 306.1 & magvir & 52.8 & $\mathrm{w}$ \\
\hline 306.4 & nysbif & 19.1 & $\mathrm{w}$ \\
\hline 313.3 & frapro & 23.2 & $\mathrm{w}$ \\
\hline 313.4 & snag & 34.8 & $\mathrm{w}$ \\
\hline 318.4 & magvir & 55.0 & $\mathrm{w}$ \\
\hline 320.0 & liqsty & 12.2 & $\mathrm{w}$ \\
\hline 322.1 & nysbif & 19.4 & $\mathrm{w}$ \\
\hline 323.6 & nysbif & 11.0 & $\mathrm{w}$ \\
\hline 323.9 & nysbif & 40.6 & $\mathrm{w}$ \\
\hline 325.4 & nysbif & 28.9 & $\mathrm{w}$ \\
\hline 327.8 & magvir & 10.2 & W \\
\hline
\end{tabular}


G TK: The sampling width of this transect is 5 meters on the west side of the transect line

\begin{tabular}{|c|c|c|c|c|c|c|c|}
\hline $\begin{array}{l}\text { Distance from } \\
\text { beginning of } \\
\text { transect, in } m\end{array}$ & $\begin{array}{c}\text { Tree } \\
\text { species }\end{array}$ & $\begin{array}{l}\text { Dbh, } \\
\text { in } \mathrm{cm}\end{array}$ & Side & $\begin{array}{l}\text { Distance from } \\
\text { beginning of } \\
\text { transect, in } \mathrm{m}\end{array}$ & $\begin{array}{c}\text { Tree } \\
\text { species }\end{array}$ & $\begin{array}{l}\text { Dbh, } \\
\text { in } \mathrm{cm}\end{array}$ & Side \\
\hline 328.0 & magvir & 51.3 & $\mathrm{w}$ & 398.5 & frapro & 57.6 & $\mathrm{w}$ \\
\hline 329.5 & liqsty & 11.1 & $\mathrm{w}$ & 399.6 & nysbif & 21.4 & $\mathrm{w}$ \\
\hline 333.0 & \multicolumn{3}{|c|}{ marker \#137 } & 401.6 & frapro & 15.1 & $\mathrm{w}$ \\
\hline 334.4 & nysbif & 34.4 & $\mathrm{w}$ & 403.7 & nysbif & 25.8 & $\mathrm{w}$ \\
\hline 335.4 & nysbif & 17.1 & w & 404.0 & nysbif & 16.6 & $\mathrm{w}$ \\
\hline 336.9 & snag & 24.4 & $\mathrm{w}$ & 404.4 & taxdis & 28.8 & $\mathrm{w}$ \\
\hline 338.1 & nysbif & 23.5 & $\mathrm{w}$ & 405.8 & nysbif & 41.4 & $\mathrm{w}$ \\
\hline 338.3 & taxdis & 13.5 & $\mathrm{w}$ & 406.8 & taxdis & 11.5 & $\mathrm{w}$ \\
\hline 338.4 & nysbif & 17.5 & $\mathrm{w}$ & 411.9 & & \# 140 & \\
\hline 342.7 & liqsty & 26.1 & $\mathrm{w}$ & & & & \\
\hline 343.7 & magvir & 43.6 & $\mathrm{w}$ & & & & \\
\hline 347.1 & taxdis & 42.5 & $\mathrm{w}$ & & & & \\
\hline 349.3 & acerub & 42.9 & $\mathrm{w}$ & & & & \\
\hline 351.2 & nysbif & 34.8 & $\mathrm{w}$ & & & & \\
\hline 352.5 & nysbif & 13.0 & $\mathrm{w}$ & & & & \\
\hline \multirow{2}{*}{356.4} & \multirow{2}{*}{ magvir } & 41.5 & \multirow{2}{*}{$\mathrm{w}$} & & & & \\
\hline & & 6.1 & & & & & \\
\hline 358.1 & nysbif & 29.4 & w & & & & \\
\hline 358.3 & nysbif & 19.0 & $\mathrm{w}$ & & & & \\
\hline 361.1 & \multicolumn{3}{|c|}{ marker \# 138} & & & & \\
\hline 361.4 & nysbif & 21.8 & $\mathrm{w}$ & & & & \\
\hline 362.0 & liqsty & 20.6 & w & & & & \\
\hline 364.1 & frapro & 59.8 & $\mathrm{w}$ & & & & \\
\hline 365.1 & nysbif & 34.3 & $\mathrm{w}$ & & & & \\
\hline 369.7 & nysbif & 11.5 & $\mathrm{w}$ & & & & \\
\hline 371.5 & taxdis & 14.3 & $\mathrm{w}$ & & & & \\
\hline 372.5 & frapro & 50.9 & w & & & & \\
\hline 373.2 & snag & 18.9 & $\mathrm{w}$ & & & & \\
\hline 374.7 & nysbif & 25.3 & $\mathrm{w}$ & & & & \\
\hline 375.8 & nysbif & 19.0 & $\mathrm{w}$ & & & & \\
\hline 376.9 & frapro & 32.4 & $\mathrm{w}$ & & & & \\
\hline 377.4 & snag & 19.5 & $\mathrm{w}$ & & & & \\
\hline 378.5 & ulmame & 10.7 & $\mathrm{w}$ & & & & \\
\hline 380.0 & taxdis & 30.0 & w & & & & \\
\hline 380.6 & quelau & 21.0 & w & & & & \\
\hline 382.8 & taxdis & 41.3 & w & & & & \\
\hline \multirow{2}{*}{384.9} & \multirow{2}{*}{ frapro } & 27.0 & \multirow{2}{*}{$\mathrm{w}$} & & & & \\
\hline & & 12.1 & & & & & \\
\hline 384.9 & frapro & 4.5 & w & & & & \\
\hline 388.4 & nysbif & 38.5 & $\mathrm{w}$ & & & & \\
\hline 390.5 & frapro & 34.2 & w & & & & \\
\hline 390.7 & nysbif & 46.0 & w & & & & \\
\hline 392.2 & \multicolumn{3}{|c|}{ marker \# 139} & & & & \\
\hline 394.1 & nysbif & 11.9 & w & & & & \\
\hline 396.7 & nysbif & 17.5 & w & & & & \\
\hline 396.8 & nysbif & 59.2 & $\mathrm{w}$ & & & & \\
\hline
\end{tabular}


H SN: The sampling width of this transect is 8 meters on the north side of the transect line and 5 meters on the south side of the transect line

\begin{tabular}{|c|c|c|c|c|c|c|c|}
\hline $\begin{array}{l}\text { Distance from } \\
\text { beginning of } \\
\text { transect, in } m\end{array}$ & $\begin{array}{c}\text { Tree } \\
\text { species }\end{array}$ & $\begin{array}{l}\text { Dbh, } \\
\text { in } \mathrm{cm}\end{array}$ & Side & $\begin{array}{l}\text { Distance from } \\
\text { beginning of } \\
\text { transect, in } m\end{array}$ & $\begin{array}{c}\text { Tree } \\
\text { species }\end{array}$ & $\begin{array}{l}\text { Dbh, } \\
\text { in } \mathrm{cm}\end{array}$ & Side \\
\hline 0.0 & \multicolumn{3}{|c|}{ marker \# 141} & 32.8 & magvir & 37.6 & $\mathrm{~s}$ \\
\hline 1.4 & magvir & 21.4 & $\mathrm{~s}$ & 33.1 & sabpal & 31.0 & $\mathrm{n}$ \\
\hline 2.9 & sabpal & 24.2 & $\mathrm{n}$ & 33.5 & snag & 13.5 & $\mathrm{n}$ \\
\hline 3.3 & magvir & 16.0 & $\mathrm{n}$ & 33.5 & snag & 13.0 & $\mathrm{n}$ \\
\hline 3.8 & sabpal & 31.4 & $\mathrm{~s}$ & 33.6 & magvir & 50.3 & $\mathrm{n}$ \\
\hline 5.0 & magvir & 11.9 & $\mathrm{~s}$ & 36.1 & sabpal & 35.6 & $\mathrm{n}$ \\
\hline 6.0 & frapro & 10.3 & $\mathrm{n}$ & 36.1 & nysbif & 25.4 & $\mathrm{n}$ \\
\hline \multirow{3}{*}{6.6} & \multirow{3}{*}{ magvir } & 10.1 & \multirow{3}{*}{$\mathrm{n}$} & 37.5 & magvir & 27.4 & $\mathrm{~s}$ \\
\hline & & 13.0 & & 38.2 & nysbif & 29.9 & $\mathrm{~s}$ \\
\hline & & 30.8 & & 38.3 & perpal & 10.3 & $\mathrm{~s}$ \\
\hline 7.6 & magvir & 14.4 & $\mathrm{n}$ & 38.4 & sabpal & 25.2 & $\mathrm{~s}$ \\
\hline 10.4 & perpal & 15.6 & $\mathrm{~s}$ & 38.6 & \multicolumn{3}{|c|}{ marker \# 143} \\
\hline 10.4 & perpal & 22.6 & $\mathrm{~s}$ & 40.6 & frapro & 10.3 & $\mathrm{~s}$ \\
\hline 10.9 & quenig & 12.7 & $\mathrm{~s}$ & 40.7 & nysbif & 27.6 & $\mathrm{~s}$ \\
\hline 11.2 & frapro & 43.3 & $\mathrm{n}$ & 41.4 & magvir & 34.7 & $\mathrm{n}$ \\
\hline 11.5 & magvir & 10.0 & $\mathrm{n}$ & 41.6 & taxdis & 12.4 & $\mathrm{n}$ \\
\hline 12.8 & nysbif & 18.4 & $\mathrm{n}$ & 42.1 & sabpal & 22.4 & $\mathrm{n}$ \\
\hline 13.3 & nysbif & 26.3 & $\mathrm{n}$ & 43.6 & snag & 25.2 & $\mathrm{~s}$ \\
\hline 14.1 & sabpal & 23.3 & $\mathrm{n}$ & 44.2 & nysbif & 26.6 & $\mathrm{n}$ \\
\hline 14.8 & myrcer & 11.4 & $\mathrm{n}$ & 45.2 & frapro & 33.7 & $\mathrm{n}$ \\
\hline 15.3 & \multicolumn{3}{|c|}{ marker \# 142} & 47.0 & sabpal & 24.9 & $\mathrm{~s}$ \\
\hline 16.2 & sabpal & 24.9 & $\mathrm{~s}$ & 47.2 & sabpal & 24.6 & $\mathrm{n}$ \\
\hline 16.3 & frapro & 40.5 & $\mathrm{n}$ & 47.5 & perpal & 41.3 & $\mathrm{n}$ \\
\hline 17.5 & sabpal & 25.2 & $\mathrm{n}$ & 49.1 & sabpal & 23.3 & $\mathrm{~s}$ \\
\hline 18.3 & sabpal & 22.5 & $\mathrm{~s}$ & 49.2 & nysbif & 10.5 & $\mathrm{n}$ \\
\hline 19.3 & quelau & 50.4 & $\mathrm{~s}$ & 51.4 & sabpal & 16.0 & $\mathrm{n}$ \\
\hline 19.7 & magvir & 36.7 & $\mathrm{n}$ & 52.8 & sabpal & 28.9 & $\mathrm{~s}$ \\
\hline 19.7 & taxdis & 18.6 & $\mathrm{n}$ & 54.1 & sabpal & 23.7 & $\mathrm{n}$ \\
\hline 20.9 & magvir & 38.0 & $\mathrm{~s}$ & 56.1 & taxdis & 14.7 & $\mathrm{n}$ \\
\hline 22.2 & taxdis & 30.5 & $\mathrm{n}$ & 58.1 & magvir & 21.1 & $\mathrm{n}$ \\
\hline 22.9 & frapro & 43.4 & $\mathrm{~s}$ & 58.2 & magvir & 27.2 & $\mathrm{n}$ \\
\hline 23.6 & sabpal & 20.5 & $\mathrm{~s}$ & 59.2 & snag & 22.8 & $\mathrm{~s}$ \\
\hline 24.2 & myrcer & 11.5 & $\mathrm{~s}$ & 59.7 & myrcer & 16.8 & $\mathrm{~s}$ \\
\hline 24.5 & perpal & 19.6 & $\mathrm{~s}$ & 59.8 & nysbif & 41.2 & $\mathrm{~s}$ \\
\hline 26.4 & sabpal & 24.9 & $\mathrm{n}$ & 61.0 & magvir & 34.1 & $\mathrm{n}$ \\
\hline \multirow{2}{*}{27.2} & \multirow{2}{*}{ magvir } & 38.0 & \multirow{2}{*}{$\mathrm{s}$} & 61.0 & magvir & 32.3 & $\mathrm{~s}$ \\
\hline & & 4.5 & & 61.1 & magvir & 19.8 & $\mathrm{~s}$ \\
\hline 27.6 & taxdis & 12.9 & $\mathrm{n}$ & 61.6 & sabpal & 24.3 & $\mathrm{n}$ \\
\hline 29.2 & myrcer & 20.7 & $\mathrm{n}$ & 62.3 & nysbif & 26.3 & $\mathrm{n}$ \\
\hline 29.5 & frapro & 42.2 & $\mathrm{~s}$ & 62.7 & \multicolumn{3}{|c|}{ marker \# 144} \\
\hline 30.0 & perpal & 17.4 & $\mathrm{n}$ & 62.9 & taxdis & 10.3 & $\mathrm{n}$ \\
\hline 30.8 & sabpal & 25.0 & $\mathrm{~s}$ & 63.4 & magvir & 23.4 & $\mathrm{~s}$ \\
\hline 31.1 & sabpal & 33.9 & $\mathrm{~s}$ & 63.5 & magvir & 29.3 & $\mathrm{n}$ \\
\hline 31.6 & sabpal & 24.7 & $\mathrm{~s}$ & 65.1 & myrcer & 14.9 & $\mathrm{n}$ \\
\hline 31.8 & magvir & 20.6 & $\mathrm{~s}$ & 65.1 & magvir & 27.2 & $\mathrm{~s}$ \\
\hline
\end{tabular}


H SN: The sampling width of this transect is 8 meters on the north side of the transect line and 5 meters on the south side of the transect line

\begin{tabular}{|c|c|c|c|}
\hline $\begin{array}{c}\text { Distance from beginning of } \\
\text { transect, in } \mathrm{m}\end{array}$ & $\begin{array}{c}\text { Tree } \\
\text { species }\end{array}$ & $\begin{array}{l}\mathrm{Dbh}, \\
\text { in } \mathrm{cm}\end{array}$ & Side \\
\hline 65.3 & myrcer & 10.4 & $\mathrm{~s}$ \\
\hline 65.4 & ulmame & 16.3 & $\mathrm{n}$ \\
\hline 66.2 & magvir & 25.2 & $\mathrm{n}$ \\
\hline 66.4 & nysbif & 43.1 & $\mathrm{~s}$ \\
\hline 66.8 & frapro & 25.6 & $\mathrm{n}$ \\
\hline 67.8 & magvir & 17.1 & $\mathrm{n}$ \\
\hline 68.1 & magvir & 30.2 & $\mathrm{n}$ \\
\hline 68.3 & frapro & 29.6 & $\mathrm{~s}$ \\
\hline 69.2 & nysbif & 11.6 & $\mathrm{n}$ \\
\hline 70.6 & perpal & 14.5 & $\mathrm{n}$ \\
\hline 71.2 & nysbif & 28.6 & $\mathrm{n}$ \\
\hline 71.7 & sabpal & 22.1 & $\mathrm{n}$ \\
\hline 72.0 & taxdis & 20.2 & $\mathrm{n}$ \\
\hline 72.4 & magvir & 16.2 & $\mathrm{~s}$ \\
\hline 73.4 & taxdis & 28.8 & $\mathrm{n}$ \\
\hline 74.3 & frapro & 29.5 & $\mathrm{n}$ \\
\hline \multirow{2}{*}{74.3} & \multirow{2}{*}{ frapro } & 29.4 & \multirow{2}{*}{$\mathrm{s}$} \\
\hline & & 15.9 & \\
\hline 76.0 & perpal & 12.5 & $\mathrm{~s}$ \\
\hline 78.6 & snag & 40.0 & $\mathrm{~s}$ \\
\hline 79.3 & nysbif & 27.5 & $\mathrm{n}$ \\
\hline \multirow{2}{*}{79.3} & \multirow{2}{*}{ myrcer } & 22.2 & \multirow{2}{*}{$\mathrm{n}$} \\
\hline & & 9.3 & \\
\hline 79.7 & magvir & 23.7 & $\mathrm{~s}$ \\
\hline 80.0 & magvir & 32.3 & $\mathrm{n}$ \\
\hline 80.6 & acerub & 40.0 & $\mathrm{n}$ \\
\hline 82.3 & sabpal & 29.3 & $\mathrm{n}$ \\
\hline 82.5 & taxdis & 40.8 & $\mathrm{~s}$ \\
\hline 82.6 & sabpal & 26.0 & $\mathrm{n}$ \\
\hline 83.5 & taxdis & 24.5 & $\mathrm{~s}$ \\
\hline 84.2 & myrcer & 11.1 & $\mathrm{n}$ \\
\hline 85.3 & snag & 19.5 & $\mathrm{n}$ \\
\hline 86.5 & magvir & 20.6 & $\mathrm{~s}$ \\
\hline 86.7 & magvir & 28.7 & $\mathrm{n}$ \\
\hline 88.3 & sabpal & 23.8 & $\mathrm{~s}$ \\
\hline 88.3 & \multicolumn{3}{|c|}{ marker \# 145} \\
\hline \multirow{2}{*}{88.4} & \multirow{2}{*}{ myrcer } & 11.0 & \multirow{2}{*}{$\mathrm{s}$} \\
\hline & & 8.3 & \\
\hline
\end{tabular}


I SH: The sampling width of this transect is 10 meters, 5 meters on either side of the transect line

\begin{tabular}{|c|c|c|c|c|c|c|c|}
\hline $\begin{array}{l}\text { Distance from } \\
\text { beginning of } \\
\text { transect, in } \mathrm{m}\end{array}$ & $\begin{array}{c}\text { Tree } \\
\text { species }\end{array}$ & $\begin{array}{l}\text { Dbh, } \\
\text { in } \mathrm{cm}\end{array}$ & Side & $\begin{array}{l}\text { Distance from } \\
\text { beginning of } \\
\text { transect, in } \mathrm{m}\end{array}$ & $\begin{array}{c}\text { Tree } \\
\text { species }\end{array}$ & $\begin{array}{l}\text { Dbh, } \\
\text { in } \mathrm{cm}\end{array}$ & Side \\
\hline 0.0 & \multicolumn{3}{|c|}{ marker \# 146} & 73.7 & quelau & 13.7 & $\mathrm{w}$ \\
\hline 0.2 & ulmame & 13.8 & $\mathrm{w}$ & 75.7 & sabpal & 27.3 & $\mathrm{w}$ \\
\hline 0.6 & taxdis & 41.6 & $\mathrm{w}$ & 77.0 & quenig & 15.3 & $\mathrm{e}$ \\
\hline 0.8 & magvir & 34.5 & $\mathrm{e}$ & \multirow{2}{*}{78.4} & \multirow{2}{*}{ quelau } & 11.4 & \multirow{2}{*}{$\mathrm{e}$} \\
\hline 1.6 & sabpal & 31.3 & $\mathrm{e}$ & & & 7.0 & \\
\hline 2.1 & snag & 24.5 & $\mathrm{w}$ & 80.0 & quelau & 11.7 & $\mathrm{e}$ \\
\hline 4.7 & acerub & 16.1 & $\mathrm{w}$ & 83.4 & quelau & 18.0 & $\mathrm{e}$ \\
\hline 6.9 & sabpal & 27.8 & $\mathrm{w}$ & 84.1 & acerub & 10.6 & $\mathrm{e}$ \\
\hline 9.0 & perpal & 20.4 & $\mathrm{w}$ & 84.6 & quelau & 16.8 & w \\
\hline 10.6 & carcar & 10.0 & $\mathrm{w}$ & 84.7 & sabpal & 27.9 & $\mathrm{e}$ \\
\hline 15.7 & carcar & 17.5 & $\mathrm{w}$ & 85.2 & sabpal & 26.8 & $\mathrm{w}$ \\
\hline 16.8 & sabpal & 27.0 & $\mathrm{w}$ & 86.0 & \multicolumn{3}{|c|}{ marker \# 149} \\
\hline 17.6 & sabpal & 34.8 & $\mathrm{~W}$ & 88.4 & sabpal & 26.3 & $\mathrm{e}$ \\
\hline 19.3 & pintae & 28.5 & w & 88.5 & sabpal & 23.1 & $\mathrm{e}$ \\
\hline 25.4 & liqsty & 33.9 & $\mathrm{e}$ & 89.0 & quelau & 29.0 & $\mathrm{e}$ \\
\hline 28.6 & \multicolumn{3}{|c|}{ marker \# 147} & 92.3 & quelau & 15.0 & $\mathrm{e}$ \\
\hline 29.2 & snag & 17.1 & $\mathrm{~W}$ & 93.1 & quelau & 24.5 & $\mathrm{e}$ \\
\hline 31.1 & pintae & 31.7 & $\mathrm{w}$ & 93.8 & junsil & 20.5 & $\mathrm{e}$ \\
\hline 31.2 & quenig & 28.2 & $\mathrm{e}$ & 95.8 & frapro & 19.6 & $\mathrm{e}$ \\
\hline 33.4 & sabpal & 24.5 & $\mathrm{w}$ & 95.9 & ulmame & 18.5 & $\mathrm{e}$ \\
\hline 33.6 & quenig & 21.0 & w & 97.4 & sabpal & 13.2 & $\mathrm{w}$ \\
\hline 35.9 & quenig & 24.5 & w & 97.4 & quelau & 13.3 & w \\
\hline 36.4 & quenig & 31.1 & w & 99.4 & sabpal & 30.0 & $\mathrm{e}$ \\
\hline 36.6 & quenig & 13.8 & w & 99.4 & sabpal & 30.4 & $\mathrm{e}$ \\
\hline 38.3 & quenig & 32.1 & w & 100.8 & sabpal & 26.9 & $\mathrm{e}$ \\
\hline 41.5 & sabpal & 23.6 & $\mathrm{~W}$ & 105.2 & quelau & 33.0 & $\mathrm{e}$ \\
\hline 41.5 & pintae & 14.4 & $\mathrm{w}$ & 105.3 & sabpal & 25.5 & w \\
\hline 43.2 & sabpal & 22.2 & $\mathrm{e}$ & 106.2 & sabpal & 31.6 & $\mathrm{e}$ \\
\hline 43.2 & quenig & 33.3 & $\mathrm{w}$ & 106.5 & liqsty & 28.7 & $\mathrm{w}$ \\
\hline 43.9 & quevir & 41.5 & $\mathrm{e}$ & 107.0 & liqsty & 32.5 & $\mathrm{e}$ \\
\hline 44.5 & pintae & 17.3 & $\mathrm{e}$ & 107.3 & sabpal & 22.1 & $\mathrm{w}$ \\
\hline 49.2 & sabpal & 31.9 & $\mathrm{e}$ & 107.8 & sabpal & 29.1 & $\mathrm{w}$ \\
\hline 50.0 & \multicolumn{3}{|c|}{ marker \# 148} & 108.2 & sabpal & 32.3 & $\mathrm{w}$ \\
\hline 53.3 & liqsty & 14.6 & $\mathrm{w}$ & 109.9 & quevir & 19.1 & w \\
\hline 53.6 & sabpal & 30.1 & w & 111.2 & pintae & 27.8 & $\mathrm{e}$ \\
\hline 58.5 & sabpal & 26.5 & $\mathrm{w}$ & 111.6 & sabpal & 30.7 & $\mathrm{w}$ \\
\hline 58.8 & acerub & 12.8 & w & 113.3 & sabpal & 17.9 & $\mathrm{w}$ \\
\hline 59.1 & sabpal & 28.2 & $\mathrm{~W}$ & 114.1 & sabpal & 27.4 & $\mathrm{e}$ \\
\hline 59.3 & pintae & 52.5 & $\mathrm{e}$ & 116.1 & sabpal & 27.2 & $\mathrm{e}$ \\
\hline 62.0 & quenig & 16.9 & $\mathrm{e}$ & 117.4 & \multicolumn{3}{|c|}{ marker \# 150} \\
\hline 62.6 & sabpal & 21.4 & $\mathrm{e}$ & 118.8 & liqsty & 17.1 & $\mathrm{e}$ \\
\hline 64.0 & ostvir & 14.0 & $\mathrm{w}$ & 119.4 & sabpal & 13.3 & $\mathrm{w}$ \\
\hline 65.9 & quelau & 17.7 & $\mathrm{w}$ & 120.2 & quelau & 18.8 & $\mathrm{w}$ \\
\hline 67.4 & sabpal & 27.4 & $\mathrm{w}$ & 121.5 & junsil & 14.9 & $\mathrm{e}$ \\
\hline 69.3 & sabpal & 30.0 & $\mathrm{e}$ & 124.3 & junsil & 25.8 & $\mathrm{w}$ \\
\hline 73.7 & sabpal & 27.0 & $\mathrm{w}$ & 124.3 & vitcin & 11.6 & $\mathrm{w}$ \\
\hline
\end{tabular}


I SH: The sampling width of this transect is 10 meters, 5 meters on either side of the transect line (Continued)

\begin{tabular}{|c|c|c|c|}
\hline $\begin{array}{l}\text { Distance from } \\
\text { beginning of } \\
\text { transect, in } \mathrm{m}\end{array}$ & $\begin{array}{c}\text { Tree } \\
\text { species }\end{array}$ & $\begin{array}{l}\text { Dbh, } \\
\text { in cm }\end{array}$ & Side \\
\hline 124.8 & quevir & 26.0 & $\mathrm{w}$ \\
\hline 125.7 & quevir & 54.9 & $\mathrm{w}$ \\
\hline 128.0 & sabpal & 18.4 & e \\
\hline 128.7 & sabpal & 29.9 & $\mathrm{w}$ \\
\hline 130.9 & sabpal & 25.6 & w \\
\hline 131.2 & sabpal & 26.2 & $\mathrm{e}$ \\
\hline 132.8 & sabpal & 25.0 & $\mathrm{w}$ \\
\hline 134.7 & carcar & 13.4 & $\mathrm{e}$ \\
\hline 136.0 & sabpal & 29.9 & $\mathrm{e}$ \\
\hline 136.5 & sabpal & 24.2 & w \\
\hline 140.6 & sabpal & 28.1 & $\mathrm{e}$ \\
\hline 141.7 & sabpal & 28.0 & $\mathrm{w}$ \\
\hline 141.9 & sabpal & 26.3 & $\mathrm{e}$ \\
\hline 144.0 & sabpal & 25.8 & $\mathrm{w}$ \\
\hline 144.0 & sabpal & 23.9 & $\mathrm{e}$ \\
\hline 147.2 & sabpal & 22.3 & $\mathrm{e}$ \\
\hline 148.9 & sabpal & 30.5 & $\mathrm{e}$ \\
\hline 151.0 & \multicolumn{3}{|c|}{ marker \# 151} \\
\hline 155.0 & quevir & 62.2 & $\mathrm{e}$ \\
\hline
\end{tabular}


J BC: The sampling width of this transect is 10 meters, 5 meters on either side of the transect line

\begin{tabular}{|c|c|c|c|}
\hline $\begin{array}{l}\text { Distance from } \\
\text { beginning of } \\
\text { transect, in } \mathrm{m}\end{array}$ & $\begin{array}{c}\text { Tree } \\
\text { species }\end{array}$ & $\begin{array}{l}\text { Dbh, } \\
\text { in cm }\end{array}$ & Side \\
\hline 0.0 & \multicolumn{3}{|c|}{ marker \# 152} \\
\hline 3.3 & magvir & 13.6 & $\mathrm{e}$ \\
\hline 3.5 & myrcer & 17.8 & $\mathrm{e}$ \\
\hline 3.7 & quevir & 38.0 & $\mathrm{e}$ \\
\hline 4.2 & sabpal & 29.5 & w \\
\hline 4.6 & acerub & 24.4 & $\mathrm{e}$ \\
\hline 5.3 & taxdis & 16.5 & $\mathrm{w}$ \\
\hline 5.7 & frapro & 34.1 & $\mathrm{w}$ \\
\hline 8.0 & nysbif & 35.0 & w \\
\hline 9.0 & acerub & 21.4 & w \\
\hline \multirow{2}{*}{10.0} & \multirow{2}{*}{ ulmame } & 19.9 & \multirow{2}{*}{$\mathrm{e}$} \\
\hline & & 21.1 & \\
\hline 10.5 & sabpal & 22.6 & $\mathrm{w}$ \\
\hline 10.7 & quelau & 10.2 & $\mathrm{~W}$ \\
\hline 12.3 & sabpal & 28.2 & $\mathrm{e}$ \\
\hline 12.7 & frapro & 14.0 & W \\
\hline 12.8 & taxdis & 10.5 & $\mathrm{e}$ \\
\hline 14.3 & magvir & 44.8 & $\mathrm{e}$ \\
\hline 14.7 & acerub & 40.2 & $\mathrm{w}$ \\
\hline 15.6 & ulmame & 23.4 & $\mathrm{w}$ \\
\hline 16.6 & magvir & 12.3 & $\mathrm{w}$ \\
\hline 18.5 & ulmame & 32.2 & $\mathrm{e}$ \\
\hline 18.7 & magvir & 22.4 & $\mathrm{e}$ \\
\hline 21.1 & quelau & 14.3 & $\mathrm{w}$ \\
\hline 21.2 & quelau & 12.4 & $\mathrm{e}$ \\
\hline 22.4 & frapro & 17.2 & $\mathrm{e}$ \\
\hline 22.5 & frapro & 15.4 & $\mathrm{w}$ \\
\hline 22.7 & quelau & 14.1 & $\mathrm{e}$ \\
\hline 22.8 & nysbif & 17.4 & $\mathrm{w}$ \\
\hline 23.8 & \multicolumn{3}{|c|}{ marker \# 153} \\
\hline 25.4 & quelau & 13.5 & $\mathrm{e}$ \\
\hline 25.5 & sabpal & 18.4 & $\mathrm{e}$ \\
\hline 26.3 & sabpal & 26.0 & $\mathrm{w}$ \\
\hline 27.3 & nysbif & 19.8 & $\mathrm{e}$ \\
\hline 28.0 & nysbif & 20.0 & w \\
\hline 29.1 & frapro & 43.8 & $\mathrm{e}$ \\
\hline \multirow{5}{*}{29.1} & \multirow{5}{*}{ magvir } & 10.2 & \multirow{5}{*}{ w } \\
\hline & & 17.4 & \\
\hline & & 8.9 & \\
\hline & & 32.0 & \\
\hline & & 12.3 & \\
\hline 29.5 & frapro & 18.0 & w \\
\hline 29.6 & quelau & 10.5 & $\mathrm{w}$ \\
\hline 31.8 & acerub & 51.9 & $\mathrm{w}$ \\
\hline 32.0 & ulmame & 23.4 & $\mathrm{w}$ \\
\hline 32.8 & frapro & 33.1 & $\mathrm{e}$ \\
\hline
\end{tabular}

\begin{tabular}{|c|c|c|c|}
\hline $\begin{array}{l}\text { Distance from } \\
\text { beginning of } \\
\text { transect, in } \mathrm{m}\end{array}$ & $\begin{array}{c}\text { Tree } \\
\text { species }\end{array}$ & $\begin{array}{l}\text { Dbh, } \\
\text { in cm }\end{array}$ & Side \\
\hline 33.0 & frapro & 17.9 & $\mathrm{~W}$ \\
\hline 33.2 & frapro & 21.8 & w \\
\hline 33.2 & sabpal & 24.0 & $\mathrm{e}$ \\
\hline \multirow{4}{*}{35.1} & \multirow{4}{*}{ magvir } & 19.0 & \multirow{4}{*}{ e } \\
\hline & & 12.3 & \\
\hline & & 16.0 & \\
\hline & & 35.6 & \\
\hline 36.8 & frapro & 47.5 & $\mathrm{w}$ \\
\hline 36.8 & taxdis & 12.4 & $\mathrm{e}$ \\
\hline 38.7 & sabpal & 24.0 & $\mathrm{w}$ \\
\hline 40.5 & frapro & 26.5 & $\mathrm{e}$ \\
\hline 41.6 & frapro & 26.4 & $\mathrm{e}$ \\
\hline 42.6 & frapro & 16.9 & $\mathrm{e}$ \\
\hline 43.2 & frapro & 26.3 & $\mathrm{w}$ \\
\hline 43.4 & frapro & 26.2 & $\mathrm{e}$ \\
\hline 43.5 & frapro & 24.5 & $\mathrm{w}$ \\
\hline 43.7 & frapro & 21.4 & $\mathrm{e}$ \\
\hline 43.7 & frapro & 21.1 & $\mathrm{w}$ \\
\hline 44.4 & quelau & 29.5 & $\mathrm{w}$ \\
\hline 44.8 & \multicolumn{3}{|c|}{ marker \# 154} \\
\hline 45.8 & frapro & 24.4 & w \\
\hline 45.9 & frapro & 24.7 & $\mathrm{w}$ \\
\hline 47.1 & frapro & 21.4 & $\mathrm{e}$ \\
\hline 47.9 & taxdis & 11.2 & $\mathrm{e}$ \\
\hline 48.1 & frapro & 21.7 & $\mathrm{w}$ \\
\hline 48.5 & frapro & 16.0 & $\mathrm{e}$ \\
\hline 48.7 & frapro & 27.4 & $\mathrm{w}$ \\
\hline 49.0 & frapro & 38.6 & $\mathrm{e}$ \\
\hline 50.4 & snag & 11.0 & $\mathrm{w}$ \\
\hline 52.7 & sabpal & 20.7 & $\mathrm{e}$ \\
\hline \multirow{2}{*}{53.2} & \multirow{2}{*}{ frapro } & 13.4 & \multirow{2}{*}{$\mathrm{e}$} \\
\hline & & 17.7 & \\
\hline 53.6 & nysbif & 27.5 & $\mathrm{e}$ \\
\hline 55.4 & junsil & 10.4 & w \\
\hline 55.6 & magvir & 33.9 & $\mathrm{w}$ \\
\hline \multirow{2}{*}{55.6} & \multirow{2}{*}{ myrcer } & 11.9 & \multirow{2}{*}{$\mathrm{w}$} \\
\hline & & 6.4 & \\
\hline \multirow{2}{*}{56.6} & \multirow{2}{*}{ frapro } & 26.4 & \multirow{2}{*}{$\mathrm{e}$} \\
\hline & & 27.8 & \\
\hline 56.7 & taxdis & 21.1 & $\mathrm{e}$ \\
\hline 56.8 & frapro & 29.5 & $\mathrm{e}$ \\
\hline 58.1 & frapro & 11.4 & $\mathrm{e}$ \\
\hline 59.2 & frapro & 22.4 & $\mathrm{e}$ \\
\hline 59.5 & frapro & 27.0 & $\mathrm{w}$ \\
\hline 59.9 & frapro & 33.5 & $\mathrm{w}$ \\
\hline 62.5 & frapro & 19.0 & $\mathrm{w}$ \\
\hline
\end{tabular}


J BC: The sampling width of this transect is 10 meters, 5 meters on either side of the transect line (Continued)

\begin{tabular}{|c|c|c|c|c|c|c|c|}
\hline $\begin{array}{l}\text { Distance from } \\
\text { beginning of } \\
\text { transect, in } m\end{array}$ & $\begin{array}{c}\text { Tree } \\
\text { species }\end{array}$ & $\begin{array}{l}\text { Dbh, } \\
\text { in cm }\end{array}$ & Side & $\begin{array}{l}\text { Distance from } \\
\text { beginning of } \\
\text { transect, in } \mathrm{m}\end{array}$ & $\begin{array}{c}\text { Tree } \\
\text { species }\end{array}$ & $\begin{array}{l}\text { Dbh, } \\
\text { in } \mathrm{cm}\end{array}$ & Side \\
\hline 62.6 & frapro & 27.1 & $\mathrm{e}$ & 91.0 & acerub & 10.5 & $\mathrm{w}$ \\
\hline 62.8 & frapro & 28.6 & $\mathrm{e}$ & 92.3 & taxdis & 42.3 & $\mathrm{e}$ \\
\hline 63.6 & frapro & 16.9 & $\mathrm{e}$ & 93.0 & taxdis & 42.2 & $\mathrm{w}$ \\
\hline 65.1 & junsil & 13.1 & $\mathrm{w}$ & 97.0 & frapro & 20.1 & $\mathrm{e}$ \\
\hline 65.4 & sabpal & 23.0 & $\mathrm{w}$ & 97.6 & frapro & 30.2 & $\mathrm{e}$ \\
\hline 65.5 & taxdis & 10.0 & $\mathrm{w}$ & \multirow{2}{*}{100.0} & \multirow{2}{*}{ frapro } & 10.3 & \multirow{2}{*}{ e } \\
\hline \multirow{2}{*}{66.0} & \multirow{2}{*}{ frapro } & 16.7 & \multirow{2}{*}{$\mathrm{e}$} & & & 10.0 & \\
\hline & & 8.4 & & 101.3 & frapro & 26.8 & $\mathrm{e}$ \\
\hline 70.2 & nysbif & 29.7 & $\mathrm{w}$ & 101.7 & frapro & 17.5 & $\mathrm{e}$ \\
\hline 70.6 & frapro & 12.7 & $\mathrm{e}$ & 101.7 & frapro & 40.6 & w \\
\hline 70.7 & frapro & 22.0 & $\mathrm{w}$ & 101.9 & frapro & 31.1 & $\mathrm{e}$ \\
\hline 72.2 & frapro & 28.5 & $\mathrm{w}$ & 102.0 & snag & 11.7 & $\mathrm{e}$ \\
\hline 72.4 & snag & 40.4 & $\mathrm{e}$ & 102.9 & quelau & 12.7 & w \\
\hline 72.6 & frapro & 29.0 & $\mathrm{e}$ & 103.6 & frapro & 25.9 & $\mathrm{e}$ \\
\hline 73.0 & \multicolumn{3}{|c|}{ marker \# 155} & 103.7 & frapro & 27.2 & w \\
\hline 73.4 & frapro & 22.7 & $\mathrm{w}$ & 103.8 & \multicolumn{3}{|c|}{ marker \# 156} \\
\hline 74.3 & frapro & 13.3 & $\mathrm{w}$ & 104.3 & snag & 22.0 & $\mathrm{e}$ \\
\hline \multirow{2}{*}{75.0} & \multirow{2}{*}{ frapro } & 22.0 & \multirow{2}{*}{ e } & 104.9 & nysbif & 36.9 & $\mathrm{e}$ \\
\hline & & 19.6 & & 105.0 & frapro & 14.4 & w \\
\hline 75.5 & frapro & 31.9 & w & 105.5 & frapro & 25.3 & $\mathrm{e}$ \\
\hline 75.6 & frapro & 22.0 & w & 106.3 & quelau & 10.8 & $\mathrm{e}$ \\
\hline 76.4 & frapro & 32.5 & $\mathrm{w}$ & 107.3 & frapro & 17.3 & $\mathrm{e}$ \\
\hline \multirow{3}{*}{77.7} & \multirow{3}{*}{ frapro } & 17.3 & \multirow{3}{*}{$\mathrm{e}$} & 107.3 & frapro & 18.0 & $\mathrm{e}$ \\
\hline & & 13.5 & & 111.0 & acerub & 14.2 & w \\
\hline & & 10.3 & & 113.8 & nysbif & 35.0 & $\mathrm{e}$ \\
\hline 79.9 & nysbif & 30.0 & $\mathrm{w}$ & 114.2 & frapro & 25.4 & $\mathrm{e}$ \\
\hline 80.1 & frapro & 17.4 & $\mathrm{w}$ & 114.6 & taxdis & 29.8 & $\mathrm{e}$ \\
\hline 80.5 & taxdis & 27.7 & $\mathrm{e}$ & 115.0 & frapro & 20.6 & w \\
\hline 80.9 & magvir & 25.7 & $\mathrm{w}$ & 115.9 & junsil & 17.7 & w \\
\hline 81.1 & frapro & 17.7 & $\mathrm{e}$ & 116.3 & frapro & 21.5 & $\mathrm{e}$ \\
\hline 81.1 & frapro & 15.0 & $\mathrm{e}$ & 116.3 & quelau & 11.8 & $\mathrm{e}$ \\
\hline 81.2 & frapro & 14.2 & $\mathrm{e}$ & 118.7 & sabpal & 27.5 & $\mathrm{e}$ \\
\hline 82.1 & snag & 25.4 & $\mathrm{e}$ & 119.3 & taxdis & 21.5 & w \\
\hline 82.7 & frapro & 22.2 & w & 119.4 & frapro & 20.7 & w \\
\hline 82.8 & taxdis & 32.1 & $\mathrm{e}$ & 120.1 & frapro & 24.5 & w \\
\hline 84.9 & frapro & 32.7 & $\mathrm{e}$ & 120.3 & frapro & 17.9 & $\mathrm{e}$ \\
\hline 84.9 & frapro & 24.8 & $\mathrm{e}$ & 124.1 & taxdis & 121.0 & w \\
\hline 85.0 & frapro & 22.2 & w & 124.9 & acerub & 52.0 & $\mathrm{e}$ \\
\hline 85.5 & taxdis & 27.2 & $\mathrm{e}$ & 125.9 & sabpal & 25.6 & $\mathrm{e}$ \\
\hline 85.6 & frapro & 20.0 & w & 127.2 & sabpal & 28.3 & w \\
\hline 86.4 & frapro & 37.3 & $\mathrm{w}$ & 127.6 & sabpal & 26.8 & w \\
\hline 87.0 & frapro & 20.0 & $\mathrm{e}$ & 130.3 & frapro & 33.0 & w \\
\hline 89.2 & frapro & 26.9 & $\mathrm{e}$ & 130.9 & frapro & 22.9 & w \\
\hline 89.8 & frapro & 16.5 & $\mathrm{e}$ & 131.2 & taxdis & 15.2 & $\mathrm{e}$ \\
\hline 90.3 & frapro & 29.4 & $\mathrm{e}$ & 131.8 & taxdis & 22.7 & $\mathrm{e}$ \\
\hline 90.7 & frapro & 27.4 & $\mathrm{e}$ & 132.9 & frapro & 20.2 & $\mathrm{e}$ \\
\hline
\end{tabular}


J BC: The sampling width of this transect is 10 meters, 5 meters on either side of the transect line (Continued)

\begin{tabular}{|c|c|c|c|c|c|c|c|}
\hline $\begin{array}{l}\text { Distance from } \\
\text { beginning of } \\
\text { transect, in } \mathrm{m}\end{array}$ & $\begin{array}{c}\text { Tree } \\
\text { species }\end{array}$ & $\begin{array}{l}\text { Dbh, } \\
\text { in cm }\end{array}$ & Side & $\begin{array}{l}\text { Distance from } \\
\text { beginning of } \\
\text { transect, in } m\end{array}$ & $\begin{array}{c}\text { Tree } \\
\text { species }\end{array}$ & $\begin{array}{l}\text { Dbh, } \\
\text { in } \mathrm{cm}\end{array}$ & Side \\
\hline 134.0 & sabpal & 25.2 & $\mathrm{e}$ & 179.4 & nysbif & 27.8 & $\mathrm{~W}$ \\
\hline 134.9 & nysbif & 13.2 & w & 181.3 & magvir & 15.1 & $\mathrm{e}$ \\
\hline 135.3 & ulmame & 36.5 & $\mathrm{e}$ & 182.0 & taxdis & 31.1 & $\mathrm{e}$ \\
\hline 135.6 & sabpal & 19.9 & w & \multirow{2}{*}{182.6} & \multirow{2}{*}{ frapro } & 21.4 & \multirow{2}{*}{$\mathrm{w}$} \\
\hline 136.2 & acerub & 33.8 & w & & & 12.4 & \\
\hline 137.1 & sabpal & 26.1 & $\mathrm{e}$ & \multirow{2}{*}{183.8} & \multirow{2}{*}{ frapro } & 13.2 & \multirow{2}{*}{$\mathrm{e}$} \\
\hline 139.0 & frapro & 42.4 & w & & & 11.1 & \\
\hline 140.1 & sabpal & 24.8 & $\mathrm{e}$ & 184.0 & frapro & 14.9 & $\mathrm{e}$ \\
\hline 141.5 & acerub & 70.0 & $\mathrm{e}$ & 184.4 & frapro & 24.9 & $\mathrm{w}$ \\
\hline 141.5 & taxdis & 12.4 & $\mathrm{w}$ & \multirow{2}{*}{186.3} & \multirow{2}{*}{ nysbif } & 30.6 & \multirow{2}{*}{$\mathrm{W}$} \\
\hline 143.9 & frapro & 31.7 & $\mathrm{e}$ & & & 24.9 & \\
\hline 144.2 & taxdis & 15.1 & w & 186.8 & carcar & 10.0 & $\mathrm{w}$ \\
\hline 145.9 & frapro & 10.9 & $\mathrm{e}$ & 188.0 & frapro & 13.4 & $\mathrm{e}$ \\
\hline 146.3 & acerub & 42.8 & $\mathrm{w}$ & 188.1 & taxdis & 17.3 & $\mathrm{e}$ \\
\hline 146.6 & frapro & 11.1 & $\mathrm{e}$ & 189.1 & \multicolumn{3}{|c|}{ marker \# 158} \\
\hline 146.9 & myrcer & 12.2 & $\mathrm{e}$ & 189.8 & frapro & 24.8 & $\mathrm{e}$ \\
\hline 147.3 & \multicolumn{3}{|c|}{ marker \# 157} & 191.0 & frapro & 44.1 & $\mathrm{e}$ \\
\hline 150.9 & pintae & 20.6 & $\mathrm{e}$ & 191.0 & frapro & 33.5 & $\mathrm{w}$ \\
\hline 151.3 & ulmame & 10.9 & $\mathrm{e}$ & 193.2 & taxdis & 47.5 & $\mathrm{e}$ \\
\hline 151.9 & junsil & 16.1 & $\mathrm{e}$ & 195.9 & junsil & 10.9 & $\mathrm{e}$ \\
\hline 153.1 & sabpal & 27.9 & $\mathrm{e}$ & 196.2 & nysbif & 10.3 & $\mathrm{e}$ \\
\hline 154.0 & frapro & 67.0 & $\mathrm{e}$ & 196.3 & sabpal & 25.8 & $\mathrm{w}$ \\
\hline 154.5 & taxdis & 11.2 & w & 197.3 & sabpal & 18.3 & $\mathrm{e}$ \\
\hline 155.8 & ulmame & 29.8 & w & 200.2 & acerub & 33.2 & $\mathrm{w}$ \\
\hline 157.7 & frapro & 21.0 & $\mathrm{e}$ & 200.8 & ulmame & 34.7 & $\mathrm{w}$ \\
\hline 159.0 & taxdis & 28.5 & w & 201.2 & magvir & 11.5 & $\mathrm{e}$ \\
\hline 159.8 & taxdis & 46.9 & w & 202.9 & taxdis & 45.2 & $\mathrm{e}$ \\
\hline 160.0 & quelau & 10.2 & $\mathrm{e}$ & 203.7 & frapro & 10.9 & $\mathrm{w}$ \\
\hline 160.2 & taxdis & 26.6 & w & 203.8 & frapro & 38.7 & $\mathrm{e}$ \\
\hline 161.7 & frapro & 23.4 & w & 204.3 & frapro & 14.2 & $\mathrm{w}$ \\
\hline 163.9 & ulmame & 40.2 & $\mathrm{e}$ & 206.9 & frapro & 21.9 & $\mathrm{w}$ \\
\hline 164.2 & taxdis & 15.8 & w & 208.0 & taxdis & 13.6 & $\mathrm{e}$ \\
\hline 164.5 & frapro & 24.0 & $\mathrm{e}$ & 208.1 & ulmame & 25.2 & $\mathrm{e}$ \\
\hline 164.5 & taxdis & 15.0 & w & 208.4 & nysbif & 36.7 & $\mathrm{e}$ \\
\hline 164.7 & acerub & 45.0 & w & 210.6 & nysbif & 20.7 & $\mathrm{w}$ \\
\hline 166.3 & snag & 16.4 & w & 210.8 & frapro & 22.0 & $\mathrm{w}$ \\
\hline 167.1 & taxdis & 33.3 & w & 211.2 & nysbif & 26.7 & $\mathrm{e}$ \\
\hline 167.9 & frapro & 31.8 & $\mathrm{e}$ & 212.2 & snag & 19.4 & $\mathrm{e}$ \\
\hline 168.8 & sabpal & 25.8 & w & 213.4 & nysbif & 24.5 & $\mathrm{w}$ \\
\hline 171.0 & frapro & 28.8 & $\mathrm{e}$ & 213.5 & snag & 14.7 & w \\
\hline 171.0 & taxdis & 27.8 & w & 214.5 & frapro & 24.0 & $\mathrm{e}$ \\
\hline 172.2 & frapro & 42.9 & w & 215.6 & & 159 & \\
\hline 175.0 & taxdis & 38.7 & w & & & & \\
\hline 175.6 & magvir & 43.4 & $\mathrm{e}$ & & & & \\
\hline 178.3 & sabpal & 24.3 & $\mathrm{e}$ & & & & \\
\hline 178.9 & frapro & 22.1 & $\mathrm{e}$ & & & & \\
\hline
\end{tabular}


K LK: The sampling width of this transect is 10 meters, 5 meters on either side of the transect line

\begin{tabular}{|c|c|c|c|c|c|c|c|}
\hline $\begin{array}{l}\text { Distance from } \\
\text { beginning of } \\
\text { transect, in } \mathrm{m}\end{array}$ & $\begin{array}{c}\text { Tree } \\
\text { species }\end{array}$ & Dbh, in $\mathbf{c m}$ & Side & $\begin{array}{l}\text { Distance from } \\
\text { beginning of } \\
\text { transect, in } \mathrm{m}\end{array}$ & $\begin{array}{c}\text { Tree } \\
\text { species }\end{array}$ & $\mathrm{Dbh}$, in $\mathbf{c m}$ & Side \\
\hline 0.0 & \multicolumn{3}{|c|}{ marker \# 160} & 34.1 & magvir & 15.7 & $\mathrm{e}$ \\
\hline \multirow{2}{*}{1.8} & \multirow{2}{*}{ frapro } & 15.0 & \multirow{2}{*}{$\mathrm{w}$} & 36.0 & frapro & 23.7 & $\mathrm{e}$ \\
\hline & & 8.8 & & 36.5 & frapro & 15.3 & $\mathrm{e}$ \\
\hline 1.9 & magvir & 15.5 & $\mathrm{e}$ & 36.8 & frapro & 24.9 & $\mathrm{w}$ \\
\hline 3.2 & frapro & 13.4 & $\mathrm{w}$ & 37.2 & frapro & 19.9 & $\mathrm{w}$ \\
\hline 4.9 & nysbif & 22.2 & $\mathrm{~W}$ & 37.9 & magvir & 12.1 & $\mathrm{e}$ \\
\hline 5.1 & frapro & 13.8 & $\mathrm{w}$ & 38.3 & magvir & 13.3 & $\mathrm{e}$ \\
\hline 6.3 & frapro & 18.0 & $\mathrm{w}$ & 38.7 & snag & 13.9 & $\mathrm{e}$ \\
\hline 9.4 & myrcer & 10.0 & $\mathrm{e}$ & 38.8 & nysbif & 25.4 & $\mathrm{w}$ \\
\hline 9.7 & frapro & 15.7 & $\mathrm{w}$ & 39.0 & nysbif & 11.1 & $\mathrm{e}$ \\
\hline 10.3 & frapro & 14.4 & $\mathrm{w}$ & 39.6 & frapro & 12.8 & $\mathrm{e}$ \\
\hline 11.0 & nysbif & 10.7 & w & \multirow{2}{*}{39.7} & \multirow{2}{*}{ nysbif } & 11.8 & \multirow{2}{*}{$\mathrm{w}$} \\
\hline 13.1 & acerub & 23.5 & $\mathrm{e}$ & & & 6.7 & \\
\hline 13.2 & sabpal & 23.1 & $\mathrm{e}$ & 40.3 & frapro & 21.4 & $\mathrm{w}$ \\
\hline \multirow{2}{*}{13.5} & \multirow{2}{*}{ frapro } & 24.5 & \multirow{2}{*}{$\mathrm{w}$} & 43.1 & magvir & 27.6 & w \\
\hline & & 11.5 & & 43.2 & snag & 13.5 & w \\
\hline 13.7 & nysbif & 11.4 & $\mathrm{e}$ & 44.0 & taxdis & 15.0 & $\mathrm{e}$ \\
\hline 14.6 & magvir & 41.0 & $\mathrm{e}$ & 44.2 & magvir & 14.4 & w \\
\hline 15.3 & myrcer & 10.0 & $\mathrm{e}$ & 44.5 & nysbif & 24.5 & $\mathrm{e}$ \\
\hline 15.6 & nysbif & 15.8 & w & 44.9 & nysbif & 46.3 & W \\
\hline 18.4 & frapro & 10.0 & $\mathrm{w}$ & 45.3 & taxdis & 13.3 & $\mathrm{e}$ \\
\hline 18.5 & sabpal & 28.3 & $\mathrm{e}$ & 45.8 & magvir & 31.6 & w \\
\hline 18.8 & myrcer & 15.5 & w & 46.0 & nysbif & 19.5 & w \\
\hline 19.0 & nysbif & 14.7 & $\mathrm{e}$ & 46.1 & quelau & 14.9 & w \\
\hline 21.2 & frapro & 24.5 & $\mathrm{e}$ & 46.5 & sabpal & 23.0 & w \\
\hline 21.6 & frapro & 21.9 & $\mathrm{w}$ & 48.2 & frapro & 12.6 & w \\
\hline 22.2 & frapro & 10.5 & $\mathrm{w}$ & 48.2 & nysbif & 10.0 & $\mathrm{e}$ \\
\hline 22.3 & magvir & 10.4 & $\mathrm{e}$ & 48.6 & frapro & 10.4 & w \\
\hline 23.2 & magvir & 36.9 & $\mathrm{e}$ & 48.6 & taxdis & 11.2 & $\mathrm{e}$ \\
\hline 23.4 & frapro & 19.0 & w & 49.0 & frapro & 10.0 & $\mathrm{e}$ \\
\hline 23.7 & magvir & 37.4 & w & 50.2 & junsil & 12.3 & $\mathrm{w}$ \\
\hline 24.4 & magvir & 10.4 & $\mathrm{w}$ & 50.3 & frapro & 13.1 & $\mathrm{e}$ \\
\hline 26.0 & frapro & 12.0 & $\mathrm{e}$ & 50.5 & frapro & 10.3 & $\mathrm{e}$ \\
\hline 27.9 & magvir & 22.7 & $\mathrm{e}$ & 52.2 & nysbif & 34.4 & $\mathrm{w}$ \\
\hline 27.9 & magvir & 11.1 & w & 53.0 & magvir & 29.4 & $\mathrm{e}$ \\
\hline 28.2 & frapro & 11.4 & w & \multirow{2}{*}{53.9} & \multirow{2}{*}{ frapro } & 11.0 & \multirow{2}{*}{ w } \\
\hline 28.7 & \multicolumn{3}{|c|}{ marker \# 161} & & & 6.3 & \\
\hline 28.8 & nysbif & 21.4 & $\mathrm{e}$ & 54.2 & frapro & 10.2 & $\mathrm{~W}$ \\
\hline 29.9 & magvir & 36.0 & $\mathrm{w}$ & 54.7 & nysbif & 12.8 & $\mathrm{e}$ \\
\hline 30.8 & frapro & 11.6 & $\mathrm{e}$ & 56.0 & nysbif & 34.0 & $\mathrm{w}$ \\
\hline \multirow{2}{*}{31.4} & \multirow{2}{*}{ frapro } & 10.0 & \multirow{2}{*}{$\mathrm{w}$} & 56.1 & magvir & 17.8 & $\mathrm{e}$ \\
\hline & & 6.0 & & 56.6 & nysbif & 13.0 & $\mathrm{e}$ \\
\hline 32.3 & frapro & 12.9 & $\mathrm{w}$ & 57.3 & frapro & 10.1 & $\mathrm{e}$ \\
\hline 32.4 & frapro & 13.2 & $\mathrm{w}$ & \multirow{2}{*}{58.1} & \multirow{2}{*}{ frapro } & 10.6 & \multirow{2}{*}{$\mathrm{e}$} \\
\hline 33.2 & frapro & 19.1 & $\mathrm{e}$ & & & 7.9 & \\
\hline 33.7 & frapro & 11.4 & $\mathrm{e}$ & 58.2 & frapro & 13.1 & w \\
\hline
\end{tabular}


K LK: The sampling width of this transect is 10 meters, 5 meters on either side of the transect line (Continued)

\begin{tabular}{|c|c|c|c|}
\hline $\begin{array}{l}\text { Distance from } \\
\text { beginning of } \\
\text { transect, in } \mathrm{m}\end{array}$ & $\begin{array}{c}\text { Tree } \\
\text { species }\end{array}$ & Dbh, in $\mathbf{c m}$ & Side \\
\hline 58.3 & frapro & 25.0 & $\mathrm{e}$ \\
\hline 58.6 & frapro & 10.0 & $\mathrm{e}$ \\
\hline 59.0 & frapro & 15.2 & $\mathrm{w}$ \\
\hline 59.2 & nysbif & 27.0 & w \\
\hline 59.5 & snag & 37.8 & $\mathrm{e}$ \\
\hline 60.1 & frapro & 10.7 & $\mathrm{e}$ \\
\hline 61.0 & \multicolumn{3}{|c|}{ marker \# 162} \\
\hline 61.4 & myrcer & 11.0 & $\mathrm{e}$ \\
\hline \multirow{4}{*}{61.9} & \multirow{4}{*}{ magvir } & 56.5 & \multirow{4}{*}{$\mathrm{w}$} \\
\hline & & 18.0 & \\
\hline & & 13.5 & \\
\hline & & 12.3 & \\
\hline 64.0 & myrcer & 21.6 & $\mathrm{~W}$ \\
\hline 64.2 & myrcer & 16.0 & w \\
\hline 64.4 & myrcer & 10.5 & w \\
\hline 64.5 & ulmame & 24.9 & $\mathrm{e}$ \\
\hline 64.9 & sabpal & 18.7 & $\mathrm{e}$ \\
\hline 65.2 & sabpal & 22.4 & w \\
\hline 66.2 & quelau & 27.4 & $\mathrm{e}$ \\
\hline 66.5 & acerub & 12.7 & $\mathrm{e}$ \\
\hline 66.5 & sabpal & 24.7 & $\mathrm{e}$ \\
\hline 66.6 & acerub & 17.5 & $\mathrm{e}$ \\
\hline 66.6 & sabpal & 24.3 & $\mathrm{e}$ \\
\hline 67.8 & sabpal & 28.3 & w \\
\hline 69.5 & frapro & 12.4 & $\mathrm{e}$ \\
\hline 71.0 & frapro & 16.1 & $\mathrm{w}$ \\
\hline 71.5 & frapro & 10.9 & $\mathrm{e}$ \\
\hline 71.7 & frapro & 15.1 & $\mathrm{w}$ \\
\hline 72.2 & frapro & 16.6 & $\mathrm{w}$ \\
\hline 72.7 & nysbif & 10.9 & $\mathrm{w}$ \\
\hline 72.9 & frapro & 14.1 & $\mathrm{w}$ \\
\hline \multirow{3}{*}{73.9} & \multirow{3}{*}{ frapro } & 10.7 & \multirow{3}{*}{$\mathrm{e}$} \\
\hline & & 6.4 & \\
\hline & & 7.0 & \\
\hline 77.1 & nysbif & 48.4 & $\mathrm{w}$ \\
\hline 77.7 & frapro & 12.8 & $\mathrm{e}$ \\
\hline 78.0 & frapro & 19.6 & $\mathrm{e}$ \\
\hline 79.6 & magvir & 24.5 & $\mathrm{e}$ \\
\hline 81.1 & myrcer & 10.5 & $\mathrm{e}$ \\
\hline 81.6 & myrcer & 14.9 & w \\
\hline 81.6 & quelau & 40.0 & w \\
\hline 83.5 & sabpal & 23.9 & e \\
\hline 83.9 & sabpal & 25.5 & $\mathrm{w}$ \\
\hline 87.4 & sabpal & 30.4 & $\mathrm{e}$ \\
\hline 88.1 & myrcer & 20.1 & $\mathrm{w}$ \\
\hline 88.8 & myrcer & 10.0 & $\mathrm{w}$ \\
\hline
\end{tabular}

\begin{tabular}{|c|c|c|c|}
\hline $\begin{array}{l}\text { Distance from } \\
\text { beginning of } \\
\text { transect, in } \mathrm{m}\end{array}$ & $\begin{array}{c}\text { Tree } \\
\text { species }\end{array}$ & Dbh, in $\mathrm{cm}$ & Side \\
\hline 89.3 & sabpal & 28.0 & $\mathrm{~W}$ \\
\hline 89.5 & nysbif & 10.1 & $\mathrm{e}$ \\
\hline 90.5 & acerub & 11.2 & $\mathrm{w}$ \\
\hline \multirow{2}{*}{90.5} & \multirow{2}{*}{ magvir } & 18.3 & \multirow{2}{*}{$\mathrm{w}$} \\
\hline & & 8.5 & \\
\hline 91.0 & perpal & 15.3 & $\mathrm{w}$ \\
\hline 91.0 & sabpal & 25.2 & $\mathrm{w}$ \\
\hline 92.0 & sabpal & 18.0 & $\mathrm{w}$ \\
\hline 92.3 & frapro & 15.8 & $\mathrm{e}$ \\
\hline 93.5 & \multicolumn{3}{|c|}{ marker \# 163} \\
\hline 92.8 & frapro & 31.5 & $\mathrm{e}$ \\
\hline 94.3 & frapro & 17.4 & $\mathrm{e}$ \\
\hline 95.3 & frapro & 15.4 & $\mathrm{w}$ \\
\hline 96.2 & nysbif & 10.8 & $\mathrm{e}$ \\
\hline 96.9 & magvir & 11.6 & $\mathrm{e}$ \\
\hline 97.0 & magvir & 10.4 & $\mathrm{e}$ \\
\hline 97.5 & frapro & 11.8 & $\mathrm{e}$ \\
\hline 98.3 & frapro & 11.0 & $\mathrm{w}$ \\
\hline 98.8 & frapro & 14.4 & $\mathrm{e}$ \\
\hline 98.9 & magvir & 10.1 & $\mathrm{e}$ \\
\hline 99.8 & magvir & 12.6 & $\mathrm{e}$ \\
\hline \multirow{2}{*}{99.8} & \multirow{2}{*}{ magvir } & 23.3 & \multirow{2}{*}{$\mathrm{w}$} \\
\hline & & 13.5 & \\
\hline 100.1 & nysbif & 13.9 & $\mathrm{e}$ \\
\hline \multirow{3}{*}{101.0} & \multirow{3}{*}{ frapro } & 15.9 & \multirow{3}{*}{$\mathrm{e}$} \\
\hline & & 4.9 & \\
\hline & & 4.5 & \\
\hline 101.4 & magvir & 25.4 & $\mathrm{e}$ \\
\hline 102.3 & frapro & 10.9 & $\mathrm{e}$ \\
\hline 102.7 & magvir & 16.1 & $\mathrm{e}$ \\
\hline 104.9 & sabpal & 24.0 & $\mathrm{w}$ \\
\hline 105.2 & sabpal & 22.6 & $\mathrm{w}$ \\
\hline 105.4 & sabpal & 21.4 & $\mathrm{w}$ \\
\hline 107.3 & frapro & 22.4 & $\mathrm{e}$ \\
\hline 107.3 & frapro & 12.4 & $\mathrm{e}$ \\
\hline 107.8 & sabpal & 23.6 & w \\
\hline 110.6 & sabpal & 26.4 & w \\
\hline \multirow{2}{*}{111.2} & \multirow{2}{*}{ frapro } & 24.5 & \multirow{2}{*}{$\mathrm{e}$} \\
\hline & & 4.5 & \\
\hline 111.4 & ilecas & 13.7 & w \\
\hline 112.6 & frapro & 12.0 & e \\
\hline 113.6 & sabpal & 26.4 & w \\
\hline \multirow{2}{*}{113.7} & \multirow{2}{*}{ frapro } & 9.9 & \multirow{2}{*}{$\mathrm{e}$} \\
\hline & & 22.9 & \\
\hline 114.9 & frapro & 11.0 & $\mathrm{e}$ \\
\hline 115.1 & frapro & 10.5 & $\mathrm{e}$ \\
\hline
\end{tabular}


K LK: The sampling width of this transect is 10 meters, 5 meters on either side of the transect line (Continued)

\begin{tabular}{|c|c|c|c|}
\hline $\begin{array}{l}\text { Distance from } \\
\text { beginning of } \\
\text { transect, in } m\end{array}$ & $\begin{array}{c}\text { Tree } \\
\text { species }\end{array}$ & $\mathrm{Dbh}$, in $\mathrm{cm}$ & Side \\
\hline 115.9 & frapro & 15.3 & $\mathrm{e}$ \\
\hline 116.5 & frapro & 10.7 & w \\
\hline 116.6 & frapro & 9.2 & $\mathrm{e}$ \\
\hline 117.4 & \multicolumn{3}{|c|}{ marker \# 164} \\
\hline 118.6 & taxdis & 12.2 & $\mathrm{e}$ \\
\hline 119.3 & frapro & 11.4 & $\mathrm{w}$ \\
\hline 119.7 & nysbif & 10.4 & $\mathrm{e}$ \\
\hline \multirow{2}{*}{120.6} & \multirow{2}{*}{ nysbif } & 34.0 & \multirow{2}{*}{ e } \\
\hline & & 9.0 & \\
\hline 121.8 & nysbif & 11.7 & e \\
\hline 122.0 & nysbif & 14.6 & $\mathrm{w}$ \\
\hline 122.3 & frapro & 10.7 & $\mathrm{e}$ \\
\hline 122.4 & taxdis & 44.7 & $\mathrm{e}$ \\
\hline 123.4 & nysbif & 10.3 & $\mathrm{w}$ \\
\hline 124.8 & frapro & 10.2 & $\mathrm{e}$ \\
\hline 126.3 & nysbif & 13.5 & $\mathrm{e}$ \\
\hline 130.7 & taxdis & 21.2 & w \\
\hline 131.1 & taxdis & 14.9 & w \\
\hline 133.3 & taxdis & 19.4 & $\mathrm{w}$ \\
\hline 133.7 & taxdis & 24.3 & w \\
\hline 133.8 & taxdis & 23.2 & w \\
\hline 134.7 & taxdis & 34.6 & $\mathrm{e}$ \\
\hline 135.5 & frapro & 15.4 & $\mathrm{w}$ \\
\hline 139.8 & taxdis & 37.3 & w \\
\hline 142.1 & taxdis & 36.0 & w \\
\hline 143.2 & taxdis & 10.9 & $\mathrm{e}$ \\
\hline 145.5 & \multicolumn{3}{|c|}{ marker \# 165} \\
\hline
\end{tabular}


L DM: The sampling area for this transect is 10 meters, 5 meters either side of the transect line

\begin{tabular}{|c|c|c|c|}
\hline $\begin{array}{l}\text { Distance from } \\
\text { beginning of } \\
\text { transect, in } \mathrm{m}\end{array}$ & $\begin{array}{c}\text { Tree } \\
\text { species }\end{array}$ & Dbh, in $\mathrm{cm}$ & Side \\
\hline 0.0 & \multicolumn{3}{|c|}{ marker \# 166} \\
\hline 2.6 & nysbif & 31.9 & $\mathrm{w}$ \\
\hline 2.7 & nysbif & 28.0 & $\mathrm{w}$ \\
\hline 2.8 & taxdis & 45.7 & $\mathrm{w}$ \\
\hline 3.4 & frapro & 11.6 & $\mathrm{e}$ \\
\hline 4.5 & frapro & 13.6 & $\mathrm{w}$ \\
\hline 4.6 & nysbif & 22.1 & e \\
\hline 4.7 & magvir & 13.3 & $\mathrm{e}$ \\
\hline 4.8 & frapro & 10.3 & $\mathrm{e}$ \\
\hline 5.3 & nysbif & 31.8 & $\mathrm{e}$ \\
\hline 7.9 & nysbif & 13.0 & $\mathrm{w}$ \\
\hline 8.5 & snag & 11.5 & $\mathrm{e}$ \\
\hline 8.8 & nysbif & 23.4 & $\mathrm{w}$ \\
\hline 8.9 & nysbif & 24.5 & $\mathrm{e}$ \\
\hline 10.4 & nysbif & 28.2 & $\mathrm{w}$ \\
\hline 10.6 & magvir & 19.7 & $\mathrm{e}$ \\
\hline 10.7 & frapro & 29.6 & $\mathrm{e}$ \\
\hline 11.0 & magvir & 18.2 & $\mathrm{e}$ \\
\hline 11.4 & taxdis & 18.6 & $\mathrm{w}$ \\
\hline 11.5 & taxdis & 14.0 & $\mathrm{w}$ \\
\hline 11.6 & magvir & 13.1 & $\mathrm{e}$ \\
\hline 12.1 & magvir & 18.8 & e \\
\hline 13.1 & frapro & 18.0 & $\mathrm{e}$ \\
\hline 13.1 & frapro & 8.3 & $\mathrm{e}$ \\
\hline 13.1 & snag & 15.4 & $\mathrm{w}$ \\
\hline 13.3 & \multicolumn{3}{|c|}{ marker \# 167} \\
\hline 14.8 & frapro & 11.0 & $\mathrm{w}$ \\
\hline 15.2 & magvir & 12.3 & $\mathrm{e}$ \\
\hline 16.2 & nysbif & 14.0 & $\mathrm{e}$ \\
\hline 17.0 & frapro & 11.1 & $\mathrm{e}$ \\
\hline 17.1 & nysbif & 14.7 & $\mathrm{e}$ \\
\hline 17.4 & frapro & 13.0 & $\mathrm{~W}$ \\
\hline 17.5 & nysbif & 38.3 & $\mathrm{~W}$ \\
\hline 18.6 & nysbif & 29.8 & e \\
\hline 19.9 & frapro & 14.4 & w \\
\hline 20.7 & frapro & 12.1 & $\mathrm{e}$ \\
\hline 20.8 & nysbif & 34.2 & $\mathrm{w}$ \\
\hline 21.2 & nysbif & 29.4 & $\mathrm{e}$ \\
\hline 21.4 & frapro & 12.2 & $\mathrm{w}$ \\
\hline 22.1 & nysbif & 27.7 & $\mathrm{e}$ \\
\hline 22.7 & frapro & 10.8 & w \\
\hline 22.7 & frapro & 4.9 & W \\
\hline 23.8 & frapro & 12.5 & $\mathrm{~W}$ \\
\hline 24.0 & frapro & 13.2 & $\mathrm{e}$ \\
\hline 26.4 & nysbif & 31.4 & $\mathrm{e}$ \\
\hline 26.8 & frapro & 11.0 & $\mathrm{w}$ \\
\hline 26.8 & nysbif & 27.5 & W \\
\hline 26.8 & nysbif & 31.1 & $\mathrm{w}$ \\
\hline \multirow{2}{*}{26.9} & \multirow{2}{*}{ nysbif } & 23.0 & \multirow{2}{*}{ e } \\
\hline & & 7.7 & \\
\hline
\end{tabular}

\begin{tabular}{|c|c|c|c|}
\hline $\begin{array}{l}\text { Distance from } \\
\text { beginning of } \\
\text { transect, in } \mathrm{m}\end{array}$ & $\begin{array}{c}\text { Tree } \\
\text { species }\end{array}$ & Dbh, in $\mathrm{cm}$ & Side \\
\hline 28.5 & frapro & 13.7 & $\mathrm{e}$ \\
\hline 29.5 & nysbif & 39.4 & $\mathrm{e}$ \\
\hline 29.9 & nysbif & 15.2 & w \\
\hline 30.4 & nysbif & 25.2 & $\mathrm{w}$ \\
\hline 31.3 & \multicolumn{3}{|c|}{ marker \# 168} \\
\hline 31.6 & nysbif & 15.9 & w \\
\hline 32.2 & nysbif & 26.3 & $\mathrm{e}$ \\
\hline 32.8 & nysbif & 42.4 & $\mathrm{w}$ \\
\hline 33.8 & frapro & 10.5 & $\mathrm{w}$ \\
\hline 33.9 & magvir & 11.8 & $\mathrm{e}$ \\
\hline 35.0 & nysbif & 40.2 & $\mathrm{w}$ \\
\hline 36.4 & frapro & 12.6 & $\mathrm{w}$ \\
\hline 36.4 & frapro & 7.6 & w \\
\hline 37.0 & nysbif & 40.0 & $\mathrm{e}$ \\
\hline 37.0 & taxdis & 45.7 & $\mathrm{e}$ \\
\hline 37.1 & frapro & 18.5 & $\mathrm{w}$ \\
\hline 39.6 & frapro & 12.0 & $\mathrm{e}$ \\
\hline 39.6 & frapro & 12.1 & $\mathrm{w}$ \\
\hline 40.3 & frapro & 14.3 & $\mathrm{e}$ \\
\hline 41.2 & magvir & 12.5 & $\mathrm{w}$ \\
\hline 42.1 & frapro & 10.3 & $\mathrm{e}$ \\
\hline 42.6 & frapro & 19.0 & $\mathrm{w}$ \\
\hline 43.5 & frapro & 11.6 & $\mathrm{e}$ \\
\hline \multirow{2}{*}{43.8} & \multirow{2}{*}{ frapro } & 10.1 & \multirow{2}{*}{ e } \\
\hline & & 13.0 & \\
\hline 43.9 & frapro & 14.4 & $\mathrm{w}$ \\
\hline 44.1 & magvir & 11.4 & $\mathrm{w}$ \\
\hline 44.3 & frapro & 12.5 & $\mathrm{w}$ \\
\hline 45.1 & frapro & 11.6 & $\mathrm{w}$ \\
\hline 45.5 & frapro & 10.8 & $\mathrm{e}$ \\
\hline 45.9 & frapro & 11.6 & $\mathrm{w}$ \\
\hline 46.0 & frapro & 11.6 & $\mathrm{e}$ \\
\hline \multirow{2}{*}{46.6} & \multirow{2}{*}{ frapro } & 10.4 & \multirow{2}{*}{ e } \\
\hline & & 8.2 & \\
\hline 47.7 & frapro & 15.0 & $\mathrm{e}$ \\
\hline 47.7 & frapro & 14.2 & $\mathrm{w}$ \\
\hline 48.3 & frapro & 15.4 & $\mathrm{w}$ \\
\hline 48.6 & frapro & 12.7 & $\mathrm{w}$ \\
\hline 48.8 & frapro & 11.9 & $\mathrm{e}$ \\
\hline 49.5 & magvir & 13.8 & $\mathrm{w}$ \\
\hline 49.8 & frapro & 22.2 & $\mathrm{w}$ \\
\hline 51.0 & magvir & 10.2 & $\mathrm{w}$ \\
\hline 51.4 & nysbif & 14.7 & $\mathrm{e}$ \\
\hline 51.4 & nysbif & 15.9 & $\mathrm{w}$ \\
\hline 51.7 & frapro & 18.4 & $\mathrm{~W}$ \\
\hline 52.8 & frapro & 10.1 & $\mathrm{~W}$ \\
\hline 53.1 & nysbif & 49.4 & $\mathrm{e}$ \\
\hline 53.2 & \multicolumn{3}{|c|}{ marker \# 169} \\
\hline
\end{tabular}


Appendix 2. Conversion tables for determining the permanent marker numbers of all previous stake numbers recorded in original U.S. Geological Survey field notes

\begin{tabular}{|c|c|}
\hline \multicolumn{2}{|c|}{ CONFLUENCE } \\
\hline $\begin{array}{l}\text { Stake number } \\
\text { in original } \\
\text { field notes }\end{array}$ & $\begin{array}{c}\text { Permanent } \\
\text { marker } \\
\text { number }\end{array}$ \\
\hline \multicolumn{2}{|c|}{ West segment } \\
\hline 1 & 1 \\
\hline 2 & 2 \\
\hline 3 & 3 \\
\hline 4 & 4 \\
\hline 5 & 5 \\
\hline 6 & 6 \\
\hline 7 & 7 \\
\hline \multicolumn{2}{|c|}{ East segment } \\
\hline 1 & 8 \\
\hline 2 & 9 \\
\hline 3 & 10 \\
\hline 4 & 11 \\
\hline 5 & 12 \\
\hline 6 & 13 \\
\hline 7 & 14 \\
\hline 8 & 15 \\
\hline 9 & 16 \\
\hline 10 & 17 \\
\hline 11 & 18 \\
\hline 12 & 19 \\
\hline 13 & 20 \\
\hline 14 & 21 \\
\hline
\end{tabular}

\begin{tabular}{|c|c|}
\hline \multicolumn{2}{|c|}{ LOG LANDING } \\
\hline $\begin{array}{l}\text { Stake number } \\
\text { in original } \\
\text { field notes }\end{array}$ & $\begin{array}{c}\text { Permanent } \\
\text { marker } \\
\text { number }\end{array}$ \\
\hline 1 & 22 \\
\hline 2 & 23 \\
\hline 3 & 24 \\
\hline 4 & 25 \\
\hline 5 & 26 \\
\hline 6 & 27 \\
\hline 7 & 28 \\
\hline 8 & 29 \\
\hline 9 & 30 \\
\hline 10 & 31 \\
\hline 11 & 32 \\
\hline 12 & 33 \\
\hline 13 & 34 \\
\hline 14 & 35 \\
\hline 15 & 36 \\
\hline 16 & 37 \\
\hline 17 & 38 \\
\hline $18 \mathrm{a}$ & 39 \\
\hline $18 \mathrm{~b}$ & 40 \\
\hline 19 & 41 \\
\hline 20 & 42 \\
\hline 21 & 43 \\
\hline 22 & 44 \\
\hline 23 & 45 \\
\hline 24 & 46 \\
\hline 25 & 47 \\
\hline 26 & 48 \\
\hline 27 & 49 \\
\hline 28 & 50 \\
\hline 29 & 51 \\
\hline
\end{tabular}

FALKENBURRY

\begin{tabular}{|c|c|}
\hline $\begin{array}{l}\text { Stake number } \\
\text { in original } \\
\text { field notes }\end{array}$ & $\begin{array}{c}\text { Permanent } \\
\text { marker } \\
\text { number }\end{array}$ \\
\hline \multicolumn{2}{|c|}{ East segment } \\
\hline 1 & 52 \\
\hline 2 & 53 \\
\hline 3 & 54 \\
\hline 4 & 55 \\
\hline 5 & 56 \\
\hline 6 & 57 \\
\hline 7 & 58 \\
\hline \multicolumn{2}{|c|}{ West segment } \\
\hline 8 & 59 \\
\hline 7 & 60 \\
\hline 6 & 61 \\
\hline 5 & 62 \\
\hline 4 & 63 \\
\hline 3 & 64 \\
\hline 2 & 65 \\
\hline 1 & 66 \\
\hline
\end{tabular}


Appendix 2. Conversion tables for determining the permanent marker numbers of all previous stake numbers recorded in original U.S. Geological Survey field notes (Continued)

\section{MANATEE SPRINGS}

\begin{tabular}{|c|c|}
\hline $\begin{array}{l}\text { Stake number } \\
\text { in original } \\
\text { field notes }\end{array}$ & $\begin{array}{l}\text { Permanent } \\
\text { marker } \\
\text { number }\end{array}$ \\
\hline 1 new & 67 \\
\hline 2 new & 68 \\
\hline 3 new & 69 \\
\hline 4 new & 70 \\
\hline 5 new & 71 \\
\hline 6 new & 72 \\
\hline 7 new & 73 \\
\hline 8 new & 74 \\
\hline 9 new & 75 \\
\hline 10 new & 76 \\
\hline 11 new & 77 \\
\hline 12 & 78 \\
\hline 13 & 79 \\
\hline 14 & 80 \\
\hline 16 & 81 \\
\hline 17 & 82 \\
\hline 11 & 83 \\
\hline 10 & 84 \\
\hline 9 & 85 \\
\hline 8 & 86 \\
\hline 7 & 87 \\
\hline 6 & 88 \\
\hline 5 & 89 \\
\hline 4 & 90 \\
\hline 3 & 91 \\
\hline 2 & 92 \\
\hline 1 & 93 \\
\hline 0 & 94 \\
\hline
\end{tabular}

\section{KEEN}

\begin{tabular}{|c|c|}
\hline $\begin{array}{l}\text { Stake number } \\
\text { in original } \\
\text { field notes }\end{array}$ & $\begin{array}{l}\text { Permanent } \\
\text { marker } \\
\text { number }\end{array}$ \\
\hline 1 & 95 \\
\hline 2 & 96 \\
\hline 3 & 97 \\
\hline 4 & 98 \\
\hline 5 & 99 \\
\hline 6 & 100 \\
\hline 7 & 101 \\
\hline 8 & 102 \\
\hline 9 & 103 \\
\hline 10 & 104 \\
\hline 11 & 105 \\
\hline 12 & 106 \\
\hline 13 & 107 \\
\hline 14 & 108 \\
\hline 15 & 109 \\
\hline 16 & 110 \\
\hline 17 & 111 \\
\hline 18 & 112 \\
\hline 19 & 113 \\
\hline 20 & 114 \\
\hline 21 & 115 \\
\hline 22 & 116 \\
\hline 23 & 117 \\
\hline 24 & 118 \\
\hline 25 & 119 \\
\hline 26 & 120 \\
\hline 27 & 121 \\
\hline
\end{tabular}

\section{KEEN ISLAND}

\begin{tabular}{|c|c|}
\hline $\begin{array}{c}\text { Stake number } \\
\text { in original field } \\
\text { notes }\end{array}$ & $\begin{array}{c}\text { Permanent } \\
\text { marker number }\end{array}$ \\
\hline A & 122 \\
\hline B & 123 \\
\hline C & 124 \\
\hline D & 125 \\
\hline
\end{tabular}

\section{TURKEY ISLAND}

\begin{tabular}{|c|c|}
\hline $\begin{array}{c}\text { Stake number } \\
\text { in original } \\
\text { field notes }\end{array}$ & $\begin{array}{c}\text { Permanent } \\
\text { marker } \\
\text { number }\end{array}$ \\
\hline 1 & 126 \\
\hline 2 & 127 \\
\hline 3 & 128 \\
\hline 4 & 129 \\
\hline 5 & 130 \\
\hline 6 & 131 \\
\hline 7 & 132 \\
\hline 8 & 133 \\
\hline 9 & 134 \\
\hline 10 & 135 \\
\hline 11 & 136 \\
\hline 12 & 137 \\
\hline 13 & 138 \\
\hline 14 & 139 \\
\hline 15 & 140 \\
\hline
\end{tabular}


Appendix 2. Conversion tables for determining the permanent marker numbers of all previous stake numbers recorded in original U.S. Geological Survey field notes (Continued)

SANDFLY NORTH

\begin{tabular}{|c|c|}
\hline $\begin{array}{c}\text { Stake number } \\
\text { in original } \\
\text { field notes }\end{array}$ & $\begin{array}{c}\text { Permanent } \\
\text { marker } \\
\text { number }\end{array}$ \\
\hline 1 & 141 \\
\hline 2 & 142 \\
\hline 3 & 143 \\
\hline 4 & 144 \\
\hline 5 & 145 \\
\hline
\end{tabular}

LOCK CREEK

\begin{tabular}{|c|c|}
\hline $\begin{array}{c}\text { Stake number } \\
\text { in original } \\
\text { field notes }\end{array}$ & $\begin{array}{c}\text { Permanent } \\
\text { marker } \\
\text { number }\end{array}$ \\
\hline 1 & 160 \\
\hline 2 & 161 \\
\hline 3 & 162 \\
\hline 4 & 163 \\
\hline 5 & 164 \\
\hline 6 & 165 \\
\hline
\end{tabular}

SANDFLY HAMMOCK

\begin{tabular}{|c|c|}
\hline $\begin{array}{c}\text { Stake num- } \\
\text { ber in original } \\
\text { field notes }\end{array}$ & $\begin{array}{c}\text { Permanent } \\
\text { marker } \\
\text { number }\end{array}$ \\
\hline 1 & 146 \\
\hline 2 & 147 \\
\hline 3 & 148 \\
\hline 4 & 149 \\
\hline 5 & 150 \\
\hline 6 & 151 \\
\hline
\end{tabular}

\section{DEMORY CREEK}

\begin{tabular}{|c|c|}
\hline $\begin{array}{c}\text { Stake num- } \\
\text { ber in original } \\
\text { field notes }\end{array}$ & $\begin{array}{c}\text { Permanent } \\
\text { marker } \\
\text { number }\end{array}$ \\
\hline 1 & 166 \\
\hline 2 & 167 \\
\hline 3 & 168 \\
\hline 4 & 169 \\
\hline
\end{tabular}

BARNETT CREEK

\begin{tabular}{|c|c|}
\hline $\begin{array}{c}\text { Stake number } \\
\text { in original } \\
\text { field notes }\end{array}$ & $\begin{array}{c}\text { Permanent } \\
\text { marker } \\
\text { number }\end{array}$ \\
\hline 1 & 152 \\
\hline 2 & 153 \\
\hline 3 & 154 \\
\hline 4 & 155 \\
\hline 5 & 156 \\
\hline 6 & 157 \\
\hline 7 & 158 \\
\hline 8 & 159 \\
\hline
\end{tabular}

